Florida International University FIU Digital Commons

$5-25-2018$

\title{
Antibiotic Resistance and Cell-Wall Recycling in Pseudomonas aeruginosa
}

Supurna Dhar

sdhar003@fiu.edu

DOI: $10.25148 /$ etd.FIDC006816

Follow this and additional works at: https://digitalcommons.fiu.edu/etd

Part of the Medicine and Health Sciences Commons

\section{Recommended Citation}

Dhar, Supurna, "Antibiotic Resistance and Cell-Wall Recycling in Pseudomonas aeruginosa" (2018). FIU Electronic Theses and Dissertations. 3817.

https://digitalcommons.fiu.edu/etd/3817

This work is brought to you for free and open access by the University Graduate School at FIU Digital Commons. It has been accepted for inclusion in FIU Electronic Theses and Dissertations by an authorized administrator of FIU Digital Commons. For more information, please contact dcc@fiu.edu. 


\title{
FLORIDA INTERNATIONAL UNIVERSITY
}

Miami, Florida

\section{ANTIBIOTIC RESISTANCE AND CELL-WALL RECYCLING IN PSEUDOMONAS AERUGINOSA}

\author{
A dissertation submitted in partial fulfillment of \\ the requirements for the degree of \\ DOCTOR OF PHILOSOPHY \\ in \\ BIOMEDICAL SCIENCES \\ by \\ Supurna Dhar
}

2018 
To: Dean John Rock

College of Medicine

This dissertation, written by Supurna Dhar, and entitled and Antibiotic Resistance and Cell-Wall Recycling in Pseudomonas aeruginosa, having been approved in respect to style and intellectual content, is referred to you for judgment.

We have read this dissertation and recommend that it be approved.

$\begin{array}{r}\hline \text { Jeremy Chambers } \\ \hline \text { Yukching Tse-Dinh } \\ \hline \text { Marisela Agudelo } \\ \hline \text { Shahriar Mobashery } \\ \hline \text { Kalai Mathee, Major Professor }\end{array}$

Date of Defense: May 25, 2018

The dissertation of Supurna Dhar is approved.

Dean John Rock College of Medicine

Andrés G. Gil Vice President for Research and Economic Development and Dean of the University Graduate School

Florida International University, 2018 


\section{ACKNOWLEDGMENTS}

Firstly, I would like to acknowledge my mentor Dr. Kalai Mathee for all her guidance, support and encouragement over the years. Her intellectual curiosity, enthusiasm and dedication are an inspiration. I sincerely acknowledge all my dissertation committee members Dr. Jeremy Chambers, Dr. Marisela Agudelo, Dr. Yuk ching Tse-Dinh and Dr. Shahriar Mobashery for their critical suggestions, and helpful discussions on my research. I am extremely grateful to Dr. Mobashery for allowing me to spend summer of 2014 and 2017 in his lab. It was an excellent opportunity to conduct important research and interact with his talented lab members who were all extremely welcoming! I am very grateful to Dr. Mijoon Lee for her remarkable guidance on my project. Thanks to Dr. Gagani Athauda and Dr. Weiler as my mentors during my TA. I would also like to acknowledge Dr. Agoulnik, our Program Director for his constant support throughout the Ph.D. program. I am also very grateful for the support provided by Herbert Wertheim College of Medicine in form of Teaching and Graduate assistantships. I am thankful for the funding provided by National Institutes of Health-MBRS RISE program for a summer research award, 2017 and to University Graduate School, FIU for the Dissertation Year Fellowship.

Ph.D. has its own highs and lows and I am glad I had a group of amazing friends with me on this journey. My friends from Manipal- Gayathri, Gowri, Forum and Mommy Kumari. Cheryl Engineer, for becoming my extended family here. My friends at FIU Adwait, Mansi, Priyanka, Ketaki, Mayur, Sowmya, and KC. Shashank, Iru, and Jiao JiaoI joined FIU with them and I couldn't have asked for a better cohort. I am thankful to have spent these years with the members of the Mathee lab, an extraordinary and purposeful group of people, both past and present-Deepak, Diansy, Gorakh, Sundar, 
Jonathon, Kyle, Kevin, Sameer, Brett, MariaVictoria, and Ana. I am grateful to Dr. Hansi Kumari for taking me under her wing and providing me with outstanding mentoring. Finally, my family, especially my parents- Mr. Suman and Mrs. Sanchita Dhar, and my brother Dr. Sujan Dhar and sis in-law Ms Deepti Nair for providing support, love and good cheer always. 


\section{ABSTRACT OF THE DISSERTATION}

\section{ANTIBIOTIC RESISTANCE AND CELL-WALL RECYCLING IN PSEUDOMONAS}

\section{AERUGINOSA}

by

\section{Supurna Dhar}

Florida International University, 2018

Miami, Florida

\section{Professor Kalai Mathee, Major Professor}

An exponential increase in infections by multi-drug, extensive-drug, and pan-drug resistant bacteria in both developed and developing countries are poised to return us to the pre-antibiotic era. Pseudomonas aeruginosa, a Gram-negative bacterium is frequently associated with fatal infections and has been classified by the WHO and the CDC as a pathogen of "critical" importance, and serious threat, respectively. $P$. aeruginosa infections are often treated using a combination of a B-lactam and an aminoglycoside. Resistance to ß-lactams is conferred by the constitutive expression of a chromosomally-encoded AmpC B-lactamase. The ampC expression is regulated by $A m p R$, a membrane-spanning transcriptional regulator of the LysR family. AmpR is presumed to be activated by muramyl peptides generated in the presence of B-lactams. The muramyl peptides are assumed to shuttle using two permeases, PA4393 (AmpG) and PA4218 (AmpP), importing and exporting into and out of periplasm from the cytoplasm, respectively. To date, the identity of $P$. aeruginosa muramyl peptides, and the AmpR effectors, the AmpG and AmpP substrates have not been elucidated. Using liquid chromatography/mass spectrometry analyses, we clarified the structures and quantified the muramyl peptides of wild-type $P$. aeruginosa PAO1. Twenty muropeptides from PAO1 were identified; and the least and the most metabolites are 100 and 55,000 
molecules per bacterium, respectively. Two AmpR-signaling muropeptides, Nacetylglucosamine-1,6-anhydro-N-acetylmuramyl pentapeptide and 1,6-anhydro-Nacetylmuramyl pentapeptide were identified. The role of two permeases in antibiotic resistance and cell-wall recycling were investigated by comparing PAO1 with its isogenic $\Delta a m p G, \Delta a m p P$, and $\triangle a m p G a m p P$ mutants. Our study demonstrates that AmpG and AmpP may play a role in transport of disaccharide-muropeptides. The muropeptide profiles alluded to the presence of a novel periplasmic $\mathrm{N}$-acetyl $\beta-\mathrm{D}$ glucosaminidase and led to the analyses of PA1085 (FlgJ). The loss of flgJ resulted in affecting swimming and biofilm production but failed to abolish the periplasmic glucosaminidase activity. In our effort to identify inhibitors of $\mathrm{AmpR}$, novel reporter-based screening assays were developed using AmpR-regulated $\mathrm{P}_{\text {ampc }}, \mathrm{P}_{\text {phzA1 }}$, and $\mathrm{P}_{\operatorname{mex}}$ promoters. We have identified pyrollidine-based scaffold library with the highest inhibitory effect against the AmpR system. In summary, this dissertation increases our understanding of cell-wall recycling and mechanisms of $\beta$-lactam resistance and attempts at establishing novel-antibacterial targets. 


\section{TABLE OF CONTENTS}

CHAPTER $\quad$ PAGE

\section{CHAPTER 1}

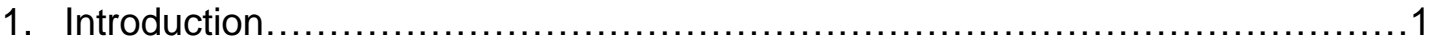

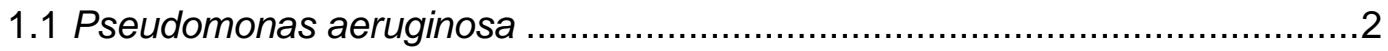

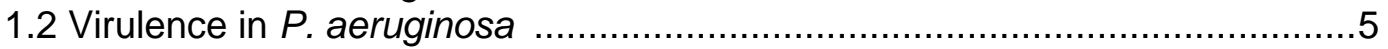

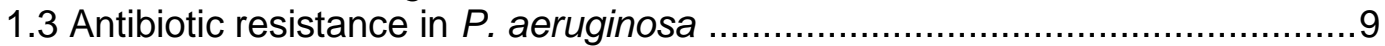

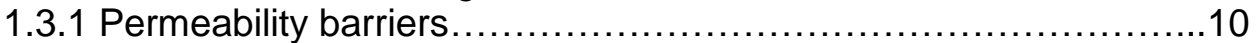

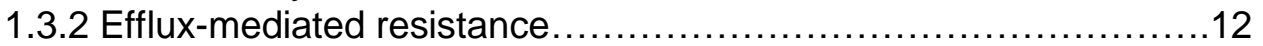

1.3.3 Target modification ............................................................. 13

1.3.4 $\beta$-lactamase mediated resistance ........................................... 14

1.4 Regulation of AmpC $\beta$-lactamase expression......................................... 18

1.5 Cell-wall recycling and synthesis in Escherichia coli and $P$. aeruginosa.......18

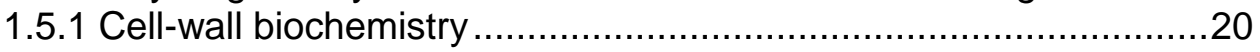

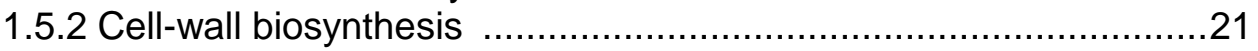

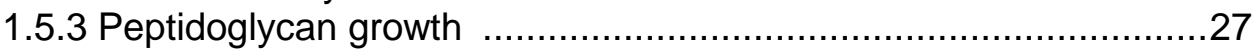

1.5.4 Cell-wall recycling ............................................................ 28

1.5.5 Convergence of recycling and biosynthesis ............................. 42

1.6. Cell-wall recycling and antibiotic resistance ........................................ 44

1.6.1 $\beta$-Lactam resistance involving Amp pathway ...........................45

1.6.2 $\beta$-Lactam resistance through other cell-wall-related genes ...........50

1.6.3 Indirect regulation of $\beta$-lactam resistance through AmpR ..............52

1.6.4 Cell-wall components contributing to non- $\beta$-lactam resistance ......53

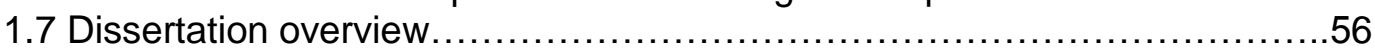

Chapter 2

2. Muropeptides in Pseudomonas aeruginosa and their role as elicitors of

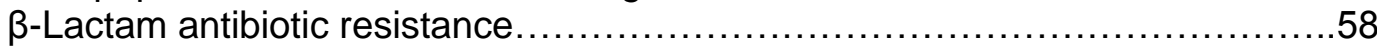

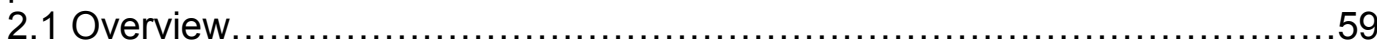

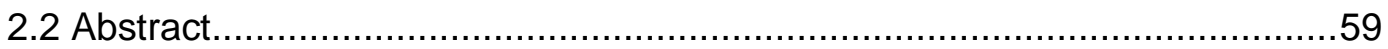

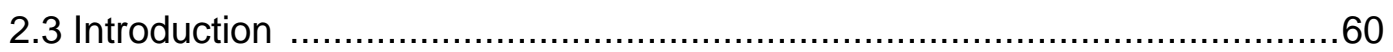

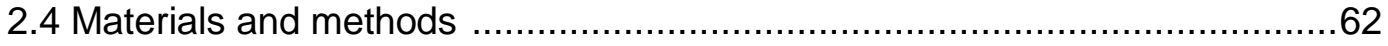

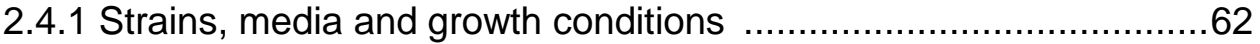

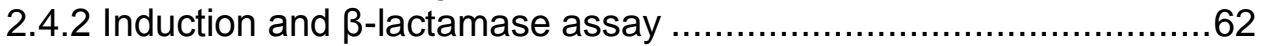

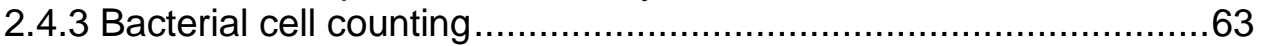

2.4.4 Syntheses of authentic muropeptides $\quad$......................63

2.4.5 Sample preparation: release of muramyl peptides from

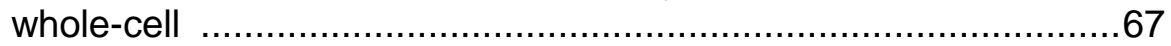

2.4.6 Detection and quantification of muropeptides by LC/MS .............67

2.4.7 Calculation of number of muropeptides per cell ...........................68

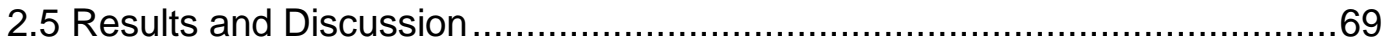

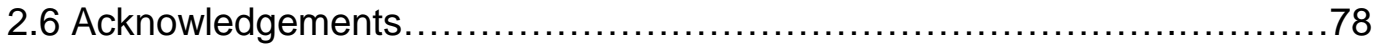

Chapter 3

3. Role of $P$. aeruginosa permeases PA4393 and PA4218 in antibiotic resistance and cell-wall recycling.... 


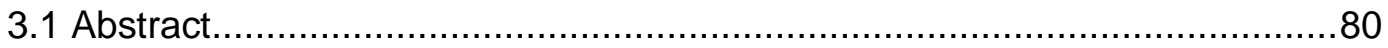

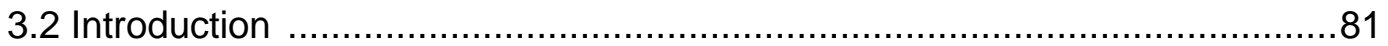

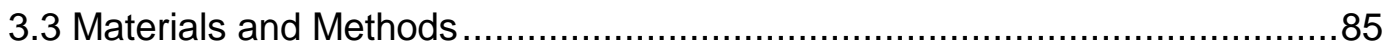

3.3.1 Strains, media and growth conditions ………………………..... 85

3.3.2 Construction of ampG and ampP deletion mutants by homologous

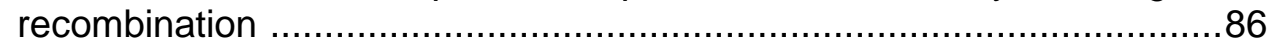

3.3.3 Construction of complementing plasmids....................................89

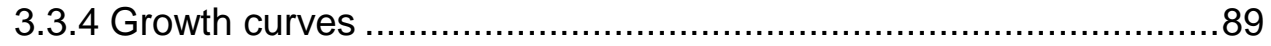

3.3.5 Determination of minimum inhibitory concentration (MIC) …..........90

3.3.6 Quantifying $\beta$-lactamase activity ...............................................90

3.3.7 Release of muramyl peptides from whole cell extracts ..................90

3.3.8 Release of muramyl peptides from periplasm …….....................91

3.3.9 Detection of muropeptides by liquid chromatography/mass

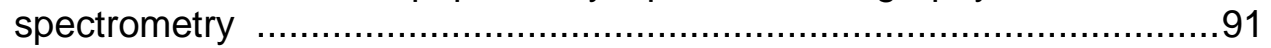

3. 3.10 Statistical analyses …………...........................................92

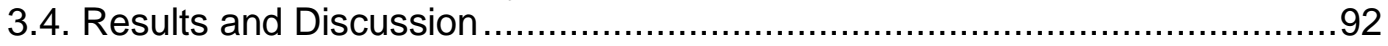

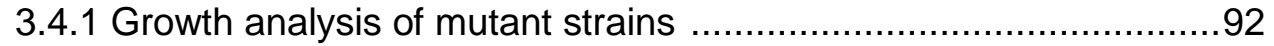

3.4.2 MIC of $\beta$-lactams is altered in ampG deletion .............................93

3.4.3 $\beta$-lactamase expression is reduced in ampG deletion mutants .....94

3.4.4 MIC and $\beta$-lactamase expression is unaltered in ampP deletion ...96

3.4.5 Muropeptide profile of whole-cell extract in mutant strains .............98

3.4.6 Muropeptide profile of periplasmic extract in mutant strains ..........99

3.4.7 Decrease of disaccharide in PAO $\triangle a m p P$ periplasm and increase presence of disaccharide in PAO $\mathrm{PampG}$ periplasm...........................102

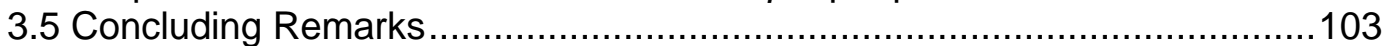

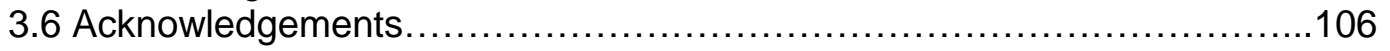

Chapter 4

4. Elucidating the role of PA1085 in Pseudomonas aeruginosa........................107

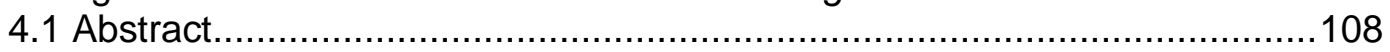

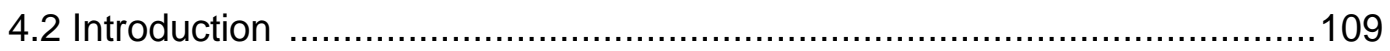

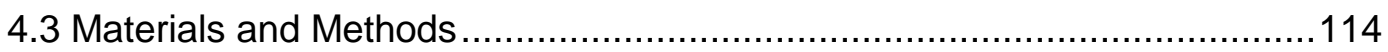

4.3.1 Strains, media and growth conditions ……………………......114

4.3.2 Construction of flgJ deletion mutants by homologous recombination using Gibson cloning …………………..........115

4.3.3 Construction of flg $J$ complementing clones................................116

4.3.4 Minimum-inhibitory concentration (MIC) ………………….....117

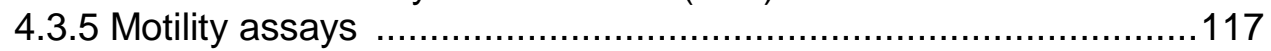

4.3.6 Biofilm assay .................................................................117

4.3.7 N-acetylglucosaminidase assay ..............................................118

4.4 Results .......................................................................................118

4.4.1 flg $\mathrm{J}$ is required for swimming motility .....................................118

4.4.2 flg $\mathrm{J}$ is required for initiation of biofilm .....................................119

4.4.3 MIC of selected antibiotics is unaltered in absence of flgJ ..........120

4.4.4 N-acetylglucosaminidase assay ............................................121

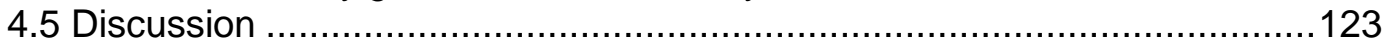


Chapter 5

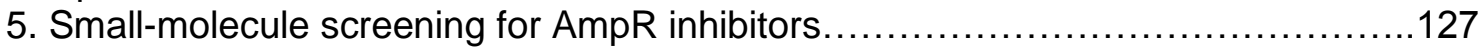

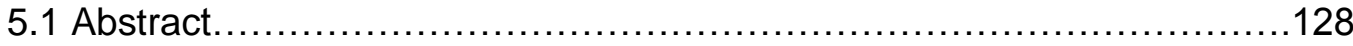

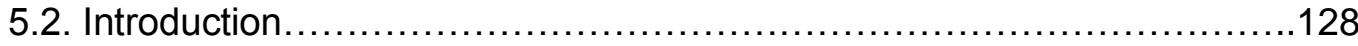

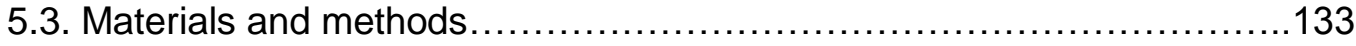

5.3.1. Strains, media and growth conditions.........................133

5.3.2. Membrane fractionation..........................................

5.3.3. Modeling of AmpR...........................................134

5.3.4. Reporter construction for luciferase assays....................134

5.3.5 Luciferase assays of reporter strains...........................138

5.3.6 Primary screen with compounds from Torrey Pines...............138

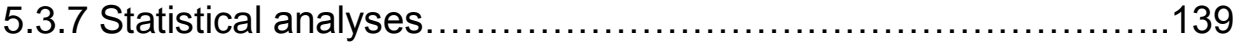

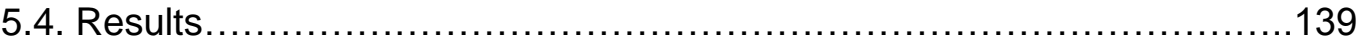

5.4.1. AmpR is localized in the membrane............................139

5.4.2. Gly102Glu leads to structural instability whereas Asp135Asn stabilizes AmpR dimer..............................................................140

5.4 .3 Validation of reporter constructs............................. 142

5.4.4. Primary screen of Torrey Pines Compounds......................144

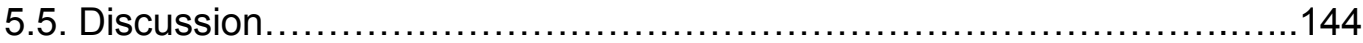

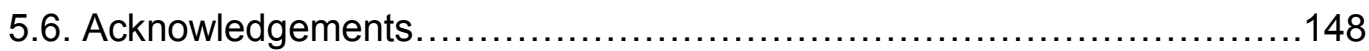

Chapter 6

6. Overview, Summary and Future directions..................................... 149

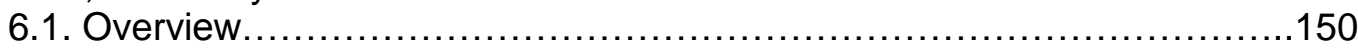

6. 2. Muropeptides in $P$. aeruginosa and AmpR effector.........................153

6. 3. AmpG and AmpP play a role in cell wall recycling..........................156

6. 4. Role of PA1085 in P. aeruginosa.......................................157

6.5. Screening of small molecule library for AmpR inhibitors ...................158

6.6. Future Directions .................................................. 159

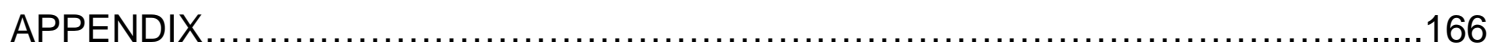

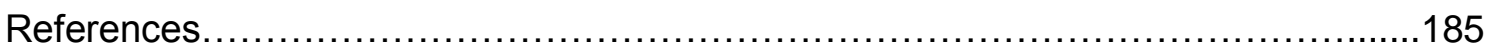

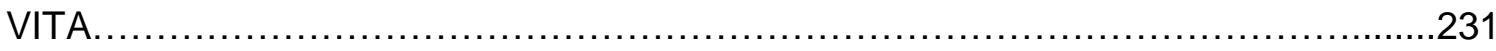




\section{LIST OF TABLES}

TABLE

PAGE

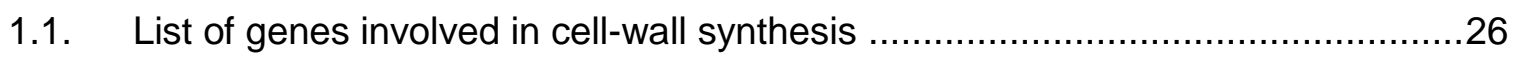

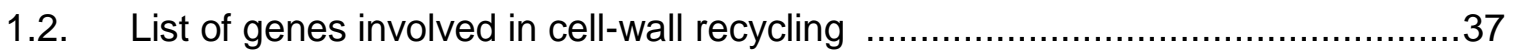

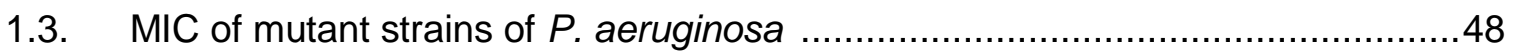

2.1. Detected muropeptides from whole-cell analysis ...................................... 74

2.2. Induction of $\beta$-lactamase activity by three inducers .....................................77

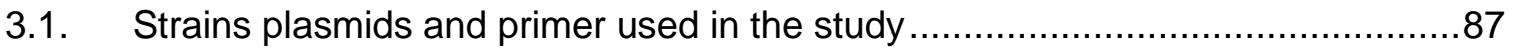

3. 2. Susceptibility profile of $P A O \Delta a m p G, P A O \triangle a m p P$ and $P A O \Delta a m p G \Delta a m p P \ldots . .95$

4.1. $\quad$ Protein homology between $S$. enterica and $P$. aeruginosa FlgJ .....................111

4.2. Strains, plasmid and primer used in this study .......................................114

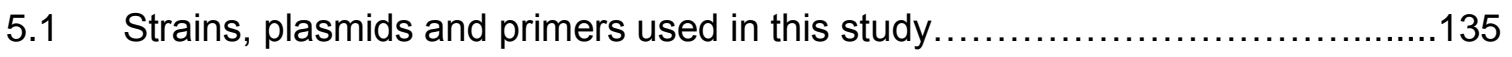




\section{LIST OF FIGURES}

FIGURE

PAGE

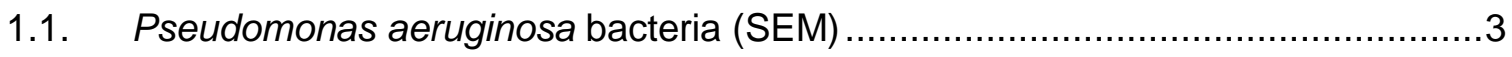

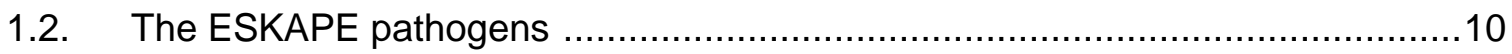

1.3. Peptidoglycan recycling and synthesis pathway in E. coli. ...........................21

1.4. $\quad$ Outline of cell-wall recycling in $P$. aeruginosa. ..........................................

1.5. Small molecule inhibitors of $\mathrm{N}$-acetyl- $\beta$-glucosaminidase NagZ .......................48

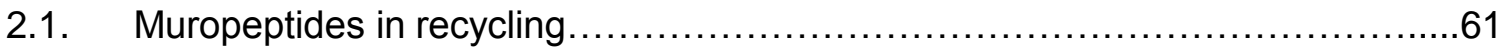

2.2. The chemical structures of authentic muropeptides used in this study...............65

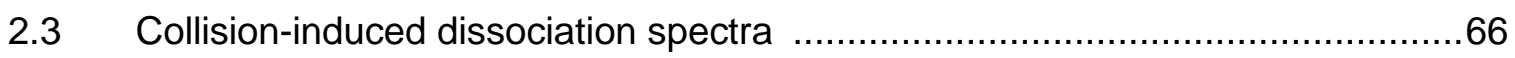

2.4 Standard curves of synthetic standards, $2 \mathrm{e}, 4 \mathrm{c}, 4 \mathrm{~d}, 4 \mathrm{e}$, and $7 \ldots \ldots \ldots \ldots \ldots \ldots \ldots$

2.5. A Chemical structures of detected muropeptides .........................................70

2.5.B The chemoenzymatic synthesis of six muropeptides …..............................71

2.6. Analysis of $P$. aeruginosa muropeptides................................... 73

2.7. Alignment of PA1085 (PA) and FlgJ (SE) sequence................................75

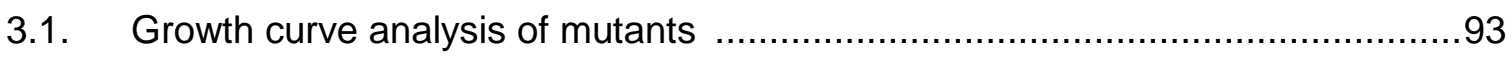

3.2. $\quad \beta$-lactamase expression of PAOI and permease mutants. .............................96

3.3.A A brief scheme of the muropeptide extraction protocol. ..............................97

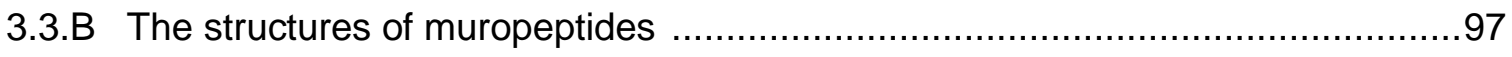

3. 4. Relative levels of muropeptides in whole-cells ..............................99

3.5. Relative levels of muropeptides in periplasm ......................................... 100

3.6. Relative levels of monosaccharides in whole-cell and periplasm ....................101

3.7. Relative levels of larger muropeptides in whole-cell and periplasm. .................102

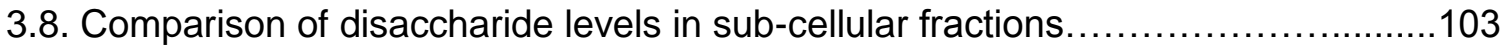


4.1. Amino acid sequence alignment of PA1085 with S. enterica FlgJ.

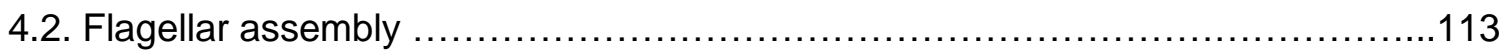

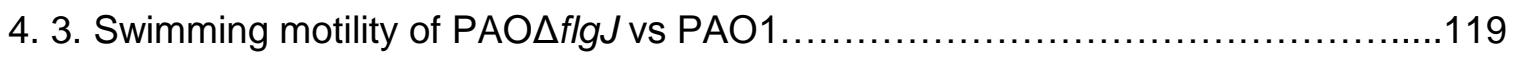

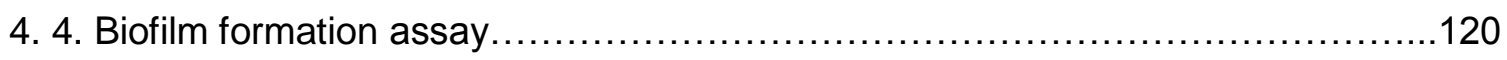

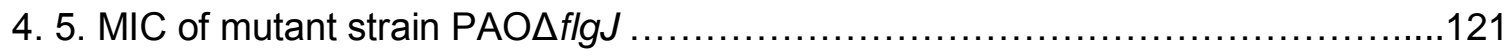

4. 6. $\mathrm{N}$-acetylglucosaminidase activity in whole-cell extracts........................ 122

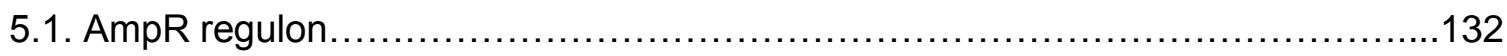

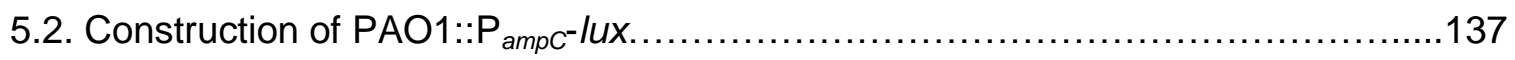

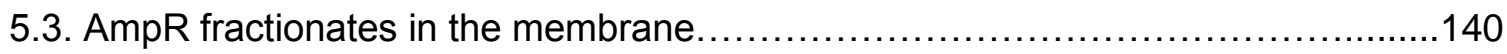

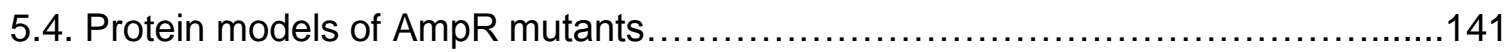

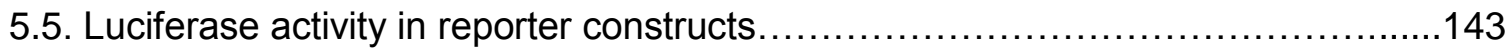

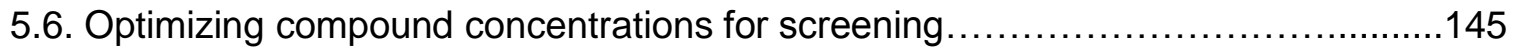

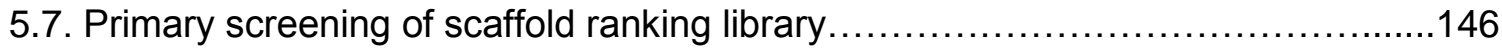




\section{LIST OF ABREVIATIONS AND ACRONYMS}

\begin{tabular}{|c|c|}
\hline $\mathrm{AHL}$ & $\mathrm{N}$-acyl Homoserine lactone \\
\hline AMP & Ampicilin \\
\hline anhMurNAc & anhydro $\mathrm{N}$-acetyl muramic acid \\
\hline ANOVA & Analysis of Variance \\
\hline ATM & Aztreonam \\
\hline BLAST & Basic local alignment search tool \\
\hline $\mathrm{bp}$ & Base pair \\
\hline $\mathrm{CAZ}$ & Ceftazidime \\
\hline CEF & Cefepime \\
\hline CF & Cystic Fibrosis \\
\hline CFU & Colony Forming Units \\
\hline CTX & Cefotaxime \\
\hline DAP & Diaminopimelic acid \\
\hline DNA & Deoxyribonucleic acid \\
\hline EDTA & Ethylenediaminetetraacetic acid \\
\hline ESBL & Extended-spectrum of P-lactamase \\
\hline et al. & et alia \\
\hline E-test & Epsilometer test \\
\hline FOX & Cefoxitin \\
\hline GlcNAc & $\mathrm{N}$-acetyl glucosamine \\
\hline $\mathrm{h}$ & Hour \\
\hline HMM PBPs & High Molecular Mass Penicillin Binding Protein \\
\hline
\end{tabular}




\begin{tabular}{ll} 
HPLC & High pressure liquid chromatography \\
HTH & Helix-tum-helix \\
IMI & Imipenem \\
IPTG & Isopropyl-D-I-thiogalactopyranoside \\
LB & Luria Bertani \\
LC/MS & Liquid chromatography/Mass spectrometry \\
LTs & Lytic transglycosylases \\
LTTR & LysR-type transcriptional regulator \\
MDR & Multi drug resistant \\
MER & Meropenem \\
MFS & Major facilitator superfamily \\
MIC & Minimum inhibitory concentration \\
mI & Milliliter \\
mm & Millimeter \\
mM & Millimolar \\
$4-M U G l c N A c$ & 4-methylumbelliferyl N-acetyl-D Glucosaminide \\
MurNAc & N-acetyl muramic acid \\
NaCl & Sodium chloride \\
NADH & Nicotinamide adenine dinucleotide \\
nm & nanometer \\
OD & Optical Density \\
ORF & Open reading frame \\
PCR & Polymerase chain reaction \\
PIP & Piperacilin \\
\hline
\end{tabular}




$\begin{array}{ll}\text { PQS } & \text { Pseudomonas quinolone signaling } \\ \text { QS } & \text { Quorum sensing } \\ \text { RNA } & \text { Ribonucleic acid } \\ \text { RND } & \text { Resistance nodulation division } \\ \text { T3SS } & \text { Type III secretion system } \\ \text { T6SS } & \text { Type VI secretion system } \\ \text { UDP } & \text { Uridine-Diphosphate } \\ \text { UTI } & \text { Urinary Tract Infection } \\ \text { XDR } & \text { Extensively drug resistant }\end{array}$




\title{
CHAPTER 1
}

\author{
Introduction
}

This chapter has been partly published:

Cell-wall recycling and synthesis in Escherichia coli and

Pseudomonas aeruginosa - their role in the development of resistance Dhar S, Kumari H, Balasubramanian D, Mathee K.

J Med Microbiol. 2018 Jan; 67(1):1-21. doi: 10.1099/jmm.0.000636. 


\subsection{Pseudomonas aeruginosa}

Pseudomonas aeruginosa is a Gram-negative bacteria of the family Pseudomonadaceae (Kapatral V, 2000). Members of this genus were described the first time by Walter Migula in late 1800 (Palleroni, 2004). The name was coined as "Pseudo" meaning false and "monas" refers to a unit (Palleroni, 2010). The species "aeruginosa" means copper rust or green. This alluded to the characteristic appearance of the bacteria in a culture owing to its synthesis of pigments such as pyocyanin and pyoverdin (Reyes, et al., 1981, Cox \& Adams, 1985). This organism is found ubiquitously, meaning in the earth, vegetation, soil, and water. It also has the capacity to survive on surfaces associated with medical devices like catheters and other instrument associated with dialysis (Talsma, 2007).

The ubiquity of these bacteria is due to its non-fastidious nature. It is well-known that $P$. aeruginosa can survive in minimal and versatile nutrition which contribute to the pervasive nature of this organism (Palleroni, 2001). Experiments have verified the presence of these bacteria even in distilled water containing minerals (Favero, et al., 1971, Legnani, et al., 1999). P. aeruginosa has been characterized as an obligate aerobe which can grow in anaerobic conditions with nitrate as an alternate electron acceptor (Carlson \& Ingraham, 1983, Davies, et al., 1989). It is both catalase and oxidase positive which is a distinguishing feature considered during lab. diagnosis and identification of Pseudomonal infections (Lysenko, 1961). 


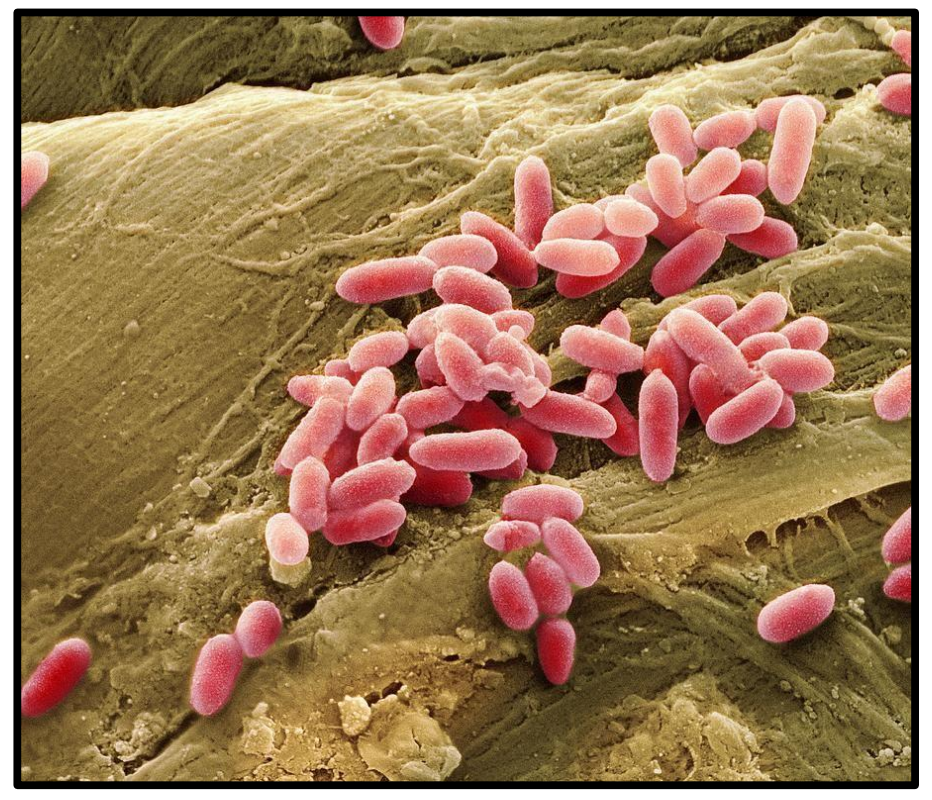

Figure 1.1. Pseudomonas aeruginosa bacteria (SEM). These Gram-negative, rodshaped bacteria are ubiquitous, often found in humans as part of the normal flora. They are also one of the common causes of hospital-acquired infections. (https://fineartamerica.com/featured/3-pseudomonas-aeruginosa-bacteria-semsemsteve-gschmeissner.html

The complex mechanisms that lie beneath its virulence are also reflected in its large genome size. The prototypic, lab reference strain of $P$. aeruginosa PAO1 has a genome size of more than 6 million base pairs with 5570 open reading frames (Holloway, 1955). In addition to PAO1, other sequenced strains include Liverpool Epidemic Strain LESB58, PA14, PACS2, C3719, and PA2192 (Cheng, et al., 1996, Lee, et al., 2006, Mathee, et al., 2008, Poirel, et al., 2010). A large set of genes that are conserved among these strains are referred to as the core genes (Mathee, et al., 2008). Besides this, the accessory genome consists of components that include transcriptional regulators, genes encoding for two-component systems and genes regulating/involved in antibiotic resistance as well as the regions of genome plasticity (Mathee, et al., 2008, Klockgether, et al., 2011). Comparative analysis of the different Pseudomonas species including $P$. 
aeruginosa, $P$. syringae, $P$. fluorescens, $P$. stutzeri, and $P$. putida, also highlight the contribution of horizontal gene transfers and genomic recombination to the versatility and adaptation that is exhibited by this pathogen (Silby, et al., 2011). All of the above enable the bacteria to survive, adapt and flourish under hostile conditions often found in the host, resulting in infections.

Besides the metabolic versatility and minimal nutritional requirements other factors that contribute to the ubiquitous nature of this bacteria as well as make this a formidable pathogen in clinical settings are the arsenal of virulence factors (Gellatly \& Hancock, 2013). Some of the common infections include hospital-acquired infections of the lung (Crouch Brewer, et al., 1996, Lyczak, et al., 2000), burn-wound (Estahbanati, et al., 2002, Dale, et al., 2004), urinary tract (Bouza, et al., 2002, Lamas Ferreiro, et al., 2017), and cancer chemotherapy (Vento, et al., 2008). It is also the primary pathogen responsible for morbidity and mortality in cystic fibrosis (CF) (Hoiby, 1974, Marks, 1981, Davies, 2002) chronic keratitis (Dart \& Seal, 1988) and otitis media and externa (Seyfried \& Fraser, 1978, Yeo, et al., 2007). Two main networks which are often interconnected underlie the severity of these infections; the arsenal of virulence factors and the variegated and numerous resistance mechanisms.

The initial stage of $P$. aeruginosa infection includes the process of bacterial adhesion to a surface followed by its colonization and subsequent invasion (Vasil, 1986). Remaining unchecked this may progress to a systemic infection. The virulence factors that are foundational for a successful infection can be broadly divided into those upregulated in chronic and others in acute infections stages. 


\subsection{Virulence in $P$. aeruginosa}

Flagella and Type 4 pili: $P$. aeruginosa flagellum and pili serve as a virulence factor providing motility and surface adhesion. Flagella are involved in maintaining swimming motility (Kearns, 2010). The absence of flagellum was found to result in strains that are deficient in acute infection (Feldman, et al., 1998). Along with pili, flagella also maintain swarming movements (Kohler, et al., 2000).

$P$. aeruginosa has multiple pili located at the polar ends. Both flagella and pili assist in adhesion by binding to receptors asialoGM1 which is expressed on the host epithelium especially upon a tissue breach (Gupta, et al., 1994, Feldman, et al., 1998).

Pili is responsible for the third type of movement shown by $P$. aeruginosa; twitching (Bradley, 1980). Flagella and pili are also a significant player in forming biofilms (O'Toole \& Kolter, 1998). Due to their role in $P$. aeruginosa pathogenesis, both flagella and pili have been targeted for anti-bacterial activity (Potvin, et al., 2003, Kipnis, et al., 2006).

Alginate: $P$. aeruginosa in presence of a stress such as host-response often turn mucoidy due to the production of an exopolysaccharide known as alginate (Evans \& Linker, 1973). This is composed of repeating units of D-mannuronic acid and L-guluronic acid (Remminghorst \& Rehm, 2006). This phenotype is predominantly seen in chronic infections especially in CF (Doggett, et al., 1964, Hoiby, 1974). Alginate helps in maintaining bacterial adhesion by preventing phagocytosis and opsonization (Bayer, et al., 1991, Pedersen, 1992). It also enhances the binding of the bacteria to lung epithelium. In addition, elimination of these mucoid strains proves more difficult as this slime-like substance forms an effective barrier against antibiotics. Mucoid strains of $P$. aeruginosa were found to be more resistant to aminoglycoside as well as $\beta$-lactams 
(Ramsey \& Wozniak, 2005). It was presumed that alginate formed the main matrix in biofilms, however that was not the case (Stapper, et al., 2004).

Lipopolysaccharide: The lipopolysaccharide (LPS) is an important immunologic component of $P$. aeruginosa (Pier, 2007). Although a lot of variabilities exists among its structure, its basic components include Lipid A, a core oligosaccharide and Ooligosaccharide or -antigen (King, et al., 2009). O-antigen of the LPS specifies the type of antibody that is produced against the bacteria and is the basis of serotyping (Kaya, et al., 1989). Lipid $A$ is recognized by the cells of the host immune system, such as Toll like receptor 4, which induces the release of cytokines resulting in inflammation (Backhed, et al., 2003, Pier, 2007).

Biofilm: $P$. aeruginosa exists in organized communities wherein they are embedded in a heteropolymer mesh composed of extracellular polysaccharides namely Pel and Psl, protein, DNA with interweaving water channels (Ma, et al., 2012, Jennings, et al., 2015). This is typically found in chronic infections and it increases the severity and tenacity of the infection (Costerton, et al., 1999, Bjarnsholt, 2013). Besides localizing on natural surfaces like respiratory airways, heart valve; biofilms often form on medical devices such as a catheter or stents (Tacconelli, et al., 2009). Infections found in biofilms are more difficult to eradicate resulting often in antibiotic resistance (Hoiby, 1974).

Type 3 secretion system (T3SS): One of the critical regulators of acute infection phenotypes is the T3SS (Hauser, 2009). Activation of this system depends upon hostcell contact and availability of calcium in the extracellular milieu (Frank, 1997, Vallis, et al., 1999). In $P$. aeruginosa, there are 43 genes distributed amongst the five operons that cluster together and a master-regulator ExsA regulates the activity of T3SS (Yahr \& Frank, 1994, Frank, 1997). This system is characterized by the production of toxic 
effector proteins which are secreted into the host-cytosol through a needle-like appendage similar to the flagellum. It comprises of four major effector proteins including ExoS, ExoT, ExoU, and ExoY (Frank, 1997). Three proteins PopB, PopD, and PcrV form the pore of the host cell membrane and assemble the secretory appendage to translocate the effectors. Effectors ExoS, $T$, and $U$ prevent endocytosis and phagocytosis as well as disrupt the epithelial barrier of the host tissue (Heimer, et al., 2013, Rangel, et al., 2015). ExoU also increases the release of proinflammatory eicosanoids and is often associated with bacteremia, sepsis and lung damage (McMorran, et al., 2003, Saliba, et al., 2005). ExoY is an adenylate cyclase that increases the intracellular cyclic AMP casing cell necrosis and barrier disruption (Stevens, et al., 2014). Animal models of infections including pneumonia; burn wounds, sepsis and keratitis were shown to be worsened in the presence of an active T3SS (Finck-Barbancon, et al., 1997, Sawa, et al., 1998, Lee, et al., 2003, Koh, et al., 2005).

Quorum Sensing (QS): As the name suggests, a process that depends upon the formation of a "quorum"; a minimum number of bacterial cells (Nealson \& Hastings, 1979). In other words, it is dependent on cell-signaling that is active once a certain cell density has been reached. The cell-signaling is facilitated through the synthesis and secretion of small-molecules known as "quoromones", which often have an acylhomoserine backbone (Parsek, et al., 1999). Quorum sensing in P. aeruginosa was classically known to involve two main players, the Las and Rhl-associated genes (Koch, et al., 1991, Gambello, et al., 1993, Ochsner, et al., 1994, Pearson, et al., 1995). In addition, there are two other systems namely the Pseudomonas quinolone system (PQS) and the relatively recently identified IQS (Pesci, et al., 1999, Lee, et al., 2013). Earlier studies show these systems are arranged in a hierarchy with the Las system at the top. However, more recent studies have suggested that the Rhl system may also be 
regulating the Las genes under certain conditions (Dekimpe \& Deziel, 2009). The IQS system is known to be functional only under phosphate starvation (Lee, et al., 2013). All the systems regulate virulence factors that can overwhelm the immune system of the host resulting in infection.

QS is involved in the production of pigments known as pyocyanin (Dietrich, et al., 2006). This is a phenazine compound that helps the bacteria to establish an infection in a CF patient's lungs (Kong, et al., 2006, Caldwell, et al., 2009, Carlsson, et al., 2011). It can also act as an antimicrobial due to its production of superoxide radicals (Hassett, et al., 1992). Another pigment produced by $P$. aeruginosa is pyoverdine, a siderophore (Stintzi, et al., 1998). Iron is one of the critical components essential for maintaining proper cellular physiology and growth (Ochsner, et al., 2002). Siderophores are ironchelators which transport extracellular iron to the cell-surface receptors and make it easily available to the bacteria (Visca, et al., 2007).

Besides the pigments, QS is also involved in regulating other virulence factors such as the production of a staphylolytic protease known as LasA, as well as elastase (LasB) production (Kessler, et al., 1993, Whiteley, et al., 1999). Both LasA and LasB are proteases which cause tissue invasion and have immune-modulatory properties (Kharazmi, 1989, Hobden, 2002). In addition, QS regulates the production of bacterial surfactants known as the rhamnolipids which play a prominent role in interfering with host-immune responses during an infection (Alhede, et al., 2009). These bio-surfactants prevent phagocytosis as well clearance of bacteria from the epithelial surface by impairing ciliary beat (Read, et al., 1992). Furthermore, motility and biofilm formation, which are under the influence of multiple genetic networks and environmental cues, are 
also regulated by QS (Kohler, et al., 2000, Davey, et al., 2003, Patriquin, et al., 2008, Solano, et al., 2014).

\subsection{Antibiotic resistance in $P$. aeruginosa}

Supporting the arsenal of virulence factors, $P$. aeruginosa infections are largely intractable due to the various mechanisms of resistance shown by this pathogen. Most commonly used antibiotic therapies include a combination of newer generation cephalosporins with aminoglycosides (Driscoll, et al., 2007, Kanj \& Kanafani, 2011). Despite this combination therapy, some infections remain unmanageable resulting in physicians resorting to the use of older antibiotics such as the polypeptide colistin which had previously been discontinued due to its nephro- and neurotoxic adverse events (Falagas \& Kasiakou, 2005). P. aeruginosa is listed as a pathogen of a serious threat (Level 2) by the US Center for Disease Control and Prevention as it causes 51,000 infections per year; of which 6,700 are multi-drug resistance with 440 deaths (CDC, 2013). Furthermore, carbapenem-resistant $P$. aeruginosa is also one of the three bacterial species listed by the World Health Organization as a pathogen of critical priority (World Health Organization, 2017). This bacterium is also one of the ESKAPE (Enterococcus faecium, Staphylococcus aureus, Klebsiella pneumoniae, cinetobacter baumannii, $\boldsymbol{P}$. aeruginosa and Enterobacter species) pathogens with growing multi-drug resistance(Figure 1.2.) (Rice, 2008).

The main mechanisms conferring resistance includes an impermeable outer membrane, expression of efflux pumps, alteration of drug target proteins and the production of hydrolytic enzymes. 


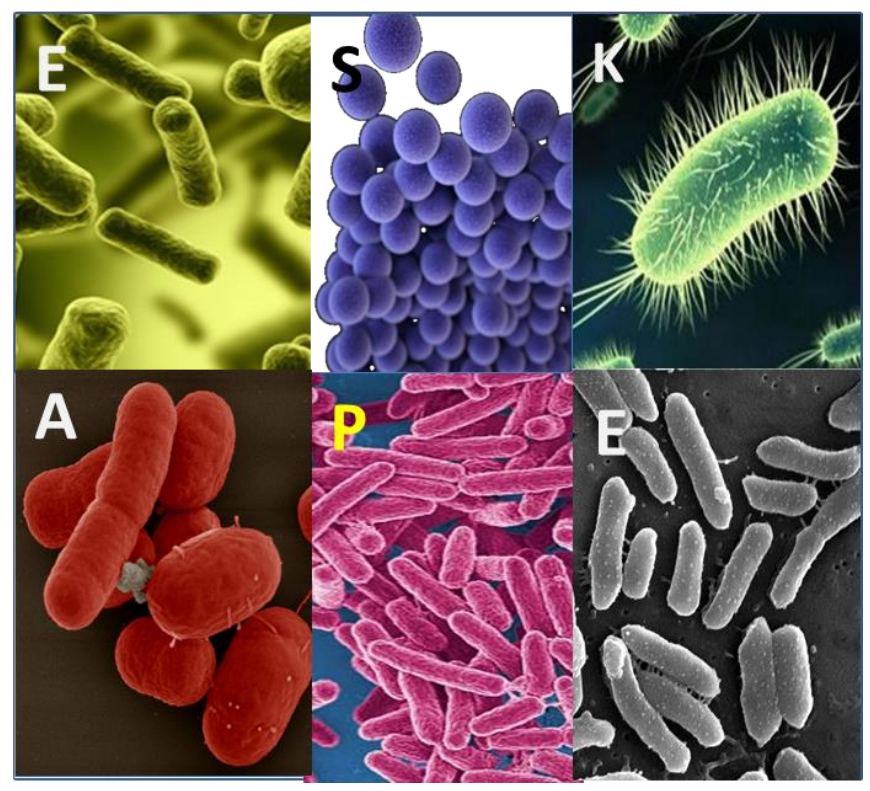

Figure 1. 2. The ESKAPE pathogens. These include a group of pathogens that show multi-drug resistance (MDR). It includes: E-Enterococcus faecium, S- Staphylococcus aureus, K- Klebsiella pneumoniae, A-Acinetobacter baumanii, P- Pseudomonas aeruginosa, E-Enterobacter species.

Figure adapted from: http://www.bacteriainphotos.com/

\subsubsection{Permeability barriers}

The outer membrane found in Gram-negative organisms forms the first line of defense against host-immune stimuli as well as from antibiotics. Most of the antibiotic targets are located in the cytoplasm of the bacteria and in order to reach the site of action, the antibiotics must first cross the outer membrane. Movement of smallmolecules across this barrier is facilitated by the presence of multiple channels located on the outer-membrane called as porin proteins.

Porin protein: The major porin found in $P$. aeruginosa is OprF (Hancock, et al., 1979, Nikaido, et al., 1991). Despite its abundance in the outer membrane, the intake of small molecules through this porin is far lesser when compared to OmpF, the major porin 
found in E. coli, (Yoshimura, et al., 1983). It has been shown that OprF has an open and a closed conformation (Sugawara, et al., 2006, Li, et al., 2012). At most times, a small number of the porin channels are open which accounts for the decreased permeability of P. aeruginosa to small molecules (Yoshimura \& Nikaido, 1982). Despite this, OprF is one of the most significant players' regulating small molecule uptake into $P$. aeruginosa (Nicas \& Hancock, 1983). Depletion of OprF from the outer membrane gives rise to increased resistance for fluoroquinolones and certain $\beta$-lactams such as ceftazidime and carbenicillin. However, the overall effect on resistance is often minimized due to the loss of membrane integrity in OprF mutants which creates an increased permeability to small molecules (Gotoh, et al., 1989). In addition to its role in antibiotic resistance, OprF has been found to be required for the pathogenesis of $P$. aeruginosa PAO1 and PA14 through modulation of quorum sensing and T3SS phenotypes (Fito-Boncompte, et al., 2011).

Another resistance determining factor in the outer-membrane is the OprD porin in $P$. aeruginosa (Li, et al., 2012). Its absence is most commonly associated with the advent of carbapenem resistance. The identification of this porin is hence linked with the discovery of resistance to imipenem, which is considered as the last line of defense against resistant-pseudomonal infections. Besides carbapenems, OprD also transports basic amino acids such as arginine, lysine, and histidine as well as peptides (Trias \& Nikaido, 1990). Nutrient media containing these specific amino acids was found to increase the minimal inhibitory concentration (MIC) of $P$. aeruginosa to carbapenems (Ochs, et al., 1999). This may be a concern in clinical settings wherein the presence of basic amino acids in human serum is not accounted for and hence the MIC obtained in vitro may not be truly reflective of the bacterial MIC in the patient's infection site. In the lab, it has been found that OprD expression can be increased when bacteria are grown 
with these amino acids as the sole source of carbon or nitrogen (Muramatsu, et al., 2003). The presence of trace metals like zinc and copper in the media causes downregulation of this channel (Perron, et al., 2004).

\subsubsection{Efflux-mediated resistance}

Another mechanism which is often found to act in synergy with an impermeable outer membrane is the expression of efflux pumps (Lambert, 2002). The efflux pumps are a predominant factor regulating antibiotic resistance and have been considered as good targets for novel antibiotics (Schweizer, 2003). The resistance-regulating efflux pumps found in Gram-negative bacteria including $P$. aeruginosa belong to the resistance nodulation-division (RND) family (Schweizer, 2003, Apel \& Surette, 2008). Structurally, these pumps can be divided into three major components wherein a channel is found on the outer membrane that is dependent on a proton motive force, an adaptor protein seen in the periplasmic area and an inner membrane transporter which captures and transports solutes from the cytoplasm to the extracellular milieu (Blair \& Piddock, 2009, Venter, et al., 2015).

$P$. aeruginosa has twelve efflux pumps which have been characterized. These include MexAB-OprM (Li, et al., 1995) and MeXY-OprM (Mine, et al., 1999), MexCDOprJ (Gotoh, et al., 1998), MexEF-OprN (Kohler, et al., 1997), MexJK (Chuanchuen, et al., 2002), MexGHI-OpmD (Aendekerk, et al., 2002), MexVW (Li, et al., 2003), MexPQOpmE (Mima, et al., 2005), MexMN (Mima, et al., 2005), TriABC (Mima, et al., 2007), MuxAB-OpmB (Mima, et al., 2009) and CzrAB-OpmN (Hassan, et al., 1999). These

efflux pumps have a broad and varied range of antibiotic substrates MexAB-OprM can 
expel the $\beta$-lactams including the antipseudomonal penicillins, the monobactams, cephlaosporins such as ceftazidime and cefotaxime as well as selected carbapenems such as meropenem and the $\beta$-lactamase inhibitors as well as fluoroquinolones and aminoglycosides (Li, et al., 1995, Srikumar, et al., 1998, Srikumar, et al., 2000) . Amongst the $\beta$-lactams, mostly the fourth generation of cephalosporins is transported by the MexCD-OprJ efflux pumps (Poole, et al., 1996). MexAB participates in regulating intrinsic resistance for $P$. aeruginosa whereas MexCD does not (Kohler, et al., 1996, Poole, et al., 1996). Carbapenems and cephalosporins are also transported by MexEFOprN (Kohler, et al., 1996, Kohler, et al., 1997). Fluoroquinolones can act as a substrate for most of the efflux pumps. Aminoglycosides such as gentamicin, amikacin, and tobramycin are the substrates of specifically MexXY-OprM (Poole, et al., 1996). It has been found that strains which exhibit multi-drug resistance often show overexpression of these pumps (Poole, et al., 1996, Oh, et al., 2003).

\subsubsection{Target modification.}

The sites of action of the $\beta$-lactams are the penicillin-binding proteins which are enzymes essential for the peptidoglycan (PG) synthesis. The $P G$ of bacteria is composed of two sugar units, $\mathrm{N}$-acetyl glucosamine, and $\mathrm{N}$-acetyl muramic acid. A five amino acid peptide chain composed of L-alanine, $\gamma$-D-glutamic acid, mesodiaminopimelic acid (meso-DAP), and D-alanyl-D-alanine is attached to the muramic acid (Heilmann, 1972, Heilmann, 1974). The penicillin-binding proteins catalyze an essential step in the formation of the cell-wall by acting as a transpeptidase and facilitate the cross-linking of the glycan chain between the third amino acid of one to the fourth amino-acid of another. The $\beta$-lactams act by binding covalently to the active site of the PBPs to form acyl-enzyme complexes (Wise \& Park, 1965, Bycroft \& Shute, 1985). 
Mutations occurring in this active site hence prevent the binding of the antibiotic to the enzyme. Besides specific mutation of the PBP that incapacitates drug-binding, the deletion of PBP4 was shown to result in high level expression of ampC resulting in $\beta$ lactam resistance in a clinical setting (Moya, et al., 2009). These specific mutants were also isolated from clinically resistant strains (Moya, et al., 2009). Decreased binding of $\beta$ lactam to PBP3 has been reported in $P$. aeruginosa treated for a long duration with tobramycin and antipseudomonal $\beta$-lactam (Godfrey, et al., 1981).

\subsection{4 $\beta$-lactamase mediated resistance.}

These are hydrolytic enzymes that target the $\beta$-lactam antibiotics. These enzymes were first identified for their role even before the global widespread of the first $\beta$-lactam i.e. penicillin (Abraham \& Chain, 1988). Given the diversity of the enzymes, they have been classified several times depending on their hydrolytic properties or substrate profile as well as any structural homology between the enzymes. Notable classifications amongst these include the scheme proposed by Richmond and Sykes who divided the Gram-negative enzymes into five major groups according to their substrate profile (Richmond \& Sykes, 1973). This was extended by Sykes and Matthew to include the plasmid-encoded enzymes (Sykes \& Matthew, 1976). Mitsuhashi and Inoue also categorized the enzymes according to their substrate profiles and included very specific hydrolytic activity such as those targeting a specific generation of cephalosporins (Mitsuhashi S, 1981). Two of the currently employed classifications are those by Ambler

and Bush. Bush modified and expanded the classification provided by Richmond and Sykes to include sub- groups for the enzymes based on substrates and inhibitor profile (Bush \& Singer, 1989, Bush, et al., 1995). The other classification scheme that is in use currently was proposed by Ambler and divides the enzymes into four groups A, B, C and 
D according to the conserved sequences amongst the amino acids (Ambler, 1980). Enzymes belonging to $A, C$, and $D$ classes have a serine in the active site and act by serine ester hydrolysis. Class B enzymes are also called metallo- $\beta$-lactamases and require a zinc ion in the active site for their activity (Nambu, et al., 1999).

Class A Enzymes: These enzymes are commonly plasmid-borne or encoded in the integron region with a minority of them being encoded on the chromosome (Matthew, 1979, Medeiros, 1997). The enzymes in this class are commonly known as penicillinases and demonstrate a broad substrate profile with respect to their hydrolytic properties (Matthew, 1979). The common groups of enzymes include TEM, SHV, PER, CTX-M, IMI, SME, GES, and VEB (Datta \& Kontomichalou, 1965, Matthew, et al., 1979, Nordmann, et al., 1993, Poirel, et al., 2000, Karim, et al., 2001, Zhao \& Hu, 2010, Fairfax, et al., 2011). $\beta$-lactamase inhibitors such as tazobactam, sulbactam, clavulanic acid, and relebactam have been found to inhibit their enzyme activities (Weldhagen, et al., 2003). TEM and its derivatives have been identified in Gram-negative pathogens including E. coli, K. pneumoniae, H. influenzae and N. gonorrhea (Ambler, 1980). Another common enzyme found in this class is the SHV. These are structurally homologous with TEM. Both TEM and SHV confer resistance to the synthetic and semisynthetic penicillins such as ampicillin, amoxicillin, ticarcillin, and carbenicillin as well as the narrow-spectrum cephalosporins (Livermore, 1995, Tzouvelekis \& Bonomo, 1999). Derivatives of these enzymes such as the TEM-1, TEM-2, and SHV-1 show extended spectrum providing resistance to $2^{\text {nd }}$ and $3^{\text {rd }}$ generation cephalosporins as well as monobactams (Weldhagen, et al., 2003). P. aeruginosa has derivatives TEM 21, 2442 and 4 along with SHV 2, 12 and 5 (Weldhagen, et al., 2003). In addition, clinical isolates with GES and its related IBC, VEB, and PER and their derivatives have also been recognized. The affinity is similar to TEM and SHV; they inhibit all the groups of $\beta$ - 
lactams including penicillins, cephalosporins, imipenem (Weldhagen, et al., 2003). In addition, GES-1 can also inhibit clavulanic acid, imipenem and as well as cefoxitin- a third generation cephalosporin (Poirel, et al., 2000). PER1- which has $20 \%$ amino acid identity with TEM and SHV also inhibits the monobactam (Bouthors, et al., 1998).

Class B Enzymes: Instead of a serine moiety in the active site, this enzyme class requires a zinc $\left(\mathrm{Zn}^{+2}\right)$ ion (Dufresne, et al., 1988, Palzkill, 2013). These enzymes are mainly expressed and transmitted through integrons in plasmids or transposons in pathogenic bacteria such as Enterobacteriaceae and $P$. aeruginosa (Palzkill, 2013). $P$. aeruginosa contains the IMP, VIM, SPM and GIM type of enzymes (Watanabe, et al., 1991, Lauretti, et al., 1999, Toleman, et al., 2002, Castanheira, et al., 2004). The IMP-, VIM-, and GIM-type enzymes are encoded within mobilizable units such as the plasmids and the transposons (Poirel, et al., 2000, Castanheira, et al., 2004, Brizio, et al., 2006). SPM-1 is closely associated with a genetic element known as ISCR (IS Common Region) that is related to the spread of antibiotic resistance (Toleman, et al., 2002). The enzymes in this class can degrade all the $\beta$-lactams except for aztreonam (Sacha, et al., 2008). Furthermore, these enzymes are also resistant to the $\beta$-lactamase inhibitors (Sacha, et al., 2008). There is a considerable homology between these enzymes and their derivatives. VIM derivative, VIM-1 shows less than $30 \%$ identity with the IMP-1 type enzymes whereas the SPM-1 shows $35.5 \%$ identity to IMP-1 (Lauretti, et al., 1999). GIM-1 also shows a high conservation with the IMP-1 enzyme (42\%), and slightly lesser with VIM-1 and SPM-1 (28-30\%) (Castanheira, et al., 2004).

Class C Enzymes: These enzymes are mainly chromosomal however plasmid-encoded forms have been detected (Papanicolaou, et al., 1990, Bauernfeind, et al., 1996, Walther-Rasmussen \& Hoiby, 2002). In P. aeruginosa two chromosomal class $C \beta$ - 
lactamases have been identified, AmpC and PIB-1 (Fajardo, et al., 2014). Overexpression of PIB-1 was found to confer resistance to carbapenems whereas AmpC overexpression led to increased resistance for all the groups of $\beta$-lactams (Fajardo, et al., 2014). The regulation of AmpC expression and its influence on $\beta$-lactam resistance is enumerated in detail later in the introduction.

Class D Enzymes: This group of enzymes is commonly known as oxacillinases owing to their ability to hydrolyze oxacillin, methicillin, cloxacillin, and dicloxacillin (Dale \& Smith, 1972). One of the recently identified enzymes in this group is PoxB, previously known as OXA-50 (Girlich, et al., 2004, Kong, et al., 2005). This enzyme is encoded in the chromosome and was found to have carbapenemase activity (Zincke, et al., 2016). In the presence of the primary chromosomally encoded $\beta$-lactamase AmpC, the effect of this enzyme on resistance is minimal. However, upon overexpression, this was found to render resistance to carbapenems. This effect was especially pronounced in the absence of the porin for carbapenems, OprD (Zincke, et al., 2016). The $\beta$-lactamase inhibitors (-bactam) do not inhibit this class of enzymes (Payne, et al., 1994). Other than this specific chromosomal $\beta$-lactamase, several plasmids encoded class D-enzymes has also been characterized. Some of these in $P$. aeruginosa are OXA-2 and its variants (15, -36), $-5,-6,-10$ and its variants (OXA-11,-14,-16,-17 and -19), and -20 (Danel, et al., 1995, Danel, et al., 1998, Mugnier, et al., 1998, Danel, et al., 1999, Poirel, et al., 2010). Additionally, another enzyme LCR-1 is also found which includes penicillin in its spectrum (Yang \& Bush, 1995).

\subsection{Regulation of AmpC $\beta$-lactamase expression.}

The expression of AmpC $\beta$-lactamase is inducible in many bacteria including $P$. aeruginosa (Lodge, et al., 1990). The study of AmpC regulation was first demonstrated 
in E. coli using C. freundii AmpR (Lindberg \& Normark, 1987). The connection of $\beta$ lactamase induction with recycling was realized after demonstrating that particular genes which participate in recycling also affected $\beta$-lactamase induction (Wiedemann, et al., 1998). This specific induction phenomenon was found to occur only in the presence of $\beta$ lactams, which led to an accumulation of muropeptides. As studied in Enterobacteriaceae, besides AmpR, other players that are required in this process include AmpG, AmpD, AmpDh2, AmpDh3, NagZ among others (Wiedemann, et al., 1998). As this process, has not been investigated in $P$. aeruginosa the next few sections entail the details of this process in this pathogen and compares it to the model organism that was used to study recycling- E. coli.

\subsection{Cell-wall recycling and synthesis in Escherichia coli and $P$. aeruginosa}

The cell-wall is an essential component of bacterial architecture that confers cell shape, prevents lysis under fluctuating internal turgor pressure, and protects from external assaults, thus enabling their ubiquitous existence. The cell-wall is composed of tightly cross-linked peptidoglycan (PG) which encircles the inner membrane and forms a protective layer known as the murein sacculus (Weidel \& Pelzer, 1964). The murein sacculus varies in size and chemical content in distinct species. In Gram-negatives such as $E$. coli, the cell-wall is approximately six nanometers $(\mathrm{nm})$, whereas in the Grampositives it may span up to $80 \mathrm{~nm}$ providing the capacity to withstand higher amounts of turgor pressure (Whatmore \& Reed, 1990, Matias, et al., 2003, Matias \& Beveridge, 2007). Extensive remodeling of the cell-wall occurs during bacterial cell growth and division. Thus, recycling of the cell-wall components is critical to conserve resources. Both Gram-positive and -negative bacteria recycle almost $40-50 \%$ of the cell-wall components (Goodell, 1985, Doyle, et al., 1988). Cell-wall recycling and synthesis are 
tightly coordinated to preserve bacterial integrity. Since the survival of bacteria critically depends on their PG-based cell-walls, it is a selective target of many antibiotics. Hence, the development of antibiotic resistance in bacteria is intimately tied in with the cell-wall synthesis and recycling (Jacobs, et al., 1994). Both these processes have been studied in Citrobacter freundii, Enterobacter cloacae, and E. coli and to some extent in P. aeruginosa (Jacobs, et al., 1994, Jacobs, et al., 1997, Lee, et al., 2016, Lee, et al., 2016).

$P$. aeruginosa infections are a serious clinical challenge due to their multiple antibiotic resistances and aggressive virulence mechanisms and its ability to establish persistent chronic infections. A dearth of antibiotics to treat multi-drug resistance threatens to cast the world back into a pre-antibiotic era (Davies \& Davies, 2010). P. aeruginosa shows both intrinsic as well as acquired resistance towards multiple classes of antibiotics (Lambert, 2002, Lister, et al., 2009). Intrinsic resistance to a widely used group of drugs, the $\beta$-lactams, is often conferred through the inducible $\beta$-lactamase AmpC, a process which interweaves with the cell-wall recycling pathway (Normark, 1995). Despite the discovery of cell-wall recycling in E. coli in the 1980's, technical limitations have hindered its full characterization in other organisms.

This review provides a brief overview of de novo cell-wall biosynthesis and focuses on what is known to date regarding cell-wall recycling in $E$. coli and $P$. aeruginosa. We discuss the roles of the genes involved in cell-wall synthesis and recycling in conferring resistance to both $\beta$-lactam and non $\beta$-lactam antibiotics. The review also focuses on the global regulator AmpR that connects cell-wall recycling to other resistance mechanisms through its diverse regulatory role in $P$. aeruginosa (Balasubramanian, et al., 2012, Kumari, et al., 2014). 


\subsubsection{Cell-wall biochemistry}

The bacterial murein sacculus is composed of a heteropolysaccharide of $\mathrm{N}$-acetyl muramic acid (MurNAc) and $N$-acetyl-glucosamine (GlcNAc) linked by $\beta-1 \rightarrow 4$ glycosidic linkages, with a short chain peptide containing up to five amino acids, attached to the muramyl moiety (Salton, 1953, Perkins, 1963, Schleifer \& Kandler, 1972). The full-length pentapeptide side chain in Gram-negative bacteria is typically composed of L-Alanine- - D-Glutamate-meso-diaminopimelic acid-D-Alanyl-D-Alanine (L-Ala- $\gamma$-D-Glu-m-DAP-DAla-D-Ala) (Glauner, et al., 1988). Cross-linking in Gram-negative bacteria between two adjacent peptide chains commonly occurs between the $3^{\text {rd }}$ residue of one chain ( $m$-DAP) and the $4^{\text {th }}$ residue (D-Ala) of the other (Schleifer \& Kandler, 1972, Sauvage, et al., 2008). High-performance liquid chromatography analysis of purified murein sacculus has shown that the extent of crosslinking is comparable between E. coli and P. aeruginosa (Quintela, et al., 1995). This crosslinking creates a mesh-like structure, which confers murein sacculus the strength to withstand internal pressure.

The PG in many Gram-negatives including E. coli and P. aeruginosa has linked proteins known as Braun's lipoprotein (Braun \& Rehn, 1969, Braun \& Sieglin, 1970, Quintela, et al., 1995). The covalent linkage of this lipoprotein between the outer membrane and the murein sacculus contributes further to the stability of the cell-wall. There is a considerable variation in the thickness of the murein sacculus amongst Gramnegative bacteria. For instance, the PG thickness of $E$. coli is $6.35 \pm 0.53 \mathrm{~nm}$ whereas $P$. aeruginosa is at $2.41 \pm 0.54 \mathrm{~nm}$ (Matias, et al., 2003). Despite the variations found in the details of the structural architecture in the Gram-negatives, the cell-wall is highly conserved across bacteria promoting its use as a valuable antibiotic target. 


\subsubsection{Cell-wall biosynthesis}

The biosynthesis of the cell-wall can be divided into three phases based on the localization of the processes; namely, those occurring in the cytoplasm, the inner leaflet of the cytoplasmic membrane, and the periplasm (Figure1. 3).

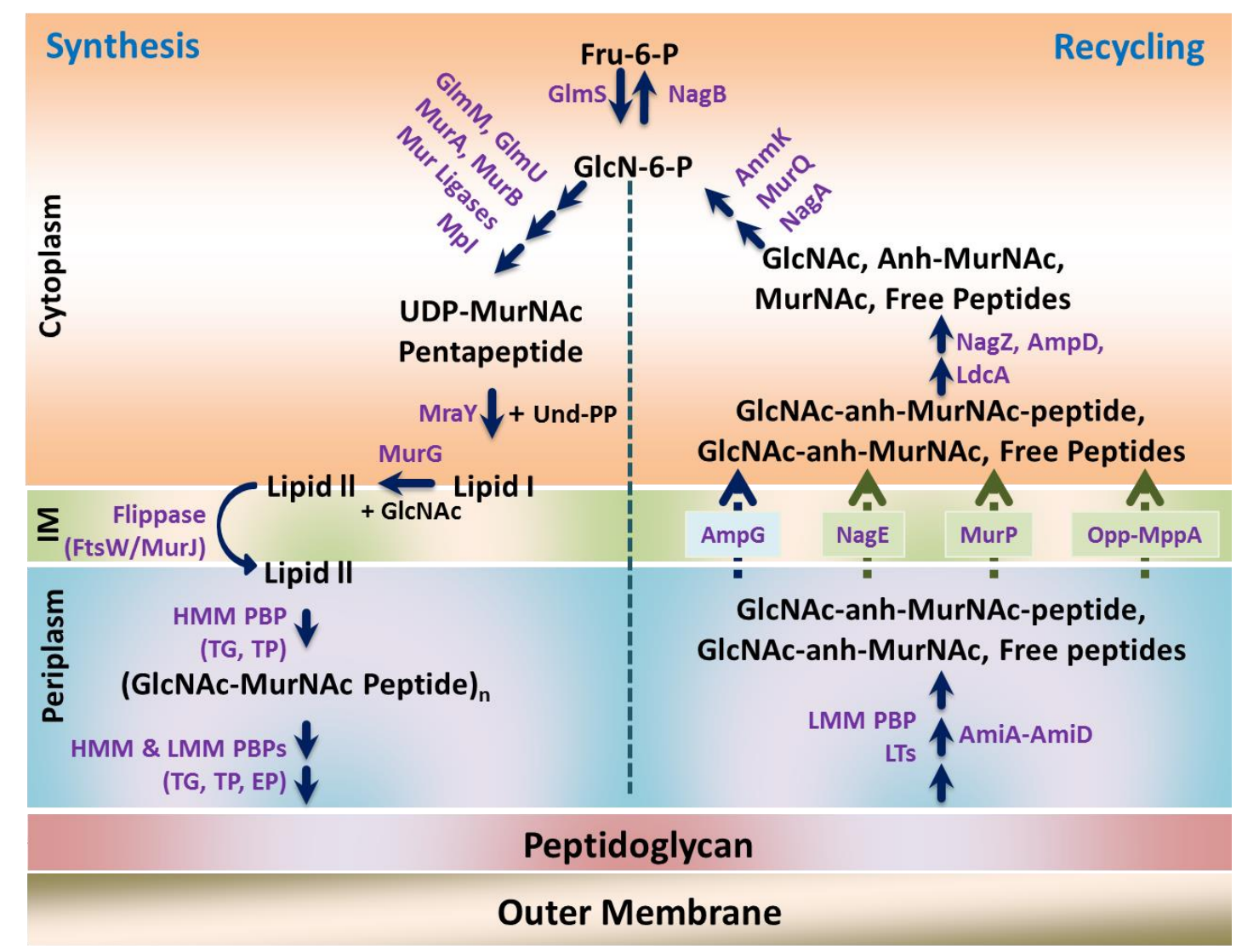

Figure 1.3. Peptidoglycan recycling and synthesis pathway in E. coli. The major cell-wall recycling enzymes found in the periplasm include the low molecular mass penicillin binding proteins (LMM PBPs: PBP4, PBP5, PBP6, PBP7/8), amidases (AmiAAmiD), and the lytic transglycosylases (LT: MltA, MltB, MltC, MltD, MltE, MltF, MltG, and Slt70). The major products formed are the $\mathrm{N}$-acetylglucosamine-1, 6-anhydro- $\mathrm{N}$ acetylmuramyl-peptides (GlcNAC-anhMurNAc-peptide) along with GlcNAc-anhMurNAc and free peptides. These muropeptides are translocated from the periplasm into the cytoplasm, mainly through a permease AmpG. Some of the muropeptides are also translocated through the OppBCDF complex with muropeptide-binding protein MppA, as well as through NagE and MurP. Once in the cytoplasm, these muropeptides are processed by $\beta$-N-acetylglucosaminidase (NagZ) and the $\mathrm{N}$-acetyl-anhydromuramyl-Lalanine amidase (AmpD) and an LD-carboxypeptidase (LdcA). The products are then acted upon by a kinase (AnmK), an etherase (MurQ), and a deacetylase (NagA) to form 
glucosamine-6-phosphate (GlcN-6-P). The recycling and biosynthesis pathways converge at this point. GlcN-6-P is converted back to fructose-6-phopshate (Fru-6-P) through a deaminase (NagB).

In the PG biosynthesis pathway, Fru-6-P is converted to GlcN-6-P through an amidotransferase (GlmS). The GlcN-6-P undergoes a series of modifications through a hexosephosphate mutase (GlmM), an uridyltransferase (GlmU), an enolpyruvyl transferase (MurA), a reductase (MurB) and the Mur ligases (MurC, MurD, MurE, MurF) to form the uridine-diphosphate (UDP) MurNAc-pentapeptides. In addition to the Mur ligases is the murein peptide ligase (Mpl) which can catalyze the ligation of tri-, tetraand pentapeptides to MurNAc. A translocase (MraY) transfers the UDP-MurNAc peptide onto an undecaprenyl-pyrophosphate (UND-PP) group to form Lipid I which anchors the complex to the inner membrane (IM). A glycosyltransferase (MurG) catalyzes the attachment of GIcNAc to form lipid II. Lipid II is then flipped from the cytoplasm into the periplasm using "flippase" (FtsW and MurJ). Once in the periplasmic space, the high molecular mass penicillin binding proteins (HMM PBPs) with transglycosylase (TG) and transpeptidase (TP) activities polymerize and cross-link the muropeptides with the existing peptidoglycan (PG). LMM PBPs with endopeptidase (EP) activity cleave the existing PG layer, facilitating the insertion of new glycan strands.

\section{Escherichia coli}

\section{Cytoplasm}

Fructose-6-phosphate is converted to uridine-diphosphate (UDP)-GIcNAc through series of steps driven by the activities of the GlmS, GlmM, and GlmU (Badet, et al., 1987, Mengin-Lecreulx \& van Heijenoort, 1994, Mengin-Lecreulx \& van Heijenoort, 1996). UDP-GICNAc is further converted to UDP-MurNAc pentapeptide by the activity of the Mur group of enzymes (MurA, MurB, MurC-MurF ligases) (Benson, et al., 1993, Barreteau, et al., 2008). To this pentapeptide, a 55-C aliphatic chain called undecaprenyl pyrophosphate is attached by MraY (Bouhss, et al., 2004). This aliphatic moiety is attached to the inner face of the cytoplasmic membrane to form Lipid I (Chatterjee \& Park, 1964, Anderson, et al., 1965).

\section{Inner membrane}

MurG catalyzes the addition of GIcNAc to the MurNAc moiety of Lipid I to yield Lipid II (Mengin-Lecreulx, et al., 1991, Barreteau, et al., 2008). The Lipid II is flipped over into 
the periplasmic space using a 'flippase' enzyme (Pomorski \& Menon, 2006). The identity of "flippase" remains ambiguous and is the subject of current research (Meeske, et al., 2015, Ruiz, 2015, Elhenawy, et al., 2016). In E. coli, FtsW and MurJ have been independently shown to be the proteins responsible for translocation of the lipid-linked muropeptides from the cytoplasm into the periplasmic space (Ruiz, 2008, Mohammadi, et al., 2011, Sham, et al., 2014, Leclercq, et al., 2017). Furthermore, in E. coli the transport and subsequent polymerization of Lipid II by FtsW is regulated via PBP1B and PBP3 (Leclercq, et al., 2017).

\section{Periplasm}

The final steps of the PG synthesis involve the incorporation of the GlcNAc-MurNAc pentapeptide component of Lipid II into the rapidly evolving murein sacculus. High molecular mass penicillin binding proteins (HMM PBPs) with a transglycosylase domain (PBP1A, PBP1B, and PBP1C) facilitate the link between the MurNAc end of the Lipid II with the GlcNAc of the existing strand (Ishino, et al., 1980, Suzuki, et al., 1980, Tamura, et al., 1980, Nakagawa, et al., 1984, Schiffer \& Holtje, 1999, Terrak, et al., 1999). HMM PBPs with a transpeptidase domain (PBP1A, PBP1B, PBP1C, PBP2 and PBP3) catalyze the formation of cross-links between two peptide chains (Ishino, et al., 1980, Nakamura, et al., 1983, Asoh, et al., 1986, Goffin \& Ghuysen, 1998, Nguyen-Disteche, et al., 1998) (Table 1.1). Cross-linking is composed of two steps wherein the terminal DAla-D-Ala of the peptide chain attached to MurNAc is cleaved with the release of one alanine (Izaki, et al., 1966). The murein tetrapeptide so formed is known as the "donor" peptide. Subsequently, the terminal D-alanine of the tetrapeptide attaches to the mesodiaminopimelic acid of an "acceptor" peptide chain (Izaki, et al., 1966). 


\section{Pseudomonas aeruginosa}

Bioinformatics analyses suggest the presence of $E$. coli homologs in $P$. aeruginosa; however, the enzymatic characterization of many of the proteins is yet to be done (Table 1.1).

\section{Cytoplasm}

Sequence comparison reveals homologs of GImM (PA4749), GImS (PA5549) and GImU (PA5552) that aids in the synthesis of UDP-GlcNAc from fructose-6-phosphate (Table 1.1). In $P$. aeruginosa glucosamine-6-phosphate synthetase GlmS is under the regulation of a repressor GlmR (PA5550) (Ramos-Aires, et al., 2004). A transcriptional factor NagR regulates both GImS and GlmU in E. coli, highlighting the difference in regulation of PG saccharide GlcNAc between the two organisms (Plumbridge, 1995). In addition to protein homology, the functional activity of GlmM as a phosphoglucosamine mutase in P. aeruginosa has also been established (Tavares, et al., 2000). Homologs of the Mur enzymes that lead to the formation of UDP-MurNAc pentapeptide from UDPGlcNAc namely an enolpyruvyl transferase MurA (PA4450), a reductase MurB (PA2977), as well as the Mur ligases, MurC (PA4411), MurD (PA4414), MurE (PA4417), and MurF (PA4416) are also encoded in the $P$. aeruginosa genome (Table 1.1). The activity of the $P$. aeruginosa Mur enzymes was analyzed by providing the initial substrate for MurA, UDP-GIcNAc, and detecting the formation of the final product UDP-MurNAcpentapeptide (El Zoeiby, et al., 2001). The enzymatic properties of all the Mur ligases of P. aeruginosa were also heterologously characterized in E. coli (El Zoeiby, et al., 2000, Azzolina, et al., 2001). P. aeruginosa also encodes a homolog of Mpl ligase (PA4020) which facilitates ligation of tri-, tetra- and pentapeptides to UDP-MurNAc (Table 1.1). The cytosolic translocase MraY (PA4415) then transfers the UDP-MurNAc pentapeptide to 
an undecaprenol carrier group that anchors the muropeptide to the inner membrane (Table 1.1) (Azzolina \& El-Sherbeini, 2002). P. aeruginosa has homologs of inner membrane glycosyltransferase MurG (PA4412) and flippases FtsW (PA4413) and MviN (PA4562) (Table 1.1). A crystal structure of $P$. aeruginosa MurG has been solved in complex with its substrate, UDP-GlcNAc (Brown, et al., 2013).

\section{Periplasm}

The periplasmic cell-wall synthesis enzymes for polymerization and cross-linking of the muropeptides include the HMM PBPs, namely PBP1A (PA5045), PBP1B (PA4700), PBP2 (PA4003), and PBP3 (PA4418) (Table 1.1) (Noguchi, et al., 1979, Handfield, et al., 1997, Legaree, et al., 2007, Chen, et al., 2017). By sequence homology to E. coli, PBP1A (PA5045) and 1B (PA4700) contain both transglycosylase and transpeptidase domains whereas PBP2 (PA4003), and PBP3 (PA4418) have the domain for transpeptidase activity (Finn, et al., 2016, Marchler-Bauer, et al., 2017). In addition, $P$. aeruginosa also contains an additional transpeptidase, PBP3B/3X (PA2272), which is not found in E. coli (Liao \& Hancock, 1997). P. aeruginosa PBP3 and 3B expression differ temporally, the former being upregulated during the exponential and the latter during stationary growth-phase (Liao \& Hancock, 1997). 
Table 1.1. List of genes involved in cell-wall synthesis

\begin{tabular}{|c|c|c|c|c|c|}
\hline \multirow{2}{*}{ Proteins } & \multicolumn{2}{|r|}{ Genes } & \multicolumn{2}{|c|}{$\begin{array}{l}\text { Protein Homology } \\
(\%)\end{array}$} & \multirow{2}{*}{$\begin{array}{l}\text { Functional } \\
\text { Annotation }\end{array}$} \\
\hline & $\begin{array}{r}E . \\
\text { coli }\end{array}$ & $P$. aeruginosa & Identity $^{*}$ & $\underset{¥}{\text { Similarity }}$ & \\
\hline \multicolumn{6}{|c|}{ Periplasm } \\
\hline PBP1a & $\begin{array}{l}\text { ponA } \\
\text { mrcA }\end{array}$ & PA5045 & 41 & 58 & $\begin{array}{l}\text { Transglycosylase and } \\
\text { transpeptidase }\end{array}$ \\
\hline PBP1B & $\begin{array}{l}\text { ponB/ } \\
m r c B\end{array}$ & PA4700 & 38 & 56 & $\begin{array}{l}\text { Transglycosylase and } \\
\text { transpeptidase }\end{array}$ \\
\hline PBP1C & $p b p c$ & NF & - & - & $\begin{array}{l}\text { Transglycosylase and } \\
\text { transpeptidase }\end{array}$ \\
\hline MgtA & $m g t A$ & PA0378 & 48 & 65 & Transglycosylase \\
\hline PBP2 & $\begin{array}{l}p b p A / \\
m r d A\end{array}$ & PA4003 & 43 & 61 & Transpeptidase \\
\hline PBP3 & $f t s l / p b p B$ & PA4418 & 43 & 60 & $\begin{array}{l}\text { Transpeptidation. } \\
\text { Important for septa } \\
\text { formation during cell- } \\
\text { division }\end{array}$ \\
\hline PBP3b & $\mathrm{NF}$ & PA2272 & - & - & Transpeptidase \\
\hline PBP4 & $d a c B$ & PA3047 & 26 & 40 & $\begin{array}{l}\text { D-alanyl-D-alanine } \\
\text { carboxypeptidase and } \\
\text { endopeptidase }\end{array}$ \\
\hline PBP5 & dacA & PA3999 & 46 & 62 & DD-carboxypeptidase \\
\hline PBP6 & dacC & PA3999 & 46 & 65 & DD-carboxypeptidase \\
\hline PBP6b & dacD & $\mathrm{NF}$ & - & - & DD-carboxypeptidase \\
\hline PBP7 & $p b p G$ & PA0869 & 63 & 75 & $\begin{array}{l}\text { DD-carboxypeptidase } \\
\text { and endopeptidase }\end{array}$ \\
\hline \multicolumn{6}{|c|}{ Inner membrane } \\
\hline FtsW & $f t s W$ & PA4413 & 39 & 55 & Flippase (Putative) \\
\hline MurJ & murJ & PA4562 & 75 & 88 & Flippase (Putative) \\
\hline MurG & murG & PA4412 & 45 & 62 & $\begin{array}{l}\text { UDP-N- } \\
\text { acetylglucosamine-N- } \\
\text { acetylmuramyl- } \\
\text { pentapeptide } \\
\text { transferase } \\
\end{array}$ \\
\hline \multicolumn{6}{|c|}{ Cytoplasm } \\
\hline MraY & mraY & PA4415 & 67 & 81 & $\begin{array}{l}\mathrm{N} \text {-acetylmuramyl- } \\
\text { pentapeptide- } \\
\text { transferase }\end{array}$ \\
\hline GImM & $g / m M$ & PA4749 & 60 & 74 & $\begin{array}{l}\text { Phosphoglucosamine } \\
\text { mutase }\end{array}$ \\
\hline GImU & $g \operatorname{lmU}$ & PA5552 & 56 & 71 & $\begin{array}{l}\text { Glucosamine-1- } \\
\text { phosphate } \\
\text { acetyltransferase, } \\
\text { N-acetylglucosamine- } \\
\text { 1-phosphate }\end{array}$ \\
\hline
\end{tabular}




\begin{tabular}{|c|c|c|c|c|c|}
\hline & & & & & uridyltransferase \\
\hline MurA & murA & PA4450 & 60 & 74 & Enolpyruvyl transferase \\
\hline MurB & murB & PA2977 & 42 & 56 & $\begin{array}{l}\text { UDP-N-acetyl } \\
\text { pyruvylglucosamine } \\
\text { reductase }\end{array}$ \\
\hline MurC & murC & PA4411 & 58 & 73 & $\begin{array}{l}\text { UDP-N- } \\
\text { acetylmuramate L- } \\
\text { alanine ligase }\end{array}$ \\
\hline MurD & murD & PA4414 & 50 & 63 & $\begin{array}{l}\text { UDP-N-acetylmuramyl } \\
\text { L-alanine-D-glutamate } \\
\text { ligase }\end{array}$ \\
\hline MurE & murE & PA4417 & 47 & 58 & $\begin{array}{l}\text { UDP-N-acetylmuramyl } \\
\text { L-alanyl-D-glutamate- } \\
\text { diaminopimelate ligase }\end{array}$ \\
\hline MurF & murF & PA4416 & 46 & 60 & $\begin{array}{l}\text { UDP-N-acetylmuramy } \\
\text { L-alanyl-D-glutamyl- } \\
\text { diaminopimelate-D- } \\
\text { alanyl-D-alanine ligase }\end{array}$ \\
\hline Mpl & $m p l$ & PA4020 & 59 & 72 & Murein peptide ligase \\
\hline GImS & $g \operatorname{lmS}$ & PA5549 & 63 & 79 & $\begin{array}{l}\text { Glucosamine-6- } \\
\text { phosphate synthase }\end{array}$ \\
\hline
\end{tabular}

The data is compiled from EcoCyc and Pseudomonas Genome database website (Keseler, et al., 2009, Winsor, et al., 2016).

$¥$ The value for protein sequence identity and similarity of $E$. coli and $P$. aeruginosa was obtained using ClustalW2 "pairwise sequence alignments".

NF: Not Found

\subsubsection{Peptidoglycan growth}

To ensure bacterial growth (elongation and division) while maintaining cellular integrity, both PG degradative enzymes such as low molecular mass penicillin binding proteins (LMM PBP), lytic transglycosylases (LT), and amidases and PG synthase enzymes such as HMM PBPs must perform in a coordinated fashion along with the various bacterial cytoskeletal elements to avoid cell lysis (Typas, et al., 2011). The growth of the murein sacculus has been studied extensively in $E$. coli by Park et al 
(Burman, et al., 1983, Park \& Burman, 1985). Using radioactively-labelled PG they demonstrated that newly formed PG strands are cross-linked with the existing strand at multiple sites. At the same time, the cross-links in the existing murein strand are degraded by endopeptidases (Burman, et al., 1983). A three-for-one growth model suggests that the existing strand acts as a dock that incorporates three newly synthesized murein chains (Holtje, 1998). The soluble PG fragments that are generated during this progressive replacement of the existing murein with the new strand are recycled (Goodell \& Schwarz, 1985).

\subsubsection{Cell-wall recycling}

As the cells divide, the walls break and rejoin at every cycle, thus generating muropeptides of varying lengths. Gram-positives such as Bacillus sp., Lactobacillus acidophilus release close to $25-30 \%$ of their muropeptides into the culture media (Mauck, et al., 1971, Boothby, et al., 1973, Doyle, et al., 1988). Only 6-8\% extracellular muropeptides were detected for E. coli (Goodell \& Schwarz, 1985). The latter finding gave rise to the hypothesis that the Gram-negatives recycle their muropeptides. The hypothesis was confirmed by the detection of tritiated-DAP reuse by the bacteria during cell growth (Goodell, 1985). Extensive studies in E. coli have provided the knowledge on cell-wall recycling in Gram-negatives (Park \& Uehara, 2008) (Figure1. 4). Though $P$. aeruginosa harbors the orthologous genes, their functional roles remain to be elucidated (Kong, et al., 2010) (Table 1.2). This following section compares cell-wall recycling in Gram-negative E. coli and $P$. aeruginosa. The pathway is broken down into processes 
occurring in the subcellular compartments; namely the periplasm, inner membrane, and cytoplasm (Figure 1.4).

\section{Periplasm}

Mainly, three types of lytic enzymes are found in the periplasmic space that creates the muropeptide intermediates (Figure 1.3, 1. 4). These include the LTs, the LMM PBPs and the amidases (Park \& Uehara, 2008, van Heijenoort, 2011).

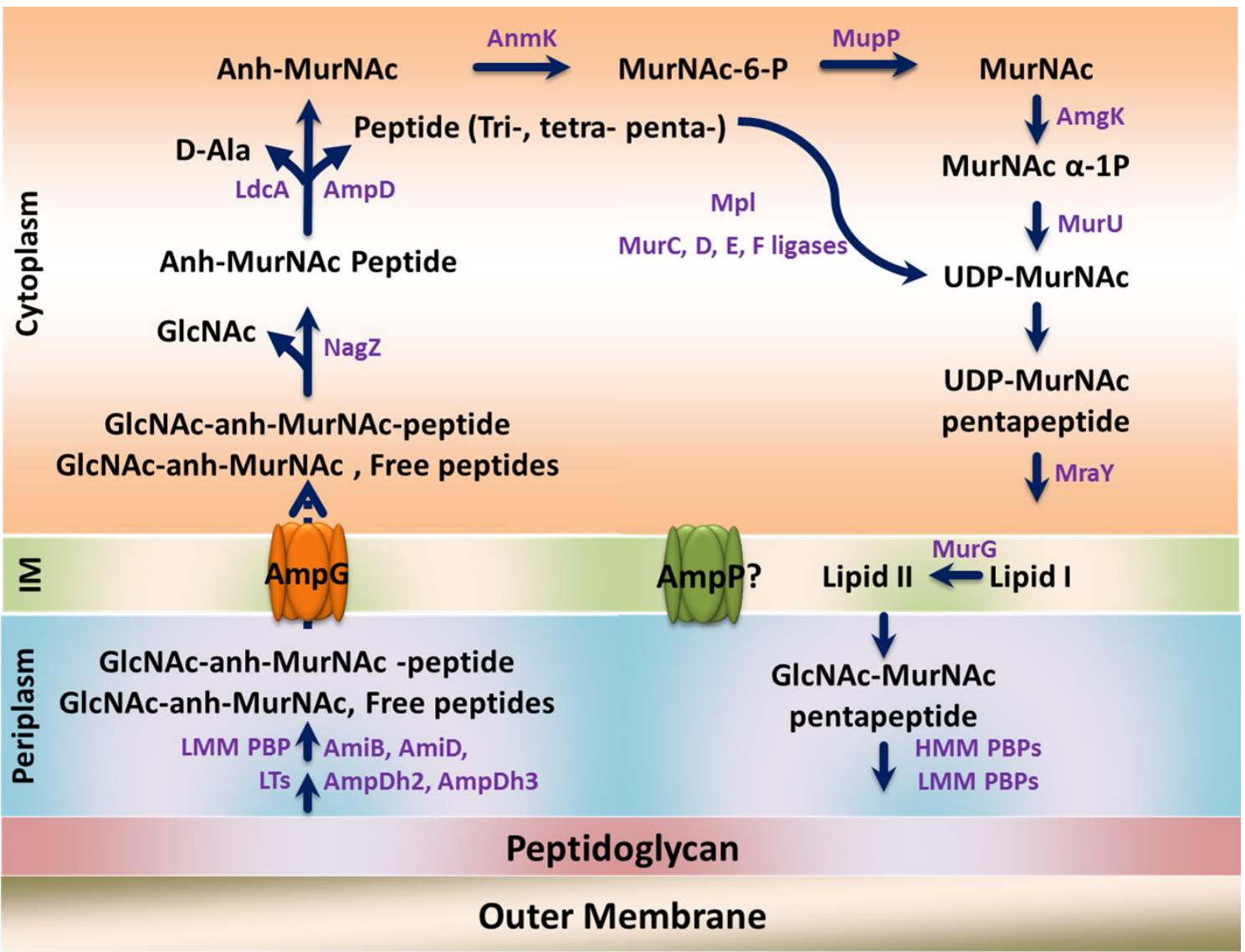

Figure 1. 4 Outline of cell-wall recycling in $\boldsymbol{P}$. aeruginosa. Muropeptides formed in the periplasm through low molecular mass penicillin binding proteins (LMM PBPs: PBP4, 
PBP, PBP6, PBP7/8), amidases (AmiB, AmiD, AmpDh2 and AmpDh3), and the lytic transglycosylases (LT: MltA, MltB, MltD, MltF, MltF2, MltG, Slt, SltB2, SltH/SltB, and RlpA) are internalized into the cytoplasm through a permease AmpG. AmpP is a paralog of AmpG found in $P$. aeruginosa whose function in cell-wall recycling has not been elucidated yet. Muropeptides imported by AmpG are subsequently degraded by a $\beta-\mathrm{N}$ acetylglucosaminidase (NagZ) which removes the GlcNAc moiety. The product Nacetyl-anhydro muramic acid (anh-MurNAc) peptide is processed by an N-acetylanhydromuramyl-L-alanine amidase (AmpD) which cleaves the peptide chain; and a LDcarboxypeptidase (LdcA) that removes the terminal amino acid D-alanine (D-Ala) from a tetrapeptide. In $P$. aeruginosa, anh-MurNAc is reutilized and converted to MurNAc-6phosphate (MurNAc-6-P) by a kinase (AnmK). MurNAc-6-P is then converted to MurNAC by a phosphatase (MupP). Following this, an anomeric MurNAc/GIcNAc kinase (AmgK) catalyzes the formation of MurNAc- $\alpha$-1-phosphate; to which an uridylyl transferase attaches a uridine-diphosphate (UDP) to facilitate the formation of UDPMurNAc. AmgK, MupP, and MurU cell-wall recycling enzymes are found exclusively in $P$. aeruginosa. Following the formation of UDP-MurNAc, the peptide chain is added by a murein peptide ligase (Mpl) or by Mur ligases (MurC, MurD, MurE and MurF). The UDPMurNAc pentapeptide is converted to Lipid I (undecaprenol-pyrophosphate-UDPMurNAc peptide) by a MurNAc-pentapeptide translocase (MraY) and a glycosyltransferase (MurG) then attaches the GlcNAc moiety to form Lipid II (undecaprenol-pyrophosphate-UDP-GIcNAc-MurNAc peptide). Lipid II is flipped into the periplasm through putative "flippases" (FtsW and MurJ). The GlcNAc-MurNAc peptide is reincorporated into the growing $P G$ through high molecular mass (HMM) and LMM PBPs.

\section{Lytic transglycosylases (LTs)}

The LTs act on the murein sacculus to release their signature product GlcNAc 1, 6 anhydro MurNAc (Holtje, et al., 1975) (Figure 1.4). The LTs facilitate PG remodeling, insertion of membrane-associated structures such as flagella and the secretion systems, and help in cell separation during division (Heidrich, et al., 2002, Koraimann, 2003, Scheurwater, et al., 2008). Investigation of the E. coli ORFs led to the identification of eight genes encoding LTs; mltA, mltB, mltC, mltD, mltE (emtA), mltF, mltG and slt70 (Holtje, et al., 1975, Engel, et al., 1992, Romeis, et al., 1993, Dijkstra \& Keck, 1996, Kraft, et al., 1998, Lee, et al., 2013, Yunck, et al., 2016). The enzymatic activities of all the $E$. coli LTs were demonstrated using the murein sacculus isolated from the log and stationary phase as a substrate (Lee, et al., 2013). All the LTs are exolytic and cleave at 
a terminus of the PG strand. However, MltE, MltD, MltB, Slt70 (SItY) and MltC are endolytic and cleave in the middle of a PG strand (Lee, et al., 2013). Of these, MltA was reported to have the highest activity on purified murein sacculus. All the enzymes show a higher activity on the murein sacculus isolated during the log phase than the stationary phase. The higher activity is in accordance with the extensive remodeling that occurs at the log phase (Lee, et al., 2013). It may also be due to the structural differences in the sacculus during the phases of bacterial growth as well as the lack of cell division during stationary phase (Glauner, et al., 1988). Deletion analyses demonstrate the presence of extensive functional redundancy within this group of eight genes encoding LTs (Heidrich, et al., 2002). E. coli tolerate the loss of up to three LTs, but deletion of all eight leads to cell death (Lommatzsch, et al., 1997, Kraft, et al., 1999, Heidrich, et al., 2002).

The LTs in $P$. aeruginosa were first identified using renaturing PAGE and zymograms (Watt \& Clarke, 1994, Li, et al., 1996). A total of 11 LTs, MltA (PA1222), MltB (PA4444), MltD (PA1812), MltF (PA3764), MltF2 (PA2865), MltG (PA2963), Slt (PA3020), SltB2 (PA1171), SltH/SItB3 (PA3992), and RIpA (PA4000) were reported in $P$. aeruginosa (Blackburn \& Clarke, 2001, Blackburn \& Clarke, 2002, Cavallari, et al., 2013, Jorgenson, et al., 2014, Lee, et al., 2017). A few studies separately enumerate the enzymatic activities of all the lytic transglycosylases (Jorgenson, et al., 2014, Lee, et al., 2016, Lee, et al., 2017). In vitro analyses with purified enzymes demonstrated that all the P. aeruginosa LTs have both endolytic and exolytic activity. MltD, MltF2, RIpA, and Slt exhibited higher endolytic activity whereas MltA, SltB1, SltB2, and SltB3 had higher exolytic activity. The most and least active enzymes are SltB1 and MltF2, respectively. Some of these enzymes exhibit substrate preference - MltB specifically requires a peptide chain on the muropeptide to demonstrate its lytic activity whereas RlpA acts on 
the degradation products of amidases, namely GlcNAc-anhMurNAc without the attached peptide chains (Jorgenson, et al., 2014, Lee, et al., 2017) (Figure 1.4).

\section{Low molecular mass penicillin binding proteins (LMM PBPs)}

The LMM PBPs act mostly as endopeptidases and or carboxypeptidases (Goffin \& Ghuysen, 1998, Sauvage, et al., 2008, Vollmer, et al., 2008). The endopeptidase activity of PBPs leads to hydrolysis of the cross-bridge between $m$-DAP and D-Ala, whereas the carboxypeptidases remove the terminal amino acid from a pentapeptide during crosslinking (Strominger, et al., 1967, Sauvage, et al., 2008, Vollmer, et al., 2008).

E. coli have five LMM PBPs, namely PBP4 (DacB), PBP5 (DacA), PBP6 (DacC), PBP6b (DacD) and PBP7/8 (PbpG) (Spratt \& Strominger, 1976, Holtje, 1998, Denome, et al., 1999, Kong, et al., 2010) (Table 1.2). PBP4/ DacB is a bifunctional peptidase with both carboxy and endopeptidase activities (Korat, et al., 1991). Together with PBP5 (DacA), PBP4 (DacB) is involved in maintaining normal cell morphology (Korat, et al., 1991, Meberg, et al., 2004, Clarke, et al., 2009). PBP5 (DacA) is found attached to the inner membrane (Spratt, 1977). It is the major carboxypeptidase in E. coli; deletion of $\operatorname{dac} A$ is associated with cell shape defects and a striking increase in some pentapeptides (Matsuhashi, et al., 1979, De Pedro, 1980, Nelson \& Young, 2000, Nelson \& Young, 2001). PBP6/DacC and PBP6b/DacD exhibit carboxypeptidase activities (Tamura, et al., 1976, Amanuma \& Strominger, 1980, Baquero, et al., 1996). An increase in expression levels of dacC is noted during the stationary phase (van der Linden, et al., 1992).

Another endopeptidase PBP7/PbpG and its proteolytic product PBP8 have been identified in E. coli (Henderson, et al., 1995). Loss of pbpG did not result in any 
morphological aberrations, however, the absence of both $d a c A$ and $p b p G$ encoding PBP5 and PBP7, respectively resulted in increased cell shape defects as compared to the loss of dacA alone (Romeis \& Holtje, 1994, Meberg, et al., 2004). Expression of $\operatorname{dac} A$ and $\operatorname{dacC}$ is regulated by the BolA protein, which is highly conserved in eukaryotes and prokaryotes (Santos, et al., 2002, Guinote, et al., 2011). BolA maintains cell morphology in both a PBP5/PBP6-dependent and -independent manner (Freire, et al., 2009). BolA regulates the carboxypeptidase activities of DacA/PBP5 and DacC/PBP6, and overexpression of bolA showed a decreased rate of growth which was lost upon deletion of both dacA and dacC (Santos, et al., 2002, Guinote, et al., 2011). Overexpression of bolA also impaired PBP2-mediated cell elongation and formation of cytoskeleton due to spatial disorganization of MreB (Freire, et al., 2009).

LMM PBPs in $P$. aeruginosa include PBP4/DacB (PA3047), PBP5/DacC (PA3999), and PBP7/PbpG (PA0869) (Noguchi, et al., 1979, Song, et al., 1998, Smith, et al., 2013, Lee, et al., 2015, Ropy, et al., 2015). Loss of dacC results in increased pentapeptides establishing its role as the primary carboxypeptidase in P. aeruginosa (Noguchi, et al., 1985, Smith, et al., 2013, Ropy, et al., 2015). P. aeruginosa encodes a homolog of BolA (PA0857), which shows a $46 \%$ identity to their E. coli counterpart. However, its role as a regulator of carboxypeptidases in $P$. aeruginosa is yet to be investigated. An additional increase of pentapeptides is also seen upon deletion of $d a c B$ and $p b p G$ confirming the presence of multiple carboxypeptidases (Ropy, et al., 2015). PBP4/DacB and PBP7/PbpG also act as endopeptidases (Song, et al., 1998, Lee, et al., 2015, Ropy, et al., 2015). P. aeruginosa appears to not harbor PBP6. However, P. aeruginosa PBP5 shares $48 \%$ homology with E. coli PBP6. 


\section{Amidases}

The periplasmic amidases remove the amino acid chain from the muramyl moieties of the sacculus as well as from the recycling by-products such as GlcNAc-anhMurNAc peptides (Van Heijenoort \& Van Heijenoort, 1971, van Heijenoort, et al., 1975, Tomioka, et al., 1983, Priyadarshini, et al., 2007, Uehara \& Park, 2007). E. coli expresses four periplasmic enzymes ami $A$, amiB, amiC, and amiD that have $\mathrm{N}$-acetylmuramyl-L-alanine amidase activity (Tomioka, et al., 1983, Tsui, et al., 1994, Heidrich, et al., 2001). AmiA, AmiB, and AmiC have a high sequence homology and assist in cell separation during division (Heidrich, et al., 2001). Loss of these three amidases affected septal formation resulting in a chaining phenotype with aggregates of three to six cells (Heidrich, et al., 2002, Priyadarshini, et al., 2007). The activity of these three enzymes is regulated spatiotemporally by proteins with LytM domains, which have conserved sequences and are collectively known as dLytM factors (Uehara, et al., 2009). EnvC and NIpD are dLytM proteins (Uehara, et al., 2009). EnvC belongs to the divisome group of proteins that participate in cytokinesis and activates AmiA and AmiB, whereas NIpD regulates AmiC activity (Uehara, et al., 2010). AmiD is tethered to the outer membrane and does not contribute to cell separation, and its loss has no effect on the cell-wall morphology (Uehara \& Park, 2007, Mercier, et al., 2010). AmiD cleaves at both $\mathrm{N}$-acetylmuramyl and 1, 6 anhydro- $\mathrm{N}$-acetylmuramyl peptides and plays a critical role in generating fragments for recycling (Priyadarshini, et al., 2007, Uehara \& Park, 2007, van Heijenoort, 2011). The loss of amiD resulted in an accumulation of GlcNAc-anhMurNAc tri- and tetrapeptides (Uehara \& Park, 2007).

P. aeruginosa has homologs of periplasmic AmiA (PA5538) and AmiB (PA4947). Both these proteins share homology with their E. coli counterparts and have $\mathrm{N}$ acetylmuramyl-L-alanine amidase activity (Scheurwater, et al., 2007). Deletion of amiA 
exhibited no significant effect on $P$. aeruginosa cell viability or morphology (Yakhnina, et al., 2015). AmiB (PA4947) in contrast to $E$. coli AmiB was found to be essential for $P$. aeruginosa survival (Yakhnina, et al., 2015). P. aeruginosa AmiB is required for cell separation during division, as depletion resulted in filamentous growth with a marked deficiency in the invagination of the inner membrane. Additionally, AmiB depletion decreased outer membrane impermeability for which the mechanism is unclear (Yakhnina, et al., 2015). P. aeruginosa AmiB requires the presence of additional activator dLytM proteins such as NIpD, NIpS, and EnvC (Yakhnina, et al., 2015).

P. aeruginosa also has two periplasmic amidases AmpDh2 (PA5485) and AmpDh3 (PA0807) that play a critical role in cell-wall recycling (Juan, et al., 2006). Both enzymes have significant homology with E. coli AmiD (Table 2). AmpDh2, akin to AmiD is anchored to the outer membrane (Uehara \& Park, 2007, Martinez-Caballero, et al., 2013). Both AmpDh2 and AmpDh3 process the peptide chain on the polymeric murein sacculus as well as 1, 6 anhydromuramyl peptides generated through the activities of lytic transglycosylases (Zhang, et al., 2013). The preferred substrate, however, for both these amidases is the murein sacculus (Zhang, et al., 2013). Comparing the activity between these two amidases, AmpDh3 has a higher specific activity on the murein sacculus (Lee, et al., 2013, Martinez-Caballero, et al., 2013, Zhang, et al., 2013). Crystal structures and catalytic activities of all the $P$. aeruginosa cell-wall recycling amidases have been reviewed elsewhere (Rivera, et al., 2016).

\section{Inner membrane}

The muropeptides that are generated by the periplasmic enzymes are transported into the cytoplasm via the inner membrane proteins which play an indispensable role in 
the recycling process. The inner membrane AmpG permease facilitates the diffusion of muropeptides from the periplasm into the cytoplasm (Park, 1993, Jacobs, et al., 1994) (Figure 1.2). The ampG gene was first identified in Enterobacter cloacae (Werner, et al., 1985). Since then, it has been found in other members of Enterobacteriaceae including C. freundii, Serratia marcescens, and Salmonella sp. The earliest indications that AmpG may be the primary permease in cell-wall recycling came from Normark's lab during the investigation of its role in $\beta$-lactamase expression (Lindquist, et al., 1993). Its role as a permease was confirmed when an ampG deletion resulted in the increased release of muropeptide in the media which was rescued upon complementation in trans (Jacobs, et al., 1994). The E. coli AmpG transports muropeptides that contain the disaccharide units GlcNAc-anhMurNAc across the inner membrane into the cytoplasm (Cheng \& Park, 2002). AmpG has 10 transmembrane helices, and ampG is the second ORF in a two-gene operon which may be post-transcriptionally regulated (Lindquist, et al., 1993, Chahboune, et al., 2005). Besides AmpG, E. coli also has another unique permease system, OppBCDF, which complexes with the muropeptide-binding protein MppA and plays a minor role in PG recycling (Goodell \& Higgins, 1987, Park, 1993) (Figure 1.4).

P. aeruginosa harbors two AmpG homologs, PA4393 (AmpG) and PA4218 (AmpP) (Table1.2) (Cheng, et al., 2000, Kong, et al., 2010). Both AmpG and AmpP are inner membrane permeases with 14 and 10 transmembrane helices, respectively (Kong, et al., 2010). The expression of $a m p G$ and $a m p P$ is regulated by the transcriptional regulator AmpR (PA4109) in an inducer (sub-MIC of $\beta$-lactam) dependent and independent manner, respectively (Balasubramanian, et al., 2012). Both ampG and ampP are the second genes in independent two-gene operons (Kong, et al., 2010). An uptake assay using fluorophore-conjugated muropeptides demonstrated that $P$. aeruginosa AmpG 
transports any muropeptides containing GlcNAc-1, 6 anhydroMurNAc (PerleyRobertson, et al., 2016). However, the in vivo role of both the permeases AmpG and AmpP in muropeptide transport remains to be elucidated. AmpG activity can be inhibited by the proton gradient uncoupler, carbonyl cyanide $m$-chlorophenyl hydrazine, suggesting that its activity is dependent on single proton motive force (Zhang, et al., 2010).

An alternative route of transfer of GlcNAc into the cytoplasm using NagE exists for both E. coli and $P$. aeruginosa (Plumbridge, 2009). The NagE phosphotransferase phosphorylates and imports GlcNAc into the cytoplasm, adding to the cytoplasmic pool of GlcNAc-6-P (Plumbridge, 2009). In E. coli, the absence of nagE resulted in a decrease of $50 \%$ of GlcNAc-6-P in the cytoplasm (Plumbridge, 2009). Whether this phosphotransferase plays a prominent role in cell-wall recycling in $P$. aeruginosa is yet to be explored. However, P. aeruginosa nagE (PA3761) mutants cannot grow using GlcNAc as the sole carbon source, suggesting a significant role for NagE in GlcNAc uptake (Korgaonkar \& Whiteley, 2011). In E. coli, the uptake and metabolism of MurNAc are mediated by a specific phosphotransferase MurP (Dahl, et al., 2004). This transporter is required for the growth of this bacterium in the presence of MurNAc as the sole carbon source (Dahl, et al., 2004). MurP has not been identified in P. aeruginosa.

Table 1. 2. List of genes involved in cell-wall recycling

\begin{tabular}{|c|c|c|c|c|c|}
\hline \multirow{2}{*}{ Proteins } & \multicolumn{2}{|c|}{ Genes } & \multicolumn{2}{|c|}{ Protein Homology (\%) } & \multirow{2}{*}{$\begin{array}{l}\text { Functional } \\
\text { Annotation }\end{array}$} \\
\hline & E. coli & $\begin{array}{c}P . \\
\text { aeruginosa }\end{array}$ & Identity $^{*}$ & Similarity $^{*}$ & \\
\hline \multicolumn{6}{|l|}{ Periplasm } \\
\hline PBP1a & $\begin{array}{l}\text { ponA } \\
m r c A\end{array}$ & PA5045 & 41 & 58 & $\begin{array}{l}\text { Transglycosylase } \\
\text { and transpeptidase }\end{array}$ \\
\hline PBP1b & $\begin{array}{l}\text { ponB/ } \\
m r c B\end{array}$ & PA4700 & 38 & 56 & $\begin{array}{l}\text { Transglycosylase } \\
\text { and transpeptidase }\end{array}$ \\
\hline
\end{tabular}




\begin{tabular}{|c|c|c|c|c|c|}
\hline PBP4 & $d a c B$ & PA3047 & 26 & 40 & $\begin{array}{l}\text { D-alanyl-D-alanine } \\
\text { carboxypeptidase } \\
\text { and endopeptidase }\end{array}$ \\
\hline PBP5 & $d a c A$ & PA3999 & 46 & 62 & $\begin{array}{l}\text { D-alanyl-D-alanine } \\
\text { carboxypeptidase }\end{array}$ \\
\hline PBP6 & dacC & PA3999 & 46 & 65 & $\begin{array}{l}\text { D-alanyl-D-alanine } \\
\text { carboxypeptidase }\end{array}$ \\
\hline PBP6b & $d a c D$ & NF & - & - & $\begin{array}{l}\text { D-alanyl-D-alanine } \\
\text { carboxypeptidase }\end{array}$ \\
\hline PBP7 & $p b p G$ & PA0869 & 63 & 75 & $\begin{array}{l}\text { D-alanyl-D-alanine } \\
\text { carboxypeptidase } \\
\text { and endopeptidase }\end{array}$ \\
\hline Slt70 & $\begin{array}{c}\text { slt } Y / s / t 7 \\
0\end{array}$ & PA3020 & 32 & 47 & $\begin{array}{l}\text { Lytic } \\
\text { transglycosylase }\end{array}$ \\
\hline MltA & $m / t A$ & PA1222 & 30 & 44 & $\begin{array}{l}\text { Lytic } \\
\text { transqlycosylase }\end{array}$ \\
\hline MltB & $m / t B$ & PA4444 & 66 & 78 & $\begin{array}{l}\text { Membrane-bound } \\
\text { lytic murein } \\
\text { transqlycosylase B }\end{array}$ \\
\hline SltB1 & $\mathrm{NF}$ & PA4001 & - & - & $\begin{array}{l}\text { Soluble lytic } \\
\text { transglycosylase B }\end{array}$ \\
\hline MltC & mltc & PA3020 & 13 & 20 & $\begin{array}{l}\text { Lytic } \\
\text { transglycosylase }\end{array}$ \\
\hline MltD & $m / t D$ & PA1812 & 33 & 50 & $\begin{array}{l}\text { Lytic } \\
\text { transglycosylase }\end{array}$ \\
\hline MltF & $m / t F$ & PA3764 & 33 & 50 & $\begin{array}{l}\text { Lytic } \\
\text { transglycosylase }\end{array}$ \\
\hline MltE & $m / t E$ & $\mathrm{NF}$ & - & - & $\begin{array}{l}\text { Lytic } \\
\text { transglycosylase }\end{array}$ \\
\hline RlpA & $r \mid p A$ & PA4000 & 27 & 40 & $\begin{array}{l}\text { Septal ring protein, } \\
\text { transglycolytic activity }\end{array}$ \\
\hline SltG & $\mathrm{NF}$ & PA1171 & - & - & $\begin{array}{l}\text { Lytic } \\
\text { transglycosylase }\end{array}$ \\
\hline MltF2 & $\mathrm{NF}$ & PA2865 & - & - & $\begin{array}{l}\text { Lytic } \\
\text { transglycosylase }\end{array}$ \\
\hline SltH & NF & PA3992 & - & - & $\begin{array}{l}\text { Lytic } \\
\text { transglycosylase }\end{array}$ \\
\hline AmiA & amiA & PA5538 & 28 & 40 & $\begin{array}{l}\mathrm{N} \text {-acetylmuramoyl-L- } \\
\text { alanine amidase }\end{array}$ \\
\hline AmiB & $a m i B$ & PA4947 & 34 & 47 & $\begin{array}{l}\mathrm{N} \text {-acetylmuramoyl-L- } \\
\text { alanine amidase }\end{array}$ \\
\hline AmiC & amic & PA5538 & 40 & 56 & $\begin{array}{l}\mathrm{N} \text {-acetylmuramoyl-L- } \\
\text { alanine amidase }\end{array}$ \\
\hline AmiC & amic & PA4947 & 35 & 47 & $\begin{array}{l}\mathrm{N} \text {-acetylmuramoyl-L- } \\
\text { alanine amidase }\end{array}$ \\
\hline AmiD & $a m i D$ & PA5485 & 41 & 56 & $\begin{array}{l}\mathrm{N} \text {-acetylmuramoyl-L- } \\
\text { alanine amidase } \\
\text { activity }\end{array}$ \\
\hline
\end{tabular}




\begin{tabular}{|c|c|c|c|c|c|}
\hline AmiD & $a m i D$ & PA0807 & 39 & 50 & $\begin{array}{l}\mathrm{N} \text {-acetylmuramoyl-L- } \\
\text { alanine amidase } \\
\text { activity }\end{array}$ \\
\hline $\mathrm{BgIX}$ & bgIX & PA1726 & 62 & 79 & Beta-glucosidase \\
\hline FlgJ & flgJ & PA1085 & 31 & 46 & $\begin{array}{l}\text { Flagellum-specific } \\
\text { glucosaminidase }\end{array}$ \\
\hline \multicolumn{6}{|c|}{ Inner membrane } \\
\hline AmpG & $a m p G$ & PA4393 & 22 & 35 & $\begin{array}{l}\text { Muropeptide } \\
\text { permease }\end{array}$ \\
\hline AmpG & $a m p P$ & PA4218 & 22 & 35 & $\begin{array}{l}\text { Putative muropeptide } \\
\text { permease }\end{array}$ \\
\hline OppB & oppB & PA4503 & 35 & 56 & $\begin{array}{l}\text { Muropeptide } \\
\text { transporter }\end{array}$ \\
\hline OppC & oppC & PA1808 & 24 & 41 & $\begin{array}{l}\text { Muropeptide } \\
\text { transporter }\end{array}$ \\
\hline OppC & oppC & PA2060 & 23 & 42 & $\begin{array}{l}\text { Muropeptide } \\
\text { transporter }\end{array}$ \\
\hline OppD & oppD & NF & - & - & $\begin{array}{l}\text { Muropeptide } \\
\text { transporter }\end{array}$ \\
\hline OppF & oppF & NF & - & - & $\begin{array}{l}\text { Muropeptide } \\
\text { transporter }\end{array}$ \\
\hline MppA & $m p p A$ & NF & - & - & $\begin{array}{l}\text { Periplasmic binding } \\
\text { component of } \\
\text { muropeptide- Opp } \\
\text { transporter }\end{array}$ \\
\hline MurP & murP & NF & - & - & $\begin{array}{l}\text { Anhydro-N- } \\
\text { acetylmuramic acid } \\
\text { permease }\end{array}$ \\
\hline NagE & nagE & PA3761 & 41 & 55 & $\begin{array}{l}\mathrm{N} \text {-acetylglucosamine } \\
\text { phosphotransferase }\end{array}$ \\
\hline \multicolumn{6}{|c|}{ Cytoplasm } \\
\hline NagZ & $n a g Z$ & PA3005 & 50 & 63 & $\begin{array}{l}\beta-N \text {-acetyl-D- } \\
\text { glucosaminidase }\end{array}$ \\
\hline AmpD & $a m p D$ & PA4522 & 57 & 69 & $\begin{array}{l}\mathrm{N} \text {-acetylmuramyl-L- } \\
\text { alanine amidase }\end{array}$ \\
\hline LdcA & $I d c A$ & PA5198 & 29 & 40 & $\begin{array}{l}\mathrm{L}, \mathrm{D}- \\
\text { carboxypeptidase }\end{array}$ \\
\hline AnmK & anmK & PA0666 & 49 & 64 & $\begin{array}{l}\text { Anhydro-N- } \\
\text { acetylmuramic acid } \\
\text { kinase }\end{array}$ \\
\hline MurQ & murQ & NF & - & - & $\begin{array}{l}\text { MurNAc-6-P } \\
\text { etherase }\end{array}$ \\
\hline AmgK & NF & PA0596 & - & - & MurNAc kinase \\
\hline MupP & NF & PA3172 & - & - & Phosphatase \\
\hline MurU & NF & PA0597 & - & - & $\begin{array}{l}\text { Nucleotidyltransferas } \\
\text { e }\end{array}$ \\
\hline $\operatorname{Nag} A$ & $\operatorname{nag} A$ & PA3758 & 30 & 47 & $\begin{array}{l}\text { GlcNAc and } \\
\text { anhMurNAc }\end{array}$ \\
\hline
\end{tabular}




\begin{tabular}{|c|c|c|c|c|l|}
\hline & & & & & deactylase \\
\hline $\mathrm{Mpl}$ & $\mathrm{mpl}$ & PA4020 & 59 & 72 & Murein peptide ligase \\
\hline $\mathrm{NagB}$ & $n a g B$ & $\mathrm{NF}$ & - & - & Deaminase \\
\hline
\end{tabular}

The data is compiled from EcoCyc and Pseudomonas Genome database website (Keseler, et al., 2009, Winsor, et al., 2016).

* The value for protein sequence identity and similarity of $E$. coli and $P$. aeruginosa was obtained using ClustalW2 "pairwise sequence alignments".

NF: Not Found

\section{Cytoplasm}

Once in the cytoplasm, the muropeptides are processed by various enzymes to form Lipid II composed of UDP-GIcNAcMurNac pentapeptide attached to a hydrophobic undecaprenol-pyrophosphate group (Mengin-Lecreulx, et al., 1991, Bouhss, et al., 2004). Lipid II is flipped across the cytoplasmic membrane into the periplasmic space, where GlcNAcMurNac pentapeptide is reincorporated into the growing cell-wall. There is an overlap between the enzymes mediating these reactions in $E$. coli and $P$. aeruginosa; however, certain enzymes remain unique to each bacterial system. These enzymes are detailed below.

\section{NagZ}

In the cytoplasm, the $\beta$ - $N$-acetylglucosaminidase NagZ processes the muropeptides (Yem \& Wu, 1976) (Figure1. 4). The enzymatic activity of NagZ was first identified in $E$. coli K-12 mutant strains which showed a deficiency in $\beta$ - $N$-acetylglucosaminidase (Yem $\& \mathrm{Wu}, 1976)$. The mutation was mapped to $y c f O$ that was renamed as nagZ following functional characterization of the protein (Yem \& Wu, 1976, Yem \& Wu, 1976, Hrebenda, 1979). NagZ cleaves the bond between GlcNAc and 1,6 anhydroMurNAc, leading to the 
formation of independent units of GlcNAc, and 1,6 anhMurNAc-peptide (Yem \& Wu, 1976). Deletion of the nagZ gene results in the accumulation of GlcNAc-anhMurNAc peptides (Yem \& Wu, 1976, Votsch \& Templin, 2000). Bioinformatics analyses of nagZ sequences led to its identification in numerous Gram-negative pathogens such as Klebsiella pneumonia, Vibrio cholera, P. aeruginosa, Haemophilus influenza, and Bordetella pertussis among others (Cheng, et al., 2000).

$P$. aeruginosa nagZ (PA3005) is predicted as the first gene in a two-gene operon along with PA3004, which encodes a phosphorylase. Similar to E. coli, NagZ (PA3005) was demonstrated to act as a $\beta-N$-acetylglucosaminidase (Stubbs, et al., 2008). Crystal structures of NagZ were solved along with its ligand. Using X-ray structures and molecular dynamics simulation, the enzymatic details of NagZ activity was elucidated (Acebron, et al., 2017). Most of the studies with NagZ (PA3005) have been done on its role in regulating antibiotic resistance which will be discussed in the following sections.

\section{AmpD and LdcA}

E. coli AmpD cleaves the peptide chain exclusively attached to the 1,6 anhydromuramyl moieties (Holtje, et al., 1994, Jacobs, et al., 1995). The first indication that this gene may play a critical role in cell-wall recycling stemmed from observations of its influence on $\beta$-lactamase induction (Tuomanen, et al., 1991). Its specific role as an amidase in recycling was confirmed when it was noticed that the loss of ampD resulted in the accumulation of GlcNAc-anhMurNAc with peptide chains (Holtje, et al., 1994, Jacobs, et al., 1995). Subsequently, a major increase of anhydromuramyl tripeptides in $a m p D$ deficient $E$. coli cells led to the identification of an LD-carboxypeptidase LdcA (Templin, et al., 1999). This enzyme removes the terminal D-alanine from the muramyl- 
tetrapeptides, thereby creating the tripeptides (Templin, et al., 1999). To this tripeptide, MurF attaches a dipeptide D-alanyl-D-alanine (D-Ala-D-Ala) forming a pentapeptide that participates in cross-linking (Uehara \& Park, 2007). The loss of $I d c A$ results in the formation of GlcNAc-MurNAc-tetrapeptides as the donor muropeptide, which is incapable of cross-linking as it does not contain the terminal D-Ala-D-Ala moiety. This deficient PG backbone eventually leads to cell lysis during the stationary phase of growth (Templin, et al., 1999).

P. aeruginosa also harbors an AmpD homolog (PA4522) (Table 1.2). The enzymatic activity of purified $P$. aeruginosa AmpD was demonstrated using synthetic muramyl and 1, 6 anhydromuramyl derivatives (Zhang, et al., 2013). AmpD activity was higher with the 1, 6 anhydromuropeptides as substrates as opposed to the muramyl derivatives (Zhang, et al., 2013, Rivera, et al., 2016). The cytoplasmic LD-carboxypeptidase in $P$. aeruginosa (PA5198) was also identified as a functional equivalent of E. coli LdcA (Korza \& Bochtler, 2005).

In the final steps of recycling processes, the PG sugars, GlcNAc, MurNAc, anhMurNAc, and the PG peptides L-Ala-y-D-Glu-meso-DAP-D-Ala-D-Ala rejoin the PG biosynthesis pathway in the cytoplasm.

\subsubsection{Convergence of recycling and biosynthesis}

The recovery of PG intermediates and its convergence into the biosynthesis pathway is a widely conserved process in bacteria and demonstrates their efficiency in preserving critical energy resources. The muropeptides generated in the cytoplasm through the activities of NagZ, AmpD, and LdcA rejoin the biosynthesis pathway through the 
sequential activity of AnmK, MurQ, NagA, and NagB in E. coli (White \& Pasternak, 1967, Calcagno, et al., 1984, Uehara, et al., 2005, Uehara, et al., 2006) (Figure 1.4).

In E. coli, AnmK kinase phosphorylates anhMurNAc to MurNAc-6-P which is processed by MurQ etherase to form GlcNAc-6-P (Uehara, et al., 2005). GlcNAc generated in the cytoplasm after the NagZ activity is phosphorylated to GlcNAc-6-P by NagK (Uehara \& Park, 2004). NagA deacetylates GlcNAc-6-P to GlcN-6-P (Park, 2001, Uehara, et al., 2006). GlcN-6-P undergoes deamination by NagB to form fructose-6phosphate, the initial substrate for glycolysis, PG and lipopolysaccharide synthesis (Calcagno, et al., 1984). GlcN-6-P can also be converted to GlcN $\alpha-1 \mathrm{P}$ by GlmM which is an intermediate in the PG synthesis pathway (Mengin-Lecreulx \& van Heijenoort, 1996) (Figure 1.4).

The set of enzymes for the reutilization of the PG sugars GlcNAc and MurNAc differ among $P$. aeruginosa and E. coli (Figure 1.4). In $P$. aeruginosa, an AnmK homolog PA0666 phosphorylates anhMurNAc to form MurNAc-6-P; subsequently, the phosphate is removed by MupP (PA3172) resulting in MurNAc (Bacik, et al., 2011, Borisova, et al., 2014, Borisova, et al., 2017, Fumeaux \& Bernhardt, 2017). MurNAc is further processed by two enzymes AmgK (PA0596) and MurU (PA0597) unique to Pseudomonads encoded in the same operon (Gisin, et al., 2013). AmgK (PA0596) converts MurNAc to MurNAc a-1P (Borisova, et al., 2014). A uridylyltransferase MurU (PA0597) acts on MurNAc $\alpha-1 P$ to form UDP-MurNAc (Gisin, et al., 2013). The presence of these two proteins bypasses the need for MurQ, NagA (PA3758), NagB, NagK, GlmM (PA4749) and GImS (PA5549) that are otherwise required for the synthesis of UDP-MurNAc in $E$. coli (Figure 1.4). This novel alternate pathway was identified in $P$. aeruginosa and $P$. 
putida (Gisin, et al., 2013, Borisova, et al., 2014). Homologs of AmgK and MurU have been identified in Proteobacteria but not in Enterobacteria (Borisova, et al., 2014).

AmpD amidase cleaves the peptide side chain from UDP-MurNAc resulting in the formation of free tri-, tetra-, and pentapeptides (L-Ala- - -D-Glu-meso-DAP-D-Ala-D-Ala) (Holtje, et al., 1994). LdcA carboxypeptidase cleaves the terminal D-alanine from a tetrapeptide resulting in tripeptides (Templin, et al., 1999). In E. coli, murein peptide ligase $(\mathrm{Mpl})$ recycles the tri-, tetra- and pentapeptides by ligating them to UDP-MurNAc formed in the PG biosynthesis pathway (Mengin-Lecreulx, et al., 1996, Herve, et al., 2007). Another enzyme, MurF can also ligate dipeptide D-Ala-D-Ala formed by an ATPdependent ligase Ddl, to a UDP-MurNAc tripeptide to form UDP-MurNAc pentapeptide (Duncan, et al., 1990, Zawadzke, et al., 1991, al-Bar, et al., 1992). Homologs of Mpl (PA4020) and MurF (PA4416) and Ddl (PA4201/PA4410) are found in P. aeruginosa; however, their role in cell-wall recycling has not been elucidated.

The tripeptide, L-Ala-y-D-Glu-m-DAP, can be broken down into individual amino acids. The E. coli MpaA amidase removes the meso-DAP from L-Ala-D-Glu (Uehara \& Park, 2003). E. coli YcjG epimerizes D-glutamate to L-glutamate forming L-Ala-L-Glu, which is hydrolyzed to individual amino acids by PepD (Schroeder, et al., 1994, Schmidt, et al., 2001). This pathway of amino acid degradation has not been studied in $P$. aeruginosa and bioinformatics analyses of the protein sequences of MpaA, YcjG, and PepD revealed no homologs in the $P$. aeruginosa PAO1 strain.

\subsection{Cell-wall recycling and antibiotic resistance}

Resistance is a distinctive quality of pathogens that have ensured the long tug of war between the discovery of novel antibiotics and bacterial survival. Antibiotics act at several checkpoints in the bacterial life cycle such as DNA replication, RNA, protein 
synthesis and activity as well as cell-wall biogenesis and recycling (Kohanski, et al., 2010). Being unique to bacteria, cell-wall synthesis and recycling are often the targets for many widely-used antibiotics such as $\beta$-lactams, fosfomycin, and glycopeptides (Bush, 2012). Consequently, the components of cell-wall recycling process play a key role in the development of resistance to the targeting antibiotics. Through a complex network of regulators, some of the cell-wall recycling components are also involved in the elaboration of cross-resistance to other classes of antibiotics such as fluoroquinolones, aminoglycosides, and macrolides (Kumari, et al., 2014). Here, we describe the role of cell-wall components in promoting antibiotic resistance in Gramnegative bacteria, focusing on $P$. aeruginosa.

\subsection{1 $\beta$-Lactam resistance involving Amp pathway}

$\beta$-lactams are the one of the earliest discovered and most commonly administered antibiotics worldwide. The $\beta$-lactams act by disrupting the cell-wall synthesis in bacteria (Tipper \& Strominger, 1965). Specifically, $\beta$-lactam antibiotics mimic the terminal D-AlaD-Ala residues of the amino acid side chains that serve as the substrates for the transpeptidase domains of HMM PBP's such as PBP1A, PBP1B, PBP2 and PBP3, locking them into an inactive state, thereby preventing PG cross-linking leading to cell lysis and death (Tipper \& Strominger, 1965, Zemelman, et al., 1987). Thus, it is not surprising that with the intracellular activation of $a m p C$, encoding a $\beta$-lactamase, the expression is tightly coordinated with the cell-wall recycling process in Gram-negative bacteria (Wiedemann, et al., 1998, Zeng \& Lin, 2013). The AmpR-AmpC system has been investigated thoroughly in Enterobacteriaceae. In many members of the Enterobacteriaceae, the induction of ampC expression in response to $\beta$-lactam 
antibiotics is triggered through the activation of a transcriptional regulator AmpR by the cell-wall degradation products (Lindberg \& Normark, 1987, Lindquist, et al., 1989, Tolg, et al., 1993, Wiedemann, et al., 1998, Dik, et al., 2017). The induction of ampC by AmpR also requires the products of $a m p G, a m p D$, ampDh2, $a m p D h 3$, and nagZ, all of which are involved in PG recycling, as described in previous sections. Studies from the Mathee lab suggest that the AmpR-AmpC (PA4109-PA4110) system might operate differently in P. aeruginosa (Balasubramanian, et al., 2015). Expression of the genes ampG, ampP, $a m p D, a m p D h 2, a m p D h 3$, and nag $Z$ in $P$. aeruginosa are differentially regulated by AmpR in the presence and absence of $\beta$-lactam (Balasubramanian, et al., 2012). AmpG is the permease that transports PG degradation products from the periplasm to cytoplasm. In Enterobacteriaceae members, an ampG mutant is unable to recycle PG and loses the ability to induce ampC expression (Korfmann \& Sanders, 1989, Jacobs, et al., 1994) (Table 1.3). Inactivation of ampG can restore susceptibility to $\beta$-lactams even in pan-resistant $P$. aeruginosa clinical isolates (Zamorano, et al., 2011). $P$. aeruginosa also has an additional AmpG homolog known as AmpP (PA4218), both of which are presumed to be involved in $a m p C$ induction (Kong, et al., 2010, Zhang, et al., 2010).

The AmpD amidase was identified as being important for $\beta$-lactamase induction in $C$. freundii and Enterobacter cloacae (Lindquist, et al., 1989). The absence of ampD leads to the accumulation of anhMurNAc-tripeptide in the cytosol and constitutive overproduction of $\beta$-lactamase even in the absence of induction, resulting in a high resistance to $\beta$-lactams (Jacobs, et al., 1995). The most common mechanism for constitutive ampC overexpression in clinical strains leading to $\beta$-lactam resistance in Enterobacteriaceae, as well as $P$. aeruginosa, is to mutate ampD (Langaee, et al., 2000, Juan, et al., 2005, Kaneko, et al., 2005, Babouee Flury, et al., 2016) (Table 1.3). P. aeruginosa has three different AmpD homologs, AmpD (PA4522), AmpDh2 (PA5485), 
and AmpDh3 (PA0807) that are responsible for de-repression of ampC expression in a step-wise manner (Juan, et al., 2006, Schmidtke \& Hanson, 2008). Deletion of all three $a m p D$ genes results in increased resistance due to complete de-repression of $a m p C$ expression resulting in very high MIC for the $\beta$-lactams drugs including cephalosporins and monobactams (Juan, et al., 2006, Moya, et al., 2008).

NagZ is a $\beta$ - $N$-acetylglucosaminidase that removes GlcNAc to generate the 1,6 anhydromuropeptides, the putative activators of $\mathrm{AmpR}$ that are required for the induction of ampC (Asgarali, et al., 2009). Mutations in nagZ result in low levels of ampC expression that enhances the susceptibility to $\beta$-lactam antibiotics (Asgarali, et al., 2009, Zamorano, et al., 2010). Deletion of nagZ also reversed the resistance due to $a m p C$ hyper-expression in $a m p D$ and $d a c B$ mutants (Zamorano, et al., 2010). Based upon these findings, to increase the bacterial susceptibility to $\beta$-lactams, small molecule inhibitors gluco-nagstatin, LOGNAc, and PUGNAc which are analogous to the NagZ substrates that have oxocarbenium-like transition states have been synthesized (Figure 1.5) (Stubbs, et al., 2007, Mondon, et al., 2013). Among the three, PUGNAc was the most potent inhibitor of $\beta$ - $\mathrm{N}$-acetylglucosaminidase (Horsch, et al., 1991). However, all three were found to inhibit human glucosaminidases rendering them less useful (Stubbs, et al., 2007). 


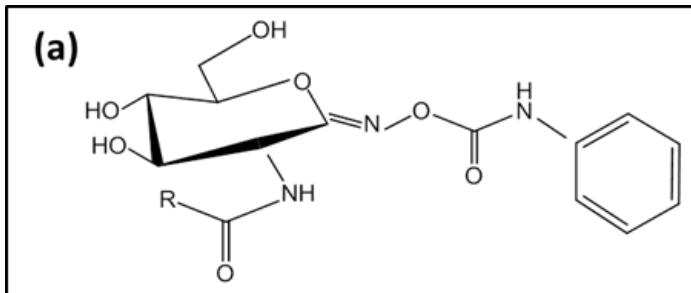

PUGNAC

(c)

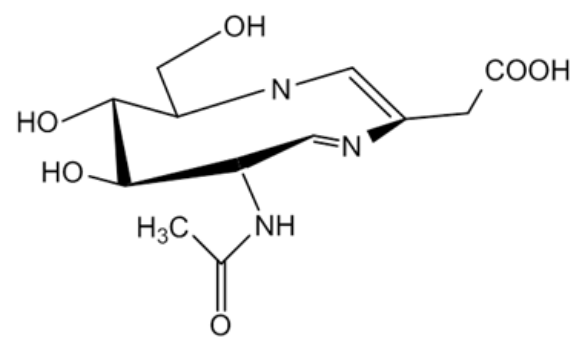

gluco-Nagstatin (b)

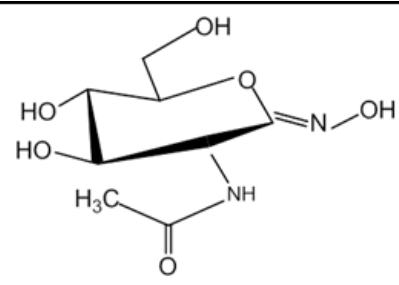

LOGNAC

(d)

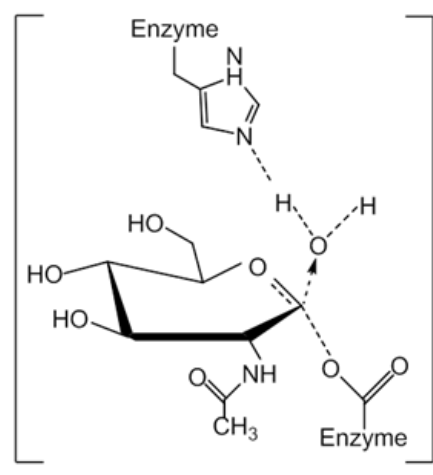

NagZ substrate

Figure 1.5. Small molecule inhibitors of $\mathrm{N}$-acetyl- $\beta$-glucosaminidase NagZ. (a) $\mathrm{O}$ (2-Acetamido-2-deoxy-D-gucopyranosylidene) amino N-phenylcarbamate (PUGNAc) (b) 1, N-acetylglucosaminono-1,5-lactone oxime (LOGNAc) (c) gluco analogue of 8acetamido-5,6,7,8-tetrahydro-6,7-dihydroxy-5-(hydroxymethyl)imidazo[1,2-a] pyridine-2acetic acid nagstatin (gluco-Nagstatin) (d) Transition state of NagZ substrate analogous to the small molecule inhibitors (Horsch, et al., 1991, Stubbs, et al., 2007).

Table 1.3. MIC of mutant strains of $P$. aeruginosa

\begin{tabular}{|c|c|c|c|c|c|c|c|c|c|c|}
\hline \multirow{2}{*}{ Strains } & \multicolumn{9}{|c|}{ MIC $(\mu \mathrm{g} / \mathrm{mL})$} & \multirow{2}{*}{ References } \\
\hline & AMP & PIP & CTX & CAZ & CEF & FOX & ATM & IMI & MER & \\
\hline \multirow{10}{*}{$\begin{array}{l}\text { PAO1 } \\
\text { dacB } \\
\text { dacC } \\
p b p G^{*} \\
a m p C^{*} \\
\text { dacB } \\
a m p C^{*} \\
\text { dacC } \\
\text { ampC } \\
p b p G \\
\text { ampC } \\
\text { dacB } \\
\text { dacC } \\
\text { dacB }\end{array}$} & 1024 & 3.2 & 7 & 1.4 & 1.53 & 1024 & 10.4 & 1.2 & 0.41 & \multirow{10}{*}{$\begin{array}{l}\text { (Ropy, et } \\
\text { al., 2015) }\end{array}$} \\
\hline & 1024 & 16 & 256 & 8 & 4 & 1024 & 8 & 1 & 0.5 & \\
\hline & 1536 & 4 & 8 & 1 & 0.5 & 1024 & 2 & 0.5 & 0.5 & \\
\hline & 512 & 4 & 16 & 1 & 1 & 1024 & 4 & 1 & 0.5 & \\
\hline & 32 & 2 & 8 & 1 & 1 & 64 & 4 & 0.12 & 0.25 & \\
\hline & 32 & 2 & 8 & 1 & 0.5 & 96 & 4 & 0.12 & 0.25 & \\
\hline & 16 & 2 & 4 & 0.75 & 0.5 & 64 & 2 & 0.06 & 0.25 & \\
\hline & 32 & 4 & 8 & 1 & 0.5 & 96 & 3 & 0.12 & 0.25 & \\
\hline & 1024 & 128 & 512 & 16 & 4 & 1024 & 16 & 0.5 & 0.5 & \\
\hline & 16 & 2 & 4 & 1 & 0.5 & 64 & 2 & 0.06 & 0.12 & \\
\hline
\end{tabular}




\begin{tabular}{|c|c|c|c|c|c|c|c|c|c|c|}
\hline $\begin{array}{l}\text { dacC } \\
\text { ampC* }\end{array}$ & & & & & & & & & & \\
\hline $\begin{array}{l}\text { dacB } \\
p b p G^{*}\end{array}$ & 2048 & 16 & 256 & 8 & 4 & 1024 & 8 & 0.5 & 0.25 & \\
\hline $\begin{array}{l}\text { dacB } \\
p b p G \\
a m p C^{*}\end{array}$ & 32 & 2 & 6 & 0.75 & 0.5 & 64 & 2 & 0.12 & 0.5 & \\
\hline $\begin{array}{l}\text { dacC } \\
p b p G^{*}\end{array}$ & 1024 & 6 & 8 & 1 & 0.5 & 1024 & 2 & 0.5 & 0.25 & \\
\hline $\begin{array}{l}\text { dacC } \\
p b p G \\
a m p C^{*}\end{array}$ & 24 & 3 & 4 & 0.75 & 0.5 & 64 & 3 & 0.06 & 0.25 & \\
\hline sltB1^ & nd & 12 & 16 & 1 & nd & nd & nd & 0.75 & nd & \multirow{9}{*}{$\begin{array}{l}\text { (Cavallari, } \\
\text { et al., 2013) }\end{array}$} \\
\hline$m / t B^{\wedge}$ & nd & 8 & 12 & 1.5 & nd & nd & nd & 1.5 & nd & \\
\hline$s / t^{\wedge}$ & nd & 1.5 & 6 & 0.19 & nd & nd & nd & 1 & nd & \\
\hline$m l t F^{\wedge}$ & nd & 2 & 6 & 0.38 & nd & nd & nd & 1 & nd & \\
\hline $\begin{array}{l}\text { sltB1 } \\
a m p C^{\wedge}\end{array}$ & nd & 1.5 & 2 & 0.5 & nd & nd & nd & 0.25 & nd & \\
\hline $\begin{array}{l}m l t B \\
a m p C^{\wedge}\end{array}$ & nd & 6 & 8 & 1 & nd & nd & nd & 0.38 & nd & \\
\hline $\begin{array}{l}\text { sltB1 } \\
\text { dacB }\end{array}$ & nd & $\begin{array}{c}>25 \\
6\end{array}$ & $>256$ & 24 & nd & nd & nd & 1 & nd & \\
\hline $\begin{array}{l}m / t B \\
\text { dacB }\end{array}$ & nd & 192 & $>256$ & 16 & nd & nd & nd & 1.5 & nd & \\
\hline $\begin{array}{l}m / t B \\
\text { sltB1^ }\end{array}$ & nd & 24 & 64 & 2 & nd & nd & nd & 1 & nd & \\
\hline$a m p G^{*}$ & 32 & nd & 8 & 1 & nd & nd & nd & nd & nd & $\begin{array}{c}\text { (Zhang, et } \\
\text { al., 2010) }\end{array}$ \\
\hline$a m p D^{\wedge}$ & nd & nd & nd & 8 & 4 & nd & 6 & 2 & 1 & \multirow{3}{*}{$\begin{array}{c}\text { (Juan, et al., } \\
\text { 2006) }\end{array}$} \\
\hline$\underset{\wedge}{a m p D h 2}$ & nd & nd & nd & 0.75 & 1 & nd & 1 & 1 & 0.38 & \\
\hline$\underset{\wedge}{a m p D h 3}$ & nd & nd & nd & 1 & 1 & nd & nd & 1 & 0.38 & \\
\hline$a m p R^{\wedge}$ & 12 & 1.5 & nd & 1 & 1 & nd & 0.75 & 0.38 & 1.5 & $\begin{array}{c}\text { (Balasubra } \\
\text { manian, et } \\
\text { al., 2012) }\end{array}$ \\
\hline$p o x A^{\wedge}$ & 256 & 4 & 6 & 1.5 & 1.25 & nd & 2 & 1.25 & 0.44 & \multirow{3}{*}{$\begin{array}{l}\text { (Zincke, et } \\
\text { al., 2015) }\end{array}$} \\
\hline$p o x B^{\wedge}$ & 256 & 4 & 6 & 1.5 & 1 & nd & 2 & 1.5 & 0.38 & \\
\hline $\begin{array}{l}\text { ampC } \\
p^{\prime} \times B^{\wedge}\end{array}$ & 24 & 4 & 6 & 1.5 & 1 & nd & 3 & 0.44 & $\begin{array}{c}0.31 \\
5 \\
\end{array}$ & \\
\hline nag $Z^{\wedge}$ & nd & nd & nd & 1 & 1.5 & nd & 4 & 1 & 0.38 & \multirow{4}{*}{$\begin{array}{l}\text { (Zamorano, } \\
\text { et al., 2011) }\end{array}$} \\
\hline creD* & nd & nd & nd & 8 & 4 & nd & 12 & 2 & 1 & \\
\hline $\begin{array}{l}\text { creD } \\
\text { nagZ* }\end{array}$ & nd & nd & nd & 2 & 1.5 & nd & 4 & 1 & 0.38 & \\
\hline dacB & nd & nd & nd & 1.5 & 2 & nd & 4 & 1 & 0.38 & \\
\hline
\end{tabular}




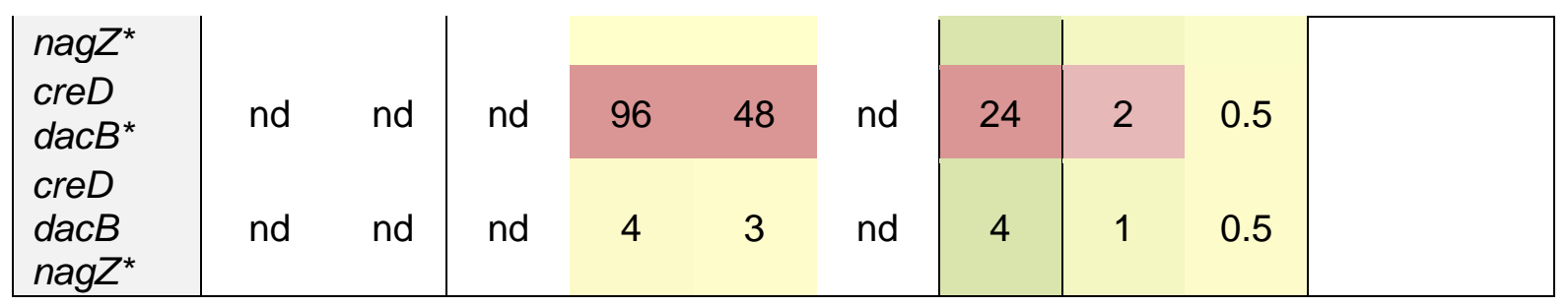

Table 1. 3. MIC levels of PAO1 and the corresponding deletion strains are indicated in the left. The MIC values of PAO1, shown in yellow, is an average of values as found in the cited references. Any values greater than PAO1 are shown in gradation of orangered. Values lower than PAO1 shown in gradations of yellow- green.

AMP, Ampicillin; PIP, Piperacillin; CTX, Cefotaxime; CAZ, Ceftazidime; CEF, Cefepime; FOX, Cefoxitin; ATM, Aztreonam; IMI, Imipenem; MER, Meropenem.

${ }^{*} \mathrm{MIC}$ determined by broth microdilution

${ }^{\wedge} \mathrm{MIC}$ determined by E-test

nd: not determined.

Details of $P G$ recycling and their ties with $\beta$-lactam resistance have been studied in Enterobacteriaceae members. The role of many orthologous proteins including AmpR, AmpC, AmpD, and AmpG have been demonstrated in $P$. aeruginosa and appear to have similar roles to their Enterobacteriaceae counterparts (Langaee, et al., 2000, Kong, et al., 2010, Johnson, et al., 2013). In addition, new players such as ampP, ampDh2, and ampDh3 have been identified in $P$. aeruginosa (Juan, et al., 2006, Kong, et al., 2010). Moreover, the localization of $P$. aeruginosa AmpR as an inner membrane protein with the helix-turn-helix domain in the cytoplasm and effector-binding domain in the periplasm leads to unique questions as to how the effector molecules are transported to their binding sites on AmpR (Caille, et al., 2014).

\subsection{2 $\beta$-Lactam resistance through other cell-wall-related genes}

Other members involved in cell-wall recycling, namely, the LTs and the LMM PBPs also modulate $\beta$-lactam resistance. The periplasmic LTs are the major PG degradative enzymes that generate 1, 6-anhydro-MurNAc muropeptides (Lee, et al., 2013). In E. coli, a double deletion of LT encoding genes $m I t A-m / t B$, slt70-mltA or slt70-mltB and a triple 
deletion mltA-m/tB-slt70 exhibit a higher loss in $\beta$-lactamase activities than any one single deletion that correlates with the increasing decline in murein turnover (Kraft, et al., 1999). In P. aeruginosa, the loss of SIt and MltF is associated with a decrease in $\beta$ lactam resistance (Cavallari, et al., 2013). However, the loss of SltB1 and MltB led to an AmpC-independent increase in resistance to $\beta$-lactams, piperacillin, and cefotaxime (Cavallari, et al., 2013, Lamers, et al., 2015). The loss of the other P. aeruginosa LTs' MltA, MltD, MltF2, SltG, and SltH did not change the resistance profile (Cavallari, et al., 2013)

The LMM PBPs comprise a group of enzymes that have endopeptidase and carboxypeptidase activity (Sauvage, et al., 2008). Three of the five LMM PBPs (PBP4, PBP5, PBP6, PBP6b, PBP7/8) were investigated for their role in $\beta$-lactam resistance in E. coli (Sarkar, et al., 2010, Sarkar, et al., 2011). Loss of PBP5 reduces the MIC for penicillins and cephalosporins whereas PBP6 and PBP6b removal have no resistance phenotype (Sarkar, et al., 2010). However, the lower MIC in PBP5 mutants can be partially restored by PBP6B. This suggests that PBP5 is important for $\beta$-lactam resistance followed by PBP6b (Sarkar, et al., 2011). However, the role of PBP4, PBP6, and PBP7/8 in antibiotic resistance is not well understood.

P. aeruginosa has three LMM PBPs (PBP4, PBP5, and PBP7) whose role in $\beta$ lactam resistance has been investigated (Moya, et al., 2009, Ropy, et al., 2015). Clinical mutants of PBP4 (PA3047) are associated with increased $\beta$-lactam resistance (Moya, et al., 2009). In fact, a mutation in PBP4 leads to a one step upregulation of ampC resulting in clinical $\beta$-lactam resistance (Moya, et al., 2009). Similar to PBP6b in E. coli, the role of PBP5 is not evident, unless in the PBP4 background where the double mutant further increases $\beta$-lactam resistance (Table 3). 
Interestingly, the PBPs in E. coli and $P$. aeruginosa play contradicting roles in $\beta$ lactam resistance. This may be reflective of the different mechanism of resistance, as $E$. coli has a non-inducible ampC (Honore, et al., 1989). One speculation is that $E$. coli PBP5 could act as a "trap" for $\beta$-lactams thereby preventing access to their targets, the HMM PBPs whereas in $P$. aeruginosa loss of PBP4 results in increased production of the AmpR effector which escalates ampC $\beta$-lactamase expression and thereby the MIC (Moya, et al., 2009, Sarkar, et al., 2010, Moya, et al., 2012, Lee, et al., 2015).

\subsubsection{Indirect regulation of $\beta$-lactam resistance through AmpR}

Although $P$. aeruginosa AmpR acts as the nexus for AmpC $\beta$-lactamase induction and cell-wall recycling processes, it also modulates the activity of other proteins that regulate $\beta$-lactam resistance. One of these is the second chromosomally encoded $\beta$ lactamase, PoxB (PA5514) in P. aeruginosa (Kong, et al., 2005). PoxB is a carbapenemase, which affects $\beta$-lactam resistance, in the absence of the major $\beta$ lactamase AmpC and outer membrane porin OprD (Zincke, et al., 2015). Although no

direct relation is seen between cell-wall recycling and poxB, AmpR is found to be a negative regulator of poxB through a yet unknown mechanism (Kong, et al., 2005, Zincke, et al., 2015).

Another mechanism of $\beta$-lactam resistance is through expulsion via efflux pumps namely MexAB-OprM (Poole, et al., 1993). Expression of mexAB is repressed by a regulator MexR (Poole, et al., 1996). AmpR is required for mexR expression, suggesting an opposite impact on $\beta$-lactam resistance (Balasubramanian, et al., 2012). Moreover, deleting $a m p C$ and or ampR in PAO1 abolishes $\beta$-lactam resistance despite having a functional MexAB system or PoxB, suggesting that the AmpC is the major determinant of 
$\beta$-lactam resistance (Balasubramanian, et al., 2012, Kumari, et al., 2014, Zincke, et al., 2015).

Another mechanism of $\beta$-lactamase induction is mediated through the twocomponent system CreBC and its effector, an inner membrane protein CreD (Moya, et al., 2009). In E. coli, CreB has been identified as a transcriptional factor that differentially regulates up to eight genes under diverse nutritional conditions (Avison, et al., 2001). E. coli $\mathrm{CreB}$ was also found to regulate the expression of Aeromonas hydrophila $\beta$ lactamase (Avison, et al., 2004). This two-component system in P. aeruginosa plays a pivotal role in $\beta$-lactam resistance and bacterial fitness (Zamorano, et al., 2010, Zamorano, et al., 2014). The inactivation of dacB (PBP4) leads to an increased expression of $c r e B C, c r e D$, and $a m p C$. The increased expression of $a m p C$ is accompanied by elevated MIC of $\beta$-lactams, which reverts to wild-type levels upon deletion of creBC (Zamorano, et al., 2014). The association of $c r e B C$ and $\beta$-lactam resistance was seen only in $d a c B$ mutants and not with other mutations that confer high resistance namely, ampD, ampDh2 and ampDh3 (Moya, et al., 2009). Moreover, these resistance phenotypes regulated through $c r e B C$ were lost in the absence of NagZ and AmpG (Moya, et al., 2009, Zamorano, et al., 2010, Zamorano, et al., 2014) (Table 3). Although the interplay of the cell-wall recycling components, $a m p C$ expression, and the cre $B C$-cre $D$ system is found to regulate $\beta$-lactam resistance, the underlying details remain to be elucidated.

\subsubsection{Cell-wall components contributing to non- $\beta$-lactam resistance}

In addition to the various mechanisms by which the different components involved in

PG biosynthesis and recycling confer $\beta$-lactam resistance, some cell-wall components 
acting through AmpR also play a role in resistance to non- $\beta$-lactam antibiotics (Kumari, et al., 2014). Some specific examples are discussed below.

\section{Fluoroquinolone resistance}

Quinolones, specifically fluoroquinolones, are potent, broad-spectrum synthetic antibiotics that inhibit DNA replication by targeting bacterial DNA gyrase (Gramnegatives) or topoisomerase II (Gram-positives) (Drlica, et al., 2008). Due to their effectiveness against both Gram-positive and Gram-negative bacteria, extensive use of fluoroquinolones has led to a rapid rise in resistance (Chatterjee, et al., 2016, Dan, et al., 2016, Bader, et al., 2017). Development of resistance to quinolones entails multiple mechanisms (Hernandez, et al., 2011, Hooper \& Jacoby, 2015). The primary mechanism of quinolone resistance in Gram-negative bacteria is through mutations in target genes gyrA and gyrB (Yamagishi, et al., 1981, Yamagishi, et al., 1986, Nakamura, et al., 1989, Drlica \& Zhao, 1997). Overexpression of MDR efflux pumps can also add significantly to the quinolone resistance especially in the presence of target mutations (Poole, 2000). Unexpectedly for synthetic antibiotics, horizontal gene transfer also plays role in quinolone resistance. A plasmid-mediated resistance gene qnr encoding a pentapeptide repeat protein protects the bacterial topoisomerases (gyrase and topoisomerase IV) from quinolone activity (Tran \& Jacoby, 2002, Robicsek, et al., 2006).

In $P$. aeruginosa, the MexEF-OprN system is involved in efflux of fluoroquinolones, chloramphenicol, and trimethoprim (Kohler, et al., 1997). This efflux system is under the control of three different regulators; the positive regulator MexT, and the negative regulators MexS and MvaT (Kohler, et al., 1999, Westfall, et al., 2006, Uwate, et al., 2013). P. aeruginosa AmpR negatively regulates the expression of the mexEF-oprN 
operon and MexT (Balasubramanian, et al., 2012). Consequently, PAO $\Delta a m p R$ mutants are more resistant to fluoroquinolones compared to the wild type (Balasubramanian, et al., 2012). Repression of the mexEF operon by AmpR is $\beta$-lactam independent (Balasubramanian, et al., 2012), suggesting that AmpR functions diversified from the cell-wall synthesis pathway. It is important to note that AmpR positively and negatively regulates resistance to $\beta$-lactams and quinolones, respectively, highlighting the intricate and complex balance of regulation of antibiotic resistance (Balasubramanian, et al., 2012, Balasubramanian, et al., 2015).

\section{Aminoglycosides resistance}

Traditionally, the aminoglycoside class of antibiotics is used to treat Gram-negative infections. They bind to the $16 \mathrm{~S}$ ribosomal subunit causing it to mistranslate leading to the synthesis of aberrant proteins (Davies, et al., 1964, Kotra, et al., 2000). Aminoglycosides, such as tobramycin, along with $\beta$-lactams form the first line of $P$. aeruginosa treatment for cystic fibrosis patients (Waters \& Smyth, 2015). One of the primary determinants of aminoglycoside resistance in $P$. aeruginosa and other Gramnegative pathogens are the presence of active efflux pumps (Moore, et al., 1999, Morita, et al., 2012). The upregulation of the MexXY efflux pump in $P$. aeruginosa is considered the most common mechanism of aminoglycoside resistance (Poole, 2011). Aminoglycoside resistance can also be conferred by drug modifying enzymes such as aminoglycoside acetyl-, nucleotidyl-, and phospho-transferases (Lovering, et al., 1987, Jacoby, et al., 1990, Shaw, et al., 1993, Azucena \& Mobashery, 2001, Ramirez \& Tolmasky, 2010). Mutations in aac, aphA1, and aadB that encode the aminoglycoside 
modifying genes have been identified in clinical $P$. aeruginosa isolates (Kitao, et al., 2009, Coyne, et al., 2010, Teixeira, et al., 2016).

In the presence of $\beta$-lactams, $P$. aeruginosa AmpR negatively regulates the expression of mexX, mexY, and aph; the latter encodes aminoglycoside phosphotransferse (Kumari, et al., 2014). However, this negative regulation is not translated into an observable phenotype. On the contrary, loss of ampR increases aminoglycoside (amikacin and tobramycin) susceptibility in $P$. aeruginosa suggesting a positive regulation (Kumari, et al., 2014). Thus, even with an increased expression of efflux and drug-modifying proteins, the $a m p R$ mutants are more susceptible to aminoglycosides, suggesting the existence of post- translational modifications in this complex regulatory network.

\subsection{Dissertation overview}

Pseudomonas aeruginosa and its various aspects of pathogenicity including antibiotic resistance has been the focus of researchers for many years. Previously our lab has explored the transcriptional factor $\mathrm{AmpR}$ that is known to regulate $\mathrm{AmpC}$ expression. Its role in $P$. aeruginosa was found to be more diverse and far-reaching when compared to that in other Gram-negatives such as E. coli and C. freundii (Balasubramanian, et al., 2015). However, similar to what is found in C. freundii and $E$. cloacae, it is that $P$. aeruginosa AmpR binds with an activating effector generated during cell-wall recycling under $\beta$-lactam stress, which results in induction of AmpC (Vadlamani, et al., 2015, Dik, et al., 2017). The identity of this effector had not been previously established in $P$. aeruginosa. Also, cell-wall recycling has predominantly been studied only in E. coli. 
In this dissertation, we investigate cell-wall recycling in $P$. aeruginosa. The structures of cell-wall recycling products or muropeptides found in $P$. aeruginosa are described as well as a method to quantitate muropeptides (Chapter 2). Additionally, we were able to identify the AmpR effector that is generated under $\beta$-lactam induction and binds to AmpR (Chapter 2). Futhermore, the role of permeases PA4393 and PA4218 in cell-wall recycling and antibiotic resistance are also investigated (Chapter 3). We also investigate a putative periplasmic $\mathrm{N}$-acetylglucosaminidase that may play a role in generating the periplasmic effectors of AmpR (Chapter 4). Finally, the localization of AmpR is investigated and given its extensive regulon in $P$. aeruginosa, it is developed as an antibacterial target (Chapter 5). A novel phenotypic screening assay that identifies inhibitors of AmpR is discussed (Chapter 5). 
Chapter 2

Muropeptides in Pseudomonas aeruginosa and their role as elicitors of $\beta$-Lactam antibiotic resistance

This chapter has been published:

Mijoon Lee ${ }^{+}$, Supurna Dhar ${ }^{+}$, Stefania De Benedetti, Dusan Hesek, Bill Boggess, Blas

Blzquez, Kalai Mathee, and Shahriar Mobashery

Angew. Chem. Int. Ed. 2016, 55, 1 - 6

${ }^{+}$Authors contributed equally 


\subsection{Overview}

This chapter is reproduced verbatim from the manuscript published in Angewandte Chemie International Edition. The supplementary portion of the manuscript has been integrated in the main text. I performed the murpeptide isolation from wholecell and fractions, the induction experiments with $P$. aeruginosa PAO1 strains, and the colony forming unit for muropeptide quantitation. The synthesis of muropeptide standards was perfomed by Dr. Mijoon Lee and Dr. Dusan Hesek. The LC/MS analysis was performed by Dr. Mijoon Lee and Dr. Bill Bogess. The induction with mutant strains of $P$. aeruginosa was performed by Dr. Blas Blazquez and Dr. Stefania DeBenetti.

\subsection{Abstract}

Muropeptides are a group of bacterial natural products generated from the cell wall in the course of its turnover. These compounds are cell-wall recycling intermediates and are also involved in signaling within the bacterium. However, the identity of these signaling molecules remains elusive. The identification and characterization of 20 muropeptides from Pseudomonas aeruginosa is described. The least abundant of these metabolites is present at 100 and the most abundant at 55,000 molecules per bacterium. Analysis of these muropeptides under conditions of induction of resistance to a b-lactam antibiotic identified two signaling muropeptides ( $\mathrm{N}$-acetylglucosamine-1,6-anhydro- $\mathrm{N}$ acetylmuramyl pentapeptide and 1,6-anhydro-N-acetylmuramyl pentapeptide). Authentic synthetic samples of these metabolites were shown to activate expression of blactamase in the absence of any $\beta$ lactam antibiotic, thus indicating that they serve as chemical signals in this complex biochemical pathway.

Keywords: antibiotic resistance; bacteria; muropeptides; peptidoglycan; $\beta$-lactamase 


\subsection{Introduction}

The cell wall (also known as the sacculus) is a complex macromolecular polymer that encases the bacterium. Its major constituent is comprised of repeating $\mathrm{N}$ acetylglucosamine (NAG)-N-acetylmuramic acid (NAM), with a pentapeptide stem attached to the NAM unit (Vollmer, et al., 2008, J. F. Fisher, 2015). Cell wall is critical for survival of the bacterium, hence, the cell wall and its biosynthetic machinery are targets of antibiotics (Bush, 2013). Cell wall is synthesized by polymerization of Lipid II, (van Heijenoort, 2007, Qiao, et al., 2014) resulting in the NAG-NAM backbone, which is subsequently crosslinked to the neighboring strand through the peptide stem (Figure 2.1). In parallel, degradative processes that turn over segments of the assembled cell wall also take place (van Heijenoort, 2007, Park \& Uehara, 2008). An important event in this turnover is mediated by a family of enzymes called lytic transglycosylases (LTs), whose reactions generate a series of natural products referred to collectively as muropeptides.

The reactions of all eight Escherichia coli LTs have been studied in vitro (Scheurwater, et al., 2008, van Heijenoort, 2011, Lee, et al., 2013, Yunck, et al., 2016).These enzymes generate a transient oxocarbenium species (1) at the muramyl moiety of the peptidoglycan, which results in the cleavage of the $\beta-1,4$-glycosidic bond between a NAM and a NAG (Figure 2.1), giving rise to the 1,6-anhydromuramyl moiety (2 and 3). Some LTs perform this reaction at the ends of the peptidoglycan, the so called exolytic reaction, giving rise to the NAG-anhNAM disaccharides (2). Others perform the reaction in the middle of the peptidoglycan, the endolytic reaction, which gives rise to a longer backbone for the sugar (3). These released muropeptides are translocated to the cytoplasm by the permease AmpG (and possibly by AmpP in Pseudomonas aeruginosa) 
(Kong, et al., 2010). Once in the cytoplasm, the muropeptides enter the cell-wall recycling process, regenerating Lipid II (van Heijenoort, 2007, van Heijenoort, 2011, Johnson, et al., 2013). Alternatively, other muropeptides are involved in signaling functions, leading to disparate responses such as antibiotic resistance, virulence, and inflammation (Boudreau, et al., 2012, Fisher \& Mobashery, 2014, Balasubramanian, et

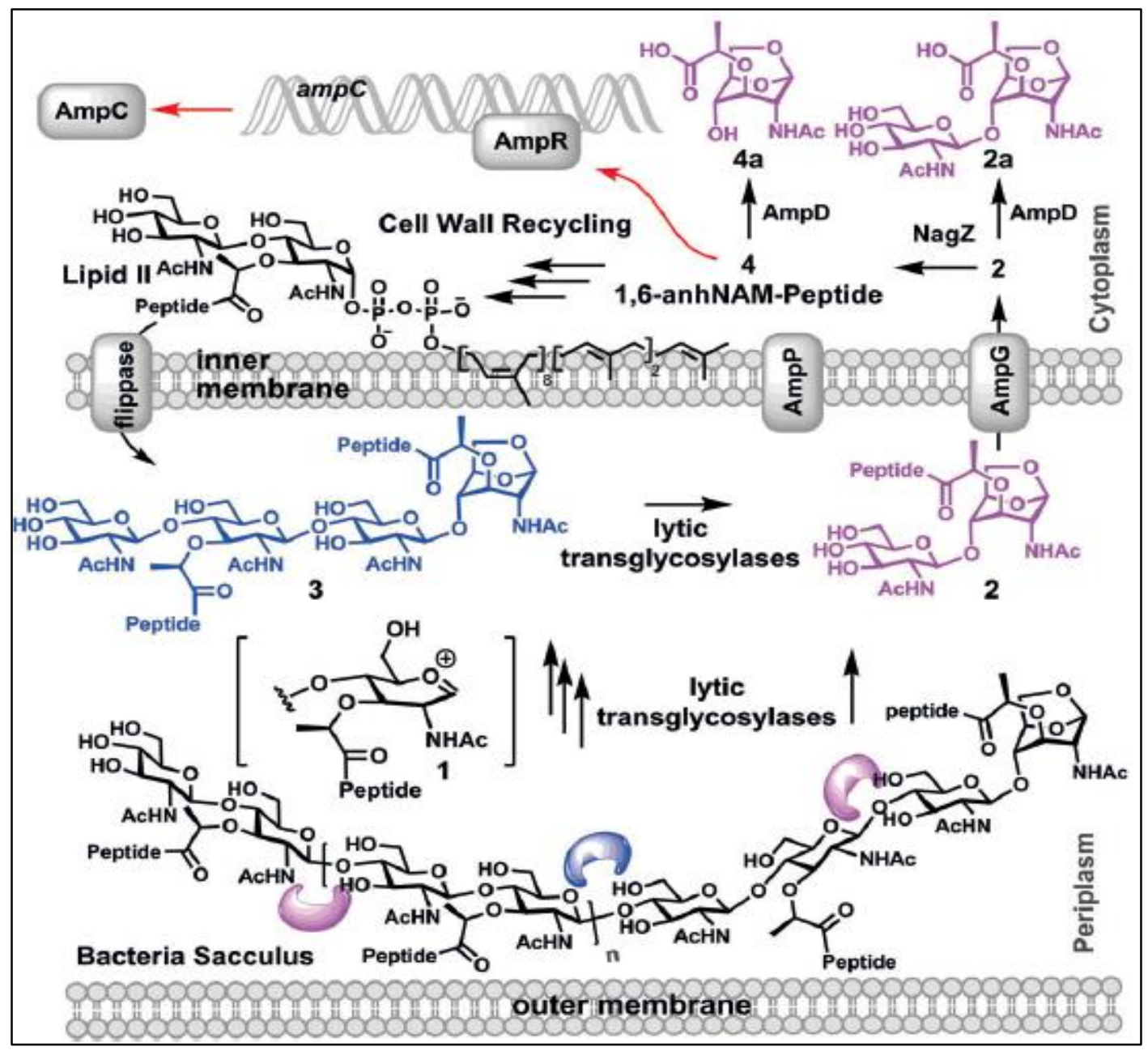

Figure 2.1. Muropeptides in recycling. The reactions of LTs produce muropeptides containing the 1, 6-anhydromuramyl moiety, which are translocated by the permease AmpG (and AmpP in P. aeruginosa). In normal growth of the bacteria, 4 would initiate recycling events to regenerate lipid II. However, as an offshoot of the recycling events, accumulation of 4 induces $\beta$ lactamase (AmpC) expression (indicated by red arrows). 
al., 2015). The functions in resistance to $\beta$-lactam antibiotics involve binding to the gene regulator AmpR, which allows for transcription of the gene ampC for the Gram-negative AmpC $\beta$-lactamase (Figure 2. 1) (Jacobs, et al., 1997, Kong, et al., 2005). $\beta$-Lactamase hydrolyzes $\beta$-lactams antibiotics, which inhibit the action of penicillin-binding proteins by mimicking the structure of the terminal d-Ala-d-Ala in the stem peptides of the peptidoglycan.

The processes that muropeptides mediate are not fully understood due to impediments such as the minute quantities, rapid metabolic flux, and complexity of the structures. Once the structures are elucidated, they need to be prepared in the laboratory for validation of the assigned structure and for the conduct of biochemical studies. Here, we report the identification, characterization, and quantification of several muropeptides from periplasm of $P$. aeruginosa, an opportunistic human pathogen.

\subsection{Materials and methods}

2.4.1 Strains, media and growth conditions. Pseudomonas aeruginosa PAO1 and $P$. aeruginosa Z61 (ATCC 35151) were used in this study. P. aeruginosa was cultured in Luria Bertani broth (5 g tryptone, $10 \mathrm{~g}$ sodium chloride, and $5 \mathrm{~g}$ yeast extract per liter) supplemented with $1.5 \%$ agar when needed at $37^{\circ} \mathrm{C}$ (Holloway, 1955). The antibiotic cefoxitin was added when required.

2.4.2 Induction and $\beta$-lactamase assay. Cefoxitin at half minimal-inhibitory concentration $(512 \mu \mathrm{g} / \mathrm{mL})$ was used for induction of $\beta$-lactamase in $P$. aeruginosa PAO1 (Livermore, 2002). The antibiotic was added when cell culture was at $\mathrm{OD}_{600} 0.2$ and grown until $\mathrm{OD}_{600}$ of 1.2. The culture was harvested, washed once and resuspended in $50 \mathrm{mM}$ sodium phosphate buffer $(\mathrm{pH}$ 8). The suspension was sonicated. The sonicated 
suspension was centrifuged, and the supernatant was used for analysis of $\beta$-lactamase activity. A $7.5-\mu \mathrm{L}$ portion of the supernatant of both induced and non-induced samples were added to $1 \mathrm{~mL}$ of the assay buffer with nitrocefin in a final concentration of $100 \mu \mathrm{M}$. The absorbance at $500 \mathrm{~nm}$ was observed for $5 \mathrm{~min}$ (O'Callaghan, et al., 1972). The $\triangle \mathrm{A} 500 / \mathrm{min}$ was recorded for both induced and non-induced solution. The protein concentrations of the supernatants were estimated by BCA assay (Walker, 1994). The enzyme activity was expressed as the nanomole of nitrocefin hydrolyzed per min per mg of protein (Kong, et al., 2005). Compounds $2 \mathrm{e}$ and $4 \mathrm{e}$ were used for induction of $\beta$ lactamase in $P$. aeruginosa Z61 (ATCC 35151) at $100 \mu \mathrm{g} / \mathrm{mL}$. Cefoxitin at quarter minimal-inhibitory concentration $(0.0156 \mu \mathrm{g} / \mathrm{mL})$ was used as control. Compounds $2 \mathrm{a}$ and $4 \mathrm{a}$ were used (as negative control). The culture was grown to $\mathrm{OD}_{600}$ of $0.1\left(\sim 10^{8}\right.$ $\mathrm{CFU} / \mathrm{mL}$ ) in LB medium and, subsequently, diluted 100-fold. LB medium $(2.5 \mathrm{~mL})$ containing the compound or the antibiotics were inoculated with $2.5 \mathrm{~mL}$ of the inoculum. Various cultures were allowed to grow within the range of $\mathrm{OD}_{600}$ of $\sim 1.0-1.8$. The rest of the protocol is the same as for the wild type. The result is summarized in Table 2

2.4.3. Bacterial cell counting. $P$. aeruginosa $\mathrm{PAO} 1$ was grown in LB broth until early stationary phase $\left(\mathrm{OD}_{600}\right.$ of 1.2) was reached. Serial dilutions of the bacterial culture were plated on LB agar plates and incubated at $37^{\circ} \mathrm{C}$ for $16-18 \mathrm{~h}$. Colonies were counted and expressed as colony-forming units (CFU) per mL. Only plates with 30-300 colonies were considered. The average number was $4.9 \times 0.5 \times 10^{8} \mathrm{cell} / \mathrm{mL}$ of culture.

2.4.4 Syntheses of authentic muropeptides. The chemical structure of authentic compounds used in this study is given in Chart S1. Synthetic methods for $\mathbf{2 e}, \mathbf{4 c}, \mathbf{4 d}, \mathbf{4 e}$, 7, and 8 were reported previously by Dr. Mobashery's laboratory and were followed for the samples used in the present study (Hesek, et al., 2009, Lee, et al., 2009, Lee, et al., 
2010). Compound 4 a was synthesized for this study by catalytic hydrogenation of the intermediate 9. 1H NMR (500 MHz, CD3CN) ס 1.37 (d, J =6.8 Hz, 3H), $1.93(\mathrm{~s}, 3 \mathrm{H}), 3.37$ (s, 1H), 3.55 - $3.72(\mathrm{~m}, 3 \mathrm{H}), 3.90(\mathrm{~d}, \mathrm{~J}=8.4 \mathrm{~Hz}, 1 \mathrm{H}), 4.11(\mathrm{~d}, \mathrm{~J}=7.4 \mathrm{~Hz}, 1 \mathrm{H}), 4.24(\mathrm{q}, \mathrm{J}=$ $6.8 \mathrm{~Hz}, 2 \mathrm{H}), 4.50(\mathrm{~d}, \mathrm{~J}=5.2 \mathrm{~Hz}, 1 \mathrm{H}), 5.31(\mathrm{~s}, 1 \mathrm{H}), 6.79(\mathrm{~d}, \mathrm{~J}=8.6 \mathrm{~Hz}, 1 \mathrm{H}) ; 13 \mathrm{C} N M R$ (126 MHz, CD3CN) d 18.7, 23.0, 50.5, 66.1, 69.7, 74.8,77.2, 79.8, 101.5, 118.3, 171.3, 175.0; HRMS (ESI-QTOF) $\mathrm{m} / \mathrm{z}[\mathrm{M}+\mathrm{H}]+$ Calcd 276.1078 for $\mathrm{C} 11 \mathrm{H} 17 \mathrm{NO}$; found 276.1101.Compound $\mathbf{2 a}$ was obtained from incubation of compound $\mathbf{2 e}$ with an amidase AmpDh3 of $P$. aeruginosa (Martinez-Caballero, et al., 2013, Zhang, et al., 2013). The reaction product $2 \mathrm{a}$ was confirmed by high-resolution mass spectrometry, which is consistent with the chemical formula of C19H30N2O12: HRMS (ESI-QTOF) m/z [M+H]+ Calcd 479.1872; found 479.1844. Its chemical nature was further confirmed by LC/MS/MS experiment (Figure S1A). Compound $\mathbf{2} \mathbf{d}$ was obtained from incubation of compound $2 \mathrm{e}$ with penicillin-binding protein 4 of $P$. aeruginosa. [9] The reaction product 2d was confirmed by high-resolution mass spectrometry, which is consistent with the chemical formula of C37H59N7O2: HRMS (ESIQTOF) m/z [M+H]+ Calcd 922.3888; found 922.3840. Its chemical nature was further confirmed by LC/MS/MS experiment (Figure S1B). 


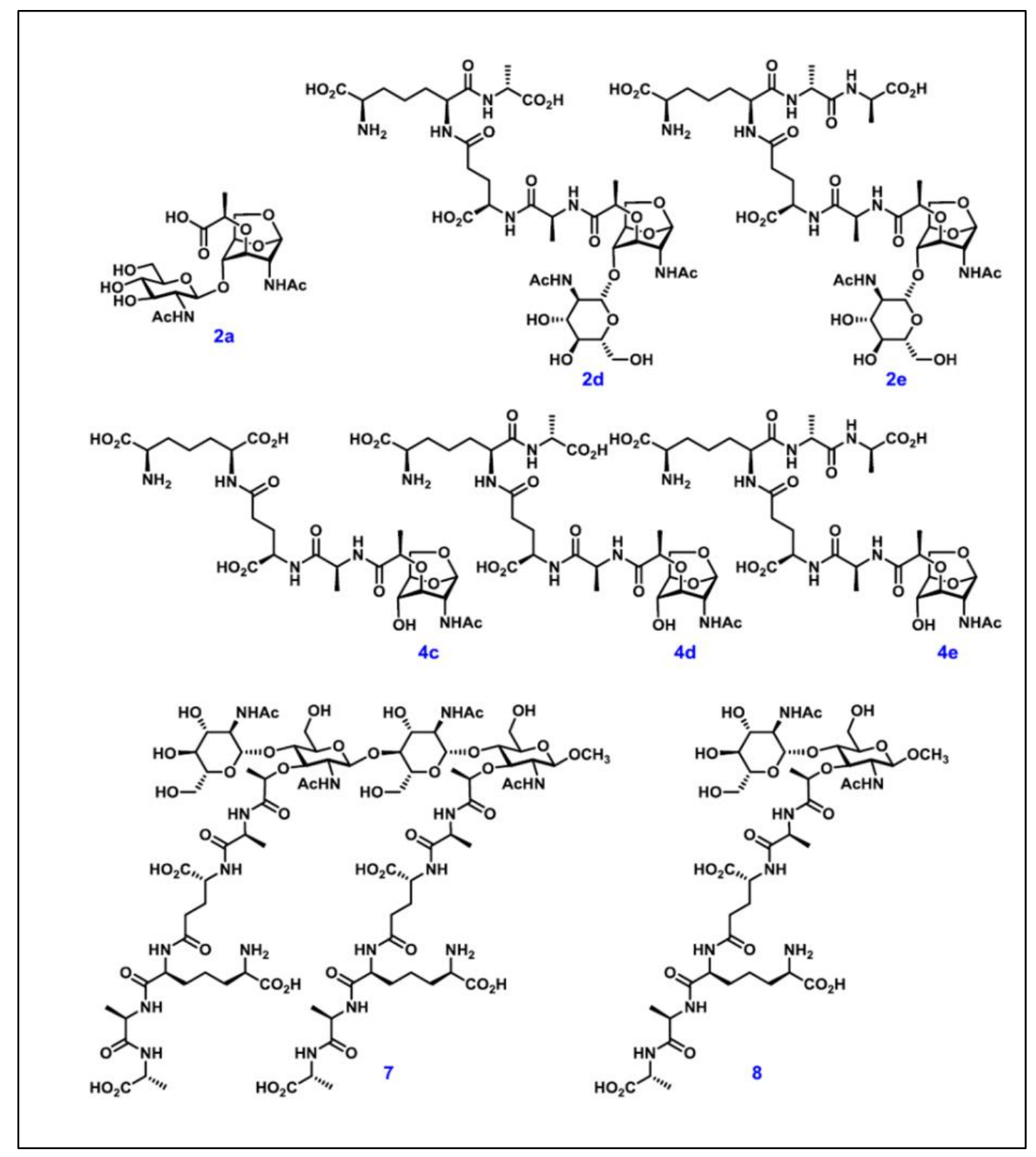

Figure 2.2. The chemical structure of authentic muropeptides used in this study. 


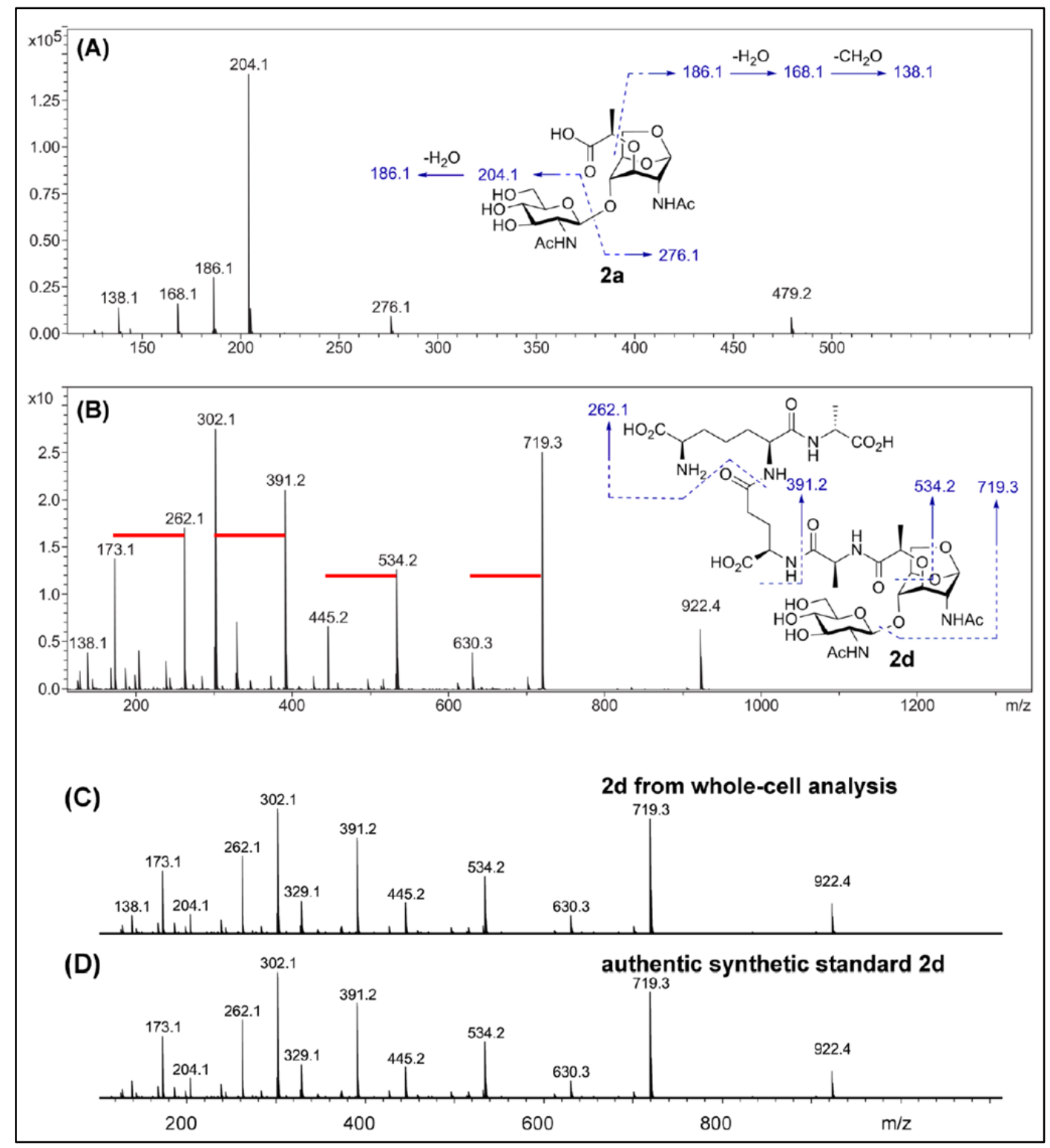

Figure 2.3. Collision-induced dissociation spectra. Of $\mathbf{2 a}(\mathbf{A}), \mathbf{2 d}(\mathbf{B})$, and side-by side comparison to authentic synthetic standard $2 d(C$ and $\mathbf{D})$. Losses of Ala are indicated in red bar in (B). 


\subsubsection{Sample preparation: release of muramyl peptides from whole-cell. $P$.}

aeruginosa PAO1 with and without induction (cefoxitin $512 \mu \mathrm{g} / \mathrm{mL}$ ) was grown until late log to early stationary phase $\left(\mathrm{OD}_{600} 1.2\right)$. The culture was harvested by centrifugation at $4,500 \mathrm{~g}$ for $20 \mathrm{~min}$ and the supernatant discarded. The cell pellet was resuspended gently in cold sucrose solution (20\% sucrose, $1.2 \mathrm{mM}$ EDTA, $30 \mathrm{mM}$ Tris, $\mathrm{pH} 8$ ) and left at $4{ }^{\circ} \mathrm{C}$ for $10 \mathrm{~min}$. This was centrifuged, and the pellet resuspended in ice-cold water. The suspension was incubated at $4^{\circ} \mathrm{C}$ for $10 \mathrm{~min}$, and then centrifuged at $11,000 \mathrm{~g}$ for $20 \mathrm{~min}$ and the supernatant was discarded. The pellet was then boiled for $3 \mathrm{~min}$ and sonicated to ensure complete release of muropeptides. The sample was filtered using the $30-\mathrm{kDa}$ molecular weight cut off mini-column. The flow-through was boiled for 6 min to remove any residual enzymatic activity, concentrated by speed vacuum at $50{ }^{\circ} \mathrm{C}$ and used for LC/MS analyses.

2.4.6 Detection and quantification of muropeptides by LC/MS. LC/MS conditions for muropeptide detection corresponded to those in the method developed by Dr. Mobashery's laboratory (Lee, et al., 2013). Peak areas from extracted ion chromatograms of corresponding muropeptides were integrated and normalized to internal standard (compound 8). Quantification of muropeptide was done from the standard curve of synthetic compound $\mathbf{2 e}$. Standard curves for $\mathbf{2 e}, \mathbf{4 c}, \mathbf{4 d}, \mathbf{4 e}$, and $\mathbf{7}$ were determined and they were very similar within $7 \%$ variation of each other (Figure S2). The collection of our synthetic standards covers distinctive chemical structures of $>95 \%$ of detected muropeptide. So, we chose $2 \boldsymbol{e}$ as a synthetic standard for quantification, as it has the closest structure to $\mathbf{2} \mathbf{d}$, the most abundant detected species. 


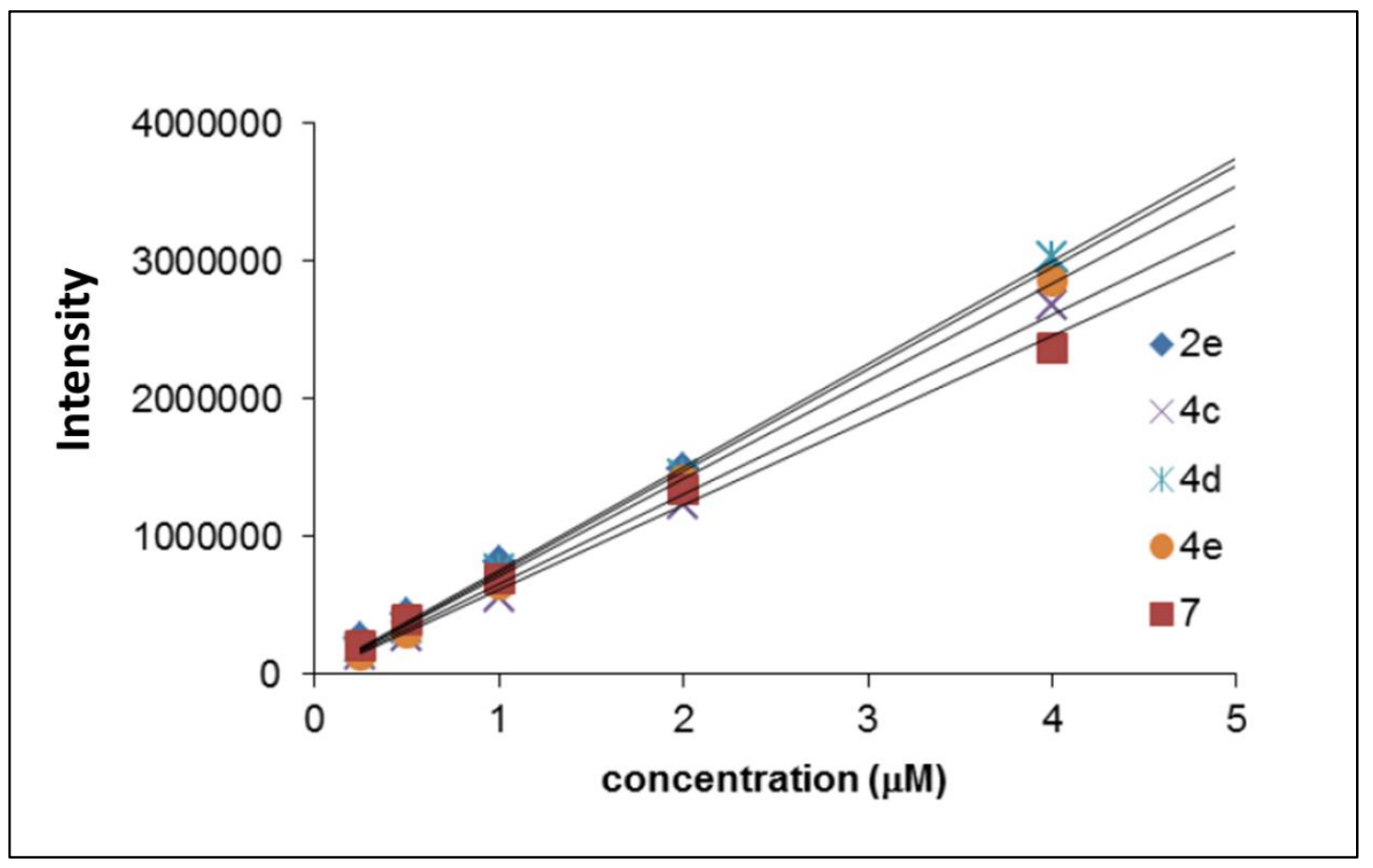

Figure 2.4. Standard curves of synthetic standards, $2 e, 4 c, 4 d, 4 e$, and 7.

2.4.7 Calculation of number of muropeptides per cell. The concentration of $\mathbf{2 d}$ was calculated from the standard curve of authentic standard $2 \mathbf{e}$, which is $4.5 \times 10^{-11} \mathrm{~mol} / \mathrm{mL}$ of culture. This was converted to $\mathrm{mol} / \mathrm{cell}(\mathrm{x})$ using the value obtained from bacterial cell counting, described above. The value $x$ then was converted to number of molecules $(y)$ using Avogadro's number, $6.022 \times 10^{23} / \mathrm{mol}$. The rest of muropeptides were calculated the same way as $\mathbf{2} \mathbf{d}$ and numbers are given in Table 1.

$x=4.5 \times 10^{-11} \mathrm{~mol} / \mathrm{mL}$

$4.9 \times 10^{8} \mathrm{cell} / \mathrm{mL}=9.2 \times 10^{-20} \mathrm{~mol} / \mathrm{cell}$

$1: 6.022 \times 10^{23}=9.2 \times 10^{-20} \mathrm{~mol}: \mathrm{y}$

$y=5.5 \times 10^{4}$ molecules $/$ cell 


\subsection{Results and Discussion}

Sample preparation is important, as dilution of the minute quantities and contamination could confound analysis. Initially, osmotic shock was used for liberation of the periplasmic content that contains the muropeptides. This would have separated the periplasmic and cytoplasmic metabolites before attempt at isolation of muropeptides. Unfortunately, $10-30 \%$ cytoplasmic contamination was noted in these samples. The results were also not reproducible, and muropeptides could merely be identified near the detection limit of 0.4 pmol by our instrumentation.

The muropeptides that enter the cytoplasm via the permease AmpG (or AmpP) are expected to undergo rapid metabolic flux (Figure 2.1). This assertion was documented by preparing spheroplasts of $P$. aeruginosa PAO1. The cytoplasmic content from the lysed spheroplasts were analyzed by LC/MS for muropeptides. None could be detected, suggesting that the cytoplasmic muropeptides were rapidly metabolized to Lipid II, with concentrations below our detection limit. Hence, the whole bacterium was

grown and lysed, an approach that proved to be reliable and reproducible. The muropeptides that were generated under these conditions could only have come from the intact periplasm in the whole bacterium. After sample preparation, the LC/MS analyses were performed for detection and identification of muropeptides. The muropeptide content of the whole cells of $P$. aeruginosa PAO1 was compared in the absence and presence of half of the minimal-inhibitory concentration (MIC) of antibiotic cefoxitin, a $\beta$-lactam that interferes with cell-wall synthesis (Livermore, 1995). Cefoxitin at sub-MIC levels activates the expression of $\beta$-lactamase efficiently, thereby leading to antibiotic resistance in $P$. aeruginosa. This is believed to be mediated by a messenger 
function of a muropeptide. Hence, one or more of the muropeptides listed in Figure $2 \mathrm{~A}$ is expected to serve as the signaling molecule for antibiotic resistance.

As shown in Figure 2A, compounds are numbered according to the nature of the sugar, 2 for NAG-1,6-anhNAMand 4 for 1,6-anhNAM. For the peptide component, a has no peptide, b, c, d, and e carry di-, tri-, tetra- and pentapeptide, respectively (full pentapeptide is I-Ala-y-d-Glu-m-DAP-d-Ala-d-Ala; bottom right of Figure 2A). Compounds 3 are (NAG-NAM-peptide) n-NAG-1,6-anhNAM-peptide and compounds 5 are for cross-linked species. For example, compound $\mathbf{5 d d}$ indicates cross-linked peptide between two tetrapeptides. Compounds with reducing-end sugars, which lack the 1,6anhNAM, were also detected as minor components and are designated with the letter $\mathrm{R}$.

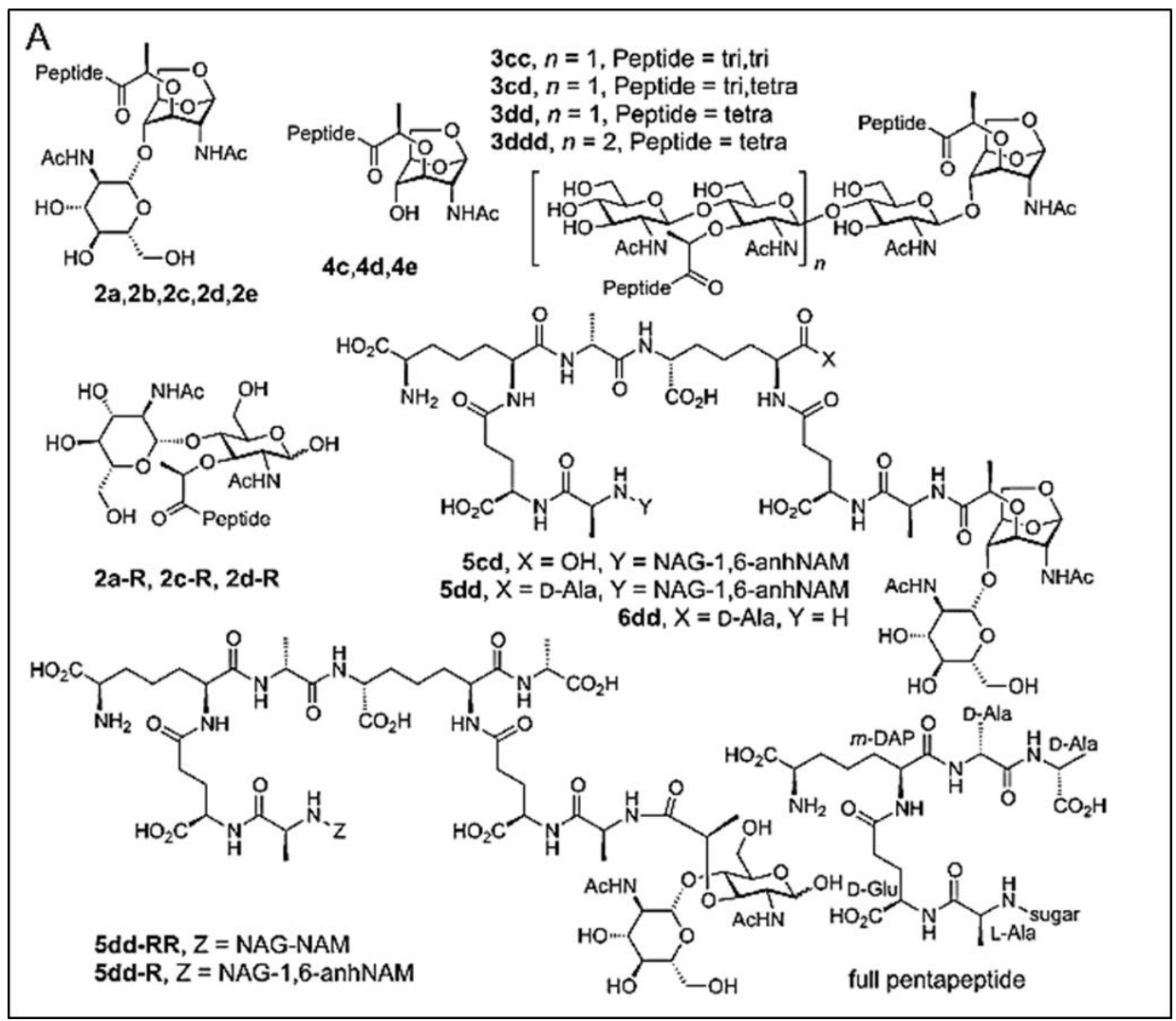




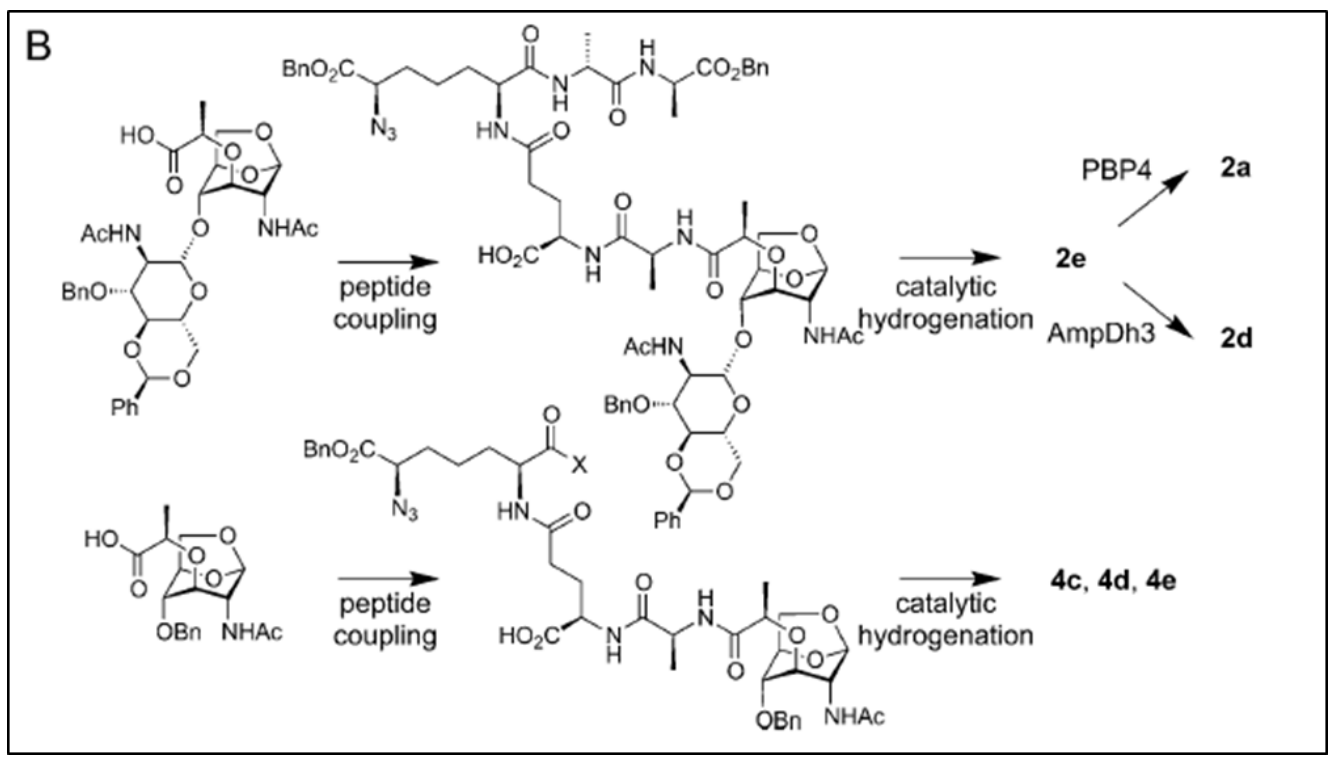

Figure 2. 5. A) Chemical structures of detected muropeptides. B) The chemoenzymatic syntheses of six muropeptides.

Four muropeptides, 2e, (Hesek, et al., 2009) 4c, 4d, and 4e (Lee, et al., 2009, Zhang, et al., 2013) were synthesized. A few of these authentic samples were also converted to new species by known enzymatic reactions (Figures. 2B and S1). For example, $\mathbf{2 e}$ was converted to $\mathbf{2} \mathbf{d}$ by the use of penicillin-binding protein 4 (PBP4),(Lee, et al., 2015) and $\mathbf{2 e}$ to $\mathbf{2 a}$ using AmpDh3,(Zhang, et al., 2013) both purified recombinant enzymes from $P$. aeruginosa. Figures. 3C-3F show EICs of the detected metabolites $2 \mathbf{a}$, 2d, 2e, 4c, and $\mathbf{4 d}$, and their comparison to the authentic standards (Figure 3G). Analysis was further done with comparison of MS and MS/MS with authentic samples, as exemplified in Figure S1. For structure assignment of metabolites whose authentic standards were not available, the method that was developed previously by Dr. Mobashery's laboratory to analyze turnover products of sacculus by LTs and PBP4 was used (Lee, et al., 2013, Lee, et al., 2015).

Quantification was done by integrating peak areas from EICs of the corresponding $\mathrm{m} / \mathrm{z}$ values of the individual muropeptide. This was converted to 
concentration using standard curves generated with the authentic $\mathbf{2 e}$. The concentration was converted to numbers of molecules (of each compound) per bacterium (Table 1; SI). Standard curves for $\mathbf{2 e}, \mathbf{4 c}, \mathbf{4 d}, \mathbf{4 e}$ and $\mathbf{7}$ ( $\beta$-methoxy-NAG-NAM (pentapeptide)-NAGNAM (pentapeptide))(Lee, et al., 2010) were very similar within 7\% variation of each other (Chart S1 and Figure S2). The collection of our synthetic standards covers distinctive chemical structures of $>95 \%$ of the detected muropeptides. So, $2 \mathbf{e}$ was chosen as a representative synthetic standard for quantification. The most abundant muropeptide in wild-type PAO1 strain is NAG-1, 6-anhNAM-tetrapeptide (2d). The di-, tri-, and pentapeptide variants $(\mathbf{2 b}, \mathbf{2 c}$, and $\mathbf{2 e}$ ) are also found, along with $\mathbf{2 a}$ (with no peptide). These are reaction products of LTs, mostly from the exolytic activity. The discovery of compounds with the core 1,6-anhNAM-peptides (4c and $\mathbf{4 d}$ ) suggests the existence of the $\mathrm{N}$-acetylglucosaminidase activity in $P$. aeruginosa. The presence of such an enzyme (FlgJ) in the periplasm was recently documented in Salmonella enterica (Herlihey, et al., 2014). This activity in P. aeruginosa might be mediated by PA1085, which has an identity of $31 \%$ and a similarity of $46 \%$ at the amino-acid level to FlgJ from S. enterica (Figure S3) (Herlihey, et al., 2014).To our knowledge this is the first documentation of a periplasmic $\mathrm{N}$-acetylglucosaminidase reaction product in $P$. aeruginosa (Herlihey, et al., 2014). Oligomeric sugars (up to hexamers) with tetrapeptide (3dd and 3ddd) or a mix of tetra and tripeptide (3cd and 3cc) were also found. These are products of the endolytic reactions of LTs (Johnson, et al., 2013). 


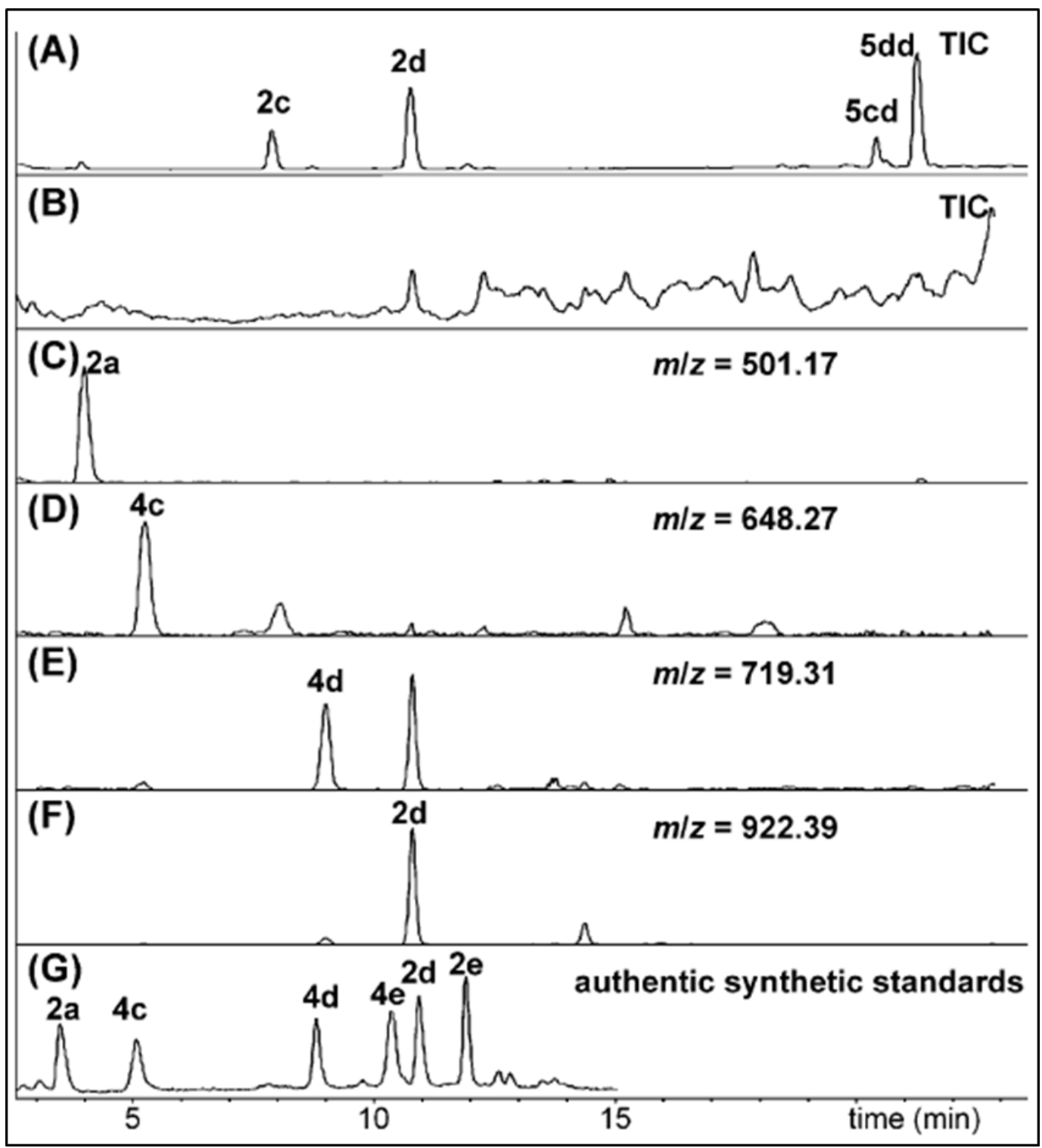

Figure 2.6. Analysis of $\boldsymbol{P}$. aeruginosa muropeptides. The LC-MS TICs of sacculus digested by MltA (A) and whole-cell analysis (B). EICs of 2a (C), 4c (D), 4d (E), and 2d (F) from whole-cell samples, and the TIC of authentic synthetic standards mixed together (G). 


\begin{tabular}{|c|c|c|c|}
\hline Muropeptide & Wild-type & Induced ${ }^{[b]}$ & $p$-value $\mathrm{e}^{[c]}$ \\
\hline \multicolumn{4}{|c|}{ NAG-1,6-anhNAM } \\
\hline $2 \mathrm{a}$ & $1.5 \pm 0.07$ & $0.3 \pm 0.01$ & $0.01 *$ \\
\hline $2 \mathrm{~b}$ & $0.2 \pm 0.01$ & $0.08 \pm 0.01$ & $0.004 *$ \\
\hline $2 c$ & $0.8 \pm 0.05$ & $0.3 \pm 0.02$ & $0.02 *$ \\
\hline $2 \mathrm{~d}$ & $5.5 \pm 0.3$ & $1.0 \pm 0.3$ & $0.002 *$ \\
\hline $2 \mathrm{e}$ & $0.01 \pm 0.005$ & $0.1 \pm 0.02$ & $0.04 *$ \\
\hline \multicolumn{4}{|l|}{ 1,6-anhNAM } \\
\hline $4 c$ & $0.3 \pm 0.06$ & $0.1 \pm 0.003$ & $0.0003 *$ \\
\hline $4 d$ & $0.4 \pm 0.01$ & $0.07 \pm 0.003$ & $0.01 *$ \\
\hline $4 \mathrm{e}$ & $N . D .^{[d]}$ & $0.08 \pm 0.01$ & $0.04 *$ \\
\hline \multicolumn{4}{|c|}{$(\text { NAG-NAM })_{n}$-NAG-1,6-anhNAM } \\
\hline $3 c c$ & $0.1 \pm 0.01$ & $0.02 \pm 0.006$ & $0.003 *$ \\
\hline $3 \mathrm{~cd}$ & $0.2 \pm 0.02$ & $0.03 \pm 0.007$ & $0.03 *$ \\
\hline $3 \mathrm{dd}$ & $0.9 \pm 0.06$ & $0.09 \pm 0.03$ & $0.002 *$ \\
\hline 3 ddd & $0.1 \pm 0.02$ & $0.01 \pm 0.003$ & $0.049 *$ \\
\hline \multicolumn{4}{|c|}{ NAG-1,6-anhNAM-crosslinked-1,6-anhNAM-NAG } \\
\hline $5 \mathrm{~cd}$ & $0.03 \pm 0.004$ & $0.02 \pm 0.001$ & 0.2 \\
\hline $5 \mathrm{dd}$ & $0.3 \pm 0.01$ & $0.07 \pm 0.007$ & $0.001 *$ \\
\hline $6 \mathrm{dd}$ & $0.04 \pm 0.001$ & $0.02 \pm 0.004$ & 0.07 \\
\hline \multicolumn{4}{|l|}{ Reduced end } \\
\hline $2 a-R$ & $0.07 \pm 0.01$ & N.D..$^{[d]}$ & $0.02 *$ \\
\hline $2 c-R$ & $0.07 \pm 0.03$ & $0.02 \pm 0.002$ & 0.11 \\
\hline $2 d-R$ & $0.5 \pm 0.1$ & $0.1 \pm 0.03$ & 0.06 \\
\hline $5 \mathrm{dd}-\mathrm{RR}$ & $0.1 \pm 0.002$ & $0.01 \pm 0.002$ & $0.0002 *$ \\
\hline $5 \mathrm{dd}-\mathrm{R}$ & $0.02 \pm 0.002$ & $0.009 \pm 0.002$ & $0.047 *$ \\
\hline Total $\left.\right|^{[\mathrm{e}]}$ & $11.1 \pm 0.8$ & $2.4 \pm 0.5$ & \\
\hline
\end{tabular}

Table 2.1 Detected muropeptides from whole-cell analysis (in molecules per bacterium $\times \mathbf{1 0}^{4}$ [a] Average of two runs. [b] PAO1 was exposed to cefoxitin at 512 $\mathrm{mg} / \mathrm{mL}^{1}$. [c] $\mathrm{p}$-values from Students t-test. * Significant difference $(\mathrm{p}<0.05)$ between the wild-type and induced samples. [d] Not detected. [e] Total detected muropeptides. 
Cross-linked muropeptides such as $5 \mathbf{c d}, 5 \mathbf{d d}$, and $6 \mathbf{d d}$ were also found. As minor components, muropeptides containing a sugar with a reducing end (2a-R, $\mathbf{2 c}-\mathbf{R}$, 2d-R, 5dd-RR, and $\mathbf{5 d d - R}$ ) were also detected. This indicates that the reactive oxocarbenium species partitions between either entrapment of the internal C6-hydroxyl or of a water molecule, or there exists a yet-to-be identified hydrolytic glycosidase in this organism. The ratio of the two types of products (non-reducing to reducing) is $\sim 14: 1$.

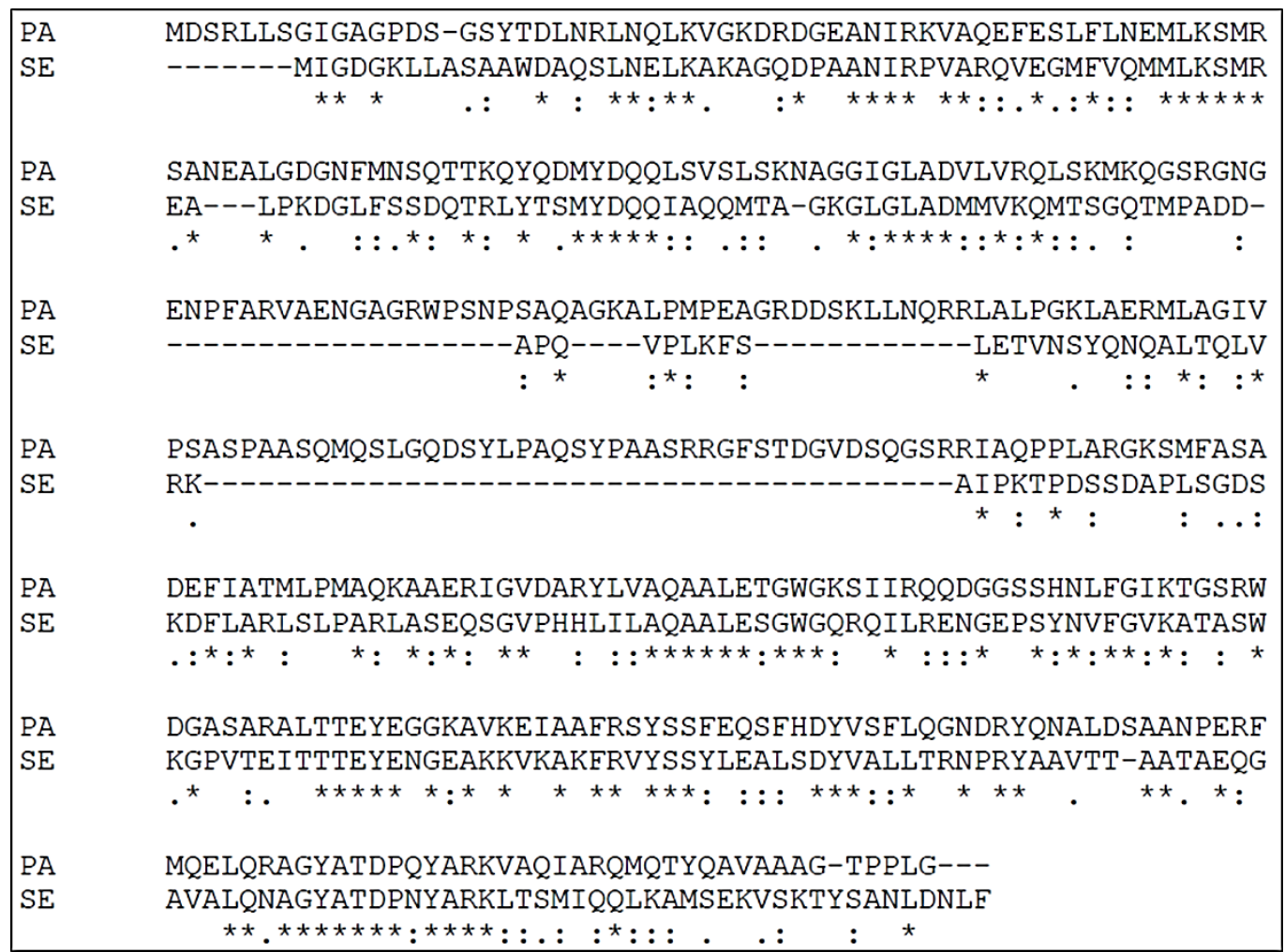

Figure 2.7. Alignment of PA1085 (PA) and FlgJ (SE) sequence

Same sample preparation and analyses were carried out with $P$. aeruginosa PAO1 exposed to a cell-wall-active antibiotic cefoxitin at half of the MIC (i.e., 512 $\mu \mathrm{g} / \mathrm{mL}$ ), hence a non-lethal concentration (Livermore, 1995). The exposure to the antibiotic alters the pool of muropeptides, where one or more is understood to enter the cytoplasm via AmpG (or AmpP) permease and upregulate production of $\beta$-lactamase, 
the antibiotic-resistance determinant (Kong, et al., 2010, J. F. Fisher, 2015). The induction of resistance was confirmed by the $\beta$-lactamase assay using nitrocefin.

The same number of bacteria and the same conditions were used in both cases; hence, the values of the two columns of Table 1 can be compared to each other. The analysis showed that the total muropeptide (molecules/bacterium) was significantly reduced ( $p$-value $<0.05$ by Student's t-test) in the induced vs the uninduced case: 24,000 vs. 111,000 (Table 1). The most abundant muropeptide in the uninduced sample was 2d (NAG-1, 6-anhNAM-tetrapeptide). Muropeptide 2e (NAG-1, 6-anhNAMpentapeptide) was enriched at 46-fold upon antibiotic induction (1000 in 24,000 molecules vs. 100 in 111,000 molecules). That is to say that the $\beta$-lactam antibiotic inhibits the targeted PBP, whose lack of activity leaves its peptidoglycan substrate in the sacculus intact. This observation in living bacteria agrees with the finding of the in vitro sacculus analysis of the induced $P$. aeruginosa (Moya, et al., 2012, Lee, et al., 2015). Compound $4 \mathrm{e}$ was detected only in the induced sample. This observation suggests that as the concentration of $\mathbf{2 e}$ increased upon induction, the compound was likely turned over by the aforementioned $\mathrm{N}$-acetylglucosaminidase to produce $4 \mathbf{e}$. Other than $\mathbf{2 e}$ and $\mathbf{4 e}$, the rest of muropeptides detected in the induced sample were similar to those in the uninduced. It is not immediately obvious as to why the total quantity of muropeptides is lower in the induced sample (one fifth of the uninduced). This likely reflects the altered cell-wall modifications of the bacterium in the presence of the sub-lethal concentration of the antibiotic.

The obvious question now becomes whether exogenously added authentic muropeptides $2 \mathbf{e}$ or $\mathbf{4 e}$ could cause induction of antibiotic resistance in the absence of antibiotic. We investigated this first with the wild-type $P$. aeruginosa PAO1 strain. 
Addition of muropeptide $2 \mathrm{e}$ or $4 \mathrm{e}$ at upwards of $500 \mu \mathrm{g} / \mathrm{mL}$ failed to induce $\beta$-lactamase expression (Table 2.2). Going with the premise that the Gram-negative outer membrane is a formidable barrier to penetration of most small molecules into the periplasm, we procured a mutant strain defective in its outer membrane. The strain $P$. aeruginosa Z61 has the full complement of the genes necessary for induction of $\beta$-lactamases, but it expresses a mutant version of the $\beta$-lactamase with diminished activity (Angus, et al., 1982). Nonetheless, using the nitrocefin assay we observed a 4.7 -fold increase of induction of $\beta$-lactamase at quarter-MIC level of cefoxitin.

\begin{tabular}{|c|c|c|c|c|c|c|}
\hline \multirow{3}{*}{$\begin{array}{l}\text { Inducer } \\
\text { Cefoxitin }^{[d]}\end{array}$} & \multicolumn{3}{|c|}{ Wild-type (PAOI) } & \multicolumn{3}{|c|}{ Mutant $(Z 61)^{[b]}$} \\
\hline & \multirow{2}{*}{$\begin{array}{l}\text { Conc. } \\
{\left[\mu \mathrm{gLL}^{-1}\right]} \\
512\end{array}$} & \multicolumn{2}{|c|}{$\begin{array}{l}\beta \text {-lactamase } \\
\text { induction }^{[c]}\end{array}$} & \multirow{2}{*}{$\begin{array}{l}\begin{array}{l}\text { Conc. } \\
{\left[\mu \mathrm{mL}^{-1}\right]}\end{array} \\
0.0156\end{array}$} & \multicolumn{2}{|c|}{$\begin{array}{l}\beta \text {-lactamase } \\
\text { induction }^{[c]}\end{array}$} \\
\hline & & $136^{[e]}$ & $1555 / 11.4$ & & $4.7^{[e]}$ & $4.6 / 0.98$ \\
\hline $2 \mathrm{e}$ & 500 & 1.0 & 19.1/19.1 & 100 & $1.4^{[e]}$ & $27.5 / 20.0$ \\
\hline $4 \mathrm{e}$ & 500 & 1.0 & 19.1/19.1 & 100 & $1.7^{[\mathrm{el}}$ & $5.5 / 3.3$ \\
\hline $2 a^{[d]}$ & & $L^{[f]}$ & & $10^{[\mathrm{gl}]}$ & 1.0 & $4.1 / 4.0$ \\
\hline $4 \mathrm{a}^{[\mathrm{d}]}$ & & $-^{[\mathrm{f}]}$ & & 100 & 1.0 & $3.6 / 3.5$ \\
\hline
\end{tabular}

Table 2.2. Induction of $\boldsymbol{\beta}$-lactamase activity by three inducers [a] Average of two runs. [b] The strain is defective in its outer membrane. [c] The number (the left column) is calculated from $\beta$-lactamase activity in the presence of an inducer divided by that without an inducer (the right column) under the same conditions. The enzyme activity was expressed as nmol of nitrocefin hydrolyzed per min per $\mathrm{mg}$ of protein for wild-type and $\mathrm{pmol} / \mathrm{min} / \mathrm{mg}$ for mutant. [d] Cefoxitin and compounds $\mathbf{2 a}$ and $\mathbf{4 a}$ were used as positive and negative controls for induction, respectively. [e] $\beta$-Lactamase activities were significantly different $(p<0.05)$ between induced and uninduced samples. [f] Not measured. [g] Owing to limited supply of compound, we could only assess the effect at the lower concentration.

The same experiment performed with the bacteria exposed to $100 \mu \mathrm{g} / \mathrm{mL}$ muropeptides $\mathbf{2 e}$ or $\mathbf{4 e}$ resulted in 1.4 or 1.7 induction, respectively (Table $\mathrm{S} 1$ ). This is a large excess of these compounds, but we used them so, as we had expected that the 
exogenously added compounds would undergo turnover by periplasmic enzymes. Hence, the two metabolites-both produced in response to the exposure of bacteria to the antibiotic inducer cefoxitin-collectively account for most of the induction observed by cefoxitin. We note that compound $\mathbf{2 a}$ and $\mathbf{4 a}$ (metabolites without a peptide stem; products of the reaction of AmpD) as negative controls. As expected, under the same conditions, induction was not observed. Activity of AmpD (Figure 1) is at the crossroads of induction of resistance vs. cell-wall recycling (reversal of induction).

This study reports the nature and quantities of 20 muropeptides from $P$. aeruginosa. The levels of muropeptide $2 \mathrm{e}$ became elevated by 46 -fold on exposure of bacteria to sub-MIC levels of a good inducer, cefoxitin. We also observed muropeptide $4 e$ only in the induced cells. This study discloses that authentic synthetic samples of muropeptides $\mathbf{2 e}$ and $\mathbf{4 e}$ could serve as inducers of $\beta$-lactam-antibiotic resistance in the absence of antibiotic. These experiments clearly document that at least muropeptides $\mathbf{2 e}$ and $\mathbf{4 e}$ are chemical elicitors of induction of antibiotic resistance.

\subsection{Acknowledgements}

This work was supported by grant GM61629 from the NIH (to SM) and in-part by NSF IIP-1237818 [PFI-AIR: CRESTI/UCRC-Industry Ecosystem to Pipeline Research] (KM), Florida International University (FIU) Bridge Funding (KM), FIU Herbert Wertheim College of Medicine Graduate Assistantship (SD). 
Chapter 3

Role of $P$. aeruginosa permeases PA4393 and PA4218 in antibiotic resistance and cell-wall recycling 


\subsection{Abstract}

Pseudomonas aeruginosa is well-known for its intrinsic as well as acquired resistance to multitude of antibiotics including fluoroquinolones, aminoglycosides, macrolides and the $\beta$-lactams. A frequent mechanism of resistance to $\beta$-lactams is through expression of the hydrolytic enzymes, $\beta$-lactamases. Expression of the major $\beta$ lactamase AmpC is regulated by a LysR-type transcriptional regulator AmpR, contingent upon its binding with specific muropeptides. In $P$. aeruginosa, there are two inner membrane permeases that may serve as muropeptide transporters; PA4393 (AmpG) and PA4218 (AmpP). It was postulated that AmpG imports the muramyl peptides into the cytoplasm whereas AmpP exports into the periplasm. In this research, we investigate the role of AmpG and AmpP in antibiotic resistance and as muropeptide transporters. To address their role three in-frame deletion mutants were constructed $\triangle a m p G, \triangle a m p P$, and $\triangle a m p G \triangle a m p P$ in PAO1. The resistance and muropeptide profile of these three mutants were compared against PAO1, respectively. A single deletion of ampG resulted in decreased resistance profile as compared with the wild-type PAO1, for many of the $\beta$ lactams as well as for fosfomycin. ampP deletion however, did not result in altering susceptibility. The profile of muropeptides was investigated by LC/MS/MS. The wholecell extracts across all the strains (wild-type and permease mutants) were examined for muropeptides and it was found that the relative levels of muropeptides were similar in PAO1 and PAO $\triangle a m p G$ deletion. The levels of disaccharide peptides in $\triangle a m p P$ and $\triangle a m p G \Delta a m p P$ were lower in the periplasm and the cytoplasm respectively, compared to PAO1. This is indicative of AmpP playing a role in muropeptide transport and supports our hypothesis. Periplasmic analysis of the muropeptides revealed that levels of monosaccharides were lower for all the strains in the periplasmic space as compared 
with whole-cells. This method of muropeptide detection is highly sensitive and allows us to compare the relative levels of muropeptides across bacterial strains.

\subsection{Introduction}

Muropeptides or cell-wall fragments are created whenever the bacterial cell divides. Gram-negative and Gram-positive pathogens have a dedicated recycling pathway that salvages these muropeptides and utilizes them during rebuilding of the cell-wall (Boothby, et al., 1973, Goodell, 1985). The muropeptides besides being recycled to conserve energy resources also play critical roles in bacterial survival and virulence. In Gram-positive pathogen Bacillus subtilis, Gram-negative bacteria Myxococcus xanthus and Micrococcus luteus, muropeptides trigger reactivation of dormant spores in response to environmental cues (Shimkets \& Kaiser, 1982, Mukamolova, et al., 2006, Shah, et al., 2008). In P. aeruginosa, production of a toxin, pyocyanin is modulated by peptidoglycan fragments containing $\mathrm{N}$-acetylglucosamine (Korgaonkar \& Whiteley, 2011). The muramyl peptides also have well defined role as stimulants of the host immune system (Takeuchi \& Akira, 2010). This immune response in a healthy individual is triggered partly by certain elements of the bacterial cell itself known as pathogen associated molecular patterns (PAMP) (Janeway, 1989). These include mainly the lipopolysaccharide, peptidoglycan, flagellin proteins and lipotechoic acid in Grampositive bacteria. These structures are recognized by the innate immune system, namely the Toll-like receptors (TLR) and the nucleotide-binding oligomerization domain (NOD) (Takeuchi \& Akira, 2010, Fujimoto \& Fukase, 2011). Peptidoglycans in specific are recognized by the NOD family- Nod 1, Nod 2, cryopyrin and the peptidoglycan response proteins (PGRP) (McDonald, et al., 2005, Fujimoto \& Fukase, 2011). Specifically, $\gamma-D$ 
glutamyl-meso-diaminopimelic acid muramyl dipepetides act as recognition moieties and activate the pro-inflammatory cytokines (Chamaillard, et al., 2003, Girardin, et al., 2003, Boudreau, et al., 2012). Due to the key role of muropeptides both within the bacteria as signaling components as well as in the eukaryotic host as an immune stimulus, identifying the muropeptides and the recycling process becomes significant in understanding bacterial activity and its interaction with host. The recycling pathway has evolved in many Gram-negative pathogens however it has been best studied in $E$. coli (Park \& Uehara, 2008, Johnson, et al., 2013, Dhar, et al., 2018).

During the cell wall recycling process in E. coli, PG fragments are generated in periplasm through the activity of lytic transglycosylases, penicillin binding proteins (PBP) and amidases (Holtje, et al., 1975, Park \& Uehara, 2008). PG fragments enter the cytoplasm through permease AmpG (Jacobs, et al., 1994). In the cytoplasm, they are initially processed by two enzymes, NagZ that removes the $N$-acetylglucosamine residue followed by AmpD which cleaves the peptide chain from the muropeptide (Yem \& Wu, 1976, Jacobs, et al., 1994, Jacobs, et al., 1995, Hesek, et al., 2009). The recycling process in $P$. aeruginosa has not been completely elucidated and there have been no studies that determine the muropeptide profile of $P$. aeruginosa. Bioinformatic analyses led to the identification of E. coli homologs of the recycling pathway (Kong, et al., 2010, Dhar, et al., 2018). However, there are differences in the recycling pathway in $P$. aeruginosa when compared with other members of Enterobacteriaceae. $P$. aeruginosa has periplasmic paralogs of AmpD known as AmpDh2 and AmpDh3 (Juan, et al., 2006, Lee, et al., 2013, Martinez-Caballero, et al., 2013, Zhang, et al., 2013). It also has a AmpG paralog, AmpP which we hypothesize is a second muropeptide transporter (Kong, 
et al., 2010). This part of the research addresses the role of the permeases AmpG and AmpP in $P$. aeruginosa with regards to cell-wall recycling in $P$. aeruginosa.

AmpG (PA4393): AmpG has been identified in 134 distinctive genera of bacteria including pathogens such as $P$. aeruginosa, Neisseria gonorrhea, Serratia marcescens, Vibrio cholerae, Acinetobacter baumanii and Salmonella species (Folkesson, et al., 2005, Garcia \& Dillard, 2008, Adin, et al., 2009, Li, et al., 2016). Most AmpG proteins were found to have 12-14 transmembrane (TM) domains ( $\mathrm{Li}$, et al., 2016). The crucial role of AmpG in $\beta$-lactam resistance was realized for the first time upon its identification in Enterobacter cloacae (Korfmann \& Sanders, 1989). P. aeruginosa AmpG has a particularly long sequence with 14 transmembrane spanning domains. A high degree of sequence conservation is observed amongst AmpG found in different bacteria. $A$ previous study showed that $P$. aeruginosa AmpG can be successfully complemented by AmpG from other Gram-negative pathogens such as Acinetobacter baumanii, Escherichia coli, and Vibrio cholera (Li, et al., 2016).

The vital role played by AmpG in muropeptide recycling modifies not just $\beta$-lactam resistance but also the pathogenicity. This has been the research focus regarding AmpG in Neisseria species (Garcia \& Dillard, 2008, Woodhams, et al., 2013). It was found that non-pathogenic strains of Neisseria sp. such as $N$. sicca and $N$. mucosa were more efficient at recycling their muropeptides (Chan \& Dillard, 2016). Furthermore, replacing AmpG of pathogens such as $N$. gonorrhea by its homolog from a human colonizer such as $N$. meningitides or with AmpG from E. coli decreased the virulence of the pathogen (Chan \& Dillard, 2016). The diminished pathogenicity after AmpG substitution was correlated with a reduced release of muropeptides and more efficient recycling (Garcia \& Dillard, 2008, Chan \& Dillard, 2016). Another example is Bordetella pertussis where the 
causative agent of whooping cough, the tracheal cytotoxin, is a muropeptide (Goldman \& Herwaldt, 1985, Luker, et al., 1993). Specifically, it is composed of N-acetylglucosamine 1, 6 anhydro N-acetylmuramic acid tetrapeptide (Goldman \& Herwaldt, 1985, Goldman \& Cookson, 1988). The release of this tracheal cytotoxin into the host was depleted when the B. pertussis ampG gene was replaced by that of E. coli (Feunou, et al., 2008). ampG is also one of the genes targeted during the construction of a live-attenuated vaccine for B. pertussis (Mielcarek, et al., 2006). This reflects once again upon the fact that pathogenic bacteria, unlike in non-pathogens often have an inefficient muropeptide recycling due to a deficient AmpG.

In $P$. aeruginosa, AmpG is encoded by $P A 4393$ and localized to the inner membrane and act as a permease for the muropeptides transport from periplasm into the cytoplasm (Kong, et al., 2010, Perley-Robertson, et al., 2016). Its role in $\beta$-lactam resistance was further proved when a decrease in MIC was noted upon the deletion of this gene (Kong, et al., 2010, Zhang, et al., 2010). This decrease was relevant not just in the lab reference strain PAO1, but in clinical strains as well wherein attenuating AmpG resulted in restoring the MIC to below CLSI breakpoints by blocking the expression of AmpC (Zamorano, et al., 2011). Besides P. aeruginosa, the importance of AmpG as an activator of AmpC expression and resistance has been examined in Xanthomonas campestris, Strenotophomonas maltophila, Enterobacter cloacae, Citrobacter freundii (Korfmann \& Sanders, 1989, Lindquist, et al., 1993, Huang, et al., 2010, Yang, et al., 2013).

AmpP (PA4218): P. aeruginosa PA4218 encodes for a protein that was shown to be involved in $\beta$-lactam resistance in $P$. aeruginosa (Kong, et al., 2010). Previous research in the lab showed that inactivating AmpP resulted in a sub-maximal $\beta$-lactamase 
expression (Kong, et al., 2010). This phenotype was restored to that of wild-type by intrans ampP complementation. This indicated to us that AmpP plays a role in $\beta$-lactam resistance. Protein structure analysis also indicated that this is a permease with a Major Facilitator Superfamily domain. Localization studies using LacZ-PhoA fusions found that it lies in the inner membrane with ten TM spanning regions (Kong, et al., 2010). Its role in regulating $\beta$-lactamase expression along with its activity as a permease and significant homology with $P$. aeruginosa AmpG led to the hypothesis that this protein may be involved in the cell-wall recycling pathway. AmpP is the second gene of a two-gene operon whose expression is found to be controlled by a transcriptional regulator AmpR independent of any $\beta$-lactam induction (Kong, et al., 2010, Balasubramanian, et al., 2012). Besides the research in our lab, AmpP was also found to be involved in pyochelin utilization which is one of the two siderophores found in $P$. aeruginosa (Michel, et al., 2007, Cunrath, et al., 2015).

Although it has been known that AmpG acts as a muropeptide transporter in $P$. aeruginosa, its specific role in cell-wall recycling has not been studied. Similarly, the role of AmpP in cell-wall recycling has not been explored. Thus, this research addresses the gap in knowledge regarding the function of these inner membrane permeases in cell-wall recycling. This study will compare the accumulation of the muropeptides in the parent reference strain $\mathrm{PAO} 1$ with its isogenic permease mutants.

\subsection{Materials and Methods}

3.3.1 Strains, media and growth conditions. Pseudomonas aeruginosa PAO1 and its derivatives (PAO $\triangle a m p G, \mathrm{PAO} \triangle a m p P, \mathrm{PAO} \Delta a m p G \Delta a m p P)$ and Escherichia coli $\mathrm{DH} 5 \alpha$ 
were used in this study. For in vivo homologous recombination, the yeast model Saccharomyces cerevisiae InvSC1 strain (Invitrogen ${ }^{\text {TM }}$, Life Technologies, Carlsbad, CA, USA) was used (Shanks, et al., 2006). E. coli and P. aeruginosa were cultured in Luria Bertani broth (5 $\mathrm{g}$ tryptone, $10 \mathrm{~g}$ sodium chloride, and $5 \mathrm{~g}$ yeast extract per liter) with supplements of $1.5 \%$ agar when needed at $37^{\circ} \mathrm{C}$. For triparental mating experiments, Pseudomonas Isolation Agar (Difco) was used along with LB for P. aeruginosa selection. S. cerevisiae was cultured in YEPED media (Yeast extract-peptone-dextrose media: 20 $\mathrm{g}$ Bacto Peptone, $10 \mathrm{~g}$ yeast extract, and $20 \mathrm{~g}$ dextrose per liter) at $30{ }^{\circ} \mathrm{C}$. Selection of yeast colonies with transformed pMQ30 was done using synthetic define agar-uracil media. For plasmid selection and maintenance, antibiotics at the following concentrations were used: gentamicin $(\mathrm{Gm}) 15 \mu \mathrm{g} / \mathrm{mL}$ for $E$. coli, $75 \mu \mathrm{g} / \mathrm{mL}$ for $P$. aeruginosa, chloramphenicol $(\mathrm{Cm}) 10 \mu \mathrm{g} / \mathrm{mL}$ and kanamycin $(\mathrm{Km}) 20 \mu \mathrm{g} / \mathrm{mL}$ for E. coli.

\subsubsection{Construction of ampG and ampP deletion mutants by homologous} recombination. The deletions were constructed in Pseudomonas aeruginosa PAO1 genomic background (Holloway \& Morgan, 1986). Upstream and downstream regions of ampG (PA4393) were amplified to generate what we refer to as $\mathrm{P} 1$ and $\mathrm{P} 2$. The P1 and P2 were produced by PCR using primers pairs LS_PA4393 UF, LS_PA4393 UR and LS_PA4393DF, LS_PA4393DR that gave amplicons of 986-bp and 991-bp, respectively. Similarly, for ampP (PA4218), primer pairs LS_PA4218 UF, LS_PA4218 UR and LS_PA4218 DF, LS_PA4218 DR were used to generate 997-bp and 926-bp P1 and P2, respectively. The amplified fragments were checked by PCR and its fidelity was determined by sequencing. The $\mathrm{P} 1, \mathrm{P} 2$ of $a m p G$ and $a m p P$ were transformed into Saccharomyces cerevisiae respectively, with a linearized (BamH1 digested) vector 
pMQ30 to generate the spliced product (P3) (Shanks, et al., 2006). PCR and agarose gel electrophoresis were used to screen the yeast colonies for the presence of the P3 following which yeast DNA was isolated from the positive clones. The recombinant construct was extracted from yeast and introduced in E. coli. The positive colonies were selected by their growth on gentamicin and the presence and fidelity of the P3 with the deleted target gene was checked by PCR and sequencing. The P3 constructs was conjugated into $P$. aeruginosa PAO1 independently by triparental mating using E.colipRK2013 and pRK600 as helper strains (Figurski \& Helinski, 1979, Finan, et al., 1986). The single and double crossover products were confirmed by Gm sensitivity followed by selection for loss of pMQ30 plasmid through sucrose resistance (Oldenburg, et al., 1997, Shanks, et al., 2006). The integration of the DNA fragment in the chromosome and the loss of the target genes were verified by PCR and sequencing. The ampP deletion (plasmid with P3 fragment) was also introduced in the ampG deletion to create the permease double deletion mutant, PAO $\triangle a m p G \Delta a m p P$.

Table 3. 1: Strains, plasmids and primers used in this study.

\begin{tabular}{|c|c|c|}
\hline Strains & Relevant phenotype and genotype & $\begin{array}{l}\text { Reference/ } \\
\text { Source }\end{array}$ \\
\hline \multicolumn{3}{|l|}{ Escherichia coli } \\
\hline $\mathrm{DH} 5 \alpha$ & $\begin{array}{l}\text { F- } \Phi 80 \text { lacZLM15 } \Delta(\text { lacZYA argF)U169 deoR } \\
\text { recA1 endA1 hsdR17 (rk-mk+) phoA supE44 } \lambda^{-}- \\
\text {thi-1 gyrA96 relA1 }\end{array}$ & $\begin{array}{l}\text { New } \\
\text { England } \\
\text { Biolabs }\end{array}$ \\
\hline \multicolumn{3}{|c|}{ Saccharomyces cerevisiae } \\
\hline INVSc1 & $\begin{array}{l}\text { MATa/MATa leu2/leu2 trp1-289/trp1-289 ura3- } \\
\text { 52/ura3-52 his3- } \Delta 1 / \text { his3- } \Delta 1\end{array}$ & Invitrogen \\
\hline \multicolumn{3}{|c|}{ Pseudomonas aeruginosa } \\
\hline PAO1 & Wild-type & $\begin{array}{l}\text { (Holloway, } \\
\text { et al., 1979) }\end{array}$ \\
\hline PAO $\triangle a m p G$ & PAO1 contain & This study \\
\hline PAO $\triangle a m p P$ & PAO1 containing in-frame deletion of $a m p P$ & This study \\
\hline $\mathrm{PAO} \triangle a m p G \Delta a m p P$ & $\begin{array}{l}\text { PAO1 containing in-frame deletions of } a m p G \text { and } \\
a m p P\end{array}$ & This study \\
\hline $\mathrm{PAO} \triangle a m p G(\mathrm{pAmpG})$ & $\begin{array}{l}\text { PAO } \triangle a m p G \text { containing ampG on plasmid } \\
\text { pMQ72 }\end{array}$ & This study \\
\hline
\end{tabular}




\begin{tabular}{|c|c|c|}
\hline PAO $\triangle a m p P(p A m p P)$ & PAO $\triangle a m p P$ containing $a m p P$ on plasmid pMQ72 & This study \\
\hline \multicolumn{3}{|l|}{ Plasmids } \\
\hline pGEMT & $\begin{array}{l}\text { TA cloning vector for PCR products; } \mathrm{Ap}^{R} \text {, } \\
\text { ColE1 f1 ori lacZ } \alpha\end{array}$ & Promega \\
\hline pRK600 & $\begin{array}{l}\mathrm{Cm}^{\mathrm{R}} ; \text { colE1 tra }{ }^{+} \mathrm{RK} 2 \mathrm{mob}^{+} ; \text {Helper plasmid for } \\
\text { conjugation }\end{array}$ & $\begin{array}{l}\text { (Figurski \& } \\
\text { Helinski, } \\
\text { 1979) }\end{array}$ \\
\hline pRK2013 & $\begin{array}{l}\mathrm{Km}^{\mathrm{R}} \text {; colE1 tra+ RK2 } \mathrm{mob}^{+} \text {; Helper plasmid for } \\
\text { conjugation }\end{array}$ & $\begin{array}{l}\text { (Finan, et } \\
\text { al., 1986) }\end{array}$ \\
\hline pMQ30 & $\mathrm{Gm}^{\mathrm{R}}$; colE1, oriT & $\begin{array}{l}\text { (Shanks, et } \\
\text { al., 2006) }\end{array}$ \\
\hline pSD51 & $\begin{array}{l}\mathrm{Ap}^{\mathrm{R}} ; \mathrm{A} \sim 1.8-\mathrm{kb} \text { Nhel-Sacl fragment containing } \\
\text { ampG ORF (PA4393) amplified from PAO1 } \\
\text { genome using SD_ampG_FW and } \\
\text { SD_ampG_Rev primers and cloned into pGEMT }\end{array}$ & This study \\
\hline pSD57 & $\begin{array}{l}\mathrm{Ap}^{\mathrm{R}} ; \mathrm{A} \sim 1.2-\mathrm{kb} \text { Nhel-Sacl fragment containing } \\
\text { ampP ORF (PA4218) amplified from PAO1 } \\
\text { genome using SD_ampP_FW and } \\
\text { SD_ampP_Rev primers and cloned into pGEMT }\end{array}$ & This study \\
\hline pSD55 & $\begin{array}{l}\mathrm{Gm} \text {; } \\
\text { an Nhel-Sacl fragment into pMQ72 }\end{array}$ & This study \\
\hline pSD61 & $\begin{array}{l}\mathrm{Gm}^{\mathrm{R}} ; \text { The ampP ORF sub-cloned from pSD as } \\
\text { an Nhel-Sacl fragment into pMQ72 }\end{array}$ & This study \\
\hline \multicolumn{3}{|c|}{ 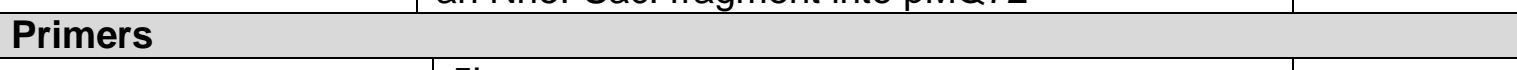 } \\
\hline LS_PA4393 UF & $\begin{array}{l}\text { 5'- } \\
\text { GGAATTGTGAGCGGATAACAATTTCACACAG } \\
\text { GAAACAGCTCGAAGAAGCGATCGAGGTCG- } \\
\text { 3' }\end{array}$ & This study \\
\hline LS_PA4393 UR & $\begin{array}{l}\text { 5'- } \\
\text { CGAATGCCGGCCTTTTTCATTCTCGCCTAGC } \\
\text { TAGCTAGGGGTAACCGTTCCGCAAAA-3' }\end{array}$ & This study \\
\hline LS_PA4393 DF & $\begin{array}{l}\text { 5'- } \\
\text { TTTTGCGGAACGGTTACCCCTAGCTAGCTAG } \\
\text { GCGAGAATGAAAAAGGCCGGCATTCG-3' }\end{array}$ & This study \\
\hline LS_PA4393 DR & $\begin{array}{l}\text { 5'- } \\
\text { CCAGGCAAATTCTGTTTTATCAGACCGCTTC } \\
\text { TGCGTTCTGATGGAAACGTCGCCCCACGG- } \\
\text { 3' }\end{array}$ & This study \\
\hline LS_PA4218 UF & $\begin{array}{l}\text { 5'- } \\
\text { GGAATTGTGAGCGGATAACAATTTCACACAG } \\
\text { GAAACAGCTGACCTGCATGGCCTGATCGG- } \\
\text { 3' }\end{array}$ & This study \\
\hline LS_PA4218 new UR & $\begin{array}{l}\text { 5'- } \\
\text { GCCTATCGGCAGCGCCTCACGCTAGCTCAA } \\
\text { GCATGGTGGTCTCCGGTGAC-3' }\end{array}$ & This study \\
\hline LS_PA4218 new DF & $\begin{array}{l}\text { 5'- } \\
\text { GTCACCGGAGACCACCATGCTTGAGCTAGC } \\
\text { GTGAGGCGCTGCCGATAGGC-3' }\end{array}$ & This study \\
\hline LS_PA4218 DR & 5 & This study \\
\hline
\end{tabular}




\begin{tabular}{|l|l|l|}
\hline & $\begin{array}{l}\text { CCAGGCAAATTCTGTTTTATCAGACCGCTTC } \\
\text { TGCGTTCTGATCTCGGCGTGGAGCGTATC-3' }\end{array}$ & \\
\hline SD_PA4218_Fw_Nhe1 & $\begin{array}{l}\text { 5'- } \\
\text { GGGCTAGCAGGAGAGACCTCACCCTGATCC } \\
\text { TCT-3' }\end{array}$ & This study \\
\hline $\begin{array}{l}\text { SD_PA4218_Rev_Sac } \\
1\end{array}$ & $\begin{array}{l}\text { 5'- } \\
\text { GGGAGCTCGCGGGAGAGGCGGCATCCGT- } \\
\text { 3' }\end{array}$ & This study \\
\hline SD_PA4393_Fw_Nhe1 & $\begin{array}{l}\text { 5'- } \\
\text { GGGCTAGCAGGAGATTGCGCGAGGAAGGC } \\
\text { GTTCT-3' }\end{array}$ & This study \\
\hline SD_PA4393_Rev_Sac & $\begin{array}{l}\text { 5'- } \\
\text { GGGAGCTCGCGATGGCCGACTGAGGTCCG } \\
\text { 1 }\end{array}$ & This study \\
\hline
\end{tabular}

3.3.3 Construction of complementing plasmids. DNA fragments from $P$. aeruginosa PAO1 with ampG $(\sim 1.78 \mathrm{~kb})$ and $a m p P(\sim 1.2 \mathrm{~kb})$ were PCR amplified using primer pairs SD_PA4393_Fw_Nhe1 and SD_PA4393_Rev_Sac1 and SD_PA4218_Fw_Nhe1 and SD_PA4218_Rev_Sac1, respectively (Table 2.1). The PCR amplified products were cloned into pGEMT (Promega Corporation, USA) using manufacturers protocol to generate plasmids PSD51 and PSD57, respectively. The fidelity of the PCR amplified product was confirmed by sequencing. The fragments carrying ampG and ampP were moved into a broad host range pMQ72 plasmid as Nhel-Sacl fragments, downstream of an inducible $\mathrm{P}_{\text {lacuv5 }}$ promoter to generate plasmids pSD55 and pSD62, respectively. Henceforth, these plasmids are referred to as pAmpG and pAmpP. These expression plasmids were then introduced into wild-type PAO1, PAO $\triangle a m p G$, and PAO $\triangle a m p P$ deletion mutants by electroporation and gentamycin resistant colonies were selected.

3.3.4 Growth curves. $P$. aeruginosa PAO1 and its derivatives were grown overnight at $37^{\circ} \mathrm{C}$ in LB broth with or without antibiotics. Overnight cultures were washed with sterile $0.85 \% \mathrm{NaCl}(\mathrm{wt} / \mathrm{vol})$ solution to remove spent media. Cultures were diluted in fresh LB broth to obtain equal optical densities $\left(\mathrm{OD}_{600}\right)$ of 1 . From this culture, they were diluted 
again to a starting $\mathrm{OD}_{600}$ of 0.05 . Growth was monitored by determining absorbance at $600 \mathrm{~nm}$ using BioTek Synergy HT (Winooski, VT, USA) plate reader for $16-18 \mathrm{~h}$ at $37^{\circ} \mathrm{C}$.

3.3.5 Determination of minimum inhibitory concentration (MIC). The MIC was determined using the E-test (BioMerieux) (Melvin P. Weinstein, 2018). Briefly, overnight cultures of bacteria were diluted to an OD of 0.02-0.2 and spread on an LB-agar plate. An E-strip which contains antibiotics at an increasing concentration along its length was placed on the plate. The MIC was determined by the zone of clearance on the plate after 16-18 hr incubation at $37^{\circ} \mathrm{C}$.

3.3.6 Quantifying $\beta$-lactamase activity. $\beta$-lactamase activity was quantified as described previously (O'Callaghan, et al., 1972, Kong, et al., 2005). Briefly, cells in LB broth at an OD600 of $0.5-0.6$ were treated with $100 \mu \mathrm{g} / \mathrm{ml}$ Penicillin $\mathrm{G}$ for two hours at 37 ${ }^{\circ} \mathrm{C}$. The cells were then harvested, OD normalized, and lysed with BugBuster Protein Extraction Reagent (Novagen, WI) and r-Lysozyme (Novagen, WI) and treated with Benzonase nuclease (Novagen,WI). The amount of $\beta$-lactamase was quantified in the soluble fraction by determining hydrolyzing activity on nitrocefin (Oxoid, England). Protein concentrations in the samples were determined by Bradford assay. Enzyme activity was expressed as milliunits of $\beta$-lactamase (nanomoles of chromocef hydrolyzed per minute per microgram of protein).

3.3.7 Release of muramyl peptides from whole cell extracts. $P$. aeruginosa (wild type; PAO1 and mutants; PAO $\triangle a m p G, \mathrm{PAO} \triangle a m p P$ ) was grown until late log phase. The culture was harvested by centrifugation at $11,000 \mathrm{~g}$ for $20 \mathrm{~min}$ and the supernatant discarded. The cell pellet was resuspended gently in cold shrinking solution $(20 \%$ sucrose, $1.2 \mathrm{mM}$ EDTA, $30 \mathrm{mM}$ Tris, $\mathrm{pH}$ 8) and left with shaking at $4^{\circ} \mathrm{C}$ for $10 \mathrm{~min}$. This 
was centrifuged followed by resuspension of the pellet in ice cold water. The suspension was kept at $4^{\circ} \mathrm{C}$ for $10 \mathrm{~min}$, and then centrifuged at $11,000 \mathrm{~g}$ for $20 \mathrm{~min}$ and the supernatant discarded. The pellet was then boiled to quench enzyme activity and sonicated to ensure complete release of muropeptides. The sample was filtered using 30 KDa molecular weight cut off mini-column. The remaining solution was concentrated through speed vacuum at $50^{\circ} \mathrm{C}$ and used for LC/MS analysis. Dionex Ultimate 3000 Rapid Separation UPLC system coupled to Bruker MicrOTOF-Q II Mass spectrometer was used for the analysis (Lee, et al., 2013).

3.3.8 Release of muramyl peptides from periplasm. Periplasmic extraction was done using osmotic shock, as previously described with minor modifications (Nossal \& Heppel, 1966). The culture was grown until $\mathrm{OD}_{600}$ 1.2. The bacteria was harvested and suspended in buffer containing Tris- $\mathrm{Cl}(\mathrm{pH}-7.4)$ and magnesium chloride $200 \mathrm{mM}$. This suspension was kept under rotation for 15 minutes at room temperature and reharvested again. The culture was then re-suspended in ice-cold water and kept under shaking at $4{ }^{\circ} \mathrm{C}$. The culture was harvested again, and the supernatant collected. This contains the periplasmic proteins. For our analysis by LC/MS, this was filtered through 30KDa mini-column and the filtrate was collected. The filtrate was boiled to quench any enzyme activity and concentrated using a speed-vacuum. This sample was analyzed by LC/MS.

\subsubsection{Detection of muropeptides by liquid chromatography/mass spectrometry.} The conditions used for LC/MS have been previously developed for muropeptide analysis (Lee, et al., 2013). Briefly, the separation was done using a Dionex Ultimate 3000 Rapid Separation UPLC system equipped with a Dionex Ultimate 3000 autosampler and a Dionex Ultimate 3000 photodiode array detector. Separations were 
performed on a Dionex Acclaim ${ }^{\text {TM }}$ PolarAdvantage II C18 column $(3 \mu \mathrm{m}, 120 \AA$, $2.1 \mathrm{~mm}$ i.d. $\times 150 \mathrm{~mm}$ ). The mobile phase consisted of $A=0.1 \%$ formic acid in water; $B=0.1 \%$ formic acid in acetonitrile. This was coupled with a Bruker MicrOTOF-Q II quadrupole time-of-flight hybrid mass spectrometer. The Bruker electrospray ionization source was operated in the positive ion mode.

3.3.10 Statistical analyses. All data were analyzed for statistical significance using the Student's t-test on GraphPad or Analysis of Variance (ANOVA) with post-hoc testing when appropriate.

\subsection{Results and Discussion}

This study was done by extracting cell-lysates during log growth phase of bacteria and determining the relative levels of individual muropeptide. Instead of radioactivity which was previously used to study muropeptide levels, a liquid chromatography/massspectrometry approach is utilized here. This allowed us to identify muropeptides at the micro molar concentrations as well as to pin-point subtle changes in the profile of PG fragments between different strains. This approach of muropeptide analysis was however only possible as synthetic muropeptides were available (Hesek, et al., 2009).

\subsubsection{Growth analysis of mutant strains. The PAO $\triangle a m p G, P A O \triangle a m p P$ and} PAO $\triangle a m p G \Delta a m p P$ mutants exhibited no discernible phenotype in growth compared to the parent PAO1 when tested in LB media over 16-18 hours (Figure 3.1). This demonstrates that the permeases in cell-wall recycling are not essential for growth in $P$. aeruginosa. 


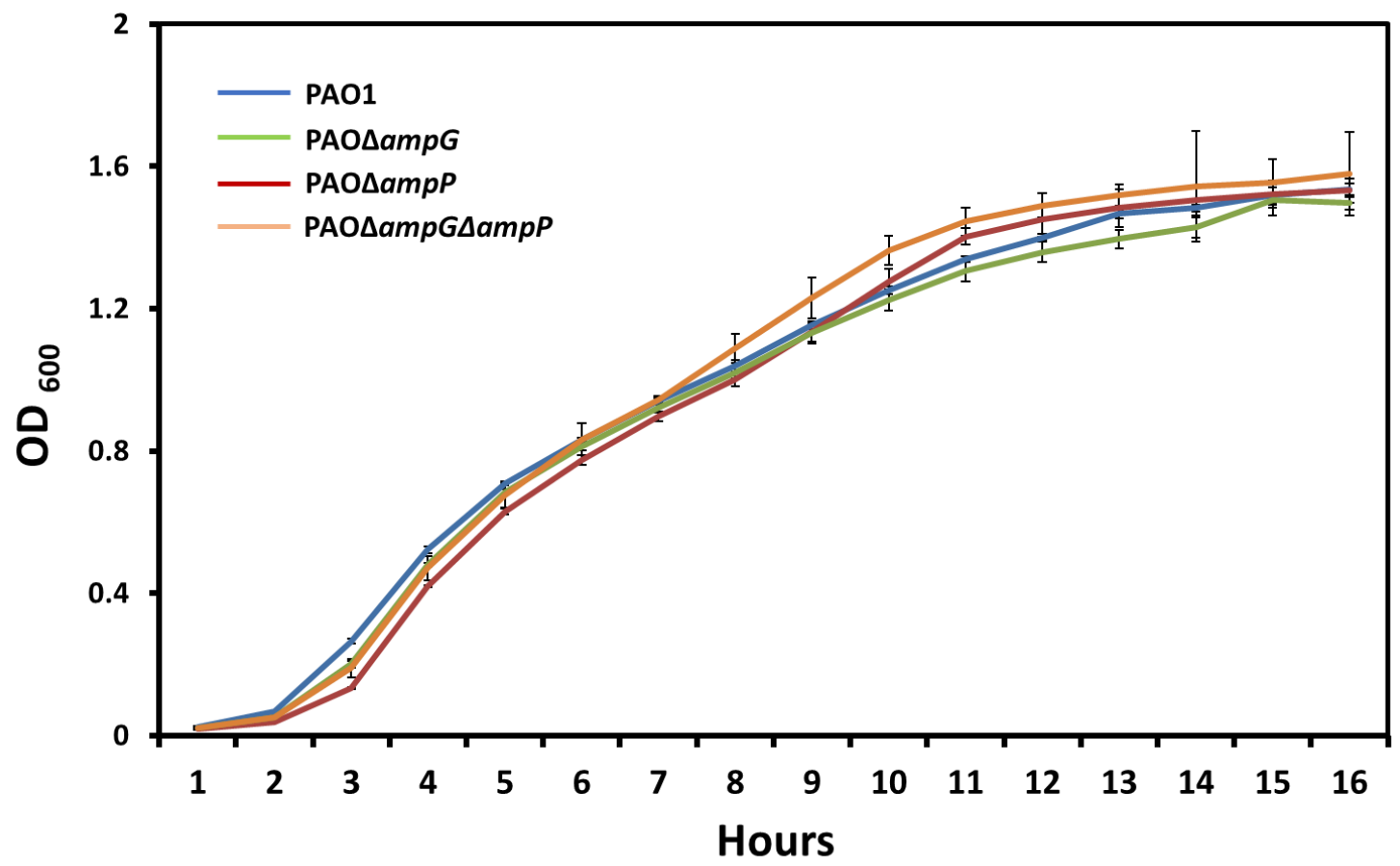

Figure 3. 1. Growth curve analysis of mutants. Growth curves of PAO1 and the mutants PAO $\triangle a m p G, \mathrm{PAO} \triangle a m p P$ and PAO $\triangle a m p G \Delta a m p P$ was analyzed in LB broth at $37^{\circ} \mathrm{C}$ over 16 hours. No difference was seen in the growth of mutants in this media.

3.4.2 MIC of $\beta$-lactams is altered in ampG deletion. To investigate the role of ampG in $P$. aeruginosa, precise in-frame deletion of the gene was generated. The susceptibility of selected antibiotics was compared with that of the parent strain PAO1. Antibiotics were chosen based upon two criteria. This included firstly the antibiotics which are predominantly used in the clinical setting in case of a multi-drug resistant infection. These include a combination therapy with aminoglycosides, fluoroquinolones, and fosfomycin (Samonis, et al., 2012, Tangden, 2014). Secondly, as the role of ampG in resistance is manifested through cell-wall recycling and $A m p R$, the antibiotics that have 
previously shown a differential MIC in PAO $\triangle a m p R$ were selected (Balasubramanian, et al., 2013).

The MIC for the $\beta$-lactams which include aminopenicillins (ampicillin) cephalosporins (ceftazidime and cefoxitin) and carbapenems were reduced in the absence of ampG when compared with the wild-type PAO1. This phenotype was restored to the wild-type levels upon in-trans complementation. However, the MIC of ceftazidime which is a weak inducer of AmpC was unaltered. Interestingly, the MIC to one of the last-resort antibiotics for resistant infection, fosfomycin, was also decreased in PAO $\triangle a m p G$ (Table 3.1).

3.4.3 $\beta$-lactamase expression is reduced in ampG deletion mutants. Previously, a study in our lab had shown that deletion of ampG resulted in the production of submaximal levels of $\beta$-lactamase (Kong, et al., 2010). As an insertional inactivation mutant had been used in the earlier study, we verified our observations with a precise deletion. Upon comparing the results with PAO1, it was found that PAO $\triangle \mathrm{ampG}$ had significantly $(p<0.05)$ decreased levels of $\beta$-lactamase expression. 
Table 3.1. Susceptibility profiles of PAO $\triangle a m p G, P A O \triangle a m p P$ and $P A O \Delta a m p G \Delta a m p P$.

\begin{tabular}{|c|c|c|c|c|c|c|}
\hline \multirow[b]{2}{*}{ Antibiotics } & \multicolumn{5}{|c|}{ Minimum Inhibitory Concentration ( $\mu \mathrm{g} / \mathrm{ml})$} & \multirow[b]{2}{*}{$\mathrm{PAO} \Delta a m p G \Delta a m p P$} \\
\hline & PAO1 & $\mathrm{PAO} \triangle a m p G$ & PAO $\triangle a m p P$ & $\mathrm{PAO} \triangle a m p G(p A m p G)$ & $\mathrm{PAO} \triangle a m p P(p A m p P)$ & \\
\hline Ampicillin & $>256$ & $32-64^{*}$ & $>256$ & $>256$ & $>256$ & $16-32^{*}$ \\
\hline Ceftazidime & 1.5 & 1.5 & $2-1.5$ & 2 & 1.5 & 2 \\
\hline Cefoxitin & $>256$ & $64^{*}$ & $>256$ & $>256$ & $>256$ & $64^{*}$ \\
\hline Imipenem & $2-1.5$ & $.38-.5^{*}$ & 2 & 2 & 1.5 & $.38^{*}$ \\
\hline Doripenem & 0.25 & 0.19 & 0.19 & 0.125 & 0.125 & .25 \\
\hline Meropenem & 0.38 & $0.38-0.5$ & 0.25 & $0.38-0.5$ & $0.38-0.5$ & .5 \\
\hline Ciprofloxacin & $0.25-0.5$ & 0.25 & 0.25 & 0.25 & 0.5 & .19 \\
\hline Ofloxacin & 2 & 1 & 1.5 & 1.5 & 2 & 1 \\
\hline Fosfomycin & 32 & $8^{*}$ & 32 & $24-16$ & 16 & $8-16$ \\
\hline Colistin & 0.75 & 0.75 & 0.75 & 0.75 & 1.5 & $0.75-1.5$ \\
\hline Trim-sox & 4 & 3 & 6 & 4 & 3 & 4 \\
\hline
\end{tabular}

*Significance was noted using student's T-test (PAO1 vs mutants, $p<0.05)$ 
3.4.4 MIC and $\beta$-lactamase expression is unaltered in ampP deletion. To investigate the role of ampP in $P$. aeruginosa, a precise in-frame deletion of the gene was generated. Previously, a study had shown that upon insertional inactivation of ampP, the $\beta$-lactamase expression was decreased (Kong, et al., 2010). However, no change was found in the MIC of $\beta$-lactams (Table 2.1). For induction of $\beta$-lactamase similar conditions as that for ampG strains were used. No significant differences were found in the $\beta$-lactamase expression levels of ampP deletion when compared with the wild-type PAO1.

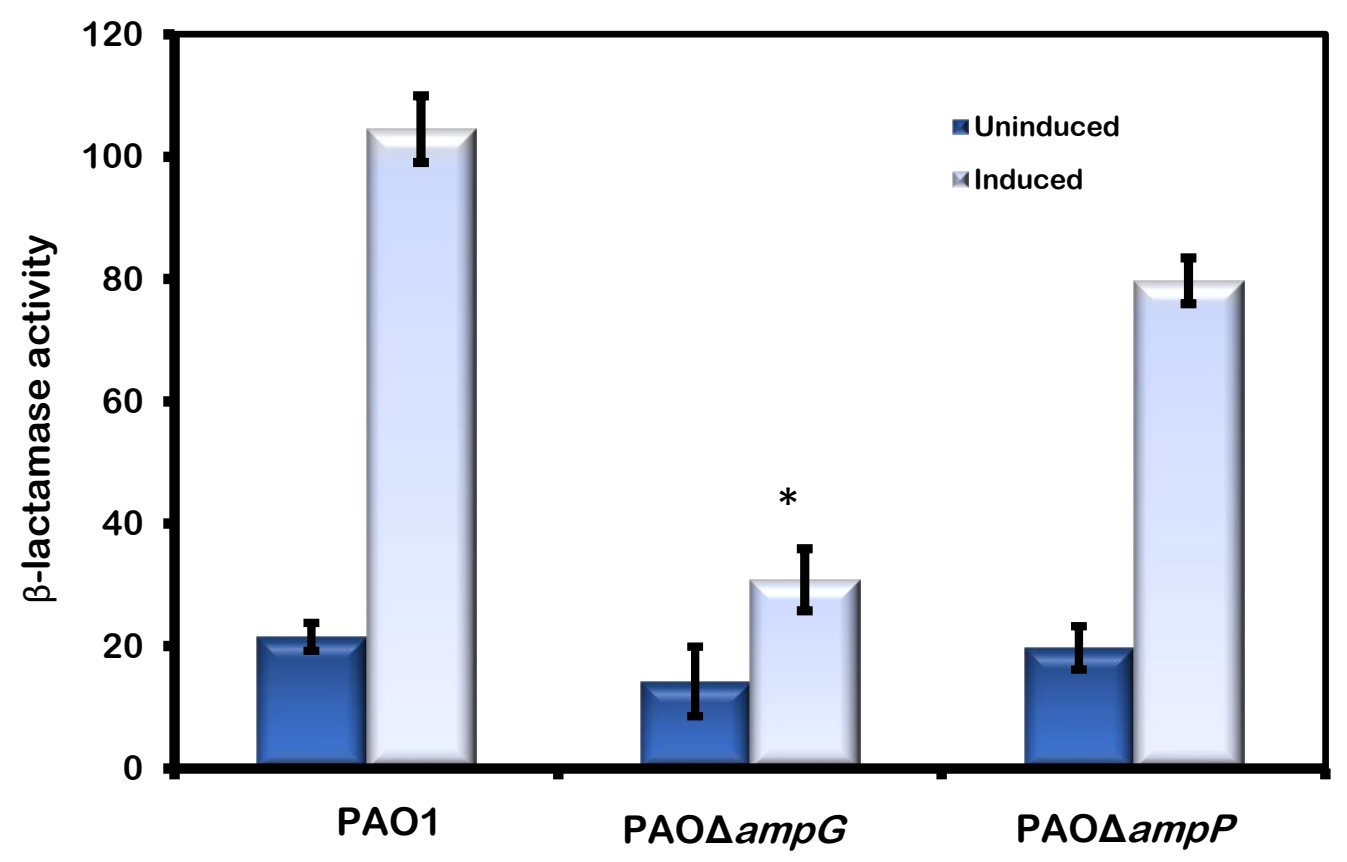

Figure 3.2. $\beta$-lactamase expression of $P A O I$ and permease mutants. The $\beta$-lactamase activity was quantified for PAO1, PAO $\triangle a m p G$, and PAO $\triangle a m p P$ in the presence and absence of a $\beta$-lactam (Penicillin 100ug $/ \mathrm{ml}$ ). ${ }^{*} p$-value $=0.004$ for $\beta$-lactamase activity in PAO $\triangle a m p G$ vs PAO1 induced, as determined by unpaired t-test. 
A.

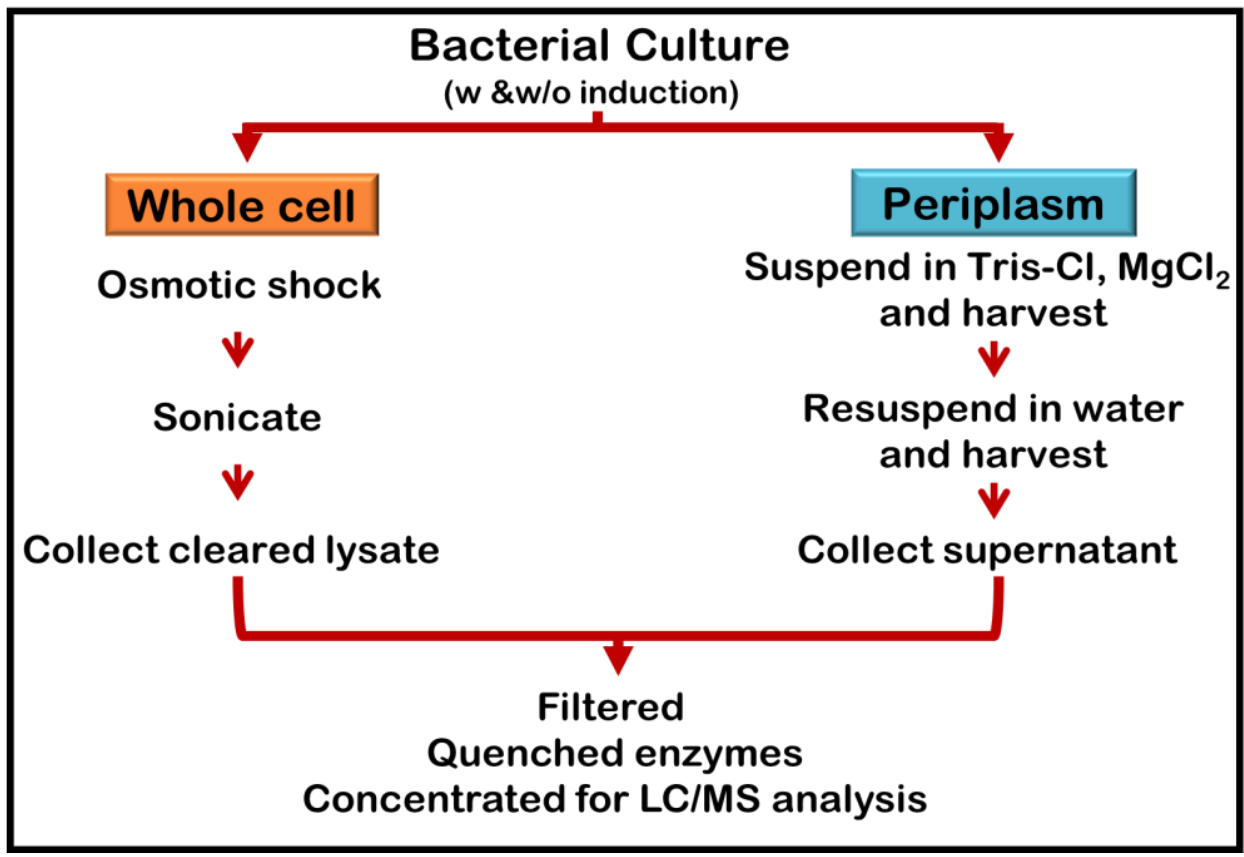

B.

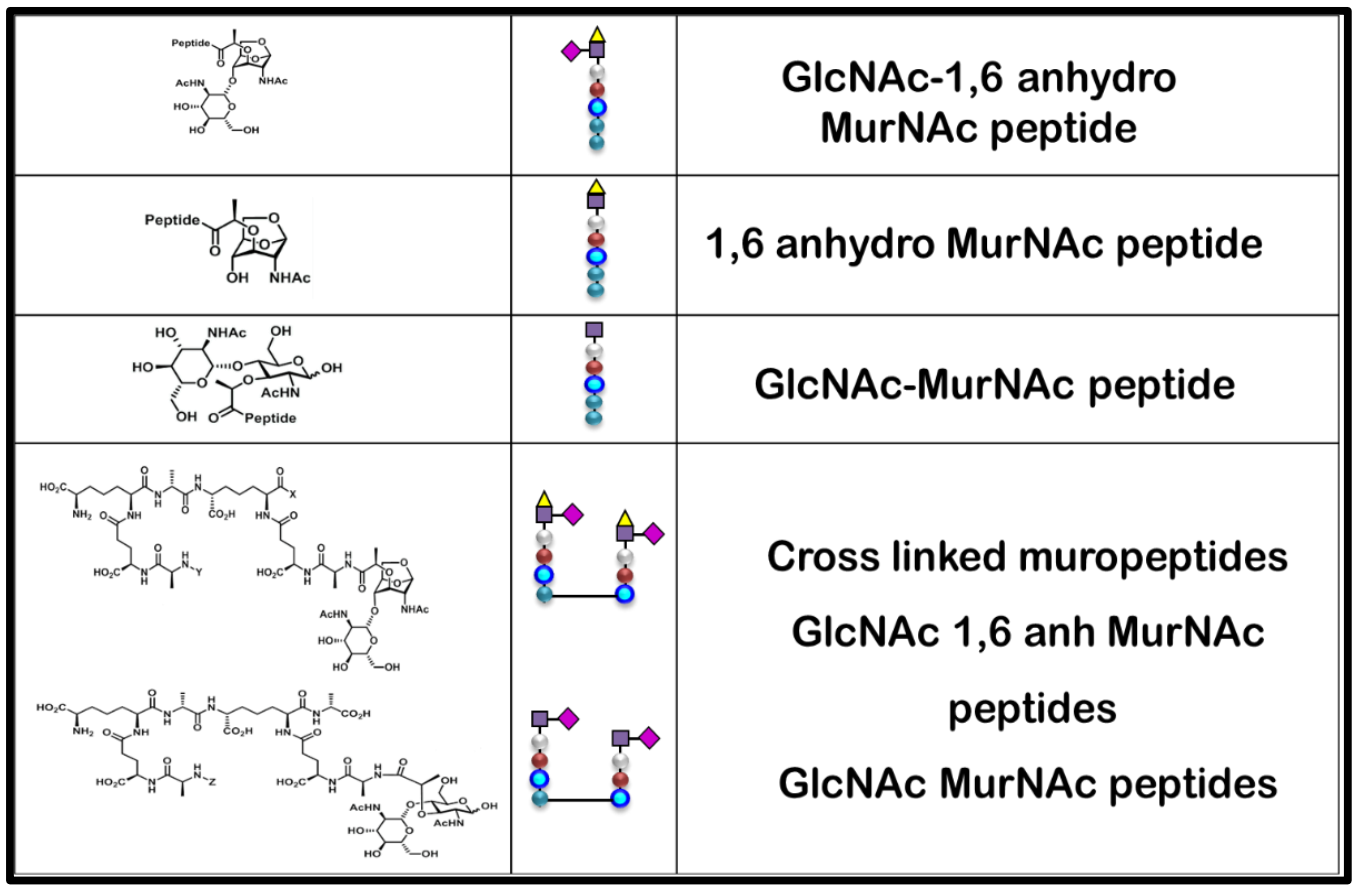

Figure 3.3. (A) A brief scheme of the muropeptide extraction protocol. (B) The structures of muropeptides. As found in $P$. aeruginosa as per our previous study are listed here (Lee, et al., 2016). 
3.4.5 Muropeptide profile of whole-cell extract in mutant strains. In this study, we compare the trend and profile of muropeptides in the permease mutant strains with the parent wild-type PAO1. We used LC/MS was used to qualitatively analyze the muropeptides from the bacterial lysate that was extracted during the late-log phase of growth. Standards included synthesized muropeptides and were run with every set of samples to ensure the LC column sensitivity and specificity (Figure 3.3). The trend of individual and total muropeptides were compared between ampG, ampP and the double permease deletion; PAO $\triangle a m p G \triangle a m p P$ with the wild-type PAO1.

It was found that across the mutant strains, the relative levels of a group of muropeptide such as disaccharide-peptide, monosaccharide peptides and larger muropeptides which include tetra-saccharide and the cross-linked peptides were not significantly different (Figure 3.4). Besides ampG, we also studied the muropeptide levels in ampP deletion mutant. Given its homology to E. coli AmpG (41\%), we hypothesize that this permease acts as a muropeptide transporter, possibly for the processed muropeptides from the cytoplasm to the periplasm (Kong, et al., 2010). When compared with the wild-type PAO1 and ampG deletion, it showed a lower relative level of disaccharide-peptides and a higher level of monosaccharide-peptides. The levels of the larger saccharide including the cross-linked are similar in both the strains (Figure 3.4).

The profile of muropeptide in the double deletion PAO $\triangle a m p G \Delta a m p$ mutant was similar to ampP deletion. The relative levels of disaccharide were low when compared with the PAO1. The levels of monosaccharide muropeptides were higher than PAO1 and similar to that of ampP deletion (Figure 3.4). 


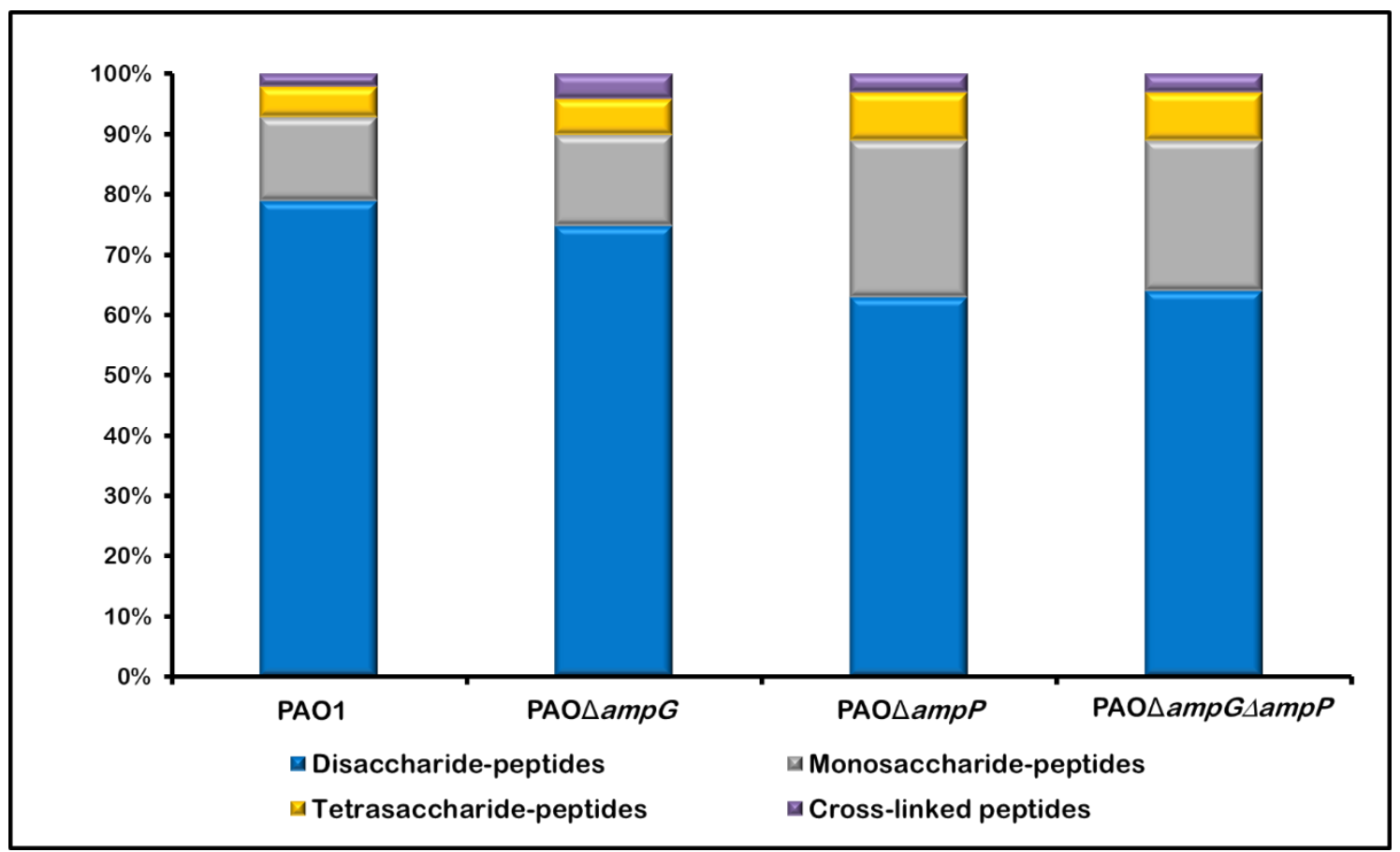

Figure 3. 4. Relative levels of muropeptides in whole-cells. The muropeptides extracted from whole cells. The area of individual muropeptides was calculated using area- under-the curve. They were grouped according to their saccharide backbone and compared across the strains. The disaccharides-peptide ranged from 0-4 amino acids i.e. no peptide to tetrapeptide moieties were found. Monosaccharide peptides include tri-and tetrapeptides. Tetrasaccharide peptides included tri and tetrapeptides. Crosslinked peptides detected were tri- and tetrapeptides, with reduced ends.

3.4.6 Muropeptide profile of periplasmic extract in mutant strains. As AmpG is a permease that localizes to the inner membrane and imports muropeptides from the periplasm to the cytoplasm, the profile of muropeptides in the periplasm was analyzed. The expectation was that most of the muropeptides would be unprocessed by cytoplasmic enzymes. In accordance with this most of the muropeptides in the periplasm were disaccharides (Figure 3.5). The major products in all the strains for the periplasmic cell-extract were the GlcNAc 1, 6 anhydroMurNAc without any peptides. This could be due to the action of the periplasmic AmpDh2 and AmpDh3 amidases which cleave the 
peptide chain. Although these enzymes have been noted to favor the full-sacculus, they also retain activity on the PG fragments (Zhang, et al., 2013). There was no significant difference between the individual muropeptides on comparing the single permease deletion mutant strains PAO $\triangle a m p G$ or PAO $\triangle a m p P$ with PAO1 (Figure 3.5). However, deletion of both ampG and ampP permeases resulted in lowering the levels of disaccharide- muropeptides. There was a minor presence of monosaccharide peptides in the periplasm; namely the 1,6 anhydro MurNAc tri-and tetrapeptides when compared with that seen in whole-cell extracts (Figure 3.6). This concurs with the hypothesis that the major or predominant $N$-acetylglucosaminidase enzyme lies in the cytoplasm, also known as NagZ. However, in agreement with our previous observation of monosaccharides in the periplasm, there may be a periplasmic $\mathrm{N}$-acetylglucosaminidase (Lee, et al., 2016). Indeed, this was recently found to be the case for S. enterica where one of the PG hydrolyzing enzymes of the flagellar operon was found to have this activity (Herlihey, et al., 2014).

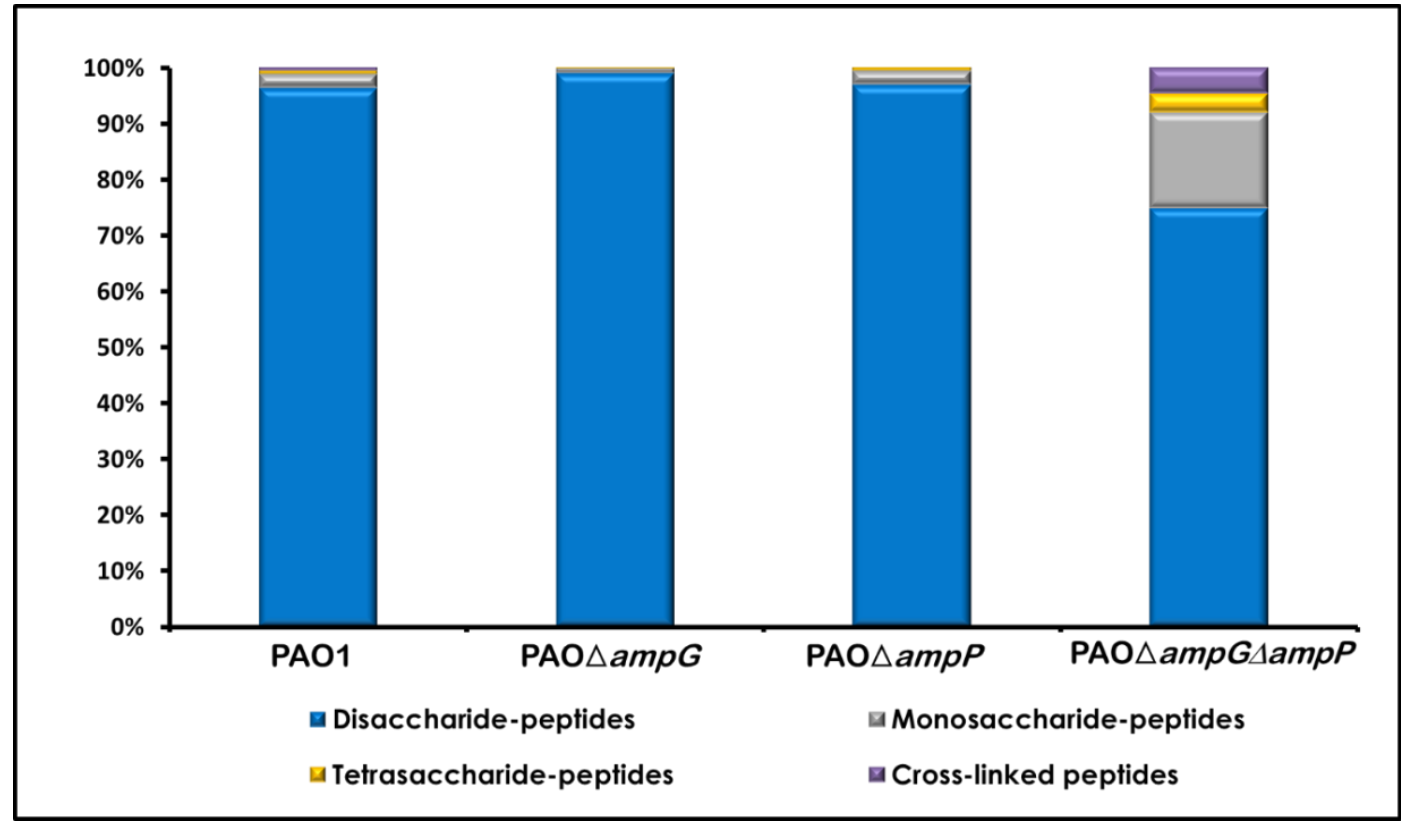


Figure 3.5. Relative levels of muropeptides in periplasm. Muropeptides extracted from the periplasm consisted primarily of disaccharide-peptides across all the strains. Higher levels of monosaccharides were detected in PAO $\triangle a m p G \Delta a m p P$.

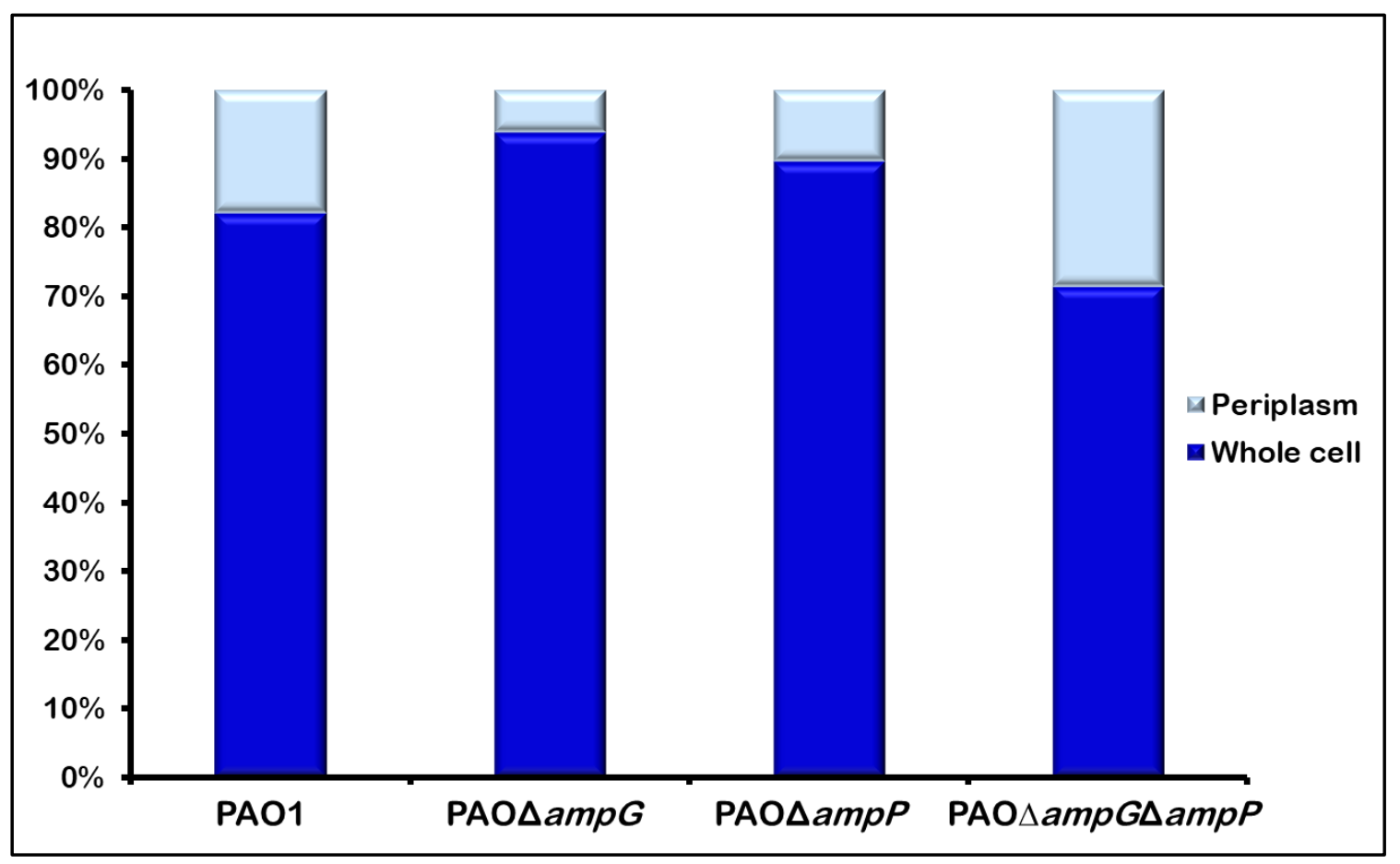

Figure 3.6. Relative levels of monosaccharides in whole-cell and periplasm. 1, 6 anhydro MurNAc peptides tri and tetrapeptide were monosaccharides detected in both whole cell and periplasmic extracts. The levels of monosaccharides in the periplasm were lower when compared to those in the whole-cell extracts.

Similarly, for the larger muropeptides such as the tetra-saccharide peptides and the cross-linked PG fragments, minute quantities were detected in the mutant strains as well as in PAO1. When compared across strains for the whole-cell and the periplasmic extract, levels of the larger peptides were found to be higher (Figure 3.6). This may be due to the sample preparation method as sonication is used to isolate the whole-cell extracts which may result in displacing the larger muropeptides that may otherwise be stuck with the sacculus (Lee, et al., 2016). 


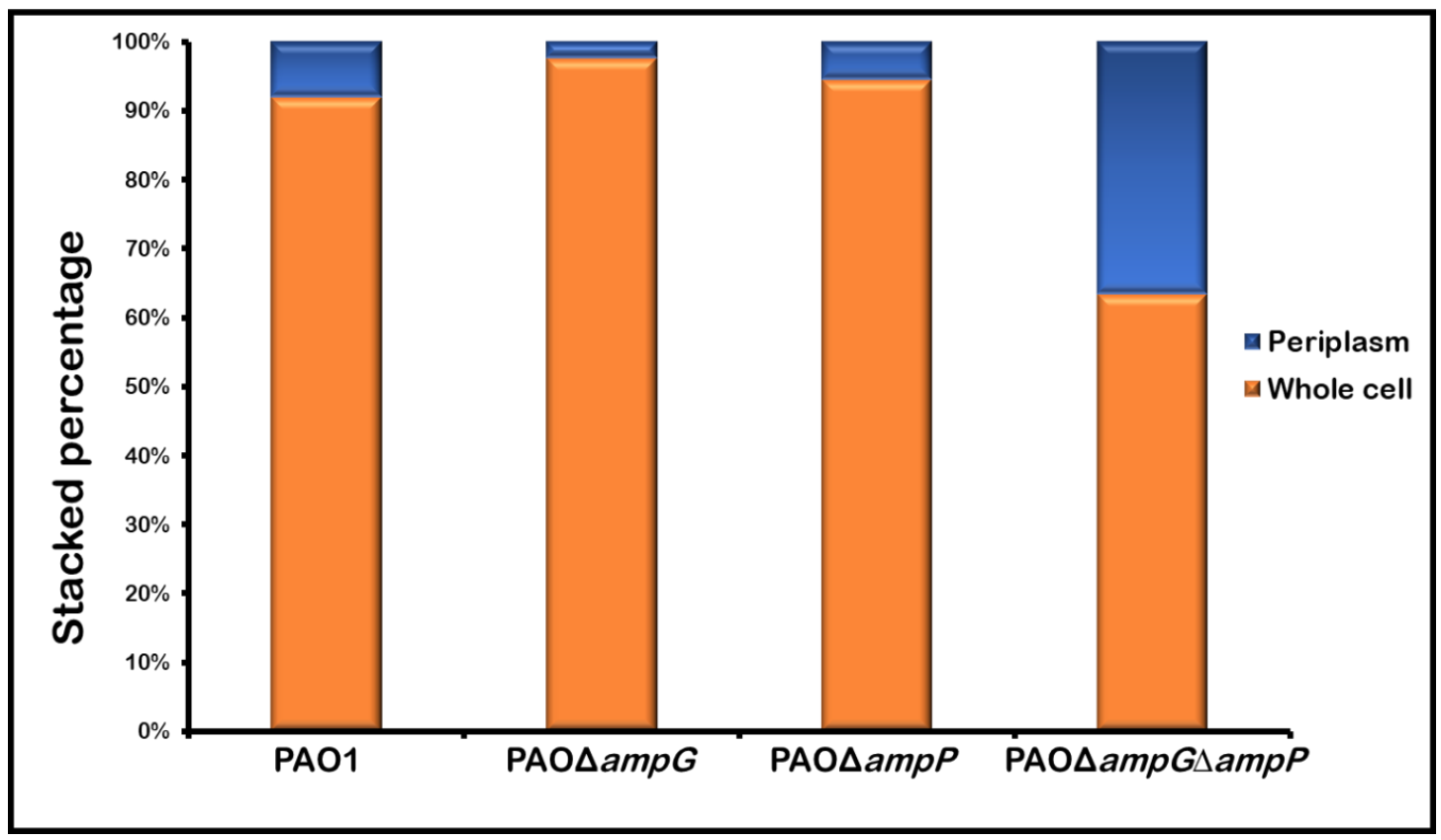

Figure 3.7. Relative levels of larger muropeptides in whole-cell and periplasm. The larger peptides included tetrasaccharides tri-tri peptide and tetra-tetra peptides. The crosslinked peptides included disaccharides- tri-tri peptides and tetra-tetrapeptides. Relatively higher levels were found in the whole cell preparation.

\subsubsection{Decrease of disaccharide in PAO $\triangle a m p P$ periplasm and increase presence of} disaccharide in PAODampG periplasm. As we had the levels of muropeptides in the whole-cells and the levels of muropeptides in the periplasm, by subtraction analyses, the muropeptides in the cytoplasm was inferred. Upon comparing the different groups of muropeptides, it was found that decrease in accumulation of disaccharides was found in ampP deletion in the periplasm and an increase of disaccharides was detected in the ampG deletion suggesting that the permease encoded 
by these genes may play a role in import of these muropeptides into the periplasm and cytoplasm, respectively (Figure 3.8).The levels of monosaccharide and larger muropeptides remain similar in all the strains.

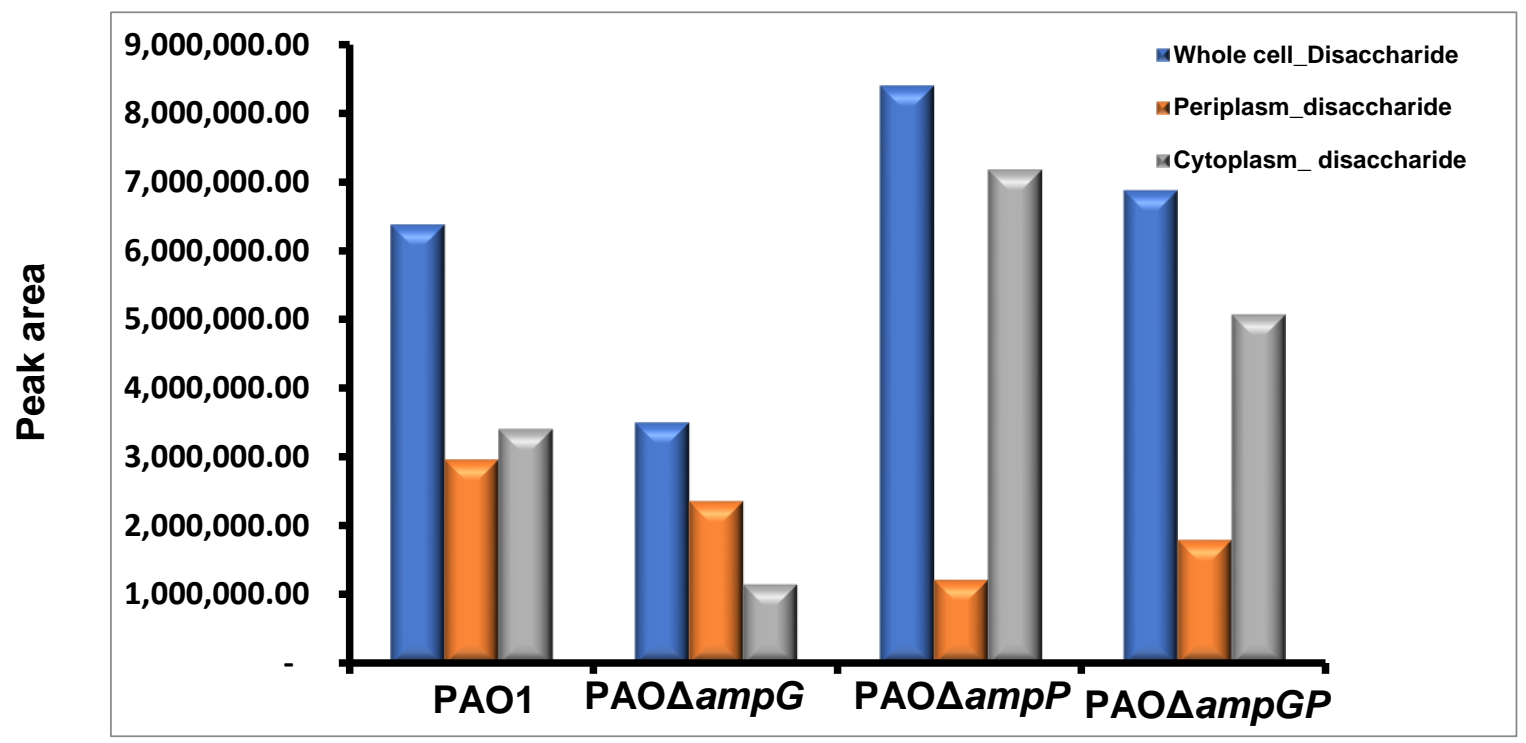

Figure 3. 8. Comparison of disaccharide levels in sub-cellular fractions. The levels of disaccharide muropeptides were calculated in the whole-cell and periplasmic fraction. The levels of muropeptides in the cytoplasm were obtained by subtracting the values of muropeptides in periplasm from whole-cell. It was found that deletion of ampG showed a significant accumulation of muropeptides in the periplasm compared to the wild-type PAO1. The levels of disaccharidemuropeptide in the ampP deletion strain were decreased compared to PAO1. This was also seen upon in the double-deletion strain.

\subsection{Concluding Remarks}

Cell-wall recycling has been researched since the 1960-70s (Chaloupka, et al., 1962, Boothby, et al., 1973, Beck \& Park, 1976). Early studies were performed mostly on $E$. coli or $C$. freundii as model systems. Most of these studies conducted pulse-chase experiments with $\mathrm{H}^{3}$-GIcNAc or $\mathrm{H}^{3}$-DAP which was incorporated in the cell-wall and henceforth recycled and detected in spent media or in cell-fractions (Glauner, et al., 
1988, Dietz, et al., 1997). Muropeptide detection without any radioactivity has been performed only recently (Borisova, et al., 2016, Lee, et al., 2016). Pseudomonas aeruginosa, a portentous opportunistic pathogen exhibits an exceptional capability to resist antibiotic treatment. In this study we explored the link of $\beta$-lactam resistance with the two permeases AmpG and AmpP in cell-wall recycling by comparing the wild-type PAO1 with isogenic single and double-deletion permease mutants. The involvement of the permeases in antibiotic resistance was investigated by E-test and $\beta$-lactamase assay. We also looked at the levels of muropeptides in vivo in $P$. aeruginosa without employing any radioactivity. Studying muropeptides in vivo poses its own challenges. Muropeptides in the bacteria are continuously being turned -over and hence obtaining a snapshot of this dynamic process is technically challenging. One of the main technical difficulties, while quantifying the PG fragments at a certain phase of bacterial growth, is quenching the recycling enzymes namely the amidases, lytic transglycosylases, and the $\mathrm{N}$-acetyglucosaminidases that are usually involved in muropeptide processing. We find that quenching enzyme activity to a large extent can be obtained by boiling the cellextracts without lessening the muropeptide levels.

Previous studies involving non-pathogenic and pathogenic bacteria suggested that ampG plays a role in cell-wall recycling as a permease (Park, 1993). It has also been shown that pathogenic bacteria such as $B$. pertussis and $N$. gonorrhea have an inefficient AmpG and when compared with non-pathogens such as E. coli (Chan \& Dillard, 2016). Both AmpG and AmpP are permeases in $P$. aeruginosa that may play a role in cell-wall recycling. It is shown here that the presence of these permeases is not essential for the growth of the bacteria in a rich medium. Since the elucidation of recycling process and its connection to $\beta$-lactamase already that AmpG is used to import 
the muropeptides into the cytoplasm from the periplasm. Absence or deletion of this permease often results in increase release of muropeptides to extracellular milieu which triggers an inflammatory response in the host (Garcia \& Dillard, 2008). However, this was not seen in $P$. aeruginosa wherein deletion of AmpG resulted in similar total levels of muropeptides as in PAO1. AmpP is unique to the Pseudomonads (Kong, et al., 2010). In $P$. aeruginosa, overall trends of muropeptides were similar across the strains. One of the findings was that our periplasmic preparation yielded less monosaccharides compared to the whole-cell. This is in accordance with the localization of the enzymes in the recycling pathway and indicates that the method of extraction developed here can be used for studying periplasmic PG fragments. We also found increased relative levels of disaccharide in the periplasm of ampG deletion cells and the vice-versa for $a m p P$ and ampG ampP deletion. This confirms the earlier reports of AmpG importing disaccharides and also suggests that AmpP may play a role in transporting disaccharide-muropeptides from the cytoplasm into the periplasm. Although, a permease assay done with $E$. coli spheroplasts using fluorophore- conjugated GlcNAc-MurNAc showed that AmpG was the only muropeptide importer whereas AmpP was unable to import (Perley-Robertson, et al., 2016). However, our speculation is that AmpP may be involved in export of disaccharide muropeptide rather than import.

Significantly diminished MIC for selected $\beta$-lactams and a decrease in $\beta$-lactamase expression was also found in the absence of ampG. This suggests also that the role of AmpG in antibiotic resistance is mediated specifically via the cell-wall recycling pathway and the activation of AmpR. Considering that AmpG is a key player in regulating antibiotic resistance and is not essential for growth, it has been developed as an alternate target for drug development (Collia, et al., 2018). 


\subsection{Acknowledgements}

This work was done in collaboration with Dr. Shahriar Mobashery, Dr. Mijoon Lee and Dr. Dusan Hesek at University of Notre Dame. We are grateful to Dr. Mobashery's lab, particularly Dr. Mijoon Lee for the LC/MS-MS, synthetic muropeptide standards, as well as the discussions and critical analysis of the data towards this research. This work was supported by grant GM61629 from the NIH (to SM) and in-part by NSF IIP-1237818 [PFI-AIR: CRESTI/UCRC-Industry Ecosystem to Pipeline Research] (KM), Florida

International University (FIU) Bridge Funding (KM), FIU Herbert Wertheim College of Medicine Graduate Assistantship (SD). 
Chapter 4

Elucidating the role of PA1085 in Pseudomonas aeruginosa 


\subsection{Abstract}

Infections by the opportunistic human pathogen Pseudomonas aeruginosa are largely intractable due to the various resistance mechanisms demonstrated by this pathogen. One of the primary mechanisms of resistance towards a class of drugs known as $\beta$-lactams is the expression of a hydrolytic enzyme, AmpC. The coordinated activities of the amp genes regulate this enzyme's expression. NagZ is an $N$-acetyl $\beta$-Dglucosaminidase that plays a seminal role in regulating AmpC $\beta$-lactamase expression. LC/MS analyses of muropeptides in PAO1 and the permease mutants eluded that there may be a periplasmic paralog of NagZ. $P$. aeruginosa genome analyses revealed the presence of PA1085 that had $31 \%$ identity with S. enterica FlgJ. We hypothesize that PA1085 is the P. aeruginosa periplasmic glucosaminidase. PA1085 is the fifth gene of a seven-gene operon and a precise in-frame deletion of flgJ (PA1085) was constructed using Gibson cloning. To verify if deleting a gene that is part of the flagellar operon disrupted motility, phenotypic assays such as swimming, swarming and biofilm were performed. For evaluating if flgJ plays a role in regulating $\beta$-lactam resistance the MIC was compared in flgJ deletion strains to the wild-type PAO1 using the Epsilometer test method. An enzyme assay was conducted to determine if assess whether FlgJ possesses $\beta$-N-acetylglucosaminidase activity. We found that flagellar-dependent swimming motility was decreased in the mutant strain, but flagellar and pili-dependent swarming motility were not significantly affected. Our experiments with the deletion strain did not show any differences in the MIC for selected antibiotics. Deletion of PA1085 also did not alter the $\beta$-N-acetylglucosaminidase enzyme activity in whole-cell. 


\subsection{Introduction}

Pseudomonas aeruginosa, an opportunistic, nosocomial pathogen is associated with serious and challenging infections in immunocompromised individuals (Lang, et al., 2004, Sadikot, et al., 2005). In cystic fibrosis patients, with an increase in age, there is a steady decline of lung function and an increase in $P$. aeruginosa colonization (Koch \& Hoiby, 1993, Gisin, et al., 2013). P. aeruginosa infections can at times be resolved with monotherapy but it can also range to highly recalcitrant and deadly episodes. Often for treatment of serious infections, a combination therapy of two or more anti-pseudomonal agents such as aminoglycosides, fluoroquinolones, and $\beta$-lactams is recommended (Flume, et al., 2009, Fothergill, et al., 2012). The underlying factors for the lifethreatening infections are the arsenal of virulence factors and the extraordinary innate and acquired antibiotic resistance mechanisms exhibited by this pathogen (Lister, et al., 2009, Gellatly \& Hancock, 2013). One of the predominant mechanisms of $\beta$-lactam resistance is the expression of chromosomally-encoded AmpC $\beta$-lactamase and its regulation by the global transcriptional factor, AmpR (Lodge, et al., 1990, Lodge, et al., 1993, Kong, et al., 2005). The activity of AmpR as a resistance determinant is further influenced by the products of the cell-wall recycling pathway, known as muropeptides (Wiedemann, et al., 1998, J. F. Fisher, 2015, Lee, et al., 2016, Lee, et al., 2016, Dik, et al., 2017, Dhar, et al., 2018).

Previously, our analyses by liquid chromatography/mass spectrometry identified over 20 muropeptides in $P$. aeruginosa (Lee, et al., 2016). Muropeptides are composed of $N$ acetylglucosamine- $N$-acetylmuramic acid with a peptide side chain (L-Ala- $-\mathrm{D}-\mathrm{D}$-Glu-mesodiaminopimelic acid [DAP]-D-Ala-D-Ala) and are created whenever the bacterial cell divides or elongates and its components are recycled for biogenesis (Glauner, 1988). 
The muropeptides detected by our analysis include disaccharide-peptides such as $N$ acetylglucosamine $\mathrm{N}$-acetylmuramic acid with peptide side chains ranging from zero to five amino acids. Both reduced and non-reduced saccharides were detected (Lee, et al., 2016). Monosaccharides detected include $N$-acetylmuramic acid with peptide side chains. In addition, larger peptides such as cross-linked di- and monosaccharides were also detected (Lee, et al., 2016). In addition to elucidating the structural details of the muropeptides, we were also able to distinguish the GlcNAc pentapeptide and 1.6 anhMurNAc pentapeptides as activating effectors of AmpR (Lee, et al., 2016, Dik, et al., 2017).

Recycling of muropeptides occurs through the combined action of numerous genes dedicated to this pathway (Park \& Uehara, 2008, Johnson, et al., 2013). The groups of enzymes include lytic transglycosylases, amidases, permeases, $N$ acetylglucosaminidases and penicillin-binding proteins (Park, 1993). The muropeptides are translocated into the cytoplasm through permease AmpG (Jacobs, et al., 1997). NagZ $N$-acetylglucosaminidase activity in the cytoplasm leads to the formation of 1,6 anhydroMurNAc peptides which have been identified as the AmpR effectors (Jacobs, et al., 1994, Dietz, et al., 1997, Lee, et al., 2016, Dik, et al., 2017). AmpD amidase cleaves the peptide chain from the muropeptides (Carrasco-Lopez, et al., 2011, Zhang, et al., 2013). Upon $\beta$-lactam induction, loss of $n a g Z$ is associated with a decrease in ampC expression whereas loss of $a m p D$ is associated with an increase in $a m p C$ expression (Jacobs, et al., 1995, Juan, et al., 2005, Juan, et al., 2006, Asgarali, et al., 2009, Lee, et al., 2009). 


\begin{tabular}{|c|c|c|}
\hline \multirow{2}{*}{ Locus Tag } & \multicolumn{2}{|c|}{ P. aeruginosa PA1085 } \\
& \% Identity & \% Similarity \\
\hline S. enterica FlgJ & 31 & 46 \\
P. aeruginosa NagZ & 20.7 & 32.9 \\
\hline
\end{tabular}

Table 4.1. Protein homology between $S$. enterica and $P$. aeruginosa FlgJ. $S$. enterica data was obtained from NCBI. $P$. aeruginosa sequence was obtained from Pseudomonas website (Winsor, et al., 2016). Identity was computed using CLUSTAL W2 "pairwise sequence alignment".

Therefore, NagZ is established as an antibiotic target and small molecule inhibitors of NagZ have been synthesized which impair $\beta$-lactam resistance (Balcewich, et al., 2009, Yamaguchi, et al., 2012, Acebron, et al., 2017). During our analysis of $P$. aeruginosa muropeptides, the NagZ products 1, 6 anhydroMurNAc peptides were detected in the periplasm (Lee, et al., 2016, Lee, et al., 2016). This suggested the existence of a novel periplasmic $\mathrm{N}$-acetylglucosaminidase. A periplasmic $\mathrm{N}$ acetylglucosaminidase FlgJ had been detected in Salmonella sp. (Herlihey, et al., 2014). Bioinformatics analysis of the $P$. aeruginosa genome with $31 \%$ identity to $S$. enterica FlgJ (Table 4.1). Furthermore, sequence alignment of $P$. aeruginosa FlgJ and $S$. enetrica FlgJ shows that the catalytic residue (Glutamate 184) is conserved (Figure 4.1). 


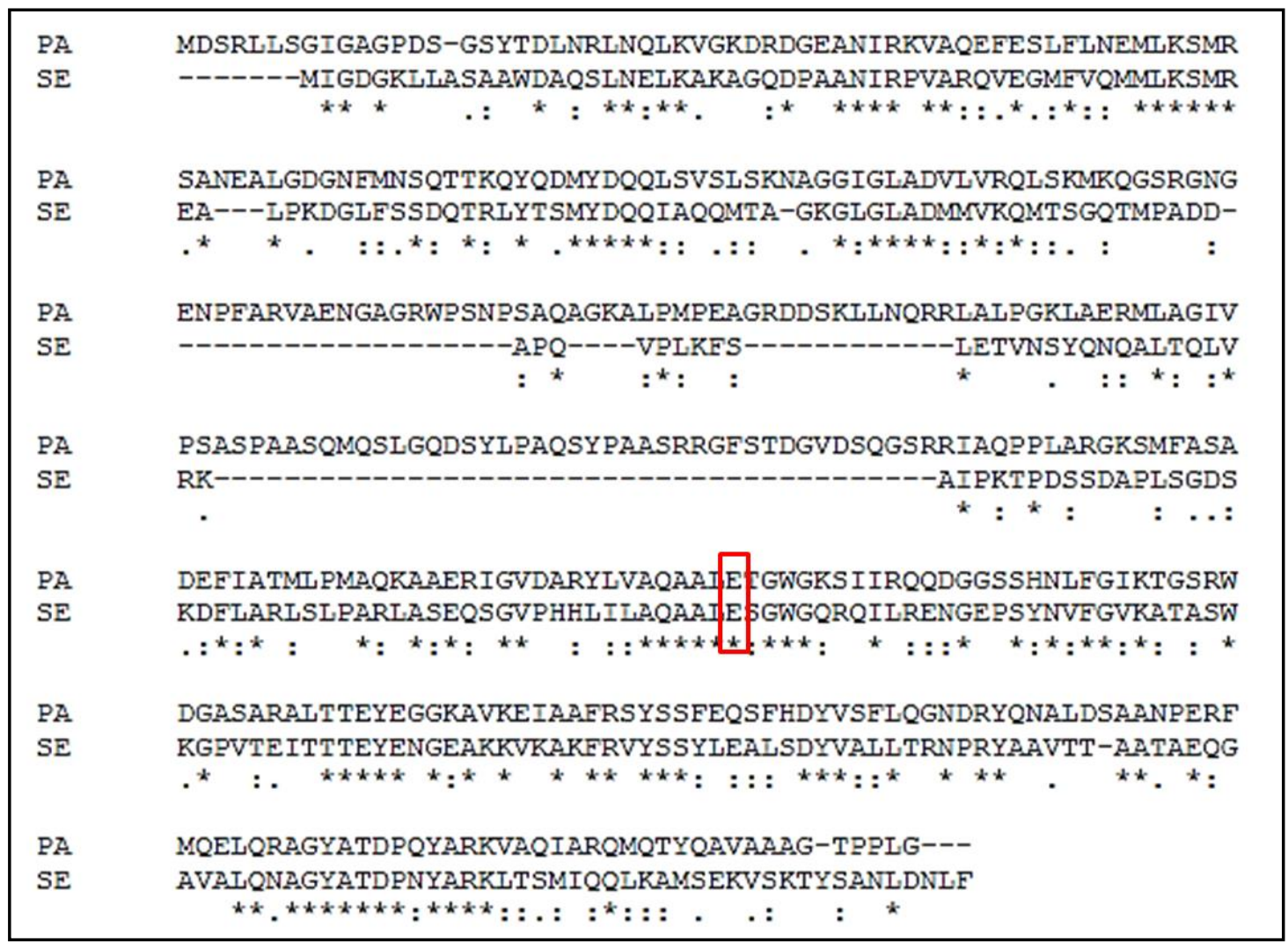

Figure 4. 1. Amino acid sequence alignment of PA1085/FlgJ with S. enterica FlgJ (Lee, et al., 2016). Residues in the red box denote the Glu residues which are predicted as the catalytic acid/base residues in the respective enzymes. Protein alignment was done using CLUSTAL W2 (Winsor, et al., 2016).

The synthesis and assembly of the flagellar system have been best investigated in Salmonella species (Aizawa, 1996, Apel \& Surette, 2008). The C-terminal domain of FlgJ has a peptidoglycan lytic activity whereas the $\mathrm{N}$-terminal domain is required for proper flagellar assembly (Nambu, et al., 1999, Hirano, et al., 2001). FlgJ is part of the the flagellar biosynthesis operon in $P$. aeruginosa (Dasgupta, et al., 2003). The flagellar biosynthesis genes are clustered in three regions on the PAO1 genome (Dasgupta, et al., 2003). Region I comprises of genes that encodes proteins which build the flagellar structure (Dasgupta, et al., 2003). Region II encodes for the accompanying regulatory components whereas Region III encodes for proteins which help in export of the flagella 
and other chemotaxis regulatory factors (Dasgupta, et al., 2003). FlgJ is encoded by the fifth gene of a six gene operon flgF-flgG-flgH-flgl-flgJ-flgK found in Region I. All the other genes in this operon encode structural components of flagella whereas FlgJ is hypothesized to act as a peptidoglycan specific muramidase (Dasgupta, et al., 2003).

FlgJ was found to have $\mathrm{N}$-acetyl $\beta$-D glucosaminidase activity in Salmonella species and it is likely that this enzyme may have a similar function in $P$. aeruginosa (Figure 4.2). The overall goal of this research project is to investigate the role of flgJ in $P$. aeruginosa. Specifically, its role in antibiotic resistance will be ascertained. In addition, the enzyme activity of FlgJ will also be discerned through enzyme assays. Besides its putative role in cell-wall recycling and antibiotic resistance, as this gene is a part of flagellar biosynthesis assembly, its role in motility and motility- associated phenotypes will also be investigated.

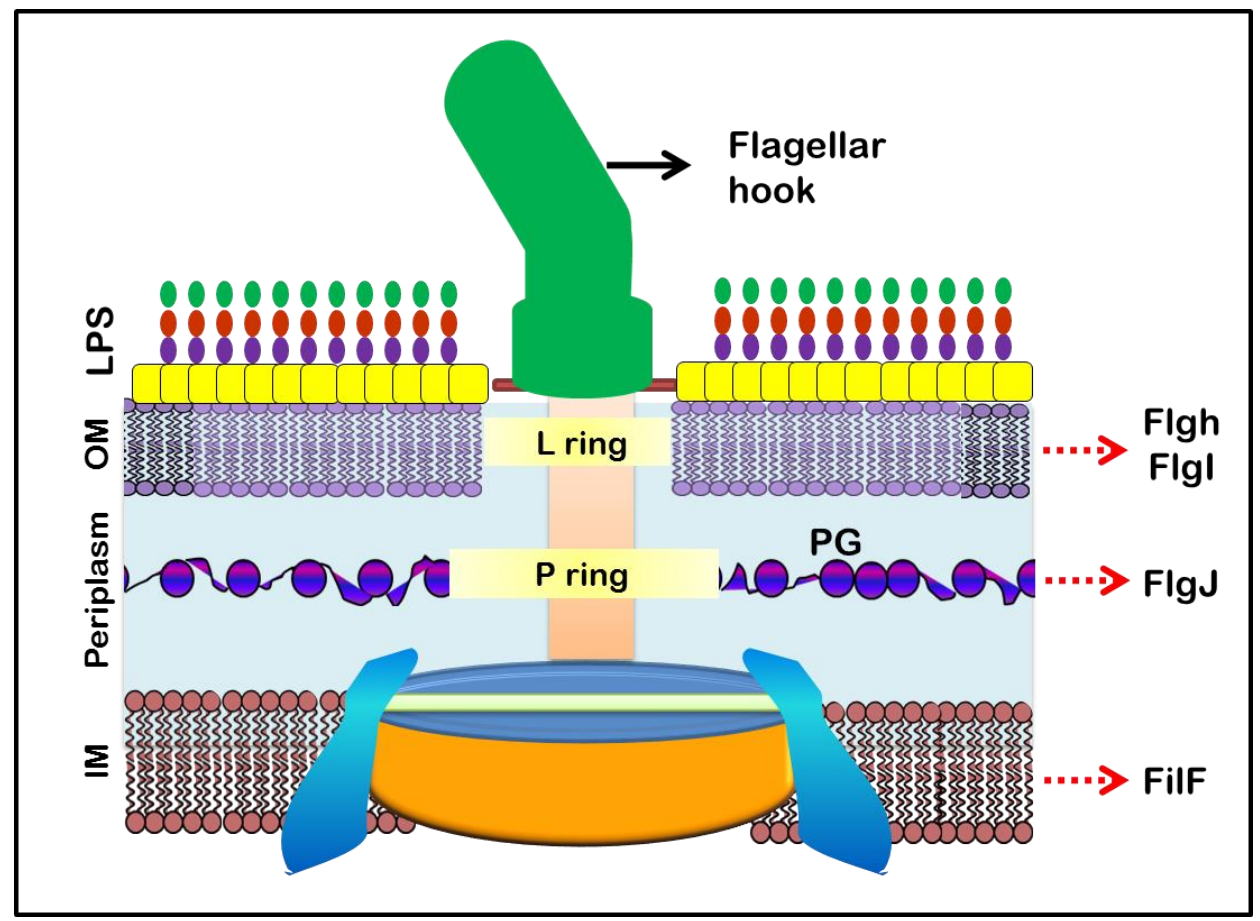


Figure 4. 2. Flagellar assembly. The model for flagellar assembly is best studied in Salmonella sp. The flagellum has to cross through three main barriers, the inner membrane, the peptidoglycan layer and the outer membrane to reach the host/extracellular milieu. This transport process is facilitated by the flagellar transport system (Aizawa, 1996). The transport of the flagellar rod through the inner membrane is achieved by creating gaps by the activity of FliF (Ueno, et al., 1992). FlgJ creates holes in the $P G$ layer allowing its export through the periplasmic space and through the peptidoglycan (PG) layer. PG fragments are produced at this point which may enter the $P G$ recycling pathway and may also act as AmpR effectors. FlgH and Flgl allow the penetration of the flagellar rod through the outer membrane (Vogler, et al., 1991). Land $P$ rings are usually formed at the intersection of the flagellar rod with the outer membrane and PG, respectively (Jones, et al., 1989, Nambu, et al., 1999).

\subsection{Materials and Methods}

\subsubsection{Strains, media and growth conditions. Pseudomonas aeruginosa PAO1} (Stover, et al., 2000) and its derivatives (PAODflgJ) and Escherichia coli DH5a were used in this study. For triparental mating experiments, Pseudomonas Isolation Agar (Difco, NJ, USA) was used along with LB (1:1) for $P$. aeruginosa selection. For plasmid selection and maintenance, antibiotics at the following concentrations were used: Gentamicin (Gm) $15 \mu \mathrm{g} / \mathrm{mL}$ for E. coli, $75 \mu \mathrm{g} / \mathrm{mL}$ for $P$. aeruginosa, Carbenicillin (Cb) at $50 \mu \mathrm{g} / \mathrm{mL}$ for E. coli, $150 \mu \mathrm{g} / \mathrm{mL}$ for $P$. aeruginosa, Chloramphenicol $(\mathrm{Cm})$ and Kanamycin $(\mathrm{Km})$ at $20 \mu \mathrm{g} / \mathrm{mL}$ for E. coli.

Table 4. 2. Strains, plasmids and primers used in this study.

\begin{tabular}{|l|l|l|}
\hline Strains & \multicolumn{1}{|c|}{ Relevant phenotype and genotype } & \multicolumn{1}{c|}{$\begin{array}{c}\text { Reference/ } \\
\text { Source }\end{array}$} \\
\hline Escherichia coli & $\begin{array}{l}\text { F- } \text { Ф80lacZ } \triangle M 15 \Delta(\text { lacZYA argF)U169 deoR } \\
\text { recA1 endA1 hsdR17(rk-mk+) phoA supE44 } \\
\lambda^{-}- \\
\text {thi-1 gyrA96 relA1 }\end{array}$ & $\begin{array}{c}\text { New England } \\
\text { Biolabs }\end{array}$ \\
\hline PH5a & Wild-type & $\begin{array}{c}\text { (Holloway, et } \\
\text { al., 1979) }\end{array}$ \\
\hline PAO1
\end{tabular}




\begin{tabular}{|c|c|c|}
\hline $\mathrm{PAO} \Delta f \operatorname{lgJ}$ & PAO1 containing in-frame deletion of $f / g J$ & This study \\
\hline PAO1(pET 15bvp) & PAO1 containing plasmid pET15BVP & This study \\
\hline PAO $\triangle f l g J(p F l g J)$ & $\begin{array}{l}\text { PAO } \Delta f l g J \text { containing flgJ on plasmid } p \\
\text { ET15BVP }\end{array}$ & This study \\
\hline \multicolumn{3}{|l|}{ Plasmids } \\
\hline pGEMT & $\begin{array}{l}\text { TA cloning vector for PCR products; } \mathrm{Ap}^{R} \text {, } \\
\text { ColE1 } 1 \text { 1 ori lacZa }\end{array}$ & Promega \\
\hline pRK600 & $\begin{array}{l}\mathrm{Cm}^{\mathrm{R}} ; \text { colE1 tra }{ }^{+} \mathrm{RK} 2 \mathrm{mob}^{+} ; \text {Helper plasmid for } \\
\text { conjugation }\end{array}$ & $\begin{array}{c}\text { (Figurski \& } \\
\text { Helinski, 1979) } \\
\end{array}$ \\
\hline pRK2013 & $\begin{array}{l}\mathrm{Km}^{\mathrm{R}} \text {; colE1 tra+ RK2 } \mathrm{mob}^{+} \text {; Helper plasmid for } \\
\text { conjugation }\end{array}$ & $\begin{array}{l}\text { (Finan, et al., } \\
\text { 1986) }\end{array}$ \\
\hline pEXG2 & GmR; pMB1 ori sacB & $\begin{array}{l}\text { (Rietsch, et al., } \\
\text { 2005) }\end{array}$ \\
\hline pET15bVP & $\begin{array}{l}\text { ApR; pBR322 origin, oriV, expression vector, } \\
\text { T7 promoter, His tag coding sequence }\end{array}$ & $\begin{array}{c}\text { (Arora, et al., } \\
\text { 1997) }\end{array}$ \\
\hline pSD194 & $\begin{array}{l}\mathrm{Ap}^{\mathrm{R}} ; \mathrm{A} \sim 1.2-\mathrm{kb} \text { Xhol-BamHl fragment } \\
\text { containing flgJ ORF (PA1085) amplified from } \\
\text { PAO1 } \\
\text { genome using PA1085_fw_Xho1 and } \\
\text { PA1085_Rev_BamH1 primers and cloned } \\
\text { into pGEMT }\end{array}$ & This study \\
\hline pSD197 & $\begin{array}{l}\mathrm{Gm}^{\mathrm{R}} \text {; The flgJ ORF sub-cloned from pSD194 } \\
\text { as an Xhol-BamHl fragment into pET15BVP }\end{array}$ & This study \\
\hline \multicolumn{3}{|l|}{ Primers } \\
\hline Gibson_flgJ_Fw & $\begin{array}{l}\text { 5'- } \\
\text { tgggctagcgaattcgagctAGGAGATATACCATGG } \\
\text { ATTCCCGTTTGCTCAGC } \\
-3 \text { ' }\end{array}$ & This study \\
\hline SD_PA1085_UR & $\begin{array}{l}\text { 5'- } \\
\text { CGGACATGGGTCAGTTCCTCCTTGCTAGC } \\
\text { TAGCTAGGCGTCGTCCTCAAATCACG-3 }\end{array}$ & This study \\
\hline SD_PA1085_DF & $\begin{array}{l}\text { 5'- } \\
\text { CGTGATTTGAGGACGACGCCTAGCTAGCT } \\
\text { AGCAAGGAGGAACTGACCCATGTCCG-3' }\end{array}$ & This study \\
\hline Gibson_flgJ_Rev & $\begin{array}{l}\text { 5'- } \\
\text { ggatccccgggtaccgagctTCAACCCAGAGGCGG } \\
\text { CGT } \\
-3^{\prime}\end{array}$ & This study \\
\hline PA1085_fw_Xho1 & $\begin{array}{l}\text { 5'- } \\
\text { ACACTCGAGAGGAGATATACCATGGATTC } \\
\text { CCGTTTGC-3' }\end{array}$ & This study \\
\hline PA1085_Rev_BamH1 & 5'-GGATCCTCAACCCAGAGGCGGCG-3' & This study \\
\hline
\end{tabular}

\subsubsection{Construction of flgJ deletion mutants by homologous recombination using}

Gibson cloning. A deletion strain of flgJ (PA1085) was constructed using a Gibson 
cloning kit (Gibson, et al., 2009). The DNA sequences upstream (P1) and downstream (P2) of the gene to be deleted were amplified by PCR using High-fidelity polymerase (Pfu DNA Polymerase, Promega, Madison, WI) and primer pairs Gibson_flgJ_Fw, SD_PA1085_UR and SD_PA1085_DF, Gibson_flgJ_Rev (primers listed in table 4.2). The amplicons generated were fused with the EcoR1-linearized plasmid pEXG2 to regenerate the recombinant plasmid (Hoang, et al., 1998). The amplicons were sequenced to ensure fidelity. Henceforth, a similar protocol as used for deletions by yeast-method was followed. The recombinant plasmid was conjugated into $P$. aeruginosa PAO1 by tri-parental mating for homologous recombination with PAO1 genome (Figurski \& Helinski, 1979, Finan, et al., 1986). The colonies that showed integration of the deletion product, as well as the presence of the target gene, also known as single-crossovers, were selected for generating double-crossovers. The double-crossovers were identified by screening for their gentamycin sensitivity and sucrose resistance. The deletion product was verified by PCR amplification and sequencing. Henceforth, this strain is referred to as PAO $\Delta f \lg J$.

4.3.3 Construction of $f l g J$ complementing clones. The flgJ ( 1.2 kb) gene was PCR amplified using primer pairs PA1085_fw_Xho1 and PA1085_Rev_BamH1 (Table 4.2) from $P$. aeruginosa PAO1 genome. The PCR-amplified product was cloned into pGEMT (Promega, Madison, WI). The fidelity of the PCR-amplified product was confirmed by sequencing. The insert carrying flgJ was then moved into a broad-host range pET15bVP plasmid as Xho1-BamH1 fragment, henceforth known as pFlgJ (Arora, et al., 1997). These plasmids were introduced by triparental mating into PAO $\Delta$ flgJ. 
4.3.4 Minimum-inhibitory concentration (MIC). The MIC was determined using the Etest (BioMerieux), which is extensively viewed as a reliable method of susceptibility analyses (Jorgensen, 1993, Amsler, et al., 2010). Briefly, overnight cultures of bacteria were diluted to an O.D. of 0.02-0.2 and spread on a Mueller-Hinton-agar plate. Following this, an E-test containing antibiotics at an increasing concentration along the length of the strip was placed on the plate. The MIC was determined by the zone of clearance on the plate after $16-18 \mathrm{hr}$ incubation at $37{ }^{\circ} \mathrm{C}$.

4.3.5 Motility assays. Swimming assays were done by stabbing $0.3 \%$ LB agar plates with a single colony of PA01, PAO $\Delta f l g J, P A O 1(p E T 15 b V P)$, and PAO $\Delta f l g J(p F l g J)$ bacteria (Henrichsen, 1972). Swarming plates made of $0.5 \%$ LB agar were inoculated with a single colony of the above-mentioned strains and incubated at $30^{\circ} \mathrm{C}$ overnight (Allison \& Hughes, 1991). The swimming and swarming zone were measured after overnight incubation at $30^{\circ} \mathrm{C}$.

4.3.6 Biofilm assay. Biofilm assay was performed in tubes as described previously (Friedman \& Kolter, 2004). Briefly, overnight cultures of PAO1 and mutant strains were grown on LB agar plates were resuspended in LB and diluted to final OD of 0.0025 . $\mathrm{PAO} \Delta$ retS and $\mathrm{PAO} \Delta a \mathrm{lg} D \Delta$ pel$\Delta p s$ l were kept as positive and negative controls, respectively. All the tubes were incubated at $37{ }^{\circ} \mathrm{C}$ for 24 hours. Subsequently, the culture was discarded, and the tubes washed in bins containing water and left at room temperature for ten minutes. This was stained with $0.1 \%$ crystal violet. The dye was removed after 20 minutes and the tubes were washed thoroughly with running tap-water. The attached dye was then solubilized with absolute ethanol and biofilm formation was quantified by recording the absorbance at $590 \mathrm{~nm}$. 
4.3.7 $\mathrm{N}$-acetylglucosaminidase assay. This assay was performed as stated previously (Asgarali, et al., 2009). Briefly, the whole cell and periplasmic fractions from bacteria at late-log phase were extracted. To the sample buffer containing 1xPBS, the substrate 4methylumbelliferyl $\mathrm{N}$-acetyl $\beta$-D-glucosaminide (4-MUGlcNAc) was diluted to a final concentration of $2 \mathrm{mM}$. Extracts containing $20 \mu \mathrm{g}$ of protein was added to a $100 \mu \mathrm{l}$ PBS with substrate. This was incubated at $37^{\circ} \mathrm{C}$ for 4 and $8 \mathrm{hrs}$. At the end of each time-point the reaction was quenched by adding $900 \mu \mathrm{l}$ of $0.1 \mathrm{M}$ glycine- $\mathrm{NaOH}(\mathrm{pH}-10.5)$. The fluorescence was measured by using an excitation wavelength of $360 \mathrm{~nm}$ and monitoring the emission wavelength of $450 \mathrm{~nm}$ on a Synergy H1 Hybrid Reader (Biotek, Winooski, VT). The assay was carried out in triplicates on black 96 well-plates with optical bottom (Thermo Fisher). Controls contained wells with PBS and substrate (2 mM of 4-MUGICNAc), and PBS alone. As a control, PAO $\Delta$ nagZ was used which has a deletion of the gene encoding the cytoplasmic $\mathrm{N}$-acetylglucosaminidase NagZ/PA3005. The results were quantified by measuring the fluorescence against our experimental time-points.

\subsection{Results}

4.4.1 $\mathrm{flgJ}$ is required for swimming motility. Motility is one of the primary virulence factors of $P$. aeruginosa. $P$. aeruginosa shows three main types of motilities which include swimming, swarming and twitching (Henrichsen, 1972). Swimming occurs on a relatively more liquid surface as compared to swarming ( $0.3 \%$ agar vs $0.6 \%$ agar). Swimming is mainly a flagella-regulated movement whereas swarming is regulated partially by flagella and pili (Henrichsen, 1972, Rashid \& Kornberg, 2000). It was found that deletion of flgJ led to a deficiency in swimming motility when compared to the wild- 
type PAO1, whereas swarming motility was unaffected. This may be since swarming movements are the result of the polar flagellum usually found in $P$. aeruginosa, as well as pili and additional alternative motors (Doyle, et al., 2004, Toutain, et al., 2005).

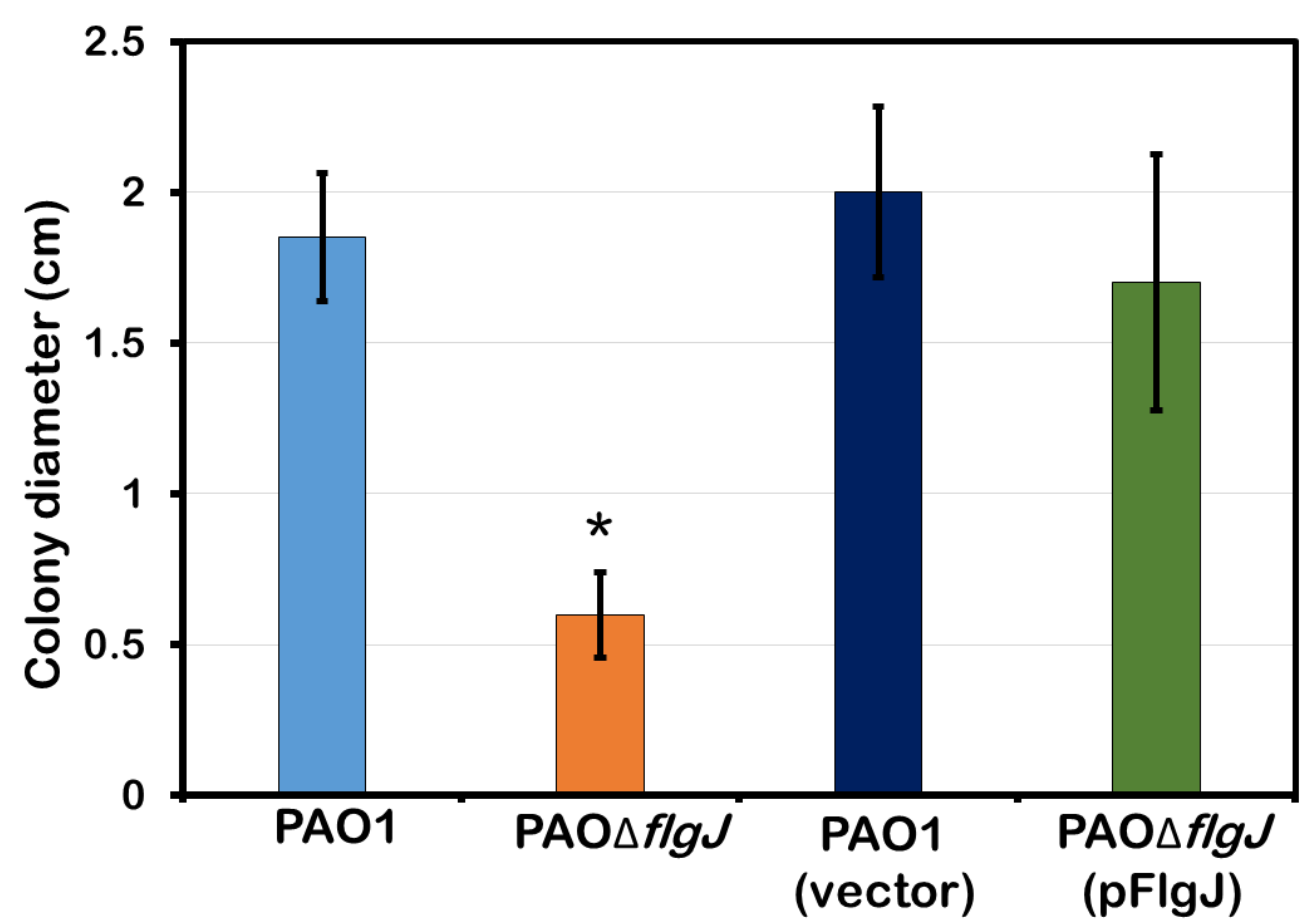

Figure 4. 3. Swimming motility of PAODflgJ vs PAO1. FlgJ was found to be required for regulating swimming motility in PAO1. Values were significant at $p$ $<0.05$ (PAO $\Delta$ flgJ vs PAO1 $p=0.02$, as determined by unpaired student's T-test)

4.4.2 $\mathrm{flg} J$ is required for initiation of biofilm. The formation of biofilms was measured by growing the culture in polystyrene tubes over 24 and 48 hours. Biofilm is an aggregate of polysaccharides, mainly Pel and Psl, extracellular DNA and water channels formed as an extracellular matrix by $P$. aeruginosa (Branda, et al., 2005, Ryder, et al., 2007). An important consequence of this feature is the increased resistance of the bacteria to antibiotics (Stewart, 2002). A biofilm is formed when the bacteria adhere firmly to an abiotic or biotic surface and clinically, is associated with chronic infection 
phenotypes. Quantitation was done by staining the biofilm formed at the air-liquid interphase with $0.1 \%$ crystal violet. PAO $\Delta$ ret $S$ was used as the positive control as retS is involved in inhibition of GacA phosphorylation which promotes biofilm (Goodman, et al., 2009). PAO $\Delta p e l \Delta p s / \Delta a l g D$, a biofilm non-former was used as the negative control for this assay (Balasubramanian, et al., 2012). It was found that in initial stages at 24 hours, where the bacteria attach to the surface, flgJ deletion strains were lagging slightly as compared to the wild-type PAO1, whereas over a time-period of 48 hours, this was negated. In fact, absence of flagella seemed to contribute to higher levels of biofilm formation. This data concurs with previous studies which demonstrate that the flagellum is required only at the initial stages of biofilms.

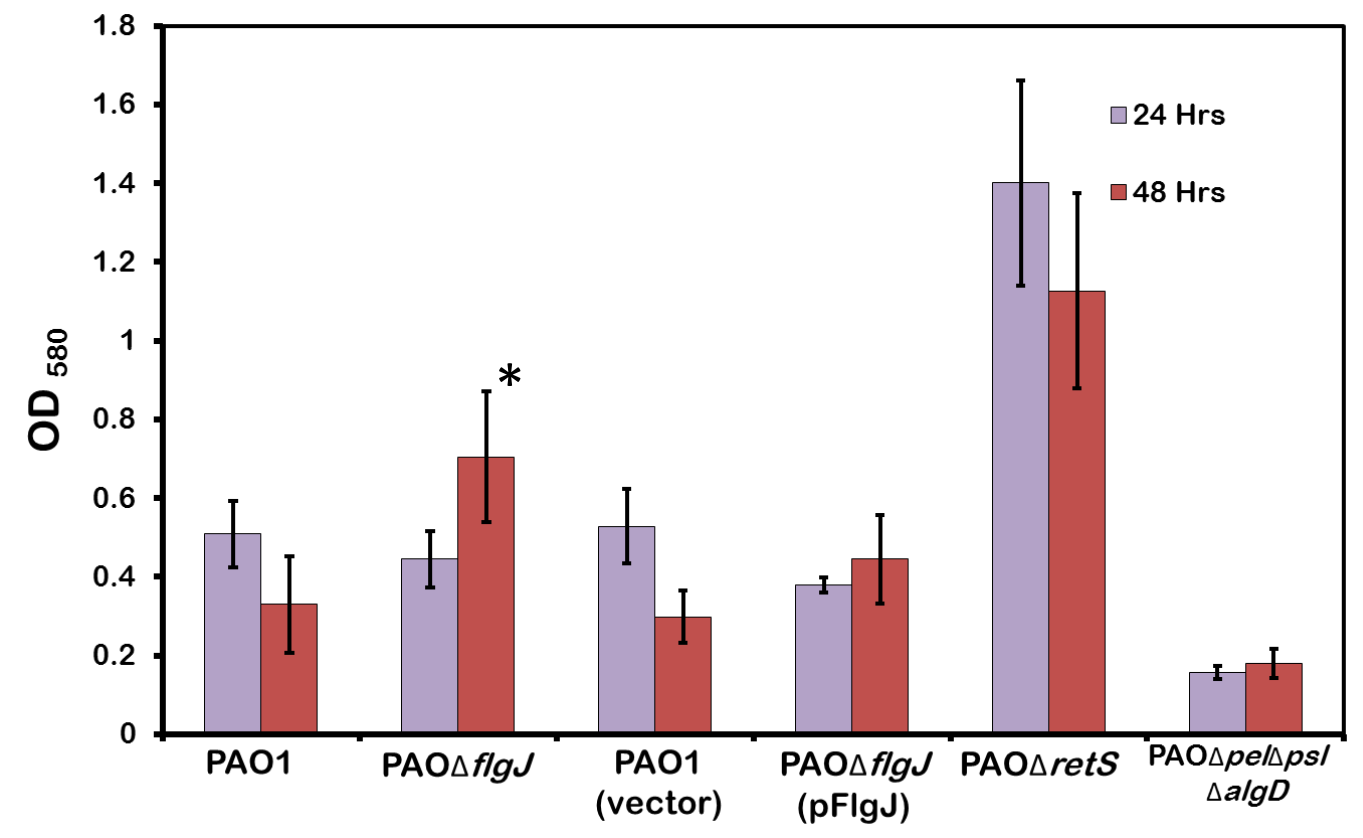

Figure 4. 4. Biofilm formation assay. Biofilms were measured over 24 and 48 hour time-period for all the strains. Differences between the parent PAO1 and deletion was found to be significant at 48 hours ( ${ }^{*} p$ value $=0.03$ as determined by unpaired T-test)

4.4.3 MIC of selected antibiotics is unaltered in absence of flgJ. To investigate the role of flgJ in $P$. aeruginosa, a precise deletion of the gene was constructed in the wild- 
type reference strain, PAO1. The $\beta$-lactam susceptibility of this strain was investigated using the E-test. The antibiotics chosen were based on our hypothesis that FlgJ hydrolyzes the peptidoglycan and generate the monosaccharide muropeptides namely the 1, 6 anhydro peptides which have been shown to act as effectors of AmpR. Hence $\beta$ lactams and other antibiotics which show differential expression in absence of AmpR were investigated (Balasubramanian, et al., 2012). However, no difference was noted in the mutant strain PAOAflgJ in comparison to the parent strain PAO1.

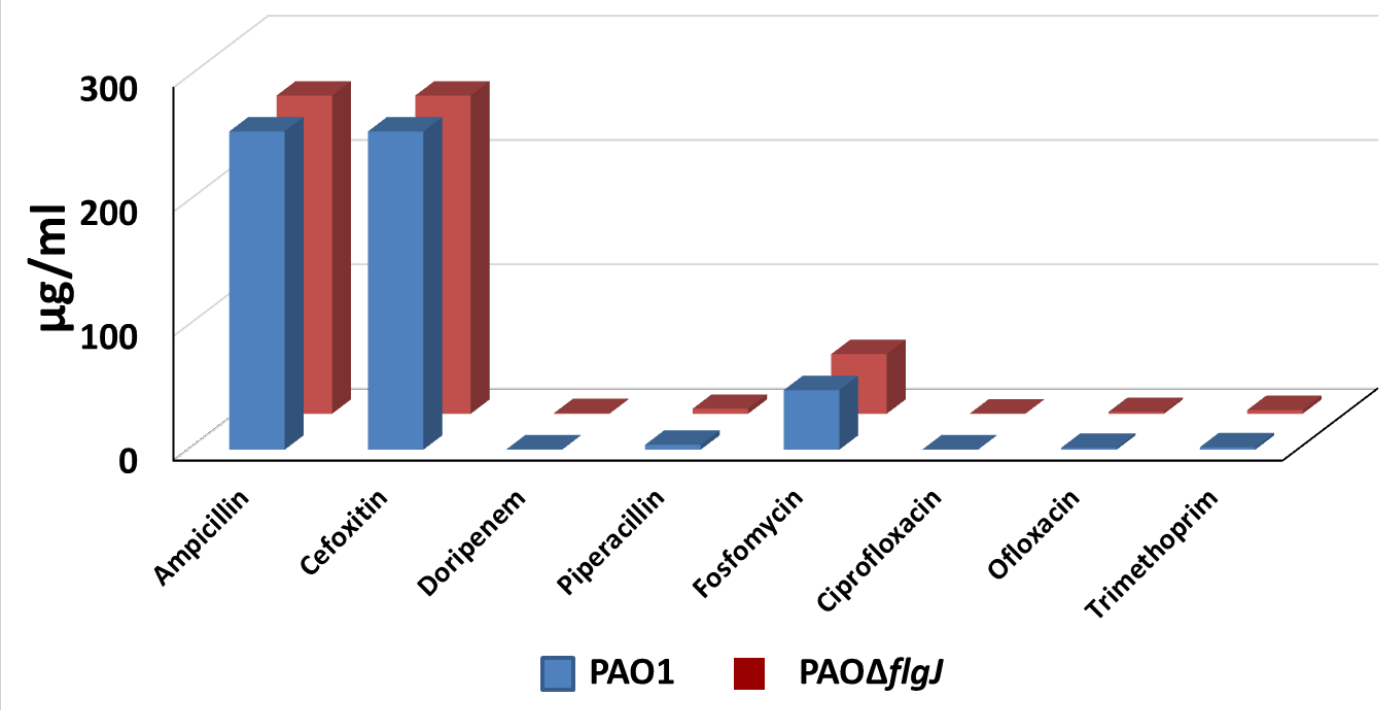

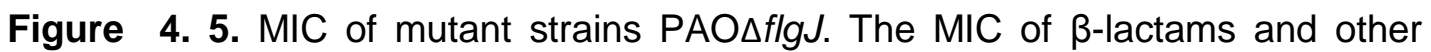
antibiotic that exhibit a differential difference with AmpR deletion was investigated in PAODflgJ and compared to the parent strain PAO1. No significant difference is found between the groups as determined with T-test.

4.4 $\mathrm{N}$-acetylglucosaminidase assay. This assay is based on the conjugation of a fluorogenic substrate, 4- methyl umbelliferyl attached to $N$-acetyl $\beta-D$ glucosaminide (4MU GlcNAc). This assay has been used previously to detect N-acetylglucosaminidase 
activity from lysates of $P$. aeruginosa to determine NagZ expression (Asgarali, et al., 2009). NagZ (PA3005) is currently the only known enzyme in $P$. aeruginosa to possess $N$-acetylglucosaminidase activity. $P$. aeruginosa strains that do not have this gene, hence, have decreased fluorescence in the presence of 4-MUGIcNAc (as was confirmed in this assay). The fluorescence emitted due to the fission of 4-MUGlcNAc from the different bacterial whole-cell extracts were quantified after normalizing with the fluorescence detected from PAOAnagZ. Wells were kept with just the substrate in buffer and the fluorescence recorded from these wells was subtracted from all the test sample wells. When compared with the wild-type PAO1, no significant difference in fluorescence was found in PAO $\mathrm{flgJ}$. As flgJ is believed to be periplasmic, the cleavage of 4MUGIcNAc will be compared between periplasmic fractions of PAO1 and PAO $\Delta$ flgJ.

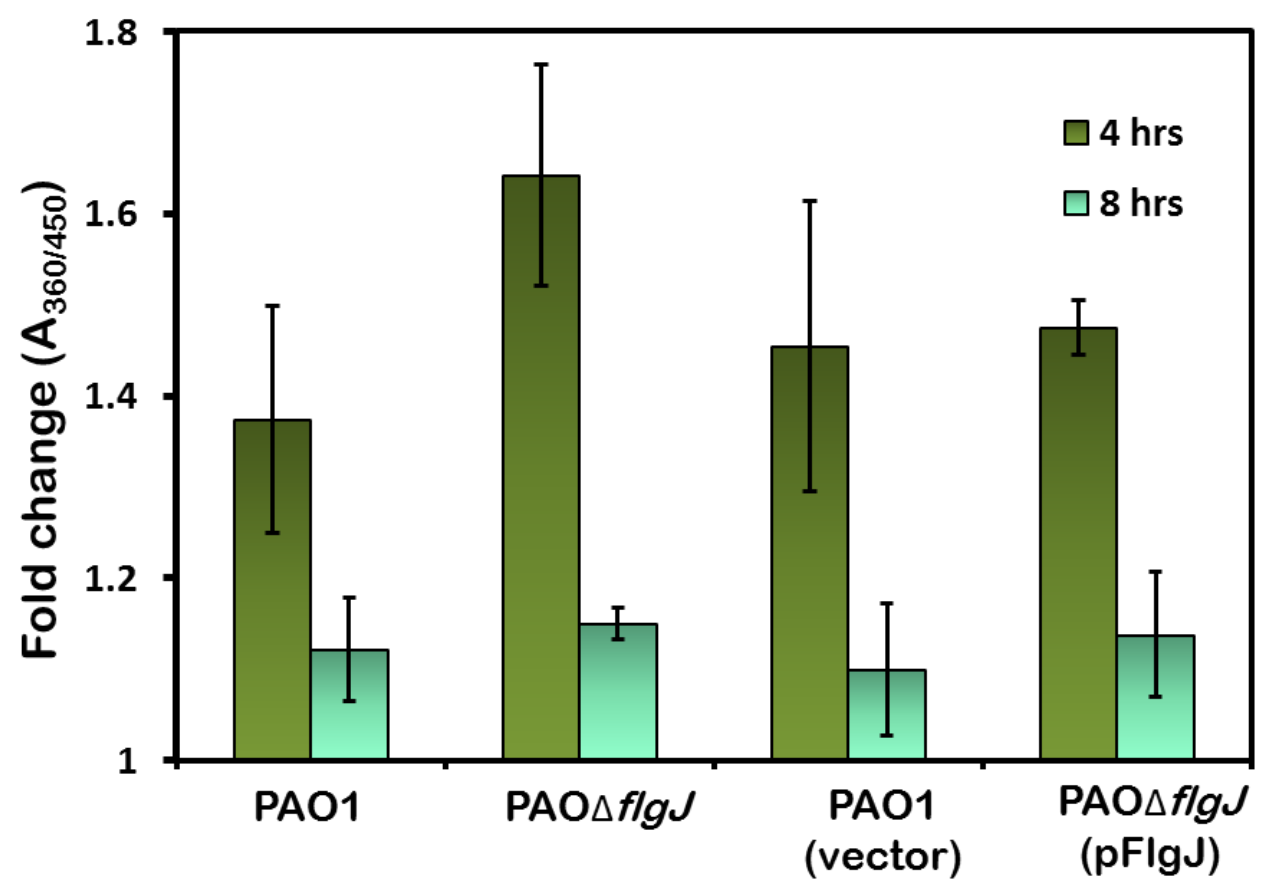


Figure 4. 6. $\mathrm{N}$-acetylglucosaminidase activity in whole-cell extracts. The activity of $\mathrm{N}$-acetylglucosaminidase in whole cell extracts were measured using 4Methylumbelliferyl $\mathrm{N}$-acetyl- $\beta$-D-glucosaminide as a substrate. Fluorescence was measured using an excitation wavelength of $360 \mathrm{~nm}$ and emission at $450 \mathrm{~nm}$. Values were normalized to that of nag $Z$ deletion strain. No significant difference in fluorescence was observed in PAO1 and PAO $\Delta$ flgJ whole-cell lysates ( $p>0.05$, as determined by unpaired students T-test).

\subsection{Discussion}

Cell-wall recycling is closely related to $\beta$-lactam resistance. A particular focus of our lab was to elucidate the profile of muropeptides generated during cell wall recycling in $P$. aeruginosa (Lee, et al., 2016). In our studies, elevated levels of two periplasmic muropeptides GlcNAc anhydroMurNAc- and 1, 6 anhydroMurNAc pentapeptides were detected in the presence of $\beta$-lactams which were later confirmed as the AmpR effector (Dik, et al., 2017). Previous to our in vivo analysis of $P$. aeruginosa muropeptides, 1, 6 anhydroMurNAc were known to be generated exclusively in the cytoplasm following the activity of a $\mathrm{N}$-acetyl $\beta$-glucosaminidase NagZ (PA3005) (Asgarali, et al., 2009). NagZ is established as an antibiotic target and small-molecule inhibitors of NagZ have been synthesized which attenuate $\beta$-lactamase expression and impair $\beta$-lactam resistance (Stubbs, et al., 2007, Yamaguchi, et al., 2012).

During our analysis of muropeptides in $P$. aeruginosa, we detected 1,6 anhydroMurNAc peptides in the periplasm suggesting the existence of a novel periplasmic $\beta-N$-acetylglucosaminidase. A periplasmic $\mathrm{N}$-acetylglucosaminidase FlgJ was identified in $S$. enterica which led us to analyze $P$. aeruginosa FlgJ/PA1085 (Herlihey, et al., 2014). In P. aeruginosa flgJ (PA1085) is a part of the flagellar assembly operon and its role has been associated with flagellar biogenesis. The synthesis of 
flagellar units occurs in the cytoplasm and its assembly and transport is facilitated by the activity of the flagellar transport system (lino, 1974). For the flagella to reach the extracellular milieu, three barriers, namely the inner membrane, PG layer and the outer membrane have to be traversed. It has been presumed that the peptidoglycan layer is broken down by hydrolases that are present in the periplasmic space to facilitate the flagellar insertion (Fein, 1979). The assembly and transport of the flagella have so far not been studied in $P$. aeruginosa. FlgJ in Salmonella was the first account of an $N$ acetylglucosaminidase in the periplasm that breaks the PG allowing the insertion of the flagella and in this process generates monosaccharides (Herlihey, et al., 2014). To understand the role of FlgJ, a precise in-frame deletion was constructed using Gibson cloning. This technique of deletion was adapted as it yields results in a much shorter time than that we previously used involving yeast-system based recombination (Kalva, et al., 2018).

Absence of $\mathrm{flg} J$ resulted in diminished swimming motility and increased biofilm formation which was restored by in trans complementation to the level of wild-type. Motility and chemotaxis are one of the main virulence factors that help in establishing infections in the host. Absence of FlgJ however did not show any difference in the susceptibility profile of selected antibiotics. This would suggest to us that FlgJ is not involved in antibiotic resistance. However, an interesting analysis will be to over-express this protein and observe its effects on the MIC levels. Levels of $N$-acetylglucosaminidase were determined in PAO $\triangle$ flgJ and compared to that of PAO1 using 4-MUGIcNAc as the substrate for $\mathrm{N}$-acetylglucosaminidase. $\mathrm{PAO} \Delta$ nag $Z$ was used as a control and it was found that there was no difference in the levels between the flgJ deletion and the wildtype whole-cell extract. For further analysis the periplasmic extracts will be analyzed for the enzyme activity. 
In Gram-positive organism such as Enterococcus faecalis one of the primary $\mathrm{N}$ acetylglucosaminidases is AtlA (Eckert, et al., 2006). This enzyme comprises of three domains, with a threonine/glutamate rich $\mathrm{N}$-terminal, a catalytic central domain and LysM domains at the C-terminal, each of which is required for the maximal hydrolytic activity of the peptidoglycan (Eckert, et al., 2006). Upon comparisons of the protein domains, we find that PA1085 has a similar conserved LysM domain. The LysM domain is indicative of proteins that are capable of binding with the $P G$ and is hence important for eliciting the full enzymatic activity of the protein (Buist, et al., 2008). The conservation of this sequence in FlgJ further reinforces our hypothesis that this enzyme may have an $\mathrm{N}$-acetylglucosaminidase activity. Domain analysis of PA1085 using the Carbohydrate active enzymes (CAZy) database also reveals that it belongs to the glycoside hydrolase family of proteins GH73 (Cantarel, et al., 2009). Proteins belonging to this family are known to cleave the linkage between N-GlcNAc and MurNAc (Hashimoto, et al., 2009).

Besides FlgJ, genome analysis of $P$. aeruginosa revealed a second periplasmic glucosidase BgIX (PA1726) which is yet to be characterized. Upon multiple sequence alignment of NagZ, FlgJ and BgIX, it was found that one of the active site residues of NagZ, D244, are conserved on both FlgJ and BglX. This suggests the possibility of a different periplasmic hydrolase that may generate the periplasmic monosaccharides. Our research has explored the role of AmpP/PA4218 as an exporter of disaccharidepeptides. Alternatively, there may be another transporter which exports the cytoplasmic monosaccharides into the periplasm. 


\subsection{Acknowledgements}

We would like to that Dr. Brian Mark (University of Manitoba) for providing us with the nagZ strains. This study was funded in part by NIH/NIGMS R25 GM061347 to Supurna Dhar (SD) and Florida International University Dissertation Year Fellowship (SD). 
Chapter 5

Small-Molecule Library Screening For Pseudomonas aeruginosa AmpR Inhibitors 


\subsection{Abstract}

Pseudomonas aeruginosa has been classified by the World Health Organization as a pathogen of "critical" importance. It causes serious acute and chronic infections often resulting in life-threatening episodes. Previous research in our lab has identified players in the amp system which plays a role in antibiotic resistance and virulence. AmpR has an extensive regulon in $P$. aeruginosa and is intricately linked to antibiotic resistance, recycling of the cell-wall and expression of virulence factors required for infection. Although AmpR has been identified in many pathogens, its role in $P$. aeruginosa appears to be more elaborate. In $P$. aeruginosa, AmpR has been shown to localize in the membrane and exist as a dimer in the active form which is further explained in this research through structural modelling. This research also explores AmpR as a novel antibiotic target. A phenotypic screening assay has been developed fusing promoters regulated by AmpR, $\mathrm{P}_{a m p c}$, to a lux operon cassette. This reporter was used to screen scaffold-based combinatorial library for AmpR inhibitors. Positive hits identified from this study will be further counter-screened using reporters with alternate AmpR regulated promoters namely, $\mathrm{P}_{\text {phzA1 }}$ and $\mathrm{P}_{\text {mexE. }}$. Novel compounds that are identified in this study can also be used for other pathogen with a homologous AmpR-AmpC system such as Yesrsinia enterocolitica, Burkholderia cenocepacia, and Strenotrophomonas maltophila.

\section{2. Introduction}

Pseudomonas aeruginosa is known for causing recalcitrant infections in mainly immunocompromised patients and at least $10 \%$ of all nosocomial infections in the US are due to this pathogen (Bodey, et al., 1983, Gaynes \& Edwards, 2005). This 
opportunistic bacterium can cause a wide-range including both chronic and acute infections in humans (Furukawa, et al., 2006). Some of the chronic infections are respiratory tract and lung-associated resulting in chronic pneumonia, pan bronchiolitis, and chronic obstructive pulmonary disorder (Rose, 1968, K. Yanagihara 2001, Boyer, et al., 2005, Martinez-Solano, et al., 2008). One of the most archetypal chronic infections linked with this pathogen is found in cystic fibrosis (CF) patients. CF lungs present with mixed-infections, colonized by $P$. aeruginosa, Staphylococcus aureus, Haemophilus influenzae, Methicillin-resistant S. aureus (Marks, 1990, Rayner, et al., 1990, Koch \& Hoiby, 1993, Tummler, et al., 1997, Miall, et al., 2001, Marshall, et al., 2016). Owing to its incredible multi-factorial pathogenesis and propensity for antibiotic resistance, with an increase in the patient's age, $P$. aeruginosa reigns supreme amongst the other lungassociated infections (West, et al., 2002). These recurrent pulmonary infections result in increased inflammatory responses and lung deterioration, ultimately causing high morbidity and mortality (Yum, et al., 2014, Langan, et al., 2015). Besides chronic, $P$. aeruginosa also possess the virulence arsenal for acute infections (Yahr \& Greenberg, 2004). Notable ones include acute pneumonia, particularly in patients who are hospitalized or are unable to mount a sufficient immune response against $P$. aeruginosa (Pereira, et al., 2014). Other significant ones include acute keratitis occurring in up to $40 \%$ of cases found in US. Besides these, sepsis due to bacteremia and woundinfections due to burns or post-surgery and urinary tract infections are also observed (Wu, et al., 1999, Estahbanati, et al., 2002, Kang, et al., 2003, Secher, et al., 2005, Shigemura, et al., 2006, Migiyama, et al., 2016).

Underlying these two infection phenotypes are distinct groups of virulence factor and their regulators (Furukawa, et al., 2006, Gellatly \& Hancock, 2013). The prominent ones that contribute to chronic infection include biofilm and alginate, often found in a CF lung 
(Pedersen, et al., 1990, Pedersen, 1992, Winstanley, et al., 2016). Acute infection is due to release of exo- and endo-toxins, proteases and involves largely the quorum sensing (QS) and type 3 secretion systems (T3SS) (Smith \& Iglewski, 2003, Hauser, 2009). The decision of an acute or chronic infection in the host is regulated by the environmental signals which determine the gene expression levels of various virulence determinants. Often, the inability to cure an acute infection results in genetic adaptive changes in the organism resulting in a chronic infection (Furukawa, et al., 2006, Balasubramanian, et al., 2013, Winstanley, et al., 2016). Thus, preventing the establishment of an acute infection is of fundamental importance.

Previous studies in our lab have shown that a transcriptional factor involved in regulating acute infection phenotypes is AmpR (PA4109) (Balasubramanian, et al., 2015). AmpR has been the focus of research for long as it was recognized that it is a pivotal point for regulating $\beta$-lactamase resistance (Normark \& Lindberg, 1985, Wiedemann, et al., 1998). In addition to its role as a cynosure in $\beta$-lactam resistance, in P. aeruginosa it also acts as a "global" regulator (Balasubramanian, et al., 2012). The extent of its regulation is limited to not just $\beta$-lactams, but towards many non- $\beta$-lactam antibiotics as well as acute infections- related phenotypes. One of the major areas where AmpR influence is seen is toward the quorum sensing (QS) regulated phenotypes, including production of LasA, LasB proteases, phenazine pigments as well as in biofilm formation (Balasubramanian, et al., 2012). It has been found that AmpR regulates three of the four QS in P. aeruginosa; Las, Rhl, and PQS (Balasubramanian, et al., 2014). These systems together improvise a majority of virulence factors affecting acute infections. AmpR deletion also favors diminished virulence of $P$. aeruginosa in animal models of toxicity (Balasubramanian, et al., 2012). In addition to virulence factors, AmpR is also required for essential metabolic processes that promote survival in the 
host, such as iron uptake and response to oxidative-stress (Balasubramanian, et al., 2012, Balasubramanian, et al., 2014).

Besides regulating multiple virulence and other metabolic processes, $P$. aeruginosa AmpR along with other players of cell-wall recycling also plays a central role in regulating $\beta$-lactam resistance (Kong, et al., 2005). Furthermore, its influence is not limited to just $\beta$-lactams but to other antibiotics such as fluoroquinolones and aminoglycosides (Balasubramanian, et al., 2012). Previous research in our lab has also shown that AmpR creates cross-resistance between antibiotics in the presence of subinhibitory concentrations of $\beta$-lactam antibiotics (Kumari, et al., 2014).

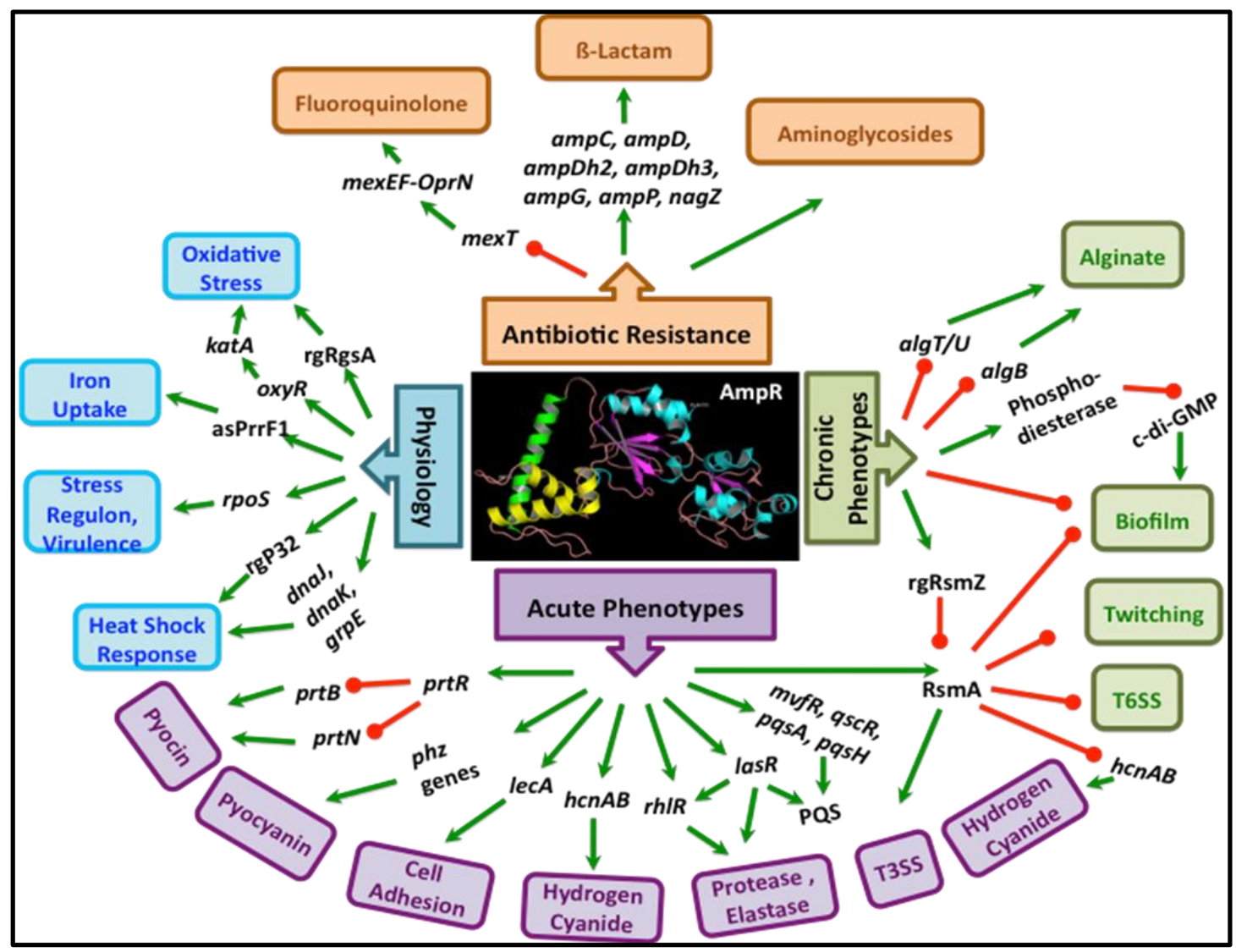


Figure 5. 1. AmpR regulon. AmpR which was previously known to be connected to $\beta$-lactam resistance was shown to have an extensive regulon in $P$. aeruginosa.

Reprinted with permission from Oxford Publishing.

The increasing evidence that AmpR plays a massive role in the pathogenicity of $P$. aeruginosa supports it as a candidate for a novel antibacterial target. The benefits of inhibiting AmpR are multi-fold. The rising antibiotic resistance demands that we look into identifying not just novel small-molecules that can be used as drugs but also develop novel antibacterial targets. The targets that depart from showing lethality towards the bacteria are preferred as that would not evoke rapid evolutionary protective responses from the bacteria (Kaczor, et al., 2017). Rather, any target that deprives the organism of its virulence arsenal and promotes a synergistic effect with other antibiotic targets is preferred (Lynch \& Wiener-Kronish, 2008, Silver, 2011). In addition, the AmpR-AmpC system is found in many pathogens such as Yersinia enterocolitica, Strenotrophomonas maltophila, Burkholderia cenocepacia (Seoane, et al., 1992, Proenca, et al., 1993, Okazaki \& Avison, 2008). Furthermore, this protein does not have its homolog in humans hence makes it unique to bacteria.

This part of my dissertation project deals with further exploring AmpR and its relevance in $P$. aeruginosa. Firstly, the localization of AmpR in membrane is reiterated again, this time using both sucrose-gradient studies. In addition, we also describe a reporter screen constructed for evaluation of small-molecule inhibitors of AmpR. Furthermore, it has been shown that our reporter system can be used to detect for QS inhibitors. 


\subsection{Materials and Methods}

5.3.1. Strains, media and growth conditions. P. aeruginosa PAO1 (Stover, et al., 2000) and its derivatives (PAO $\triangle a m p R)$ and Escherichia coli DH5a were used in this study. For triparental mating experiments, Pseudomonas Isolation Agar (Difco, NJ, USA) was used along with LB for $P$. aeruginosa selection. For plasmid selection and maintenance, antibiotics at the following concentrations were used: Tetracycline (Tet) 20 $\mu \mathrm{g} / \mathrm{mL}$ for $E$. coli, $100 \mu \mathrm{g} / \mathrm{mL}$ for $P$. aeruginosa, Carbenicillin (Cb) at $50 \mu \mathrm{g} / \mathrm{mL}$ for $E$. coli, Chloramphenicol $(\mathrm{Cm})$ and Kanamycin $(\mathrm{Km})$ at $20 \mu \mathrm{g} / \mathrm{mL}$ for $E$. coli and at $50 \mu \mathrm{g} / \mathrm{mL}$ for P. aeruginosa.

5.3.2. Membrane fractionation. Membrane fractions were isolated using previously established protocols. PAO $\triangle a m p R$ and PAO $\triangle a m p R$ (pAmpR-His6) cells were streaked on LB-agar. A single colony was inoculated next day in LB with and without antibiotics and grown until an $\mathrm{OD}_{600}$ of 0.2 . PAO $\triangle a m p R$ (pAmpR-His6) was induced with $1 \mathrm{Mm}$ IPTG and incubated for $6 \mathrm{~h}$ with shaking before harvesting. Cells were recovered by centrifugation at $5,000 \times \mathrm{g}$ for $15 \mathrm{~min}$ at $4^{\circ} \mathrm{C}$ and washed with equal volume of $20 \mathrm{mM}$ of Tris- $\mathrm{Cl}$. The culture was then resuspended in lysis buffer containing $20 \mathrm{mM}$ Tris-cl, $\mathrm{pH} 8$, $0.1 \mathrm{M} \mathrm{NaCl}, 1 \mathrm{mM}$ EDTA, $100 \mu \mathrm{g}$ of DNase I, 1mg/ml Lysozyme and $1 \mathrm{X}$ Roche complete protease inhibitor cocktail. Following disruption of the cells on ice with sonication (15 cycles of 10-s pulse on and 30-s pulse off; amplitude, 40\%), the cell lysate was centrifuged at $4,000 \times \mathrm{g}$ for $10 \mathrm{~min}$ at $4^{\circ} \mathrm{C}$ to remove unbroken cells. The supernatant was ultra-centrifuged at 36,000 rpm (Beckman Coulter Ti45 rotor) for $3 \mathrm{~h}$ at $4^{\circ} \mathrm{C}$, and the pellets containing membrane were resuspended in membrane buffer containing $25 \%$ sucrose, $20 \mathrm{mM}$ Tris, $\mathrm{pH} 7.4$, and1X Roche complete protease inhibitor cocktail. The membrane fractions were aliquoted and stored at $-80^{\circ} \mathrm{C}$. All Western blots were 
developed according to previous protocols. Briefly, equal amounts of proteins were loaded on a $12 \%$ SDS-PAGE gel and run at 100 Volt for $1 \mathrm{~h}$. The proteins were then transferred to a polyvinylidene difluoride (PVDF) membrane (Bio-Rad) and blocked with $3 \%$ BSA in Tris-buffered saline (TBS) at $4^{\circ} \mathrm{C}$ overnight or at room temperature for $1 \mathrm{~h}$. The membrane was washed after blocking with TBST three times for ten minutes each and probed with mouse-anti-His antibody (Biolegend, 1: 3,000) for $2 \mathrm{~h}$ at room temperature or at $4{ }^{\circ} \mathrm{C}$ overnight. Membranes were subsequently washed with TBST, incubated with anti-mouse IgG horseradish peroxidase-conjugated antibody $(1: 5,000)$ (Bio-Rad) for $1.5 \mathrm{~h}$. The membranes were washed with TBST and developed using SuperSignal West Pico Chemiluminescent Substrate (Pierce). The membrane fraction was confirmed using Lactate dehydrogenase enzyme activity assay.

5.3.3. Modeling of AmpR. The monomer structure of AmpR effector binding domain from Citrobacter freundii was obtained from protein data bank (pdb ID 3KOS.pdb). The python script MakeMultimer.py was used to construct the dimer structure from BIOMT transformation matrices contained in the pdb file. The visual molecular dynamics (VMD) software was used to visualize and mutate structure (Humphrey, et al., 1996).

5.3.4. Reporter construction for luciferase assays. Reporters were constructed with fusion of AmpR regulated promoters upstream of the lux operon. Specifically, $\mathrm{P}_{a m p c}$ and $\mathrm{P}_{\text {phzA }}$, which are respectively regulated by $A m p R$, with and without induction, were selected (Balasubramanian, et al., 2012). $P_{a m p c}(\sim 100 \mathrm{bp})$ was amplified from $P$. aeruginosa PAO1 genome using primers EL04_ampC_forward_Xho and EL 05_ampC_reverse_BamH1 and cloned into pGEMT vector (PROMEGA). This was 
ligated into kanamycin resistant pMS402 plasmid (11.8Kb) upstream of a promoterless luxCDABE operon (Olsen, et al., 1982, Duan, et al., 2003). The operon encodes for all the synthase as well as the substrate for luciferase. This plasmid pMS402 $\left(P_{a m p c}\right)$ is henceforth known as pHK478. This plasmid was further digested with Pac1 restriction enzymes to yield an $8 \mathrm{~Kb}$ product that was ligated with Pac 1 digested pCDS101 plasmid containing the CTX backbone including sequences required for integration of plasmid into the Pseudomonas genome. This ligation product is electroporated in to Lucigen Electro-competent cells and plated on tetracycline and kanamycin. Resulting clones were analyzed by PCR and sequenced. Colonies containing the resulting plasmid with $\mathrm{P}_{\text {ampc }}$ is henceforth referred to as HK502. HK502 is then mated into P. aeruginosa PAO1 to yield PAOattB::PampC-lux, henceforth known as HK504. ampC is regulated by AmpR specifically in the presence of an inducer such as imipenem. A second reporter strain is constructed with PphzA which is regulated by $A m p R$ without any inducer. Plasmid pCDS101 contained the PphzA with luxCDABE operon and was introduced into P. aeruginosa $\mathrm{PAO} 1$ and $\mathrm{PAO} \triangle a m p R$ by triparental mating. The final clones are PAOattB::PphzA-lux and PAO $A a m p R a t t B:: P p h z A-l u x$ and are known as SD98 and SD94, respectively. The strains were validated by PCR amplification, sequencing and by phenotypic assay for luciferase activity.

Table 5. 1. Strains, plasmids and primers used in this study.

\begin{tabular}{|l|l|l|}
\hline Strains & \multicolumn{1}{|c|}{ Relevant phenotype and genotype } & $\begin{array}{c}\text { Reference/ } \\
\text { Source }\end{array}$ \\
\hline Escherichia coli & $\begin{array}{l}\text { F- } \Phi 80 \text { lacZAM15 } \triangle(\text { lacZYA argF)U169 deoR } \\
\text { recA1 endA1 hsdR17(rk-mk+) phoA supE44 } \lambda^{-}-\end{array}$ & $\begin{array}{l}\text { New England } \\
\text { thi-1 gyrA96 relA1 }\end{array}$ \\
\hline DH5a & Wild-type & (Holloway, et \\
\hline Pseudomonas aeruginosa & WAO1
\end{tabular}




\begin{tabular}{|c|c|c|}
\hline & & al., 1979) \\
\hline PAO $\triangle a m p R$ & PAO1 containing in-frame deletion of $a m p R$ & \\
\hline $\mathrm{PAO} \Delta a m p R(\mathrm{pAmpR})$ & $\begin{array}{l}\text { PAO } \triangle a m p R \text { containing ampR on plasmid } \\
\text { pMMB67-EH }\end{array}$ & \\
\hline PAOattB::PampC-lux & $\begin{array}{l}\text { PAO1containing } P_{\text {ampc }} \text { upstream of luxCDABE } \\
\text { operon }\end{array}$ & This study \\
\hline PAO1attB::PphzA-lux & $\begin{array}{l}\text { PAO1containing PphzA upstream of luxCDABE } \\
\text { operon }\end{array}$ & This study \\
\hline $\begin{array}{l}\text { PAOAampRattB::Pphz } \\
\text { A-lux }\end{array}$ & $\begin{array}{l}\text { PAO } \triangle a m p R \text { containing PphzA upstream of } \\
\text { luxCDABE operon }\end{array}$ & This study \\
\hline \multicolumn{3}{|l|}{ Plasmids } \\
\hline pGEMT & $\begin{array}{l}\text { TA cloning vector for PCR products; } \mathrm{Ap}^{\mathrm{R}} \text {, } \\
\text { ColE1 f1 ori lacZ } \alpha\end{array}$ & Promega \\
\hline pRK600 & $\begin{array}{l}\mathrm{Cm}^{\mathrm{R}} ; \text { colE1 tra }{ }^{+} \mathrm{RK} 2 \mathrm{mob}^{+} ; \text {Helper plasmid for } \\
\text { conjugation }\end{array}$ & $\begin{array}{l}\text { (Figurski \& } \\
\text { Helinski, } \\
\text { 1979) }\end{array}$ \\
\hline pRK2013 & $\begin{array}{l}\mathrm{Km}^{\mathrm{R}} \text {; colE1 tra+ RK2 } \mathrm{mob}^{+} ; \text {Helper plasmid for } \\
\text { conjugation }\end{array}$ & $\begin{array}{c}\text { (Finan, et al., } \\
1986)\end{array}$ \\
\hline pMMB67EH-Gm & $\begin{array}{l}\mathrm{Gm}^{\mathrm{R}} \text {; IncQ, RSF1010, lacl }{ }^{q} \mathrm{P}_{\text {tac }} \text { expression } \\
\text { vector with ampR::aacC1 insertion at Dral }\end{array}$ & $\begin{array}{l}\text { (Furste, et } \\
\text { al., 1986) }\end{array}$ \\
\hline pOC19 & $\begin{array}{l}\text { GmR; pMMB67EHGM containing 933-bp EcoRI- } \\
\text { BamHI ampR-His6 fragment from pOC11 }\end{array}$ & $\begin{array}{l}\text { (Caille, et al., } \\
\text { 2014) }\end{array}$ \\
\hline pMS402 & $\begin{array}{l}\mathrm{Km}^{\mathrm{R}}, \mathrm{Tc}^{\mathrm{R}} \text { Expression reporter plasmid carrying } \\
\text { the promoter less luxCDABE; }\end{array}$ & $\begin{array}{l}\text { (Olsen, et al., } \\
\text { 1982, Duan, } \\
\text { et al., 2003) }\end{array}$ \\
\hline $\operatorname{mini}-\mathrm{CTX}$ lux & $\begin{array}{l}\mathrm{Tc}^{\mathrm{R}}, l u \times C D A B E \text { based reporter vector with site } \\
\text { specific integration at attB site in } P \text {. aeruginosa } \\
\text { chromosome }\end{array}$ & $\begin{array}{l}\text { (Becher \& } \\
\text { Schweizer, } \\
\text { 2000) }\end{array}$ \\
\hline pCDS101 & $\begin{array}{l}\text { Modified MINI-CTX with phzA1 promoter } \\
\text { directionally cloned upstream of the luciferase } \\
\text { operon }\end{array}$ & $\begin{array}{l}\text { Personal } \\
\text { Communicati } \\
\text { on with } \\
\text { Surette lab }\end{array}$ \\
\hline pHK478 & $\begin{array}{l}\text { Pampc with Xho1-BamH1 sites, directionally } \\
\text { cloned upstream of the luciferase operon in } \\
\text { pMS402. }\end{array}$ & This study \\
\hline pHK502 & $\begin{array}{l}\text { 8KB Pac1 digest from pCDS101 containing } \\
\text { sequence for integrase mediated recombination } \\
\text { with PAC1 digested pHK478 containing } \mathrm{P}_{\text {ampC }}\end{array}$ & This study \\
\hline \multicolumn{3}{|l|}{ Primers } \\
\hline $\begin{array}{l}\text { EL04_ampC_forward_ } \\
\text { Xho }\end{array}$ & $\begin{array}{l}\text { 5'- } \\
\text { ACACTCGAGAGGAGATATACCATGGATTCC } \\
\text { CGTTTGC-3' }\end{array}$ & This study \\
\hline $\begin{array}{l}\text { EL05_ampC_reverse_ } \\
\text { BamH1 }\end{array}$ & 5'-GGATCCTCAACCCAGAGGCGGCG-3' & This study \\
\hline
\end{tabular}



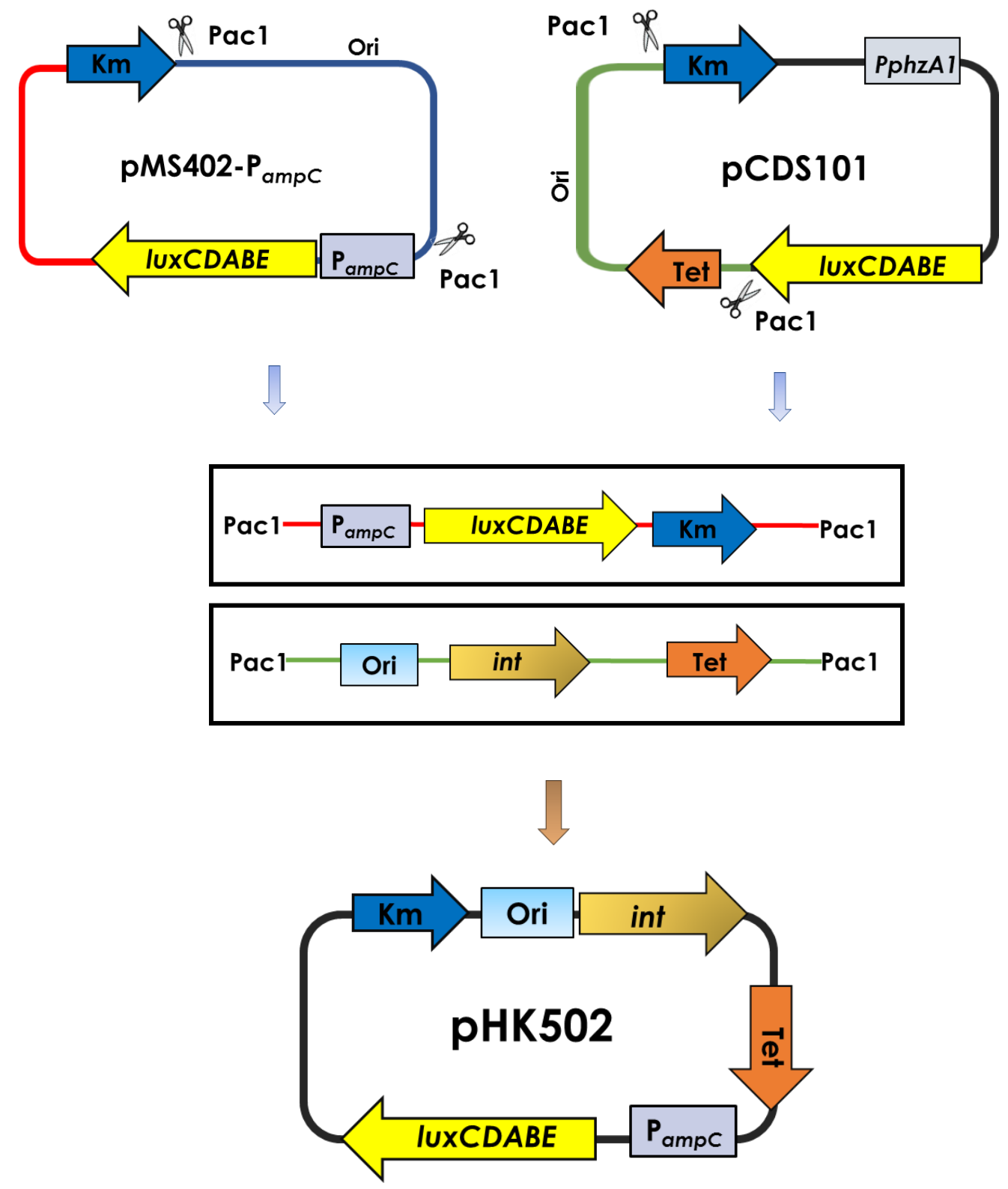

Figure 5.2. Construction of PAO1:: $\mathrm{P}_{a m p c}-\mathrm{lux}$. The strategy is to construct reporters that fuse promoters under direct regulation with AmpR. AmpR has been shown to bind to promoter region of AmpC. From the first plasmid pMS402, the region comprising of ampC promoter with the lux cassette and the marker for kanamycin was digested using Pac1. From the second plasmid, pCDS101- a derivative of mini-ctx lux plasmid, the region comprising of sequences required for integration in the Pseudomonas genome is digested with Pac1. These two fragments are ligated and electroporated into $E$. coli. The resulting plasmid contains the $a m p C$ promoter upstream of a promoterless Lux cassette and is integrated into Pseudomonas genome following triparental mating. 
5.3.5. Luciferase Assay of reporter strains. The reporter strains $P A O 1$ attB:: $P_{a m p c}-l u x$,

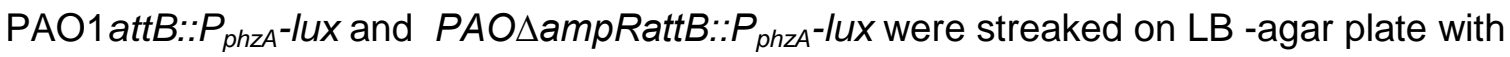
antibiotics. Overnight grown stocks were resuspended in fresh $L B$ to an $\mathrm{OD}_{600}$ of 1 . The bacteria were then re-diluted to an $\mathrm{OD}_{600}$ of 0.02 and grown in $\mathrm{LB}$ until $\mathrm{OD}_{600}$ 0.2. For PAO1 attB:: $\mathrm{P}_{\text {ampc }}-l u x$, the culture was divided into two flasks where one was treated with sub-inhibitory concertation of imipenem $(0.1 \mu \mathrm{g} / \mathrm{ml})$. The cultures were distributed into 96 well plates. This was then placed in the Biotek Synergy plate reader. The initial $\mathrm{OD}_{600}$ and luminescence values was recorded in kinetic mode over three hours at which time the highest levels of luminescence were reached. The relative luminescence units (RLU) were normalized to cell number using $\mathrm{OD}_{600}$. $Z^{\prime}$ value of the assay was compared with the values for positive and negative controls, which in the case of PAOattB:: $\mathrm{P}_{a m p c}-\mathrm{lux}$ was the induced and uninduced cultures. For PAOattB::: $P_{\text {phzA }}-l u x$, negative control was PAO $\triangle a m p R a t t B:: P_{p h z A}-l u x$.

5.3.6. Primary screen with compounds from Torrey Pines. The primary screen was done using compounds from Torrey Pines. The initial screen was done by measuring the luciferase activity of $\mathrm{P}_{\text {ampc }}-\mathrm{lux}$ and $\mathrm{P}_{\text {phzA } 1} / \mathrm{lux}$ and the compounds. The growth $\left(\mathrm{OD}_{600}\right)$ was measured. The respective controls for each condition will be maintained, for e.g. wells without any small molecule compound were kept. The Torrey Pines Institute chemical library contains around 30 million compounds based upon 40 different molecular scaffolds (Nefzi, et al., 2004). The scaffolds are arranged according to positional scanning format and scaffold ranking, which increases chances of identifying an active compound. The library contains approximately 8000 samples which cover over 30 million compounds. These libraries have been used for drug discovery process as 
when compared to other libraries these have the advantage of being able to screen a large number of compounds at the same time (Lopez-Vallejo, et al., 2012). The compounds that show a significant amount of decrease in the luciferase activity and no significant change in $\mathrm{OD}_{600}$, will be considered for further screens.

5.3.7 Statistical analysis. In the primary screen with compounds from Torrey Pines, a $20 \%$ decrease in luciferase activity considering the positive control as $100 \%$ and the negative control as $0 \%$ were selected. To assess the assay quality, $z$ ' values were quantified in PAOattB:: $\mathrm{P}_{\text {amp }}$-lux under induction and negative controls $\mathrm{PAOattB:: \textrm {P } _ { \text { ampC} }}{ }^{-}$ lux without induction using previously described equation (Zhang, et al., 1999). For PAOattB:: $\mathrm{P}_{\text {phzA }}$-lux, the reporter fusion in ampR deletion was considered as negative control. Z' values were considered significant above 0.7 .

\subsection{Results}

5.4.1 AmpR is localized in the membrane. Our previous analysis performed bioinformatically found that $\mathrm{AmpR}$ has a transmembrane domain and is membrane associated (Caille, et al., 2014). This was further proven through LacZ-PhoA studies that AmpR has a periplasmic domain (Caille, et al., 2014). Localization was further investigated using protease protection experiments. This showed that incubation with proteinase $\mathrm{K}$ resulted in degradation of AmpR found in spheroplasts (Caille, et al., 2014). In this research, we probed the localization of $A m p R$ again using ultracentrifugation which show that AmpR localizes in the membrane. The membrane fractions were 
confirmed further using enzyme assays. Lactate dehydrogenase, an enzyme found specifically in the membrane was associated with the AmpR fractions.
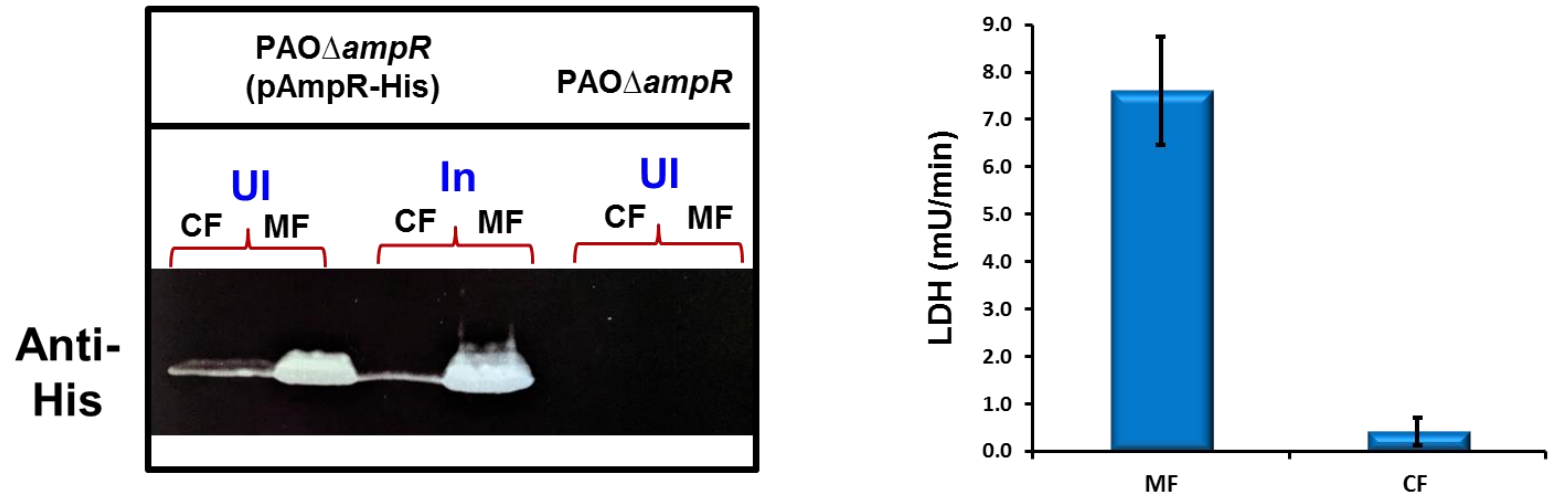

Figure 5.3. AmpR fractionates in the membrane. Fractionation studies using PAOampR (pAmpR-His) found that $P$. aeruginosa AmpR resides in the membrane. The fractions were further confirmed by enzyme analysis. Lactate dehydrogenase is found primarily in the inner membrane in bacteria. Fractions containing AmpR were consistently found to have a higher level of this enzyme when compared with the cytoplasmic fraction.(CF-cytoplasmic fraction, MF- Membrane fraction, UI-Uinduced, In-Induced)

\subsubsection{Gly102Glu leads to structural instability whereas Asp135Asn stabilizes}

AmpR dimer. Previous experiments have indicated that Glycine 102 and Aspartate 135 were critical residues for AmpR to act as an activator of AmpC $\beta$-lactamase. Substitution of Aspartate 135 with Asparagine led to an inducer-independent hyper-expression of $\mathrm{P}_{\text {ampc }}($ Caille, et al., 2014). It was presumed that this may be due to structural stabilization of the dimer. In the dimer model, residue 135 in the dimer is also located adjacent to one another (Figure 5.4A). Hence, replacing charged amino acid Aspartate with uncharged asparagine leads to structure stabilization of the AmpR dimer model. As this mutation results in constitutive hyper-expression of $\mathrm{AmpC}$, it further supports our hypothesis that AmpR is active as a dimer. 


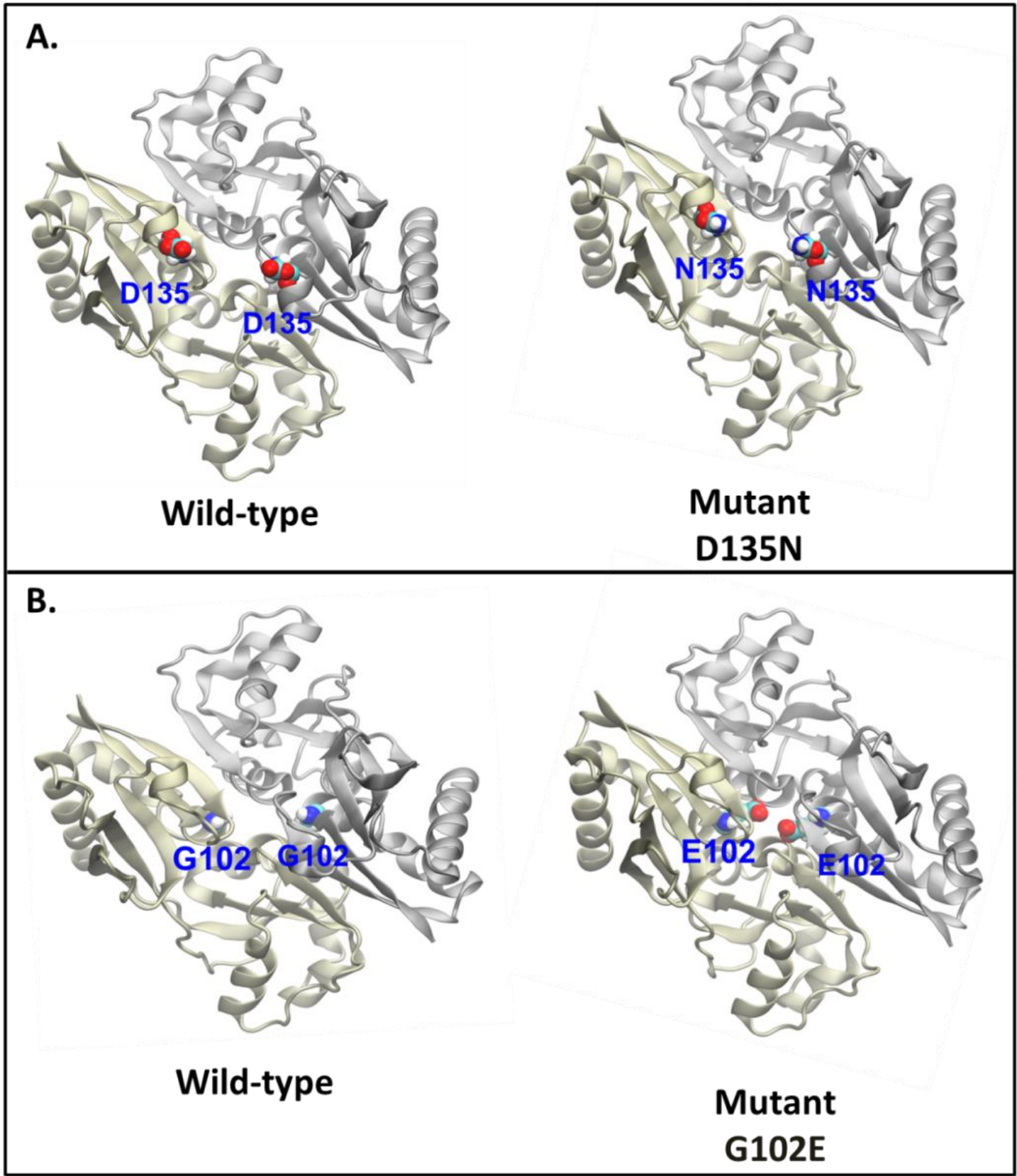

Figure 5. 4. Protein models of AmpR mutants. In panel A replacement of charged Aspartate with Asparagine leads to stabilization of AmpR dimer. This explains the constitutive high-level expression of AmpC. In Panel B, two negative charged E102 residues in the dimer interface will destabilize the dimer due to strong electrostatic repulsion. This explains the loss of phenotype seen in the G102E mutant. 
Previous literature in Citrobacter freundii has shown that substituting Glycine 102 with glutamate resulted in a constitutive hyper-expression of AmpC (Bartowsky \& Normark, 1993). This was however not the case in $P$. aeruginosa, where substitution resulted in an inability to induce AmpC (Caille, et al., 2014). When the structural model was looked at, it was observed that upon AmpR dimerization, the residues 102 of each monomer are closely oriented (Figure 5.4 B). As Glutamate is a charged amino acid, it resulted in the repelling of monomers due to strong repulsive electrostatic interactions and leads to a structural destabilization of the dimer formation, hence leading to a noninducible phenotype for AmpC. Such destabilization and the detailed atomistic interactions can be explored by performing molecular dynamics simulations.

5.4.3 Validation of reporter constructs. The reporters constructed were validated without compounds to check for luciferase activity. For validation of PAOattB::P $\mathrm{P}_{\text {ampc-lux, }}$ the luciferase activity generated over six hours was monitored with a reading taken at every hour. Imipenem which has been demonstrated to be a strong inducer of AmpC activity was used as an inducer. It was found that the activity was highest at 3 hours. The luciferase activity of the PphzA1-lux was compared with and without induction in both PAOI and PAOampR background. It was found that deletion of AmpR abolished luciferase expression, validating its use as a negative control for the reporter PAO1PphzA1-lux (Figure 5.5). 


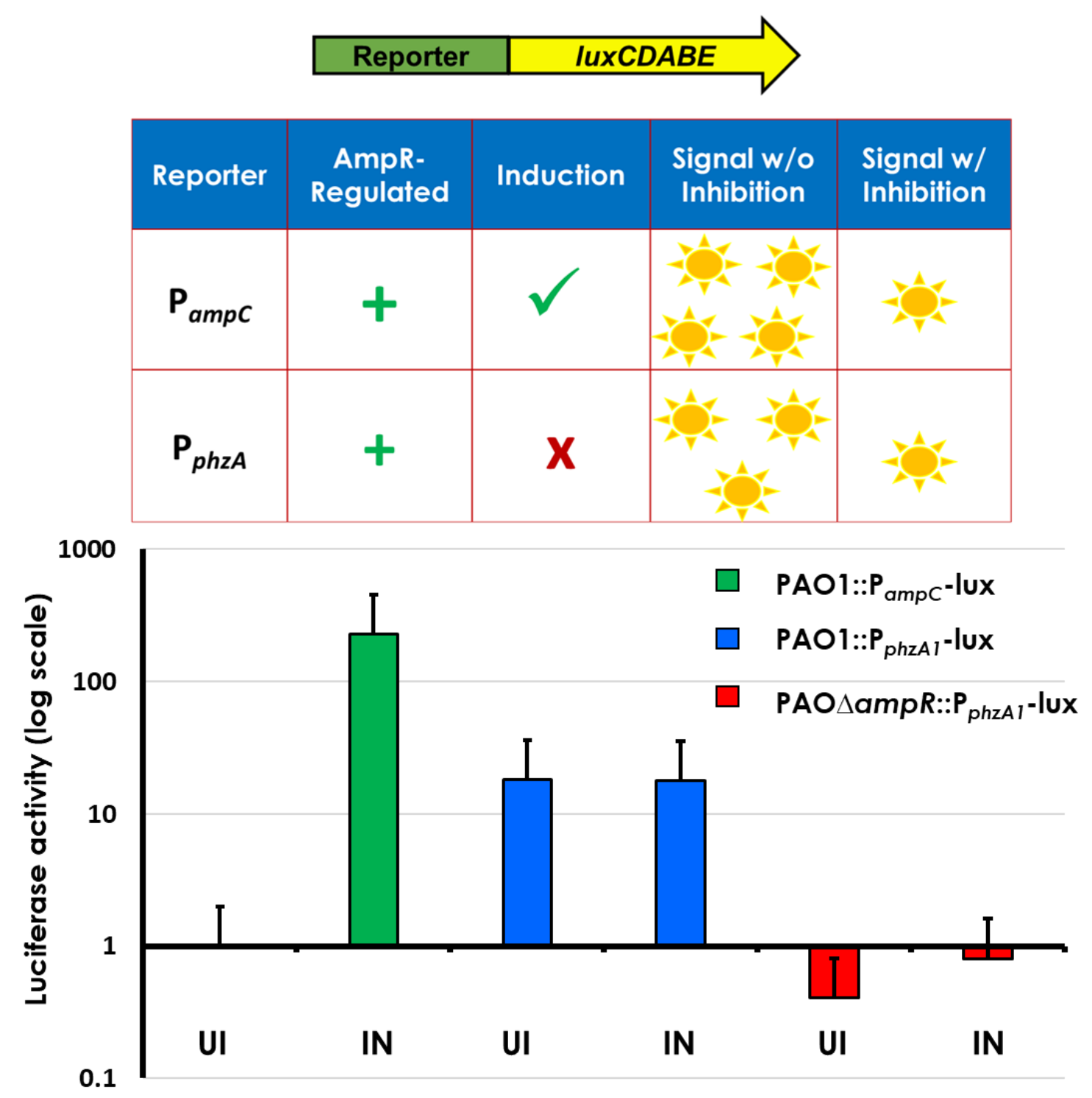

Figure 5.5. Luciferase activity in the reporter constructs. The luciferase activities in the reporters were checked with and without induction. PphzA1 is regulated by AmpR in the absence of induction; as can be seen here. The luciferase detected in the

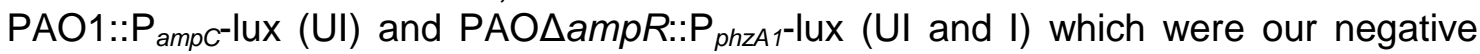
controls for each reporter were similar. All values are normalized to PAO::P $\mathrm{P}_{a m p c}$-lux uninduced. 
5.4.4 Primary screen of Torrey Pines compounds. After validating our reporters with respective controls, $\mathrm{PAO}:: \mathrm{P}_{a m p c}-\mathrm{lux}\left(\mathrm{Z}^{\prime}=0.88\right)$ was used to screen the scaffold-based combinatorial library available at Torrey Pines. The scaffold-based design of the library allows screening for numerous compounds at once. As the compounds were dissolved in Dimethyl formamide (DMF), an initial screening for solvent tolerance was done to determine growth at increasing concentrations of DMF.

Primary screen with the compounds provided a few hits. The analysis of the primary compounds was done at dilutions of $5 x$ and $10 x$, monitoring the bacterial growth at the same time as luciferase (Figure 5.7). Finally, compounds were used at $10 x$ dilution as that was found to affect growth the least. After normalizing the luciferase activity with growth, compounds that showed $20 \%$ decrease in luciferase as compared to the positive control were considered for a secondary screen (Figure 5.8). It was also found that the positive hits could be structurally divided into two groups of pyrrolidine-based and guanidine-based compounds. These were then targeted for a secondary screen using

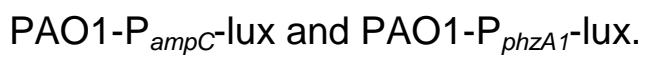

\subsection{Discussion}

Genomic and proteomic data from our lab demonstrated the extensive role of AmpR in virulence and antibiotic resistance in P. aeruginosa (Balasubramanian, et al., 2012, Balasubramanian, et al., 2014, Kumari, et al., 2014). Much of this data was further confirmed by phenotypic assays (Balasubramanian, et al., 2012). This research explored further into AmpR and its localization in P. aeruginosa. The presence of AmpR in the membrane is interesting as transcriptional regulators are typically known to exist in the 


\begin{tabular}{|c|c|c|c|c|c|c|c|c|c|}
\hline \multicolumn{10}{|c|}{ \% Reduction in signal : $5 x$ dilution } \\
\hline & \multicolumn{7}{|c|}{ Compounds } & & \\
\hline & 1 & 2 & 3 & 4 & 5 & 6 & 7 & & \\
\hline$A$ & 47 & 41 & -5 & -6 & 8 & 7 & 12 & & \\
\hline B & 39 & 19 & -13 & 26 & 33 & 28 & 5 & \multirow{7}{*}{ DMF } & \\
\hline C & 32 & 13 & 28 & -53 & 23 & 28 & -2 & & \\
\hline D & 24 & 19 & -35 & -33 & 2 & 35 & -16 & & \\
\hline $\mathrm{E}$ & 15 & 30 & -24 & 11 & 24 & -13 & -20 & & $>40 \%$ \\
\hline $\mathrm{F}$ & 25 & 26 & 27 & 23 & 27 & -20 & -17 & & $\geq 30 \%$ \\
\hline G & 24 & 14 & 27 & 18 & 23 & 2 & -28 & & Negative \\
\hline $\mathrm{H}$ & 22 & 14 & 17 & 94 & -34 & -18 & -2 & & Affected growth \\
\hline \multicolumn{10}{|c|}{$\%$ Reduction in signal : 10x dilution } \\
\hline & \multicolumn{7}{|c|}{ Compounds } & & \\
\hline & 1 & 2 & 3 & 4 & 5 & 6 & 7 & & \\
\hline $\mathrm{A}$ & 58 & 54 & 25 & 22 & 18 & 18 & 25 & & \\
\hline $\mathrm{B}$ & 53 & 44 & 27 & 19 & 28 & 22 & 9 & \multirow{7}{*}{ DMF } & \\
\hline $\mathrm{C}$ & 49 & 27 & 17 & -4 & 31 & 34 & 8 & & \\
\hline $\mathrm{D}$ & 33 & 37 & 21 & -13 & 27 & 22 & 12 & & $>50 \%$ \\
\hline $\mathrm{E}$ & 27 & 29 & -3 & 20 & 24 & 4 & 2 & & $>40 \%$ \\
\hline $\mathrm{F}$ & 35 & 20 & 34 & 33 & 17 & -7 & 2 & & $>30 \%$ \\
\hline $\mathbf{G}$ & 21 & 19 & 23 & 9 & 21 & -9 & 4 & & Negative \\
\hline $\mathrm{H}$ & 32 & 24 & 28 & -32 & -3 & -10 & 4 & & Affected growth \\
\hline
\end{tabular}

Figure 5. 6 Optimizing compound concentrations for screening. Initial screening of compounds was done in both a $5 x$ and a $10 x$ dilution of compounds. $5 x$ dilution with many compounds were found to inhibit growth with no significant effect on luciferase activity. Hence, compounds were used at $10 \mathrm{x}$ dilutions for all subsequent assays. 


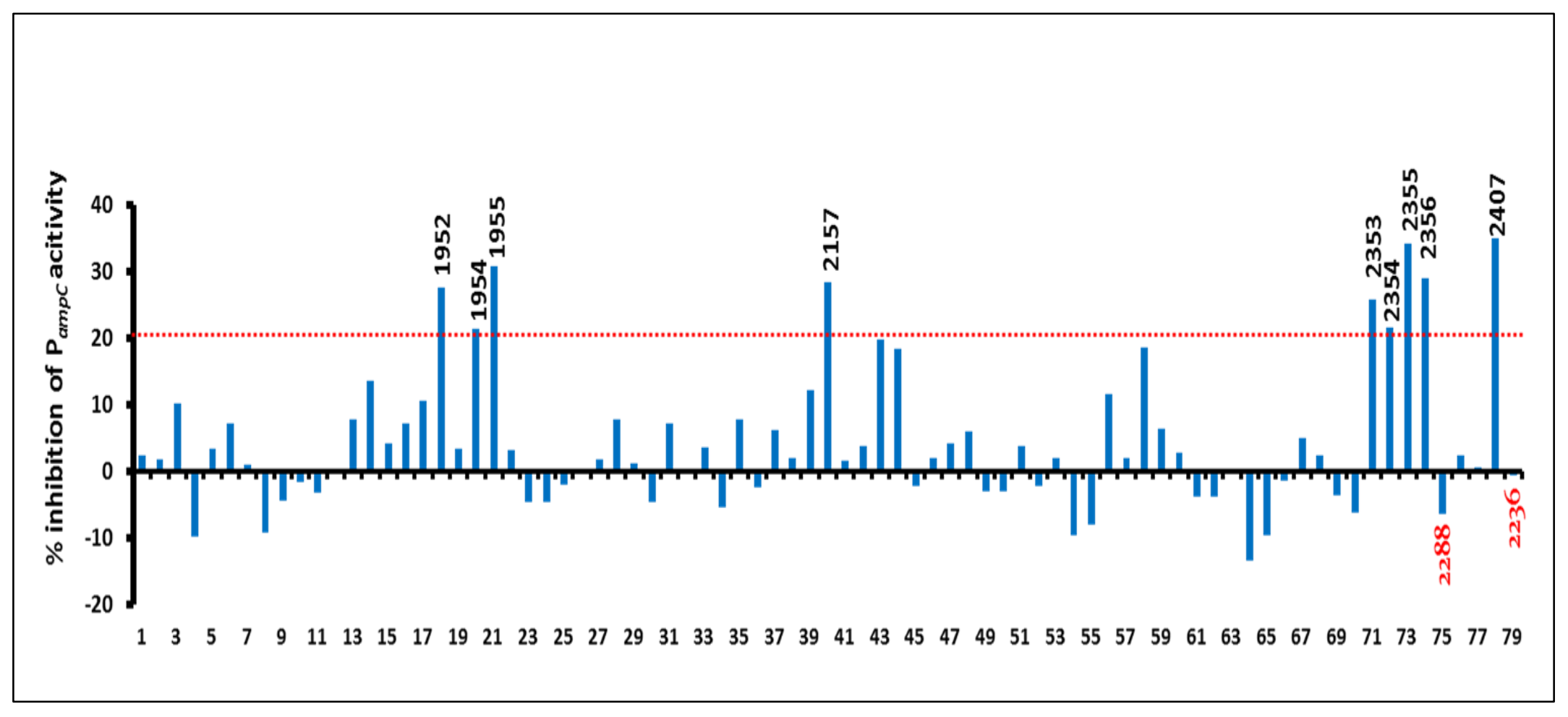

Figure 5.7. Primary screening of scaffold ranking library.Primary screening of the scaffold ranking library using compounds at a concentration of $5 \mu \mathrm{M}$. Samples that inhibited luciferase activity $>20 \%$ when compared to positive control (PAO1:: $\mathrm{P}_{a m p c}$-lux, induced) were further analyzed for secondary screening. 
cytoplasm. However, previous models of transcriptional regulator in Rhizobium melliloti showed the presence of membrane-associated transcription factors, NodD (Schlaman, et al., 1989). The location of AmpR in the membrane also has implications towards the identification or localization of the AmpR effectors (Lee, et al., 2016). The AmpR effectors may be located to the periplasm, in addition to being found in the cytoplasm.

Also, different studies have shown that AmpR exists as a dimer and a tetramer (Vadlamani, et al., 2015, Dik, et al., 2017). Through mutational analyses of selected residues in AmpR, our previous study identified phenotypic differences with regards to AmpC expression (Caille, et al., 2014). The role of the residues was further confirmed with our research here which shows that AmpR is active as a dimer as mutations of G102Glu results in de-stabilization of the dimer and Asp135Asn leads to stabilization of the dimer. This occurs due to the gain or loss of charged amino acids, which results in increased and decreased electrostatic repulsion, respectively.

It is known that AmpR along with other members of the cell-wall recycling pathway regulate $\beta$-lactam resistance (Johnson, et al., 2013). Our principle for advocating AmpR as a novel antibacterial target was to identify inhibitors that reduce virulence as well as prevent induction of AmpC $\beta$-lactamase thereby helping in a synergistic action with $\beta$ lactams. We also developed a novel cell-based screening with reporters using luciferase. Previous knowledge regarding the $A m p R$ regulon led us to use $A m p C$ promoter for the constructing the reporter screen (Balasubramanian, et al., 2012). In addition, other promoters known to be regulated by AmpR such as PhzA1 and MexE were developed for counter-screening (Balasubramanian, et al., 2012). Transcriptional fusions were generated with these promoters with the luxABCDE operon. The primary screens identified compounds that belonged to two distinct chemical compounds, 
pyrrolidine and guanidine-based compounds. Using this information, we can identify compounds with similar structures that may specifically inhibit AmpR. Secondary screening of the compounds identified so far can be done with the second reporter system $\mathrm{P}_{\text {phzA1. }}$. Furthermore, as the effectors of AmpR have been identified in our previous studies, our reporter system can be used for a more targeted approach to identify muramyl-peptide analogs inhibiting AmpR.

5.6 Acknowledgements. The structural modeling was done in collaboration with Dr. Prem Chapagain and Dr. Jeevan GC at Department of Physics with College of Arts, Sciences and Education at Florida International University. Th screening for smallmolecules was done in collaboration with Torrey Pines Institute, Florida. We are grateful to Dr. Mark Guillanotti for providing us the compounds as well as intellectual input and discussions. 
Chapter 6

Overview, Summary and Future Directions 


\subsection{Overview}

Pseudomonas aeruginosa is a Gram-negative, metabolically-versatile, ubiquitous bacterium that is known for causing life-threatening infections in immunocompromised people (Eng, et al., 1986, Kiehn, 1989). Commonly identified as a soil microbe, the incredible ability of these bacteria to adapt and thrive in diverse environments has contributed to its success as an important pathogen (Govan \& Harris, 1986, Silby, et al., 2011, Marvig, et al., 2015, Winstanley, et al., 2016). Although rare, P. aeruginosa can cause infections such as folliculitis, otitis externa, and keratitis in healthy individuals (Ramphal, et al., 1981, Mena \& Gerba, 2009). In addition, P. aeruginosa is one of the main pathogens that account for most of the hospital-acquired infections (Pittet, et al., 1999, Cadro, 2004). Other serious infections in patients include burn-wound or postsurgery which leads to bacteremia that may proceed to fatal sepsis (McManus, et al., 1985, Osmon, et al., 2004, Yildirim, et al., 2005). Age, prolonged intubation, chemotherapy, HIV AIDS as well as the use of broad spectrum antibiotics can result in lowering immunity leading to the manifestation of $P$. aeruginosa infection (Kielhofner, et al., 1992, Caselli, et al., 2010). A notable disease that is almost always associated with P. aeruginosa is cystic fibrosis (Emerson, et al., 2002, Marshall, et al., 2016).

Complicating $P$. aeruginosa infections is its remarkable intrinsic ability to thwart multiple antibiotics (Lambert, 2002, Livermore, 2002). This single species harbors numerous resistance mechanisms which include impermeability of the outer membrane, active efflux of small molecules via efflux pumps, modification of the antibiotic target rendering them unrecognizable and the production of hydrolytic enzymes specifically, towards a group of drugs often used, the $\beta$-lactams (Hancock, 1998, Livermore, 2002, Fisher, et al., 2005). 
A major mechanism of resistance specific to $\beta$-lactams is the expression of hydrolytic enzymes that cleave the $\beta$-lactam ring rendering them ineffective. P. aeruginosa harbors chromosomally encoded $\beta$-lactamases. However, it easily acquires genes via horizontal transfer as well as plasmid, transposon or integron encoded (Zhao \& Hu, 2010, Breidenstein, et al., 2011). These enzymes include the Class A and D extended spectrum $\beta$-lactamases as well as the Class B carbapenemases (Paterson \& Bonomo, 2005, Poirel, et al., 2010, Poole, 2011). In addition to these, three $\beta$ lactamases are encoded on the $P$. aeruginosa genome. This includes PIB-1/PA5542; Class D oxacillinase PoxB/PA5297, formerly known as OXA-50, and the Class C cephalosporinase AmpC/PA4110 (Kong, et al., 2005, Fajardo, et al., 2014, Zincke, et al., 2016). PoxB was recently characterized by our lab as a carbapenemase which leads to resistance, especially upon down-regulation of the porin OprD whereas PIB-1 was found to be a zinc-dependent imipenemase (Fajardo, et al., 2014, Zincke, et al., 2016).

The third chromosomal $\beta$-lactamase, AmpC (PA4110) is well-characterized in $P$. aeruginosa and is known to be regulated by the transcriptional factor AmpR (PA4109) (Lodge, et al., 1993, Kong, et al., 2005). Expression of ampC is inducible in the presence of certain $\beta$-lactams and requires $A m p R$ and its effector, a muropeptide generated by the concerted activity of the proteins in the cell-wall recycling pathway (Jacobs, et al., 1997, Dhar, et al., 2018). Cell-wall recycling is a process that is conserved in both Grampositive and -negative bacteria (Reith \& Mayer, 2011, Johnson, et al., 2013). Recycling of the cell-wall that occurs during growth, division and elongation was eventually linked to its intrinsic role in AmpC induction and hence, $\beta$-lactam resistance (Goodell \& Schwarz, 1985, Lindquist, et al., 1989). The link between the two processes was suggested when it was discovered that the signaling molecules which interact with AmpR were not $\beta$-lactams but were speculated to be the cell-wall fragments generated 
in the presence of $\beta$-lactams (Lindquist, et al., 1989). This hypothesis was confirmed when it was identified that $A m p G$ and $A m p D$ were required for ampC expression in $C$. freundii and E. cloacae and that these genes were also involved in recycling of cell-wall fragments or muropeptides (Korfmann \& Wiedemann, 1988, Korfmann \& Sanders, 1989, Lindquist, et al., 1989, Jacobs, et al., 1994).

Since then cell-wall recycling has been studied mainly in E. coli (Park \& Uehara, 2008). However, to better understand the details of this induction process special focus has been given to pathogens that contain the $a m p R$-ampC region, including members of Enterobacteriaceae and in $P$. aeruginosa (Jacobs, et al., 1994, Balasubramanian, et al., 2015). Many proteins of cell-wall recycling such as the lytic transglycosylases, amidases, permeases, $\mathrm{N}$-acetyl $\beta$-D glucosaminidases, and ligases are involved in the AmpC induction process (Kong, et al., 2010, Dhar, et al., 2018). Among these, in P. aeruginosa mutations of specific ones such as amidases- AmpDh2, AmpDh3 and AmpD; LMM penicillin-binding proteins; $\mathrm{N}$-acetylglucosaminidase NagZ; permease AmpG have been shown to affect AmpC expression (Juan, et al., 2006, Kong, et al., 2010, Johnson, et al., 2013, Ropy, et al., 2015). Many of these enzymes play a seminal role in generating the muropeptide effector of $P$. aeruginosa AmpR as has been entailed in some detail in Chapter 1 (Juan, et al., 2006, Asgarali, et al., 2009, Kong, et al., 2010). However, to date the muropeptides generated in $P$. aeruginosa as well as the identity of the AmpR effector remains elusive.

This dissertation delved into the role of cell-wall recycling intermediates as determinants of $\beta$-lactam resistance in $P$. aeruginosa. This work investigated for the first time muropeptides and their structure in $P$. aeruginosa and identified the specific muropeptides that act as AmpR effectors. In addition, the role of AmpG and AmpP in the 
transport of muropeptides was analyzed in $P$. aeruginosa. We identified disaccharide muropeptides as the substrates for these permeases. The identity of a putative $\mathrm{N}$ acetylglucosaminidase was investigated. Finally, reporters were constructed to screen for small-molecule inhibitors for AmpR.

6.2. Muropeptides in $\boldsymbol{P}$. aeruginosa and AmpR effector. Cell-wall intermediates are often generated when the cell divides. These products are released from the peptidoglycan wall and structurally are composed of variations of sugars and peptides (Glauner, et al., 1988). The sugars include $N$-acetylglucosamine (GlcNAc) and $N$-acetyl muramic acid (MurNAc) and the peptides are composed of L-alanine, $\gamma$-D-glutamine, meso-diaminopimelic acid (m-DAP), and D-alanine (Glauner, et al., 1988). The composition of the PG is conserved mostly across Gram-negative bacteria (Schleifer \& Kandler, 1972, Vollmer \& Holtje, 2004). The main variation found in Gram-positive bacteria is the presence of L-Lysine in place of $m$-DAP as the third amino-acid of the peptide chain (Schleifer \& Kandler, 1972).

In $P$. aeruginosa PAO1 we were able to detect upto 20 distinct muropeptides using liquid chromatography with tandem mass-spectrometry (Chapter 2, Table 2.2). A method to quantitate the muropeptides was developed. For our study, we used $P$. aeruginosa at late-log phase of growth to investigate the muropeptide levels. It is well-appreciated that the PG undergoes structural modifications based upon the phase of bacterial growth. In E. coli it was found that bacteria in the exponential phase of growth had higher crosslinked muropeptides compared to one that is not dividing (Glauner, et al., 1988, Keep, et al., 2006). In vitro activity of PG degradative enzymes such as the lytic transglycosylases on the isolated murein sacculus at log-phase led to a higher level of muropeptide products compared to one obtained at stationary phase (Lee, et al., 2013). In our 
research, a protocol was developed wherein we can isolate and detect muropeptides using LC/MS/MS from bacterial extracts. This work was done in collaboration with Dr. Mobashery's lab at UND. The conditions used for LC/MS were established previously and we were able to identify muropeptides up to picomolar concentration (Lee, et al., 2013). The muropeptides that were generated are the results of numerous enzymes found in the cytoplasm and the periplasm (Chapter 1, Table 1.2). Using synthetic standards of muropeptides available with our collaborator, we were able to quantitate the absolute number of muropeptides (molecule/cell) in the bacterial cell (Chapter 2, Table 2.1).

The total ion chromatogram (TIC) did not reveal any specific muropeptide; hence extracted ion chromatogram was used and muropeptides detected using synthetic muropeptides (Chapter 2, Figure 2.3). The most abundant muropeptide detected was the disaccharide-tetrapeptides ( $\mathrm{N}$-acetylglucosamine- 1, 6 anhydro MurNAc L-Ala-D-Glum-DAP-D-Ala) (Chapter 2, Table 2.1). Disaccharides with varying number of aminoacids were detected; however, pentapeptide which is the full amino acid side chain was present in minute quantities. These were the products of lytic transglycosylase with exolytic activities. So far, P. aeruginosa has shown the presence of eleven LTs all of which have shown to have exolytic activity (Lee, et al., 2017). Monosaccharides such as 1, 6 anhydro MurNAc tri and tetrapeptides were also detected indicating the presence of $\mathrm{N}$-acetylglucosaminidase. Identification of 1, 6 anhydroMurNAc peptides in the periplasm led us to postulate the presence of a second $\mathrm{N}$ - acetylglucosaminidase, FlgJ. The role of this enzyme in cell-wall recycling and antibiotic resistance is the focus of chapter 4.

Presence of a cell-wall active drugs such as $\beta$-lactams in the environment trigger the generation of certain muropeptides that act as AmpR effectors (Jacobs, et al., 1997). 
The bacteria grown in LB media without $\beta$-lactam were compared with the bacteria exposed to non-lethal concentration of $\beta$-lactam (cefoxitin). Cefoxitin is a strong inducer of $\beta$-lactamase and more stable to its hydrolytic activity (Jacoby, 2009). Overall, it was found that the levels of muropeptides upon $\beta$ - lactam induction were decreased (Chapter 2, Table 2.1). Relative levels of individual muropeptides were similar; however, one discriminatory feature was consistently increased presence of pentapeptides upon induction. There was a 46-fold induction of $N$-acetylglucosamine 1, 6 anhydro $N$-acetyl muramic acid pentapeptide in the presence of $\beta$-lactam stress and the 1,6 N-acetyl muramic acid pentapeptide was detected only upon induction. This increase probably occurs as cefoxitin acts by targeting PBPs. Upon binding with low molecular mass PBPs (LMM PBP), both the transpeptidase and endopeptidase functions of these enzymes are lost, and there is an increase of their specific substrates (Lee, et al., 2015). In such cases, the pentapeptide containing muropeptides (substrates of LMM-PBP) may increase relatively in the cytosol. As there was a significant increase in the levels of the pentapeptides upon induction, it was further analyzed if these muropeptides act as AmpR effector. A $\beta$-lactamase assay with synthetic constructs indeed revealed that these muropeptides were capable of inducing $\beta$-lactamase expression (Chapter 2, Figure 2.3). A subsequent study also confirmed our findings by clearly elucidating the binding and interaction of these muropeptides with AmpR (Dik, et al., 2017).

$P$. aeruginosa cell-wall recycling has been found to deviate from that of Enterobacteriaceae. $P$. aeruginosa has multiple amidases whose down regulation is required for the maximal $\beta$-lactamase expression (Juan, et al., 2006). It has two inner membrane permeases hypothesized to play a role in cell-wall recycling in $P$. aeruginosa (Kong, et al., 2010). AmpR itself in $P$. aeruginosa has been found to have a more extensive regulon when compared to its counterpart in $E$. cloacae and $C$. freundii 
(Balasubramanian, et al., 2015). Previous research in $C$. freundii had shown that the 1, 6 anhydro muramic acid tripeptide can act as the AmpR effector (Jacobs, et al., 1994). This was however not found to be the case in $P$. aeruginosa (Chapter 2, Table 2.2).

6. 3. AmpG and AmpP play a role in cell wall recycling. $P$. aeruginosa has two $E$. coli AmpG homologs, PA4393/AmpG and PA4218/AmpP. The MIC of certain $\beta$-lactams was decreased in PAO ampG (Chapter 3, Table 3.1). This decrease in MIC can be explained by the diminished levels of $\beta$-lactamase expressed in PAO $\Delta a m p G$ (Chapter 3, Figure 3.2). It is known that AmpG is conserved across many Gram-negative bacteria and plays a role in muropeptide transport ( $\mathrm{Li}$, et al., 2016, Perley-Robertson, et al., 2016). Based on our previous research and significant homology of AmpP with E. coli AmpG, we also hypothesized that AmpP plays a role in muropeptide transport, probably by shuttling muropeptides from the cytoplasm into the periplasm (Kong, et al., 2010). When the muropeptides levels were compared between the different fractions, it was found that disaccharides accumulate in the periplasm and cytoplasm respectively in $\mathrm{PAO} \triangle a m p G$ and PAO $\triangle a m p P$ (Figure 3.8). This suggests that these permeases play a role in transporting disaccharide-peptides into and out of the cytoplasm. When the periplasmic extract of muropeptides was isolated, mostly disaccharides were detected in all strains as compared to the whole cell preparation (Figure 3.5). This is expected as the enzyme $\mathrm{N}$-acetylglucosaminidase that cleaves the two sugar units GlcNAc and MurNAc, is known to be cytosolic. However, a minor amount of monosaccharides were found in all the periplasmic preparation, indicating the presence of a putative periplasmic $\mathrm{N}$-acetylglucosaminidase (Figure 3.6). Larger peptides that included the cross-linked and the tetrasaccharide peptides were also detected at higher levels in the preparation used 
for whole cell extracts (Figure 3.7). This probably reflects the type of preparatory method used for isolating whole-cell muropeptides, as it involves sonication whereas the periplasmic preparation is done by a gentler osmotic shock. To summarize, our analyses showed the presence of muropeptides in both whole-cell as well as periplasmic preparation. Distinctions between mutant strains were made at relative levels of individual muropeptides. Increased levels of periplasmic disaccharide-muropeptides were found in ampP and double-deletion strains whereas decreased levels were seen in ampG deletion strains. This indicates that AmpP can transport muropeptides from the cytoplasm into the periplasm. This method of muropeptide analysis can be used to analyze relative levels or trends of individual muropeptide or to detect presence or absence of specific muropeptides between different strains.

6.4. Role of PA1085 in P. aeruginosa. PA1085 is the fifth gene of a seven-gene operon in $P$. aeruginosa and is involved in flagellar biosynthesis and assembly. $P$. aeruginosa is a monotrichous organism and motility is one of its primary virulence factors (Gellatly \& Hancock, 2013). In our previous analyses of muropeptides, we were able to consistently detect monosaccharide muropeptides in the periplasmic space indicating that there may be a periplasmic enzyme that generates these products from the peptidoglycan (Lee, et al., 2016). The only characterized $N$-acetylglucosaminidase in $P$. aeruginosa is NagZ (PA3005) which is cytosolic. It was found that a periplasmic homolog of this enzyme as characterized in $S$. enterica known as flgJ. This enzyme is part of the flagellar operon and is hypothesized to hydrolyze the PG and enables the insertion of the flagellar units into the PG and its extrusion though the outer membrane into the extracellular milieu (Kerridge, et al., 1962, Herlihey, et al., 2014). In-silico analysis of the $P$. aeruginosa 
PAO1 genome led to the identification of PA1085. There was 31\% homology between FlgJ of $P$. aeruginosa and $S$. enterica. Sequence alignment also showed that the residues that are required for this activity were conserved between $P$. aeruginosa and $S$. enterica FlgJ. In an effort to understand the role of flgJ in $P$. aeruginosa, an in-frame deletion mutant was constructed. It was found that with the loss of flgJ, swimming activity was affected whereas swarming and twitching were not (Figure 4.3). Motility also influences other phenotypes such as biofilm formation (O'Toole \& Kolter, 1998). Loss of flgJ resulted in an increase in biofilm over 48 hours (Figure 4.4). NagZ (PA3005) in $P$. aeruginosa is required for maximal $\beta$-lactamase expression and its absence leads to a decrease in MIC towards selected antibiotics. (Asgarali, et al., 2009). However, deletion of flgJ did not lead to any changes in the MIC of antibiotics (Figure 4.5). The levels of $\mathrm{N}$ acetylglucosaminidase in whole-cell extracts were also unchanged with the loss of FlgJ (Figure 4.6).

6.5. Screening of small molecule library for AmpR inhibitors. The premise for this aim is built upon the previous research which demonstrated that AmpR has an expansive regulon in $P$. aeruginosa and is connected to various virulence and antibiotic resistance regulatory networks (Balasubramanian, et al., 2012). For the identification of AmpR inhibitors, we developed a reporter assay using the luciferase operon luxCDABE fused to promoters known to be regulated by AmpR (Becher \& Schweizer, 2000). The

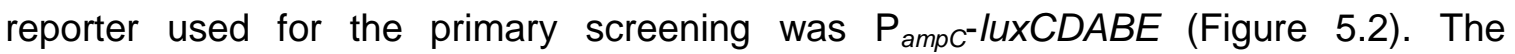
luciferase assay was used as it is a one-step assay which does not require any manipulation such as addition of exogenous substrate or an inducer (Craney, et al., 2007). Using this system, a primary screening was initiated with the scaffold based 
combinatorial library available at Torrey Pines Institute that allows the screening of millions of compounds arranged around 79 core scaffolds. The primary screening of the library was done at a final concentration of $5 \mu \mathrm{M}$ of the compounds, monitoring the growth of bacteria at the same time. Samples that inhibited $P_{a m p c}$ activities by $20-40 \%$ when compared to the positive control were analyzed further (Figure 5.8). Based on the core structures, the samples that showed inhibition were either guanidine or pyrrolidinebased. This corroborates with a recent study which used this chemical library and identified a guanidine based compound with anti-bacterial activity against all the ESKAPE (E-Enterococcus faecium, S-Staphylococcus aureus, K-Klebsiella pneumoniae, A-Acinetobacter baumanii, P- Pseudomonas aeruginosa, EEnterobacter species) pathogens (Fleeman, et al., 2015). Pyrrolidine-based compounds synthesized in-house have previously been demonstrated to be inhibitors of quorum sensing. Our previous research also identified $A m p R$ as a regulator of $Q S$. It would be interesting to further determine if the pyrrolidine compounds available from Torrey-Pines available are AmpR-specific and QS inhibitors.

\subsection{Future directions}

This research is one of the first reports of the muropeptides identified in $P$. aeruginosa using LC/MS/MS. It is also the first report that demonstrated a method to quantitate muropeptides without radioactive-tagging as well as reported on the structural nature of the individual muropeptides. We studied the effect of $\beta$-lactams on muropeptide levels and the identity of the AmpR effector. This research led to the advent of many unanswered questions. The gaps in knowledge have been addressed in sections. The first part deals with the study of muropeptides in $P$. aeruginosa and its 
relevance to antibiotic resistance. Following are further ideas that can be pursued to illustrate better the relevance of muropeptides in $P$. aeruginosa.

I. Investigate muropeptides in clinical-strains and correlate with virulence in host.

Muropeptides generated during cell-wall recycling and synthesis may differ among the different $P$. aeruginosa strains. As muropeptides are significant in maintaining virulence, it would be pertinent to investigate their levels in clinical isolates. In this research we used PAO1 and its isogenic mutants for all our investigations (Lee, et al., 2016). Future studies should elucidate the muropeptide levels, found intracellularly and the muropeptides released into media from $P$. aeruginosa clinical-isolates. A correlation between the released muropeptides and disease phenotypes has been demonstrated in pathogens such as Bordetella pertussis, Neisseria gonorrhea, and N. meningitides (Cookson, et al., 1989, Chan \& Dillard, 2016). Such an investigation can be undertaken using clinical isolates of $P$. aeruginosa and the trend of muropeptides generated therein compared with the lab strain PAO1. The protocol developed in the current study to isolate and quantitate muropeptides can be used for the different strains. Future studies can determine whether the structural nuances, levels, and composition of muropeptides alter the pathogenesis of the organism and the interaction with host receptors. This can be done by incubating an immune cell line such as the Mono Mac 6 (MM6) cells or the human embryonic kidney cell line (HEK293T) with muropeptiedes followed by evaluation of the gene expression levels of Tumor Necrosis Factor- $\alpha$ (TNF- $\alpha$ ) (Wolfert, et al., 2007). TNF- $\alpha$ is known to be stimulated when exposed to muropeptides (Wolfert, et al., 2002). Alternatively, stimlaution of the NOD receptors can be investigated in HEK-Blue hNOD1 and HEK-Blue hNOD2 reporter cell lines (Torrens, et al., 2017). 
II. The mechanistic details of the interaction of muropeptide with receptors of the immune system can be investigated.

It is well appreciated that specific muropeptides such as the muramyl dipeptides are recognized by immune receptors in host such as NOD1, NOD2, Cryopyrin and Tolllike receptors among others (Wolf \& Underhill, 2018). The mechanism of binding and interaction of the muropeptides with these host receptors can be investigated.

III. Investigate the expression of cell-wall recycling genes in different strains.

A hallmark feature of $P$. aeruginosa is genetic adaptation found within a strain, depending on whether it causes an acute versus a chronic infection (Klockgether \& Tummler, 2017). For instance, the expression of the genes in the cell-wall recycling pathway may be different in an alginate producing strain, a phenotype found often in chronic infections. The muropeptide profile of a hyper-alginate producer PDO300 can be compared with that of non-alginate strain PAO1 (Mathee, et al., 1999). In addition, the extent of sequence conservation of the cell-wall recycling genes in clinical isolates can be discerned by in silico analyses using PAO1 as the reference strain. This can be followed up by comparing the expression of cell-wall recycling genes of these strains under different growth conditions.

IV. Changes in muropeptide profile when exposed to a different antibiotic as well as experimental analysis of muropeptide-AmpR binding.

In this study we established that the total levels of muropeptide change in the presence of an inducer such as cefoxitin which is known to be a strong inducer of AmpC $\beta$-lactamase (Jacoby, 2009). The changes in the muropeptide profile when $P$. aeruginosa is exposed to sub-lethal concentrations of $\beta$-lactams such as cefotaxime, ceftriaxone and ceftazidime which are not strong inducers of AmpC $\beta$-lactamase can 
be investigated (Jacoby, 2009). It will be interesting to note the changes in the muropeptide profile and to determine the generation of $A m p R$ under these conditions. Furthermore, it was established that GlcNAc 1, 6 anhMurNAc pentapeptide and 1, 6 anhMurNAc pentapeptide were able to bind with the AmpR effector binding domain using molecular dynamic simulation and non-denaturing mass-spectrometry (Dik, et al., 2017). An in vivo cross-linking study followed by pulldown of AmpR using specific antibodies can be also done to confirm these muropeptides as effectors. Previous study using molecular simulation dynamics focused on the important residues for effector binding in P. aeruginosa AmpR (Dik, et al., 2017). Using site-directed mutagenesis, these residues can be replaced to determine their effect on AmpC expression.

The second part of the research dealt with the role of AmpG and AmpP in $P$. aeruginosa as muropeptide transporters. This was the first attempt of looking at these transporters in an in vivo setting. However, there are still unanswered questions.

I. Role of AmpP as a muropeptide transporter can be confirmed with a transport assay.

This research elaborated on the role of the permeases AmpG and AmpP in $P$. aeruginosa. Further investigation can be done to confirm our hypothesis that AmpP acts a disaccharide-muropeptide exporter to the periplasmic space. As a previous study using fluorophore-conjugated muropeptides has shown that AmpP is unable to import disaccharide-muropeptides, in future (Perley-Robertson, et al., 2016) it can be assessed whether AmpP can export muropeptides from the cytoplasm, by monitoring the levels of fluorescence in the media, in the absence and presence of ampP expression. 
II. Role of AmpG and AmpP in antibiotic resistance and recycling can be studied in clinical-isolates of $P$. aeruginosa.

The extent of conservation of these two proteins involved in cell-wall recycling has not been studied yet. As this study primarily looked at the recycling pathway in PAO1, in future other clinical isolates and strains of $P$. aeruginosa and the role of AmpG and AmpP therein can be anlyzed

III. Role of FlgJ as an $\mathrm{N}$-acetylglucosaminidase can be confirmed through protein purification.

In the current study, we used an in-frame deletion mutant of flgJ to identify whether it was involved in producing $\mathrm{N}$-acetylglucosaminidase and antibiotic resistance. However, we were unable to obtain any clear results. A better approcach may be to purify this protein and study its activity on purified PG. The products generated in this reaction can be studied using LC/MS. This would not just confirm FlgJ as an $\mathrm{N}$ acetylglucosaminidase but would also extend to identifying if it has any other alternate enzyme activity.

IV. Besides FlgJ other putative $N$-acetylglucosaminidase can be mapped and investigated.

It may be that FlgJ is not an $N$-acetylglucosaminidase. In that case there must be an alternate enzyme that is generating the monosaccharides. One of the fulture directions will be to analyse the genome for any paralogs of FlgJ as well as NagZ. Similarities will also be chalked out according to the domains of the enzymes.

V. Analyze for an alternate transporter that shuttles monosaccharides into the periplasm.

Another possibility is that the periplasmic monosaccharides are not being generated there, but cytoplasmic ones are being transported into the periplasmic space. This 
would mean that similar to inner membrane permease AmpP which can transport disaccharides, there may be another permease which can transport monosaccharides. It may be useful to do bioinformatically analyze whether there any other inner membrane permeases that show homology with AmpG and AmpP.

The third part of the research investigated small-molecule inhibitors of an important virulence factor in $P$. aeruginosa known as $A m p R$. We were able to identify inhibitors through primary screening of compounds using our luciferase reporters. Following steps are further required to complete the investigation.

I. Identify individual family of drugs by secondary screening.

Secondary screening will be done with the individual family of drugs that were identified. Alternate promoter fusions $\mathrm{P}_{\text {phzA }}$ and $\mathrm{P}_{\operatorname{mexE}}$ will be used to confirm that inhibition of luciferase activity occurs by $A m p R$ inhibition. $A m p R$ is known to regulate phzA1 and mexE expression, positively and negatively (Balasubramanian, et al., 2012). Hence, the compounds identified so far, that decrease phzA1 expression and increase mexE expression, as inferred from luciferase activity, will be most likely AmpR inhibitors. Compounds selected post-secondary screening wherein scaffolds that inhibited AmpR activity will be further scrutinized using different functional groups.

2. Synthesize and investigate the activity of individual drugs using tertiary screening. The deconvoluted hits from the secondary screening will determine the individual compounds that will be screened on a tertiary screen. For this, individual compounds previously identified will be synthesized and their activity as luciferase inhibitors wil be checked using all three promoter systems. 
Following the identification of individual compounds and their analysis as AmpR inhibitors, they will be further analysed for properties regarding their effects on resistance and virulence. This wil include the following analysis:

MIC and synergy test: The MIC of the individual compound will be analysed by monitoring $\mathrm{OD}_{600}$ by broth microdilution analysis (EUCAST, 2000). The synergistic effect of these compounds with commonly used $P$. aeruginosa antibiotics such as $\beta$-lactams, fluroquinolones, and aminoglycosides will also be investigated suing checkerboard assays (Doern, 2014).

Cytoxicity assay: After the synthesis of the individual compounds, the cytotoxicity will be ascertained by a XTT based assay (Page, et al., 1993).

Canorhabditis elegans assay: $\quad$ C. elegans ahs been established as a god model for analysis of $P$. aeruginosa pathogenesis (Tan, et al., 1999, Balasubramanian, et al., 2012). Compounds selected so far will be tested for their effect on $C$. elegans by the fast- and slow-killing methods (Brenner, 1974).

The compounds selected will be analysed for their AmpR inhibition in clinical isolates of P. aeruginosa. This will include PA14, mucoid strain such as PA2192, Liverpool LES and PA7. 


\section{Appendix}

L-arginine regulates Pseudomonas aeruginosa pathogenesis through quorum sensing

Supurna Dhar, Katherine Vandenburg Veronica Perez, Emmanuel Duarte, Horatio Priestap, Kalai Mathee, Amir Khodamzaddeh 


\section{Abstract}

With the current widespread abuse and overuse of broad-spectrum antibiotics, resistance among bacteria is increasing at an alarming rate. Besides, the classical antibiotics that are either bacteriostatic or bactericidal, alternate targets and pathways that diminish bacterial pathogenicity are being sought after. One of these alternate approaches focusses on impeding the communication that takes place through chemical signaling between bacteria also known as quorum sensing (QS). This practice is called anti quorum-sensing. Panax ginseng is a well-established inhibitor of quorum sensing. However, previous studies were unable to decipher the active chemical compound in these extracts that was responsible for the anti-quorum sensing property. One of the compounds that were identified in ginseng extracts in high concentrations was L-arginine ( 80 mM). Further analysis with this particular amino acid on Chromobacterium violaceum showed a significant inhibition of QS regulated violacein production. In this study, L-arginine was further examined for its effects on the QS phenotypes using the opportunistic pathogen, Pseudomonas aeruginosa as a bio-monitor strain. L-arginine was found to reduce multiple QS regulated virulence factors as well as levels of Acyl Homoserine Lactone (AHL) which is one of the signaling molecules involved in QS.

Keywords: Virulence, proteases, biofilm, amino acid 


\section{Introduction}

The role of Pseudomonas aeruginosa, a major nosocomial pathogen is wellestablished in critical infections of the respiratory tracts including pan bronchiolitis, chronic obstructive pulmonary disorder, and pneumonia which are characterized by complicated treatment and often therapeutic failure (Garau \& Gomez, 2003, Valderrey, et al., 2010). This is especially seen in cystic fibrosis patients in whom chronic colonization of $P$. aeruginosa in the lungs is the result of this pathogen's inherent and acquired capacity to resist conventional antibiotic treatment (Lyczak, et al., 2000, Lyczak, et al., 2002, Jansen, et al., 2016).

With accelerated antibiotic resistance, the discovery of novel antibiotics must be prioritized (Silver, 2011). A novel approach to anti-bacterial drug discovery is to look for compounds that, without killing the bacteria maim its capacity to establish infections. As the process interrupted therein is not essential for the pathogen's survival, it does not evoke evolutionary responses from the bacteria to develop resistance, thereby increasing the long term chances of therapeutic success (Pepper, 2008, Brown, et al., 2009).

One such alternative target is the quorum sensing (QS) mechanism (O'Loughlin, et al., 2013, Eibergen, et al., 2015, Ali, et al., 2017). QS is a form of bacterial communication achieved through the production and secretion of chemical signals. $P$. aeruginosa has four systems regulating quorum sensing: Las, Rhl, Pqs, and IQS (Lee, et al., 2013). The synthases in each system produce small molecules 3-oxo-C12homoserine lactone (C12-HSL), N-butanoyl-homoserine lactone (C4-HSL), 2-heptyl-3hydroxy-4-quinolone (PQS) and 2-(2-hydroxyphenyl)-thiazole-4-carbaldehye, respectively (Papenfort \& Bassler, 2016). Conventionally, P. aeruginosa QS was known 
to exhibit hierarchy with Las system at the top of the series; however, current research suggests that regulator RhIR can affect the expression of certain target genes of the Las system (Pesci, et al., 1997, Dekimpe \& Deziel, 2009).

The signature signal is species specific and cross-communication may occur (Bassler, et al., 1997). These chemical signals allow large populations of microbes to synchronize and coordinate gene expression (Miller \& Bassler, 2001, Waters \& Bassler, 2005). It is well appreciated now that subsequent to the "quorum" formation in $P$. aeruginosa, QS can regulate an arsenal of virulence factors including extracellular as well as cell-surface associated factors thereby impacting $P$. aeruginosa host pathogenesis and clinical outcomes. A few of them include the production of secreted factors such as proteases, elastases, rhamnolipids, phenazine compounds like pyocyanin and siderophores such as pyoverdine and pyochelin (Latifi, et al., 1995, Pearson, et al., 1997, Reimmann, et al., 1997). P. aeruginosa motility such as swarming phenotype as well as biofilm formation has also been linked to quorum sensing.

Herbal medicines have always been a source of many antibiotics. In recent years however, they have been mined for their anti-QS properties (Panda, et al., 2016). Previous literature and experiments in the lab have shown that Panax ginseng, a Chinese herb does not alter $P$. aeruginosa bacterial growth but rather suppressed the production of LasA, LasB, down-regulated the synthesis of $\mathrm{AHL}$ molecules, reduced swarming motility, inhibited biofilm formation, and dispersed preformed biofilms (Song, et al., 2010, Wu, et al., 2011). Major components of Panax ginseng are the ginsenosides, among which over 30 have been discovered and extensively studied (Leung \& Wong, 2010). Experimental analyses of the ginsenosides exhibited no alteration of quorumsensing phenotypes (Song, et al., 2010). 
Aside from the ginsenosides, aqueous extracts of $P$. ginseng contain many other non-nitrogen and nitrogen-based compounds including organic acids, peptides, amines, nucleic acids and free amino acids (FAA)(Attele, et al., 1999). Studies have shown that the FAA content of $P$. ginseng constitutes about $45 \%$ of the total amino acids and that arginine was responsible for about $60 \%$ of the total FAA (Kwon, et al., 1990, Park, et al., 1990). In this study, we hypothesize that the high content of free arginine in $P$. ginseng contributes to the anti-QS effects seen in its aqueous extracts. To investigate this, the effects of L-arginine on the quorum sensing phenotypes were analyzed.

\section{MATERIALS AND METHODS}

Bacterial strains, growth media and culture conditions. Pseudomonas aeruginosa PAO1 (Holloway \& Morgan, 1986), PAO $\Delta r e t S$, PAO $\Delta a l g D \Delta p e l \Delta p s l$, were used for analysis of QS phenotypes. Staphylococcus aureus (ATCC 12600) was used for the assay of LasA protease (Kessler, et al., 1993). All bacteria were maintained on Luria broth (LB) and grown overnight at $37^{\circ} \mathrm{C}$. L-arginine (Sigma-Aldrich) dissolved in LB for the assays.

Growth curves. The effect of L-arginine on PAO1 growth was determined by monitoring the O.D.600 for 16 hours. Briefly, an overnight culture of PAO1 was spun down. The cells were washed with $0.85 \%$ saline to remove spent media and diluted to O.D. 1 in fresh LB. From this, the culture was diluted further to an O.D. of 0.025 in fresh LB media in 48-well plates (Falcon) for monitoring growth. L-arginine at the desired concentrations was added and the absorbance at $600 \mathrm{~nm}$ was monitored at one-hour intervals using BioTek Synergy HT (Winooski, VT, USA) plate reader for 17 hours at $37{ }^{\circ} \mathrm{C}$. 
Swarming assay. The assay was performed on plates containing $0.65 \%$ agar with Larginine at the test concentrations. An overnight culture of PAO1 was diluted to an $\mathrm{OD}_{600}$ of 1 . One $\mu \mathrm{L}$ of this culture was spotted onto autoclaved-sterile discs. The plates were placed in a $30^{\circ} \mathrm{C}$ incubator and the swarming motility was measured every 24 and 48 hours (Henrichsen, 1972, Kohler, et al., 2000).

LasA staphylolytic assay. The LasA protease activity was determined as previously described (Kessler, et al., 1993, Kong, et al., 2005). Briefly, P. aeruginosa was grown overnight with and without L-arginine. A $20 \mu \mathrm{L}$ of the culture supernatant was added to $180 \mu \mathrm{L}$ of boiled $S$. aureus. The lysis of $S$. aureus was monitored every 10 minutes by recording the $\mathrm{OD}_{600}$ at $37{ }^{\circ} \mathrm{C}$ for an hour. The activity was noted as the decrease in $\mathrm{OD}_{600}$ per hour.

LasB elastolytic assay. Elastin Congo red (ECR, Sigma, St. Louis, MO) was used to determine the role of L-arginine in regulating the elastolytic activity of $P$. aeruginosa PAO1 as described before (Ohman, et al., 1980). Briefly, $900 \mu \mathrm{l}$ of ECR buffer containing $100 \mathrm{mM}$ Tris, $1 \mathrm{mM} \mathrm{Cacl}$, $\mathrm{pH}$ 7.6-7.5 was made with $20 \mathrm{mg}$ of ECR and vortexed to dissolve. To this, a $100 \mu \mathrm{l}$ aliquot of $P$. aeruginosa supernatants was added. This mixture was incubated with shaking at $37{ }^{\circ} \mathrm{C}$ for one hour. Insoluble ECR was removed by centrifugation. Activity was expressed as the change in $\mathrm{OD}_{495}$ after one-hour incubation (Nishino \& Powers, 1980, Ohman, et al., 1980). 
Pyocyanin assay. Overnight cultures of $P$. aeruginosa PAO1 with L-arginine was grown for 18-20 hours to obtain bacteria at stationary phase. The optical densities of all bacteria were normalized before the assay. $5 \mathrm{ml}$ of the supernatant was extracted into a tube containing $3 \mathrm{ml}$ of chloroform and vortexed. This was centrifuged, and the top layer removed. To the bottom layer, $1 \mathrm{ml}$ of $0.2 \mathrm{~N} \mathrm{HCl}$ was added. This was vortexed and centrifuged. The top (pink) aqueous phase was removed into a cuvette and the absorbance at $520 \mathrm{~nm}$ recorded. The absorbance is multiplied by 17.072 indicating the concentration of pyocyanin produced per $\mathrm{ml}$ of culture $(\mu \mathrm{g} / \mathrm{ml})$ (Kurachi, 1958, Essar, et al., 1990).

Biofilm assay. Biofilm assay was performed in tubes as described previously (Friedman \& Kolter, 2004, O'Toole, 2011). Briefly, overnight cultures of PAO1 grown on LB agar plates were resuspended in LB to a final dilution of 0.0025 . The test samples contained L-arginine at 25 and $50 \mathrm{mM}$. PAO $\Delta$ retS and PAO $\Delta$ algD $\Delta$ pel $\Delta p s /$ were kept as positive and negative controls, respectively (Goodman, et al., 2004). All the tubes were incubated at $37^{\circ} \mathrm{C}$ for $24-48$ hours. Subsequently, the culture was discarded and the tubes washed in bins containing water and left at room temperature for 10 minutes. This was stained with $0.1 \%$ crystal violet. The dye was removed after 20 minutes and the tubes were washed thoroughly with running tap-water. The attached dye was then solubilized with absolute ethanol and biofilm formation was quantified by recording the absorbance at $590 \mathrm{~nm}$.

AHL assay by GC/MS. AHL extraction from PAO1 supernatants was done using previously described protocols (Makemson, et al., 2006). P. aeruginosa cultures were 
grown for 18-20 hrs and their OD normalized. The cells were spun down and the supernatant was removed in a separate tube and prepared for AHL detection by gas chromatography/mass spectrometry (Cataldi, et al., 2004, Kusar, et al., 2016). Briefly, the supernatant is divided in equal volumes into two separate tubes. One was treated with $20 \mu \mathrm{L} / \mathrm{mL}$ acetic acid and the other with $10 \mu \mathrm{L} / \mathrm{mL}$ of $8 \mathrm{~N} \mathrm{NaOH}$. These samples were both further extracted three times with equal parts of acidified ethyl acetate on a rotating platform at room temperature. The acidic and basic extracts were dehydrated at $35{ }^{\circ} \mathrm{C}$ until only a pellet is left. These are resuspended in DMSO and concentrated 20-fold before analyses. For mass-spectrometry controls, synthetic samples of $P$. aeruginosa $\mathrm{N}$-butanoyl AHL and N-dodecanoyl AHL (Sigma-Aldrich) at a concentration of 500 ppm were subjected to the same extraction. The analyses were done by GC-QTOF. The instruments used were Agilent GC coupled to a Q-TOF, with a HP-5MS-UI column (30 m $\times 0.25 \mathrm{~mm} \times 0.25 \mu \mathrm{m})$. Data was acquired over $9.83 \mathrm{~min}$ with an initial temperature of $150{ }^{\circ} \mathrm{C}$ held for $0.5 \mathrm{~min}$, with an increase to a final temperature of $275{ }^{\circ} \mathrm{C}$, at $15{ }^{\circ} \mathrm{C}$ per minute.

\section{RESULTS}

L-arginine shows dose-dependent effect on growth. The growth of PAO1 without Larginine was compared to the growth with $25,30,35,40,45$, and $50 \mathrm{mM}$ of L-arginine in the media. On monitoring growth of PAO1 over 15-17 hours, a decreased growth rate was observed with 45 and $50 \mathrm{mM}$ of arginine, during the log phase. $P$. ginseng contains very high concentration of L-arginine $(80 \mathrm{Mm})$ and our preliminary experiments indicated that anti-QS effects were noticed specifically a higher concentration of L-arginine. Henceforth, experiments were conducted in the presence of two concentrations of L- 
arginine, 25 and $50 \mathrm{mM}$. However, all experiments were conducted after cultures reached stationary phase with normalization of OD 600 of all cultures before assay.
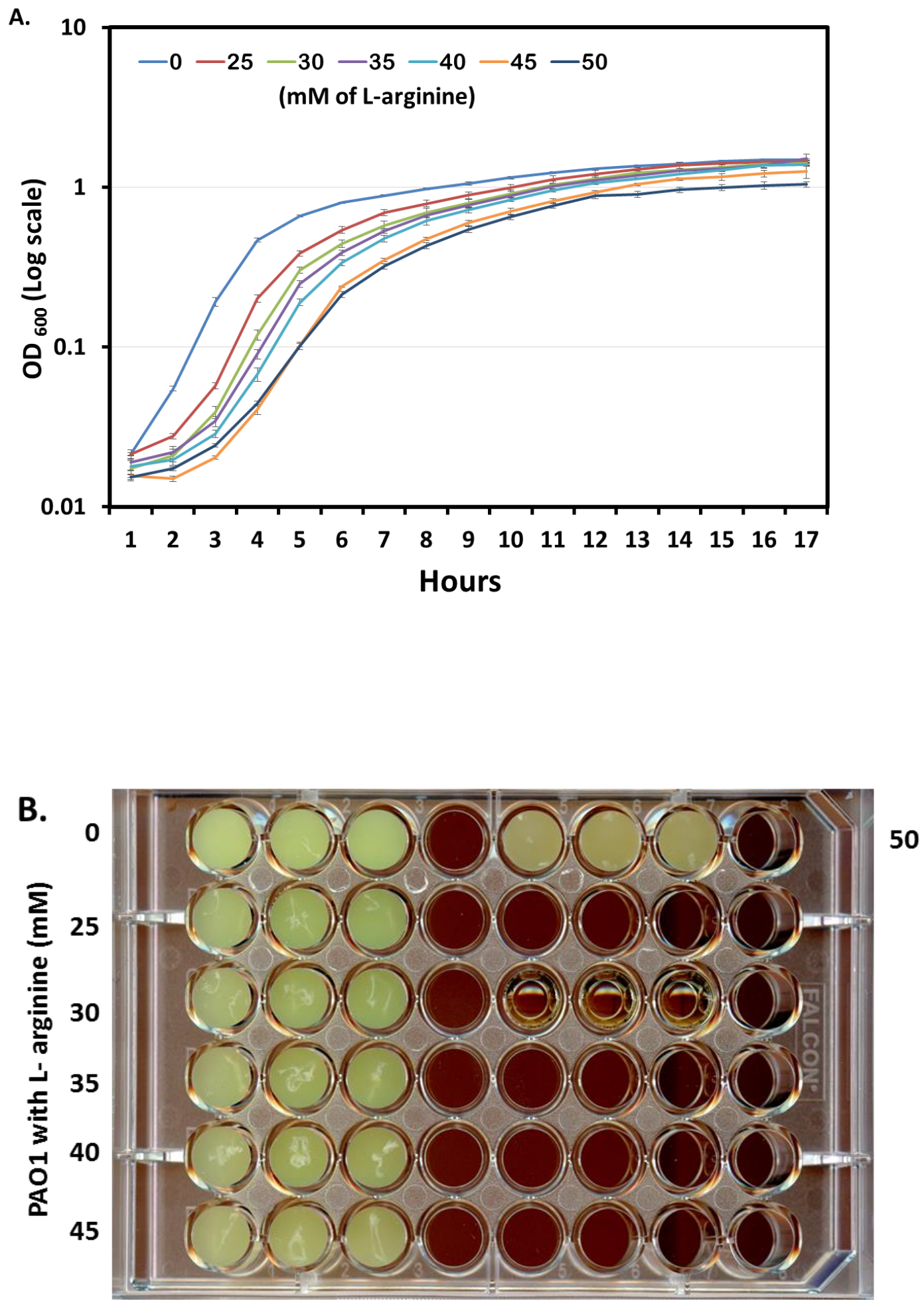
Figure 1. Growth of PAO1 in presence of L- arginine. A. The growth of PAO1 at 0 , $25,30,35,40,45$ and $50 \mathrm{mM}$ of L-arginine was monitored over $15-17$ hours at $37{ }^{\circ} \mathrm{C}$. There was a significant difference in growth between PAOI (0mM) and PAO1 (45 and $50 \mathrm{mM})$ during log-phase, as determined by unpaired t-test $(p<0.05)$. B. The image of the plate after 17 hours of growth is shown. The PAO1 cultures grown with 0,25 and 50 $\mathrm{mM}$ were chosen for further analysis of QS phenotypes. All the cultures were normalized to the same O.D. before continuing the assays.

L-arginine reduces production of LasA protease. $P$. aeruginosa is known to produce a host of secreted virulence factors which includes proteases that are instrumental in establishing acute infections (Schad \& Iglewski, 1988). One of these is LasA which is a staphylolytic metalloendopeptidase whose regulation is controlled by QS (Ohman, et al., 1980, Kessler, 1995). Expression of LasA can be analyzed spectrophotometrically by monitoring the change in $\mathrm{OD}_{600}$ due to lysis of $S$. aureus (Kessler, et al., 1993, Engel, et al., 1998). The LasA protease activity was quantified in our control which contained supernatant from PAO1 cultures and compared with our testing solutions which had supernatants from PAOI grown with 25 and $50 \mathrm{mM}$ of L-arginine. Supernatants from PAO1 exposed to L-arginine both at the lower $25 \mathrm{mM}$ and the higher concentration of 50 $\mathrm{mM}$ showed a dose-dependent decrease in production of this protease, which was not statistically significant (Figure 2).

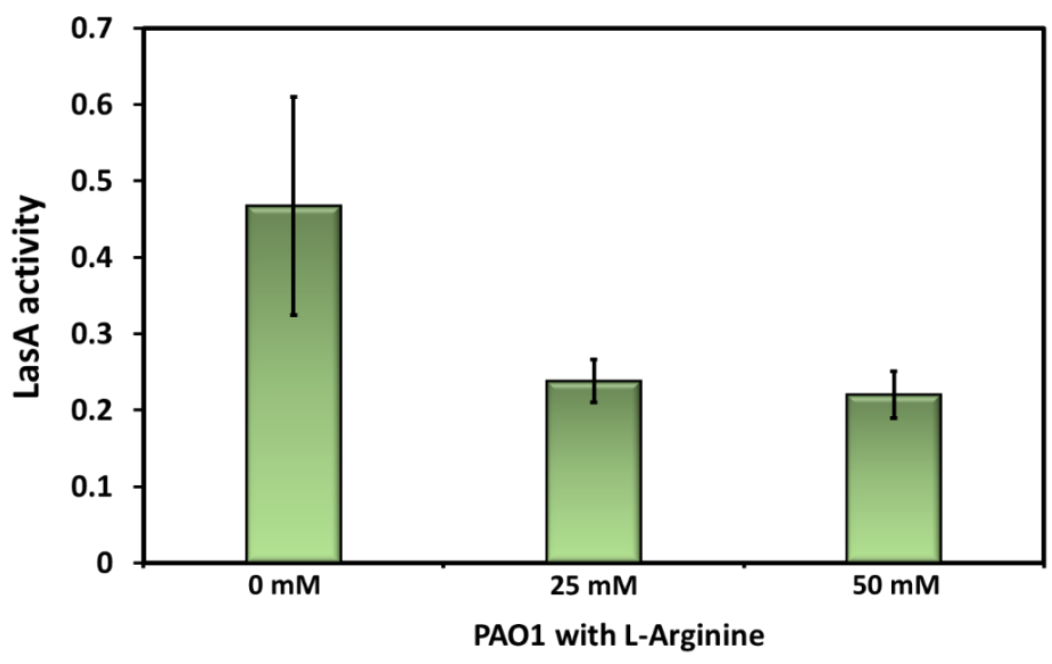


Figure 2. Las A activity in PAO1 supernatants in presence of L-arginine. Las A was quantified as the change in $\mathrm{OD}_{600}$ over one hour at $37^{\circ} \mathrm{C}$ per $\mu \mathrm{g}$ of protein. The levels of LasA produced by PAO1 in presence of L-arginine were reduced. However, the decrease was not statistically significant $(p>0.05)$ as determined unpaired t-test.

L-arginine reduces production of LasB elastase. The $P$. aeruginosa LasB elastase is also under the control of QS. This protease degrades elastin which is measured using the Elastin Congo Red assay (Ohman, et al., 1980). Under normal circumstances, the supernatant of a $P$. aeruginosa culture degrades elastin leading to an increase in $\mathrm{OD}_{495}$. It was found that the production of elastin by $P$. aeruginosa was significantly reduced at the higher concentration of L-arginine of $50 \mathrm{mM}$, when compared to the supernatant extracted from the strain PAO1 grown without any L-arginine (Figure 3).

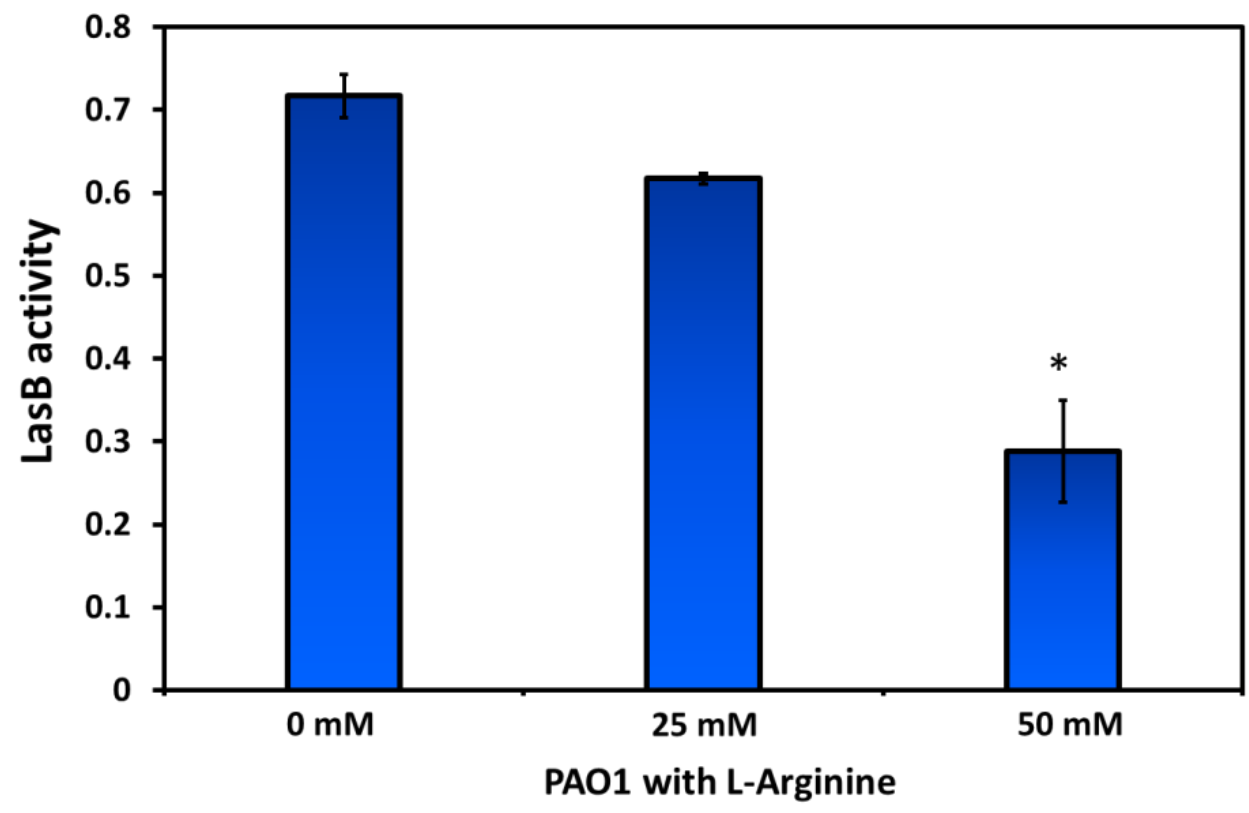

Figure 3. LasB activity in PAO1 supernatants in presence of L-arginine. Elastase activity is expressed as the absorbance at $\mathrm{OD}_{495}$ per microgram of protein in PAO1 supernatants. The levels of elastase were decreased significantly in PAO1 with $50 \mathrm{mM}$ L-arginine, $p<0.05$ as determined by unpaired T-test. 
L-arginine reduces pyocyanin formation. To further investigate if L-arginine affects the QS-dependent production of pyocyanin. Pyocyanin is one of the redox blue-green pigments produced by Pseudomonads that is frequently associated with acute and chronic infections (Caldwell, et al., 2009). Compared to PAO1 cultured without Larginine, the pyocyanin produced in the presence of L-arginine was significantly reduced. (Figure 4).

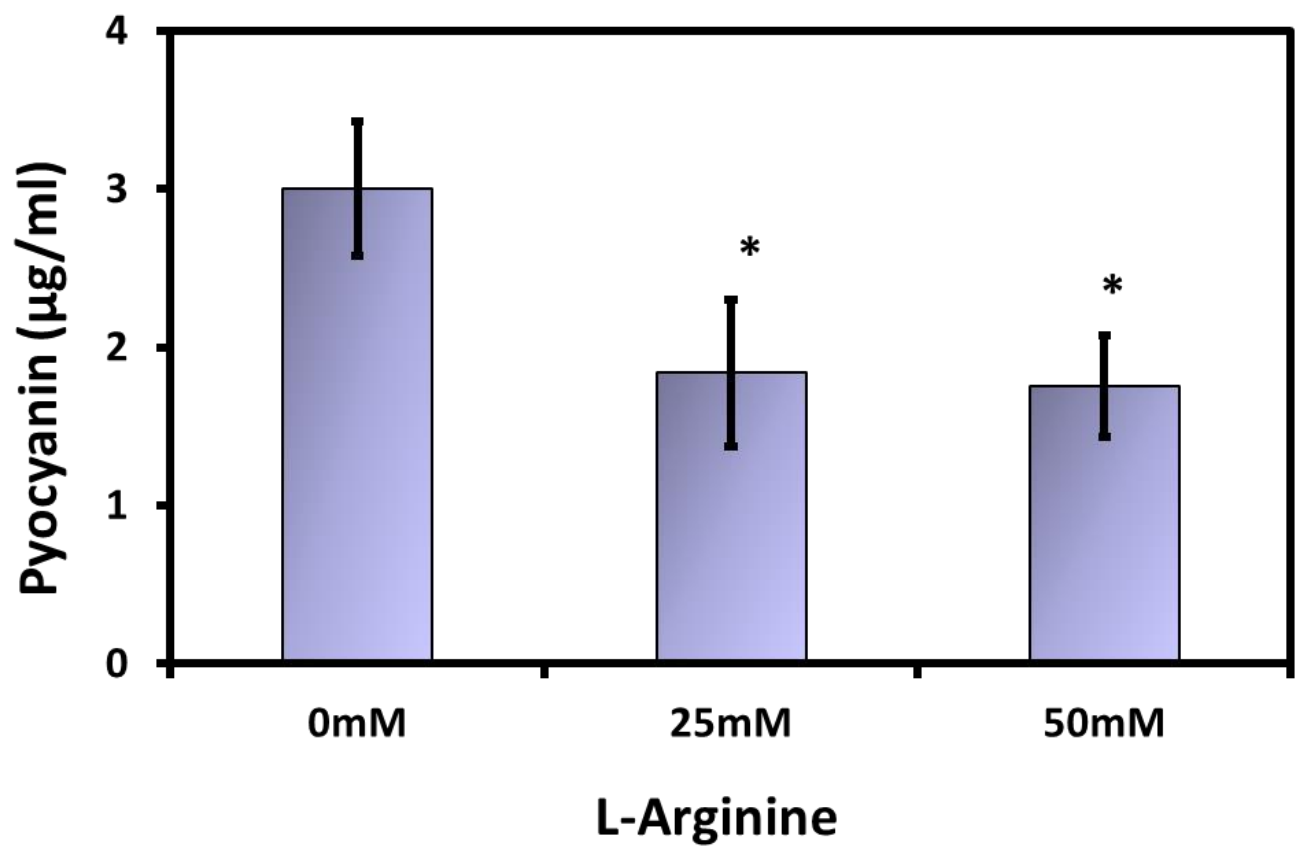

Figure 4. Effect of L-arginine on pyocyanin production. Increasing concentrations of L-arginine led to a decrease in pyocyanin production in PAO1. This decrease was statistically significant at both 25 and $50 \mathrm{mM}\left({ }^{*} p<0.05,0 \mathrm{mM}\right.$ vs $25 \mathrm{mM}, 0 \mathrm{mM}$ vs $50 \mathrm{mM}$, as determined by unpaired T- test). 
L-arginine reduces biofilm formation. Biofilm formation is a chronic-infection phenotype that is in-part regulated by QS (Davies, et al., 1998). Positive control $\mathrm{PAO} \Delta$ retS showed a hyper-biofilm formation whereas negative control $\mathrm{PAO} \Delta$ algD $\Delta p e l \Delta p s /$ showed negligible biofilm as quantified by crystal violet (Figure 5). Presence of L-arginine resulted in a decrease in biofilm formation in a dose-dependent manner.
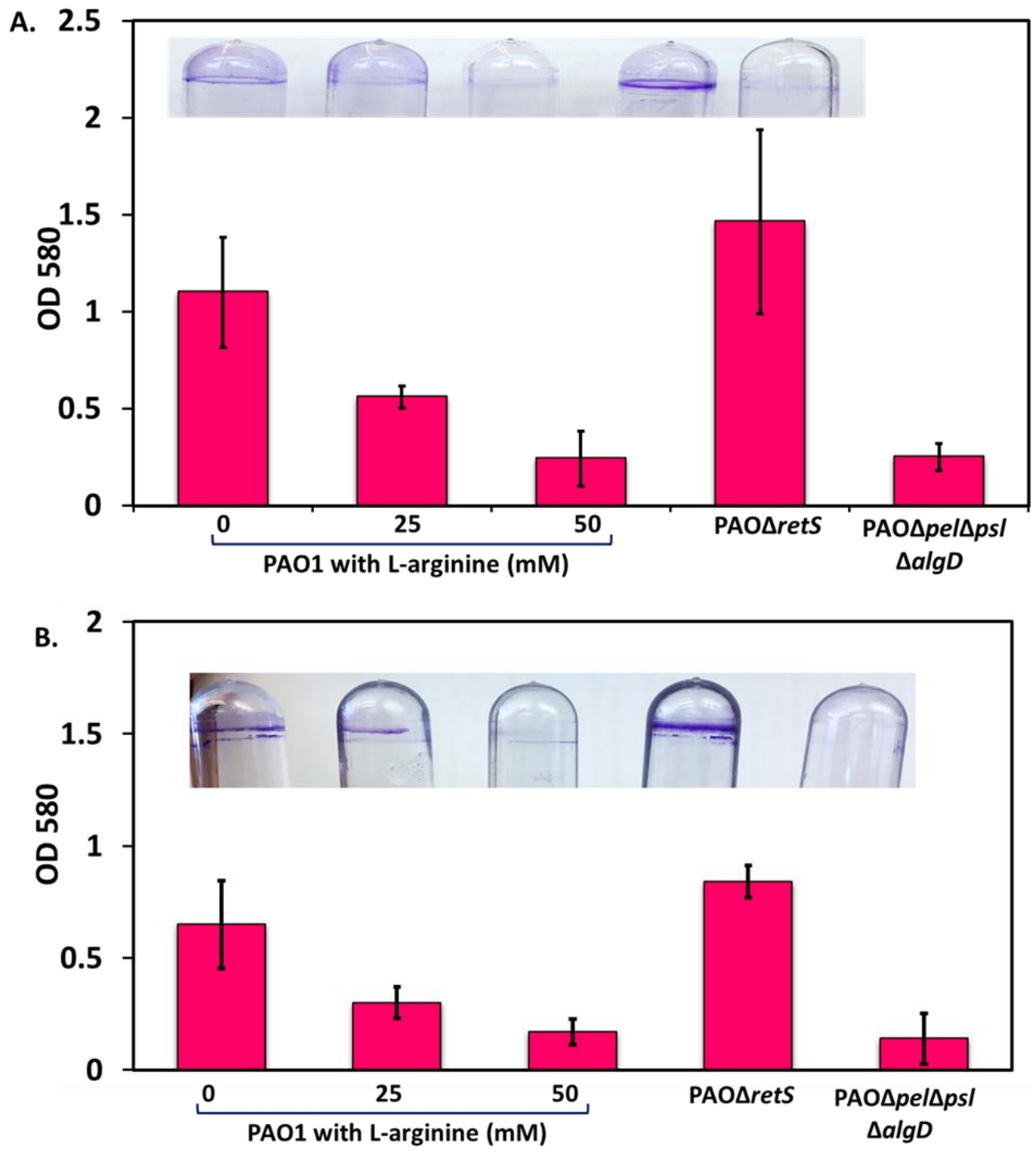
Figure 5. Effect of arginine on biofilm formation. The strains tested were PAO1 in increasing concentrations of L-arginine. As positive control of the assay PAO $\Delta$ retS which is a biofilm hyperproducer and PAO $\Delta$ algD $\Delta$ pel $\Delta \mathrm{psl}$ a non-biofilm producer were also assayed. A. After 24 hours, a significant difference $\left({ }^{*} p<0.05\right)$ was observed between PAO1 and PAO1 cultured with 25 and $50 \mathrm{mM}$ of L-arginine, as determined by unpaired t-test. B. After 48 hours, a significant difference $\left({ }^{*} p<0.05\right)$ was found between PAO1 with $0 \mathrm{mM}$ of L-arginine and $50 \mathrm{mM}$ of L-arginine, as determined by unpaired $\mathrm{t}$ test.

\section{L-arginine reduces production of acyl-homoserine lactone (AHL). Direct} identification of $\mathrm{AHL}$ was achieved from supernatants by gas-chromatography/massspectrometry. For extraction of AHLs, PAO1 supernatants were treated with acid and base, according to previously established protocols (Makemson, et al., 2006). The acid stabilizes the lactone ring whereas the base leads to opening of the lactone ring and destabilizes the signaling molecules. As positive controls for the assay, N-butyryl-LHomoserine lactone (C4-AHL) and N-(3-Oxododecanoyl)-L-homoserine lactone (C12$\mathrm{AHL}$ ) were dissolved in LB and extracted using the same procedure as the test samples. The presence of signaling molecules was also analyzed in LB media, and the supernatants extracted from PAO1 culture with and without L-arginine. It was found that cultures grown with L-arginine at $50 \mathrm{mM}$ reduced the $\mathrm{AHL}$ production as seen from the decreased peak intensity (Figure 6). 


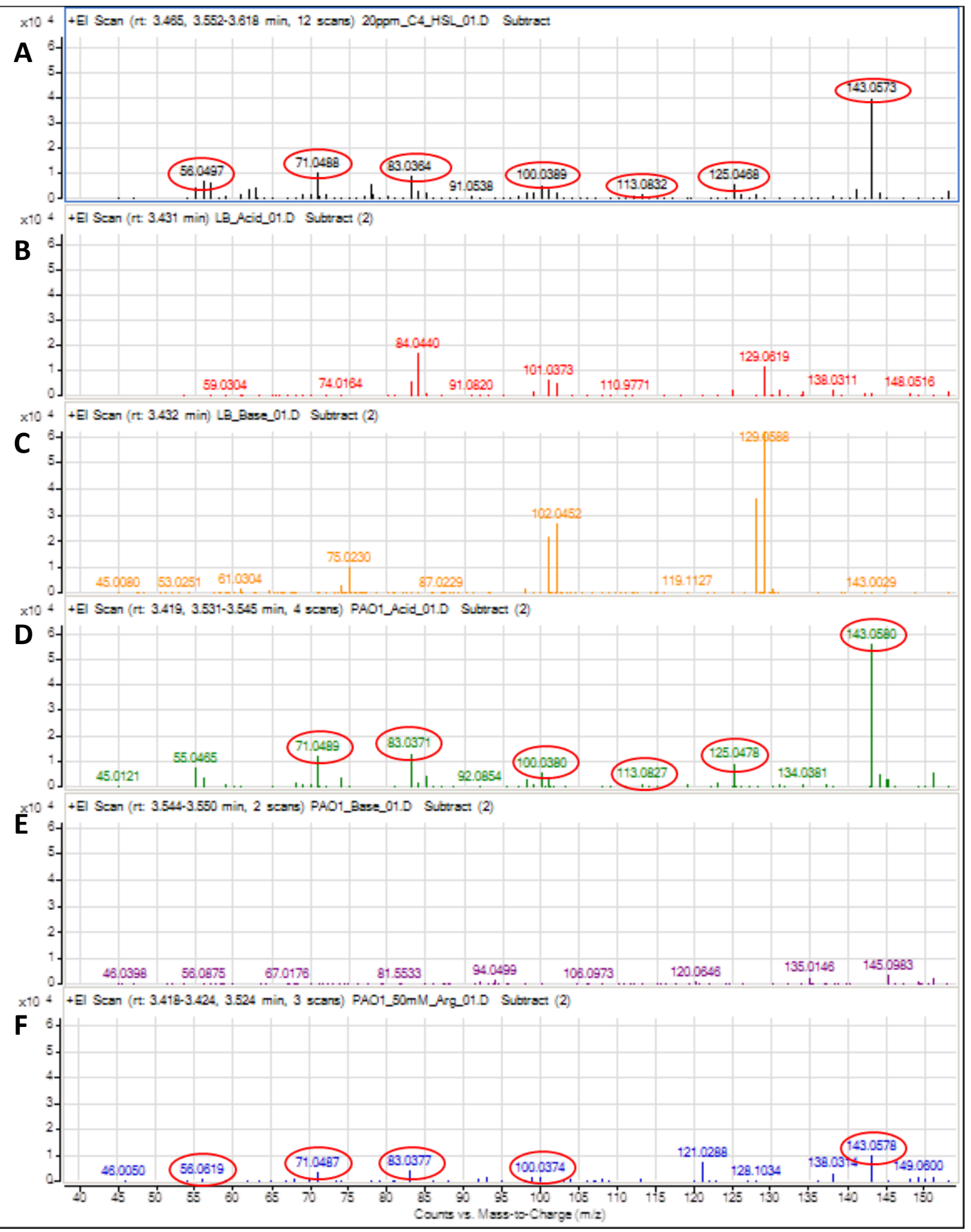


Figure 6. A. Panel shows the representative fragmentation spectra of an acylhomoserine lactone. B and C. Panel shows the media control for the experimental samples. LB treated with acid and bases are analyzed for the representative ions. $\mathbf{D}$. Supernatant from PAO1 growth culture without arginine in the growth media was treated with acid and examined for fragmentation spectra. The circled ions were found in the culture supernatant indicative of AHL. E. Panel shows the same supernatant extracted from PAO1 and treated with base. The alkaline treatment breaks the lactone ring and destabilizes the AHLs. Here this is confirmed as we don't observe any of the ions from the spectra. F. Panel shows the supernatant extracted from PAO1 cultured with $50 \mathrm{mM}$ L-arginine. As can be observed, the levels of ions were far lower; especially the most abundant ion with $\mathrm{m} / \mathrm{z} 143$ was detected at reduced levels.

\section{DISCUSSION}

Given the abundance of research that illustrates QS as a critical element of bacterial pathogenesis, it is but natural that identifying anti-QS compounds as alternative/synergistic therapies will be advantageous.

Previous studies in our lab examined herbs with known medicinal properties (Adonizio, et al., 2008, Song, et al., 2010). One of these, Panax ginseng, has known immunomodulatory and anti-QS properties (Larsen, et al., 2004, Song, et al., 2010, Wu, et al., 2011, Schneper L., 2012). Experiments with ginsenosides purified from $P$. ginseng did not account for the anti-QS effects (Song, et al., 2010). Besides ginsenosides, $P$. ginseng contains many other bioactive compounds one of which is free amino-acids (Hou, 1977). In this study, the ability of arginine to alter QS was discerned by measuring QS-dependent production of secreted virulence factors such as protease LasA, elastase LasB, and pyocyanin of $P$. aeruginosa. Furthermore, we also determined the effect of arginine on phenotypes critical for host-colonization and sustained infection such as motility and biofilm formation. 
Arginine affects the production of QS-regulated virulence factors. The extracellular virulence factors are significant for causing acute infection succeeding colonization. Production of alkaline LasA protease and elastase LasB is determined by the presence of both C-12 and C-4 HSL secreted respectively by the Las and Rhl QS systems (Gambello \& Iglewski, 1991, Gambello, et al., 1993, Reimmann, et al., 1997). LasB elastase has immunomodulatory properties and is essential to cleave any elastin containing tissue in a host such as the human lung and is one of the chief components permitting invasive infections (Heck, et al., 1990, Galloway, 1991). LasA is a metalloendopeptidase which has been implicated as an important virulence factor particularly in corneal infections (Preston, et al., 1997). Along with these proteases, the presence of Larginine also resulted in decreased production of pyocyanin. Pyocyanin is a blue-green phenazine pigment which is a redox-active compound and facilitates the formation of reactive oxygen stress resulting in tissue injury (Mavrodi, et al., 2001). Previously, it has been shown that extracts of $5 \%$ ginseng cause a significant reduction in protease and elastase production. There was, however, a dose-dependent effect wherein the lower concentration of ginseng appeared to increase elastase activity slightly (Song, et al., 2010). In our results, we also witnessed a dose-dependent effect of L-arginine supplementation with increased anti-QS activity observed at higher concentrations (Figure 2, 3, and 5). This decrease in production of virulence was correlated with a decrease in production of HSL molecules as determined by GC/MS in the presence of 50mM L-arginine (Figure 7).

Arginine affects biofilm formation. Previous studies with $P$. ginseng have shown reduced swarming motility and biofilm formation in $P$. aeruginosa PAO1. In our study, we examined the formation of biofilm upto 48 hours. Biofilms are an organized structure 
composed of polysaccharides (Pel, Psl, and alginate), extracellular DNA and proteins that encase the bacterial community thereby protecting it from certain host-defenses and antibiotics (Flemming \& Wingender, 2010). Biofilm development is a multi-factorial process that depends on swarming and twitching motility and QS (production of C12HSL by Lasl synthase) (O'Toole \& Kolter, 1998, Patriquin, et al., 2008). In our study, we saw a progressive decrease of biofilm formation in the presence of increasing concentrations of L-arginine (Figure 5). This decrease could be due to the inhibition of $\mathrm{AHL}$ production/transport as well as to the reduced swarming motility that is observed with L-arginine at these concentrations. This is an interesting observation as previous studies done with PA14 which employed a much lower concentration of L-arginine $(4.8 \mathrm{mM})$ showed a robust increase in biofilm formation with a reduction in swarming motility owing to modulation of the secondary metabolite c-di-GMP by L-arginine (Bernier, et al., 2011).

In mice burn-wound model, arginine has shown to prevent bacterial dissemination and sepsis (Everett, et al., 2017). Depletion of arginine in burn-wound was associated with increased arginase. Supplementation with L-arginine at concentrations deemed safe for use in humans, post $P$. aeruginosa infection, resulted in the decreased bacterial spread and prolonged animal survival (Everett, et al., 2017). This suggests that arginine may be a valuable alternate therapy for prevention of sepsis in burn-wound patients. The physiologic role of arginine in the human body is well-documented and some mirror the effects of $P$. ginseng, including stimulating the immune system and exhibiting anti-cancer properties (Tong \& Barbul, 2004). Consuming L-arginine in doses larger than normal dietary intake has also been linked to improved endothelial dysfunction and overall vascular health (Kamada, et al., 2001). As an anti-pseudomonal agent, L-arginine has 
shown increased biofilm susceptibility to antibiotics both within mature biofilms and in aerobic/anaerobic conditions (Borriello, et al., 2006). Studies on the effects of inhaled buffered L-arginine in the treatment of CF has shown a significant improvement in thoracic gas volume, arterial $\mathrm{PO}_{2}$ and specific conductance (Solomons, et al., 1971). Our results also highlight the probability that $L$-arginine by reducing bacterial virulence may be a beneficial additive or alternate component of combination therapy in $P$. aeruginosa infections. 


\section{References}

Etymologia: Pseudomonas Emerging Infectious Diseases. 18.

Abraham EP \& Chain E (1988) An enzyme from bacteria able to destroy penicillin. 1940. Reviews of infectious diseases 10: 677-678.

Acebron I, Mahasenan KV, De Benedetti S, et al. (2017) Catalytic cycle of the NAcetylglucosaminidase NagZ from Pseudomonas aeruginosa. Journal of the American Chemical Society 139: 6795-6798.

Adin DM, Engle JT, Goldman WE, McFall-Ngai MJ \& Stabb EV (2009) Mutations in ampG and lytic transglycosylase genes affect the net release of peptidoglycan monomers from Vibrio fischeri. Journal of bacteriology 191: 2012-2022.

Adonizio A, Kong KF \& Mathee K (2008) Inhibition of quorum sensing-controlled virulence factor production in Pseudomonas aeruginosa by South Florida plant extracts. Antimicrobial agents and chemotherapy 52: 198-203.

Aendekerk S, Ghysels B, Cornelis P \& Baysse C (2002) Characterization of a new efflux pump, MexGHI-OpmD, from Pseudomonas aeruginosa that confers resistance to vanadium. Microbiology 148: 2371-2381.

Aizawa SI (1996) Flagellar assembly in Salmonella typhimurium. Molecular microbiology 19: 1-5.

al-Bar OA, O'Connor CD, Giles IG \& Akhtar M (1992) D-alanine: D-alanine ligase of Escherichia coli. Expression, purification and inhibitory studies on the cloned enzyme. The Biochemical journal 282 ( Pt 3): 747-752.

Alhede M, Bjarnsholt T, Jensen PO, et al. (2009) Pseudomonas aeruginosa recognizes and responds aggressively to the presence of polymorphonuclear leukocytes. Microbiology 155: 3500-3508.

Ali L, Goraya MU, Arafat Y, Ajmal M, Chen JL \& Yu D (2017) Molecular mechanism of quorum-sensing in Enterococcus faecalis: Its role in virulence and therapeutic approaches. International journal of molecular sciences 18.

Allison C \& Hughes C (1991) Bacterial swarming: an example of prokaryotic differentiation and multicellular behaviour. Science progress 75: 403-422.

Amanuma H \& Strominger JL (1980) Purification and properties of penicillin-binding proteins 5 and 6 from Escherichia coli membranes. The Journal of biological chemistry 255: $11173-11180$.

Ambler RP (1980) The structure of beta-lactamases. Philosophical transactions of the Royal Society of London. Series B, Biological sciences 289: 321-331.

Amsler K, Santoro C, Foleno B, Bush K \& Flamm R (2010) Comparison of broth microdilution, agar dilution, and Etest for susceptibility testing of doripenem against Gram-negative and Gram-positive pathogens. Journal of clinical microbiology 48: 33533357.

Anderson JS, Matsuhashi M, Haskin MA \& Strominger JL (1965) Lipidphosphoacetylmuramyl-pentapeptide and lipid-phosphodisaccharide-pentapeptide: 
presumed membrane transport intermediates in cell wall synthesis. Proceedings of the National Academy of Sciences of the United States of America 53: 881-889.

Angus BL, Carey AM, Caron DA, Kropinski AM \& Hancock RE (1982) Outer membrane permeability in Pseudomonas aeruginosa: comparison of a wild-type with an antibioticsupersusceptible mutant. Antimicrobial agents and chemotherapy 21: 299-309.

Apel D \& Surette MG (2008) Bringing order to a complex molecular machine: the assembly of the bacterial flagella. Biochim Biophys Acta 1778: 1851-1858.

Arora SK, Ritchings BW, Almira EC, Lory S \& Ramphal R (1997) A transcriptional activator, FleQ, regulates mucin adhesion and flagellar gene expression in Pseudomonas aeruginosa in a cascade manner. J Bacteriol 179: 5574-5581.

Asgarali A, Stubbs KA, Oliver A, Vocadlo DJ \& Mark BL (2009) Inactivation of the glycoside hydrolase NagZ attenuates antipseudomonal beta-lactam resistance in Pseudomonas aeruginosa. Antimicrobial agents and chemotherapy 53: 2274-2282.

Asoh S, Matsuzawa H, Ishino F, Strominger JL, Matsuhashi M \& Ohta T (1986) Nucleotide sequence of the $p b p A$ gene and characteristics of the deduced amino acid sequence of penicillin-binding protein 2 of Escherichia coli K12. European journal of biochemistry 160: 231-238.

Attele AS, Wu JA \& Yuan CS (1999) Ginseng pharmacology: multiple constituents and multiple actions. Biochemical pharmacology 58: 1685-1693.

Avison MB, Horton RE, Walsh TR \& Bennett PM (2001) Escherichia coli CreBC is a global regulator of gene expression that responds to growth in minimal media. The Journal of biological chemistry 276: 26955-26961.

Avison MB, Niumsup P, Nurmahomed K, Walsh TR \& Bennett PM (2004) Role of the 'cre/blr-tag' DNA sequence in regulation of gene expression by the Aeromonas hydrophila beta-lactamase regulator, BlrA. The Journal of antimicrobial chemotherapy 53: 197-202.

Azucena E \& Mobashery S (2001) Aminoglycoside-modifying enzymes: mechanisms of catalytic processes and inhibition. Drug resistance updates : reviews and commentaries in antimicrobial and anticancer chemotherapy 4: 106-117.

Azzolina B \& El-Sherbeini M (2002) Mray gene and enzyme of Pseudomonas aeruginosa. ed.^eds.), p.^pp. Google Patents.

Azzolina BA, Yuan X, Anderson MS \& El-Sherbeini M (2001) The cell wall and cell division gene cluster in the Mra operon of Pseudomonas aeruginosa: cloning, production, and purification of active enzymes. Protein expression and purification 21: 393-400.

Babouee Flury B, Ellington MJ, Hopkins KL, et al. (2016) Association of novel nonsynonymous single nucleotide polymorphisms in $a m p D$ with cephalosporin resistance and phylogenetic variations in $a m p C$, $a m p R$, ompF, and ompC in Enterobacter cloacae isolates that are highly resistant to carbapenems. Antimicrobial agents and chemotherapy 60: 2383-2390.

Bacik JP, Whitworth GE, Stubbs KA, et al. (2011) Molecular basis of 1,6-anhydro bond cleavage and phosphoryl transfer by Pseudomonas aeruginosa 1,6-anhydro-Nacetylmuramic acid kinase. The Journal of biological chemistry 286: 12283-12291. 
Backhed F, Normark S, Schweda EK, Oscarson S \& Richter-Dahlfors A (2003) Structural requirements for TLR4-mediated LPS signalling: a biological role for LPS modifications. Microbes and infection 5: 1057-1063.

Bader MS, Loeb M \& Brooks AA (2017) An update on the management of urinary tract infections in the era of antimicrobial resistance. Postgraduate medicine 129: 242-258.

Badet B, Vermoote P, Haumont PY, Lederer F \& LeGoffic F (1987) Glucosamine synthetase from Escherichia coli: purification, properties, and glutamine-utilizing site location. Biochemistry 26: 1940-1948.

Balasubramanian D, Kumari H \& Mathee K (2015) Pseudomonas aeruginosa AmpR: an acute-chronic switch regulator. Pathogens and disease 73: 1-14.

Balasubramanian D, Schneper L, Kumari H \& Mathee K (2013) A dynamic and intricate regulatory network determines Pseudomonas aeruginosa virulence. Nucleic acids research 41: 1-20.

Balasubramanian D, Schneper L, Merighi M, Smith R, Narasimhan G, Lory S \& Mathee K (2012) The regulatory repertoire of Pseudomonas aeruginosa AmpC beta-lactamase regulator AmpR includes virulence genes. PloS one 7: e34067.

Balasubramanian D, Kumari $H$, Jaric $M$, et al. (2014) Deep sequencing analyses expands the Pseudomonas aeruginosa AmpR regulon to include small RNA-mediated regulation of iron acquisition, heat shock and oxidative stress response. Nucleic acids research 42: 979-998.

Balcewich MD, Stubbs KA, He Y, James TW, Davies GJ, Vocadlo DJ \& Mark BL (2009) Insight into a strategy for attenuating AmpC-mediated beta-lactam resistance: structural basis for selective inhibition of the glycoside hydrolase NagZ. Protein science : a publication of the Protein Society 18: 1541-1551.

Baquero MR, Bouzon M, Quintela JC, Ayala JA \& Moreno F (1996) dacD, an Escherichia coli gene encoding a novel penicillin-binding protein (PBP6b) with DDcarboxypeptidase activity. Journal of bacteriology 178: 7106-7111.

Barreteau H, Kovac A, Boniface A, Sova M, Gobec S \& Blanot D (2008) Cytoplasmic steps of peptidoglycan biosynthesis. FEMS microbiology reviews 32: 168-207.

Bartowsky E \& Normark S (1993) Interactions of wild-type and mutant AmpR of Citrobacter freundii with target DNA. Molecular microbiology 10: 555-565.

Bassler BL, Greenberg EP \& Stevens AM (1997) Cross-species induction of luminescence in the quorum-sensing bacterium Vibrio harveyi. Journal of bacteriology 179: 4043-4045.

Bauernfeind A, Stemplinger I, Jungwirth R \& Giamarellou H (1996) Characterization of the plasmidic beta-lactamase CMY-2, which is responsible for cephamycin resistance. Antimicrobial agents and chemotherapy 40: 221-224.

Bayer AS, Speert DP, Park S, Tu J, Witt M, Nast CC \& Norman DC (1991) Functional role of mucoid exopolysaccharide (alginate) in antibiotic-induced and polymorphonuclear leukocyte-mediated killing of Pseudomonas aeruginosa. Infection and immunity 59: 302308. 
Becher A \& Schweizer HP (2000) Integration-proficient Pseudomonas aeruginosa vectors for isolation of single-copy chromosomal lac $Z$ and lux gene fusions. BioTechniques 29: 948-950, 952.

Beck BD \& Park JT (1976) Activity of three murein hydrolases during the cell division cycle of Escherichia coli $\mathrm{K}-12$ as measured in toluene-treated cells. Journal of bacteriology 126: 1250-1260.

Benson TE, Marquardt JL, Marquardt AC, Etzkorn FA \& Walsh CT (1993) Overexpression, purification, and mechanistic study of UDP-Nacetylenolpyruvylglucosamine reductase. Biochemistry 32: 2024-2030.

Bernier SP, Ha DG, Khan W, Merritt JH \& O'Toole GA (2011) Modulation of Pseudomonas aeruginosa surface-associated group behaviors by individual amino acids through c-di-GMP signaling. Research in microbiology 162: 680-688.

Bjarnsholt $\mathrm{T}$ (2013) The role of bacterial biofilms in chronic infections. APMIS. Supplementum 1-51.

Blackburn NT \& Clarke AJ (2001) Identification of four families of peptidoglycan lytic transglycosylases. Journal of molecular evolution 52: 78-84.

Blackburn NT \& Clarke AJ (2002) Characterization of soluble and membrane-bound family 3 lytic transglycosylases from Pseudomonas aeruginosa. Biochemistry 41: 10011013.

Blair JM \& Piddock LJ (2009) Structure, function and inhibition of RND efflux pumps in Gram-negative bacteria: an update. Current opinion in microbiology 12: 512-519.

Bodey GP, Bolivar R, Fainstein V \& Jadeja L (1983) Infections caused by Pseudomonas aeruginosa. Reviews of infectious diseases 5: 279-313.

Boothby D, Daneo-Moore L, Higgins ML, Coyette J \& Shockman GD (1973) Turnover of bacterial cell wall peptidoglycans. The Journal of biological chemistry 248: 2161-2169.

Borisova M, Gisin J \& Mayer C (2014) Blocking peptidoglycan recycling in Pseudomonas aeruginosa attenuates intrinsic resistance to fosfomycin. Microbial drug resistance 20: 231-237.

Borisova M, Gisin J \& Mayer C (2017) The N-acetylmuramic acid 6-phosphate phosphatase MupP completes the Pseudomonas peptidoglycan recycling pathway leading to intrinsic fosfomycin resistance. mBio 8.

Borisova M, Gaupp R, Duckworth A, et al. (2016) Peptidoglycan recycling in GramPositive bacteria is crucial for survival in stationary phase. mBio 7.

Borriello G, Richards L, Ehrlich GD \& Stewart PS (2006) Arginine or nitrate enhances antibiotic susceptibility of Pseudomonas aeruginosa in biofilms. Antimicrobial agents and chemotherapy 50: 382-384.

Boudreau MA, Fisher JF \& Mobashery S (2012) Messenger functions of the bacterial cell wall-derived muropeptides. Biochemistry 51: 2974-2990.

Bouhss A, Crouvoisier M, Blanot D \& Mengin-Lecreulx D (2004) Purification and characterization of the bacterial MraY translocase catalyzing the first membrane step of peptidoglycan biosynthesis. The Journal of biological chemistry 279: 29974-29980. 
Bouthors AT, Dagoneau-Blanchard N, Naas T, Nordmann P, Jarlier V \& Sougakoff W (1998) Role of residues 104, 164, 166, 238 and 240 in the substrate profile of PER-1 beta-lactamase hydrolysing third-generation cephalosporins. The Biochemical journal 330 ( Pt 3): 1443-1449.

Bouza E, Burillo A \& Munoz P (2002) Catheter-related infections: diagnosis and intravascular treatment. Clinical microbiology and infection : the official publication of the European Society of Clinical Microbiology and Infectious Diseases 8: 265-274.

Boyer S, Faure K, Ader F, et al. (2005) Chronic pneumonia with Pseudomonas aeruginosa and impaired alveolar fluid clearance. Respiratory research 6: 17.

Bradley DE (1980) A function of Pseudomonas aeruginosa PAO polar pili: twitching motility. Canadian journal of microbiology 26: 146-154.

Branda SS, Vik S, Friedman L \& Kolter R (2005) Biofilms: the matrix revisited. Trends in microbiology 13: 20-26.

Braun V \& Rehn K (1969) Chemical characterization, spatial distribution and function of a lipoprotein (murein-lipoprotein) of the $E$. coli cell wall. The specific effect of trypsin on the membrane structure. European journal of biochemistry / FEBS 10: 426-438.

Braun V \& Sieglin U (1970) The covalent murein-lipoprotein structure of the Escherichia coli cell wall. The attachment site of the lipoprotein on the murein. European journal of biochemistry / FEBS 13: 336-346.

Breidenstein EB, de la Fuente-Nunez C \& Hancock RE (2011) Pseudomonas aeruginosa: all roads lead to resistance. Trends in microbiology 19: 419-426.

Brenner S (1974) The genetics of Caenorhabditis elegans. Genetics 77: 71-94.

Brizio A, Conceicao T, Pimentel M, Da Silva G \& Duarte A (2006) High-level expression of IMP-5 carbapenemase owing to point mutation in the -35 promoter region of class 1 integron among Pseudomonas aeruginosa clinical isolates. International journal of antimicrobial agents 27: 27-31.

Brown K, Vial SC, Dedi N, et al. (2013) Crystal structure of the Pseudomonas aeruginosa MurG: UDP-GlcNAc substrate complex. Protein and peptide letters 20: 10021008.

Brown SP, West SA, Diggle SP \& Griffin AS (2009) Social evolution in micro-organisms and a Trojan horse approach to medical intervention strategies. Philosophical transactions of the Royal Society of London. Series B, Biological sciences 364: 31573168.

Buist G, Steen A, Kok J \& Kuipers OP (2008) LysM, a widely distributed protein motif for binding to (peptido)glycans. Molecular microbiology 68: 838-847.

Burman LG, Reichler J \& Park JT (1983) Evidence for multisite growth of Escherichia coli murein involving concomitant endopeptidase and transpeptidase activities. Journal of bacteriology 156: 386-392.

Bush K (2012) Antimicrobial agents targeting bacterial cell walls and cell membranes. Revue scientifique et technique 31: 43-56.

Bush K (2013) Introduction to Antimicrobial Therapeutics Reviews: the bacterial cell wall as an antimicrobial target. Annals of the New York Academy of Sciences 1277: v-vii. 
Bush K \& Singer SB (1989) Biochemical characteristics of extended broad spectrum beta-lactamases. Infection 17: 429-433.

Bush K, Jacoby GA \& Medeiros AA (1995) A functional classification scheme for betalactamases and its correlation with molecular structure. Antimicrobial agents and chemotherapy 39: 1211-1233.

Bycroft BW \& Shute RE (1985) The molecular basis for the mode of action of Betalactam antibiotics and mechanisms of resistance. Pharmaceutical research 2: 3-14.

Cadro D, Horan, T., Andrus, M., Dembinski, M., Edwards, J., Peavy, G., (2004) National Nosocomial Infections Surveillance (NNIS) System Report, data summary from January 1992 through June 2004, issued October 2004. American journal of infection control 32: 470-485.

Caille O, Zincke D, Merighi M, et al. (2014) Structural and functional characterization of Pseudomonas aeruginosa global regulator AmpR. Journal of bacteriology 196: 38903902.

Calcagno M, Campos PJ, Mulliert G \& Suastegui J (1984) Purification, molecular and kinetic properties of glucosamine-6-phosphate isomerase (deaminase) from Escherichia coli. Biochimica et biophysica acta 787: 165-173.

Caldwell CC, Chen Y, Goetzmann HS, et al. (2009) Pseudomonas aeruginosa exotoxin pyocyanin causes cystic fibrosis airway pathogenesis. The American journal of pathology 175: 2473-2488.

Cantarel BL, Coutinho PM, Rancurel C, Bernard T, Lombard V \& Henrissat B (2009) The Carbohydrate-Active EnZymes database (CAZy): an expert resource for glycogenomics. Nucleic acids research 37: D233-238.

Carlson CA \& Ingraham JL (1983) Comparison of denitrification by Pseudomonas stutzeri, Pseudomonas aeruginosa, and Paracoccus denitrificans. Applied and environmental microbiology 45: 1247-1253.

Carlsson M, Shukla S, Petersson AC, Segelmark M \& Hellmark T (2011) Pseudomonas aeruginosa in cystic fibrosis: pyocyanin negative strains are associated with BPI-ANCA and progressive lung disease. Journal of cystic fibrosis : official journal of the European Cystic Fibrosis Society 10: 265-271.

Carrasco-Lopez C, Rojas-Altuve A, Zhang W, et al. (2011) Crystal structures of bacterial peptidoglycan amidase AmpD and an unprecedented activation mechanism. The Journal of biological chemistry 286: 31714-31722.

Caselli D, Cesaro S, Ziino O, et al. (2010) Multidrug resistant Pseudomonas aeruginosa infection in children undergoing chemotherapy and hematopoietic stem cell transplantation. Haematologica 95: 1612-1615.

Castanheira M, Toleman MA, Jones RN, Schmidt FJ \& Walsh TR (2004) Molecular characterization of a beta-lactamase gene, bla $\mathrm{GIM}_{\mathrm{I}-1}$, encoding a new subclass of metallobeta-lactamase. Antimicrobial agents and chemotherapy 48: 4654-4661.

Cataldi TR, Bianco G, Frommberger M \& Schmitt-Kopplin P (2004) Direct analysis of selected $\mathrm{N}$-acyl-L-homoserine lactones by gas chromatography/mass spectrometry. Rapid communications in mass spectrometry : RCM 18: 1341-1344. 
Cavallari JF, Lamers RP, Scheurwater EM, Matos AL \& Burrows LL (2013) Changes to its peptidoglycan-remodeling enzyme repertoire modulate beta-lactam resistance in Pseudomonas aeruginosa. Antimicrobial agents and chemotherapy 57: 3078-3084.

CDC (2013) Antibiotic Resistance Threats in the United States, 2013. Vol. 2017 ed. ${ }^{\wedge}$ eds.), p.^pp. Atlanta: Centers for Disease Control and Prevention; 2013.

Chahboune A, Decaffmeyer M, Brasseur R \& Joris B (2005) Membrane topology of the Escherichia coli AmpG permease required for recycling of cell wall anhydromuropeptides and AmpC beta-lactamase induction. Antimicrobial agents and chemotherapy 49: 11451149.

Chaloupka J, Kreckova P \& Rihova L (1962) The mucopeptide turnover in the cell walls of growing cultures of Bacillus megaterium KM. Experientia 18: 362-363.

Chamaillard M, Hashimoto M, Horie Y, et al. (2003) An essential role for NOD1 in host recognition of bacterial peptidoglycan containing diaminopimelic acid. Nature immunology 4: 702-707.

Chan JM \& Dillard JP (2016) Neisseria gonorrhoeae crippled Its peptidoglycan fragment permease to facilitate toxic peptidoglycan monomer release. Journal of bacteriology 198: 3029-3040.

Chatterjee AN \& Park JT (1964) Biosynthesis of cell wall mucopeptide by a particulate fraction from Staphylococcus aureus. Proceedings of the National Academy of Sciences of the United States of America 51: 9-16.

Chatterjee N, Chatterjee C, Ghosh S, Mukhopadhyay M, Brahmachari R \& Patar K (2016) Pattern of urinary antibiograms in a tertiary care hospital of eastern India. The Journal of the Association of Physicians of India 64: 26-30.

Chen W, Zhang YM \& Davies C (2017) Penicillin-Binding Protein 3 is essential for growth of Pseudomonas aeruginosa. Antimicrobial agents and chemotherapy 61.

Cheng K, Smyth RL, Govan JR, et al. (1996) Spread of beta-lactam-resistant Pseudomonas aeruginosa in a cystic fibrosis clinic. Lancet 348: 639-642.

Cheng Q \& Park JT (2002) Substrate specificity of the AmpG permease required for recycling of cell wall anhydro-muropeptides. Journal of bacteriology 184: 6434-6436.

Cheng Q, Li H, Merdek K \& Park JT (2000) Molecular characterization of the beta-Nacetylglucosaminidase of Escherichia coli and its role in cell wall recycling. Journal of bacteriology 182: 4836-4840.

Chuanchuen R, Narasaki CT \& Schweizer HP (2002) The MexJK efflux pump of Pseudomonas aeruginosa requires OprM for antibiotic efflux but not for efflux of triclosan. Journal of bacteriology 184: 5036-5044.

Clarke TB, Kawai F, Park SY, Tame JR, Dowson CG \& Roper DI (2009) Mutational analysis of the substrate specificity of Escherichia coli penicillin binding protein 4. Biochemistry 48: 2675-2683.

Collia D, Bannister TD, Tan $\mathrm{H}$, et al. (2018) A rapid phenotypic whole-cell screening approach for the identification of small-molecule inhibitors that counter beta-lactamase resistance in Pseudomonas aeruginosa. SLAS discovery : advancing life sciences $R \& D$ 23: $55-64$. 
Cookson BT, Tyler AN \& Goldman WE (1989) Primary structure of the peptidoglycanderived tracheal cytotoxin of Bordetella pertussis. Biochemistry 28: 1744-1749.

Costerton JW, Stewart PS \& Greenberg EP (1999) Bacterial biofilms: a common cause of persistent infections. Science 284: 1318-1322.

Cox CD \& Adams P (1985) Siderophore activity of pyoverdin for Pseudomonas aeruginosa. Infection and immunity 48: 130-138.

Coyne S, Courvalin P \& Galimand M (2010) Acquisition of multidrug resistance transposon Tn6061 and IS6100-mediated large chromosomal inversions in Pseudomonas aeruginosa clinical isolates. Microbiology 156: 1448-1458.

Craney A, Hohenauer T, Xu Y, Navani NK, Li Y \& Nodwell J (2007) A synthetic luxCDABE gene cluster optimized for expression in high-GC bacteria. Nucleic acids research 35: e46.

Crouch Brewer S, Wunderink RG, Jones CB \& Leeper KV, Jr. (1996) Ventilatorassociated pneumonia due to Pseudomonas aeruginosa. Chest 109: 1019-1029.

Cunrath O, Gasser V, Hoegy F, Reimmann C, Guillon L \& Schalk IJ (2015) A cell biological view of the siderophore pyochelin iron uptake pathway in Pseudomonas aeruginosa. Environmental microbiology 17: 171-185.

Dahl U, Jaeger T, Nguyen BT, Sattler JM \& Mayer C (2004) Identification of a phosphotransferase system of Escherichia coli required for growth on $\mathrm{N}$-acetylmuramic acid. Journal of bacteriology 186: 2385-2392.

Dale JW \& Smith JT (1972) A direct comparison of two unusual R-factor-mediated lactamases. The Biochemical journal 128: 173-174.

Dale RM, Schnell G \& Wong JP (2004) Therapeutic efficacy of "nubiotics" against burn wound infection by Pseudomonas aeruginosa. Antimicrobial agents and chemotherapy 48: 2918-2923.

Dan S, Shah A, Justo JA, Bookstaver PB, Kohn J, Albrecht H \& Al-Hasan MN (2016) Prediction of fluoroquinolone resistance in Gram-negative bacteria causing bloodstream infections. Antimicrobial agents and chemotherapy 60: 2265-2272.

Danel F, Hall LM, Gur D \& Livermore DM (1995) OXA-14, another extended-spectrum variant of OXA-10 (PSE-2) beta-lactamase from Pseudomonas aeruginosa. Antimicrobial agents and chemotherapy 39: 1881-1884.

Danel F, Hall LM, Gur D \& Livermore DM (1998) OXA-16, a further extended-spectrum variant of OXA-10 beta-lactamase, from two Pseudomonas aeruginosa isolates. Antimicrobial agents and chemotherapy 42: 3117-3122.

Danel F, Hall LM, Duke B, Gur D \& Livermore DM (1999) OXA-17, a further extendedspectrum variant of OXA-10 beta-lactamase, isolated from Pseudomonas aeruginosa. Antimicrobial agents and chemotherapy 43: 1362-1366.

Dart JK \& Seal DV (1988) Pathogenesis and therapy of Pseudomonas aeruginosa keratitis. Eye 2 Suppl: S46-55.

Dasgupta N, Wolfgang MC, Goodman AL, Arora SK, Jyot J, Lory S \& Ramphal R (2003) A four-tiered transcriptional regulatory circuit controls flagellar biogenesis in Pseudomonas aeruginosa. Mol Microbiol 50: 809-824. 
Datta N \& Kontomichalou P (1965) Penicillinase synthesis controlled by infectious R factors in Enterobacteriaceae. Nature 208: 239-241.

Davey ME, Caiazza NC \& O'Toole GA (2003) Rhamnolipid surfactant production affects biofilm architecture in Pseudomonas aeruginosa PAO1. Journal of bacteriology 185: 1027-1036.

Davies DG, Parsek MR, Pearson JP, Iglewski BH, Costerton JW \& Greenberg EP (1998) The involvement of cell-to-cell signals in the development of a bacterial biofilm. Science 280: 295-298.

Davies J \& Davies D (2010) Origins and evolution of antibiotic resistance. Microbiology and molecular biology reviews : MMBR 74: 417-433.

Davies J, Gilbert W \& Gorini L (1964) Streptomycin, suppression, and the code. Proceedings of the National Academy of Sciences of the United States of America 51: 883-890.

Davies JC (2002) Pseudomonas aeruginosa in cystic fibrosis: pathogenesis and persistence. Paediatric respiratory reviews 3: 128-134.

Davies KJ, Lloyd D \& Boddy L (1989) The effect of oxygen on denitrification in Paracoccus denitrificans and Pseudomonas aeruginosa. Journal of general microbiology 135: 2445-2451.

De Pedro MA, Schwarz, U., Nishimura, Y and Hirota, Y. (1980) On the biological role of penicllin-binding proteins 4 and 5. FEMS Microbiology Letters 9: 219-221.

Dekimpe V \& Deziel E (2009) Revisiting the quorum-sensing hierarchy in Pseudomonas aeruginosa: the transcriptional regulator $\mathrm{RhIR}$ regulates LasR-specific factors. Microbiology 155: 712-723.

Denome SA, Elf PK, Henderson TA, Nelson DE \& Young KD (1999) Escherichia coli mutants lacking all possible combinations of eight penicillin binding proteins: viability, characteristics, and implications for peptidoglycan synthesis. Journal of bacteriology 181: 3981-3993.

Dhar S, Kumari H, Balasubramanian D \& Mathee K (2018) Cell-wall recycling and synthesis in Escherichia coli and Pseudomonas aeruginosa - their role in the development of resistance. J Med Microbiol 67: 1-21.

Dietrich LE, Price-Whelan A, Petersen A, Whiteley M \& Newman DK (2006) The phenazine pyocyanin is a terminal signalling factor in the quorum sensing network of Pseudomonas aeruginosa. Molecular microbiology 61: 1308-1321.

Dietz H, Pfeifle D \& Wiedemann B (1997) The signal molecule for beta-lactamase induction in Enterobacter cloacae is the anhydromuramyl-pentapeptide. Antimicrobial agents and chemotherapy 41: 2113-2120.

Dijkstra AJ \& Keck W (1996) Identification of new members of the lytic transglycosylase family in Haemophilus influenzae and Escherichia coli. Microbial drug resistance 2: 141145.

Dik DA, Dominguez-Gil T, Lee $\mathrm{M}$, et al. (2017) Muropeptide binding and the X-ray structure of the effector domain of the transcriptional regulator AmpR of Pseudomonas aeruginosa. Journal of the American Chemical Society 139: 1448-1451. 
Doern CD (2014) When does 2 plus 2 equal 5? A review of antimicrobial synergy testing. Journal of clinical microbiology 52: 4124-4128.

Doggett RG, Harrison GM \& Wallis ES (1964) Comparison of some properties of Pseudomonas aeruginosa isolated from infections in persons with and without cystic fibrosis. Journal of bacteriology 87: 427-431.

Doyle RJ, Chaloupka J \& Vinter V (1988) Turnover of cell walls in microorganisms. Microbiological reviews 52: 554-567.

Doyle TB, Hawkins AC \& McCarter LL (2004) The complex flagellar torque generator of Pseudomonas aeruginosa. Journal of bacteriology 186: 6341-6350.

Driscoll JA, Brody SL \& Kollef MH (2007) The epidemiology, pathogenesis and treatment of Pseudomonas aeruginosa infections. Drugs 67: 351-368.

Drlica K \& Zhao X (1997) DNA gyrase, topoisomerase IV, and the 4-quinolones. Microbiology and molecular biology reviews : MMBR 61: 377-392.

Drlica K, Malik M, Kerns RJ \& Zhao X (2008) Quinolone-mediated bacterial death. Antimicrobial agents and chemotherapy 52: 385-392.

Duan K, Dammel C, Stein J, Rabin H \& Surette MG (2003) Modulation of Pseudomonas aeruginosa gene expression by host microflora through interspecies communication. Molecular microbiology 50: 1477-1491.

Dufresne J, Vezina G \& Levesque RC (1988) Cloning and expression of the imipenemhydrolyzing beta-lactamase operon from Pseudomonas maltophilia in Escherichia coli. Antimicrobial agents and chemotherapy 32: 819-826.

Duncan K, van Heijenoort J \& Walsh CT (1990) Purification and characterization of the D-alanyl-D-alanine-adding enzyme from Escherichia coli. Biochemistry 29: 2379-2386.

Eckert C, Lecerf M, Dubost L, Arthur M \& Mesnage S (2006) Functional analysis of AtIA, the major $\mathrm{N}$-acetylglucosaminidase of Enterococcus faecalis. Journal of bacteriology 188: 8513-8519.

Eibergen NR, Moore JD, Mattmann ME \& Blackwell HE (2015) Potent and selective modulation of the RhIR quorum sensing receptor by using non-native ligands: An emerging target for virulence control in Pseudomonas aeruginosa. Chembiochem : a European journal of chemical biology 16: 2348-2356.

El Zoeiby A, Sanschagrin F, Lamoureux J, Darveau A \& Levesque RC (2000) Cloning, over-expression and purification of Pseudomonas aeruginosa murC encoding uridine diphosphate $\mathrm{N}$-acetylmuramate: L-alanine ligase. FEMS microbiology letters 183: 281288.

El Zoeiby A, Sanschagrin F, Havugimana PC, Garnier A \& Levesque RC (2001) In vitro reconstruction of the biosynthetic pathway of peptidoglycan cytoplasmic precursor in Pseudomonas aeruginosa. FEMS microbiology letters 201: 229-235.

Elhenawy W, Davis RM, Fero J, Salama NR, Felman MF \& Ruiz N (2016) The O-antigen flippase Wzk can substitute for MurJ in peptidoglycan synthesis in Helicobacter pylori and Escherichia coli. PloS one 11: e0161587. 
Emerson J, Rosenfeld M, McNamara S, Ramsey B \& Gibson RL (2002) Pseudomonas aeruginosa and other predictors of mortality and morbidity in young children with cystic fibrosis. Pediatric pulmonology 34: 91-100.

Eng RH, Bishburg E, Smith SM, Geller H \& Kapila R (1986) Bacteremia and fungemia in patients with acquired immune deficiency syndrome. American journal of clinical pathology 86: 105-107.

Engel H, Smink AJ, van Wijngaarden L \& Keck W (1992) Murein-metabolizing enzymes from Escherichia coli: existence of a second lytic transglycosylase. Journal of bacteriology 174: 6394-6403.

Engel LS, Hill JM, Caballero AR, Green LC \& O'Callaghan RJ (1998) Protease IV, a unique extracellular protease and virulence factor from Pseudomonas aeruginosa. The Journal of biological chemistry 273: 16792-16797.

Essar DW, Eberly L, Hadero A \& Crawford IP (1990) Identification and characterization of genes for a second anthranilate synthase in Pseudomonas aeruginosa: interchangeability of the two anthranilate synthases and evolutionary implications. Journal of bacteriology 172: 884-900.

Estahbanati HK, Kashani PP \& Ghanaatpisheh F (2002) Frequency of Pseudomonas aeruginosa serotypes in burn wound infections and their resistance to antibiotics. Burns : journal of the International Society for Burn Injuries 28: 340-348.

EUCAST (2000) EUCAST Definitive Document E.DEF 3.1, June 2000: Determination of minimum inhibitory concentrations (MICs) of antibacterial agents by agar dilution. Clinical microbiology and infection : the official publication of the European Society of Clinical Microbiology and Infectious Diseases 6: 509-515.

Evans LR \& Linker A (1973) Production and characterization of the slime polysaccharide of Pseudomonas aeruginosa. Journal of bacteriology 116: 915-924.

Everett J, Turner K, Cai Q, Gordon V, Whiteley M \& Rumbaugh K (2017) Arginine Is a Critical Substrate for the Pathogenesis of Pseudomonas aeruginosa in Burn Wound Infections. mBio 8.

Fairfax MR, Queenan AM, Lephart PR, et al. (2011) Detection of 2 SME-1 carbapenemase-producing Serratia marcescens in Detroit. Diagnostic microbiology and infectious disease 71: 325-326.

Fajardo A, Hernando-Amado S, Oliver A, Ball G, Filloux A \& Martinez JL (2014) Characterization of a novel $\mathrm{Zn}(2)(+)$-dependent intrinsic imipenemase from Pseudomonas aeruginosa. The Journal of antimicrobial chemotherapy 69: 2972-2978.

Falagas ME \& Kasiakou SK (2005) Colistin: the revival of polymyxins for the management of multidrug-resistant Gram-negative bacterial infections. Clinical infectious diseases : an official publication of the Infectious Diseases Society of America 40: 13331341.

Favero MS, Carson LA, Bond WW \& Petersen NJ (1971) Pseudomonas aeruginosa: growth in distilled water from hospitals. Science 173: 836-838.

Fein JE (1979) Possible involvement of bacterial autolytic enzymes in flagellar morphogenesis. Journal of bacteriology 137: 933-946. 
Feldman M, Bryan R, Rajan S, Scheffler L, Brunnert S, Tang H \& Prince A (1998) Role of flagella in pathogenesis of Pseudomonas aeruginosa pulmonary infection. Infection and immunity 66: 43-51.

Feunou PF, Ismaili J, Debrie AS, et al. (2008) Genetic stability of the live attenuated Bordetella pertussis vaccine candidate BPZE1. Vaccine 26: 5722-5727.

Figurski DH \& Helinski DR (1979) Replication of an origin-containing derivative of plasmid RK2 dependent on a plasmid function provided in trans. Proc Natl Acad Sci U S A 76: 1648-1652.

Finan TM, Kunkel B, De Vos GF \& Signer ER (1986) Second symbiotic megaplasmid in Rhizobium meliloti carrying exopolysaccharide and thiamine synthesis genes. J Bacteriol 167: 66-72.

Finck-Barbancon V, Goranson J, Zhu L, et al. (1997) ExoU expression by Pseudomonas aeruginosa correlates with acute cytotoxicity and epithelial injury. Molecular microbiology 25: 547-557.

Finn RD, Coggill P, Eberhardt RY, et al. (2016) The Pfam protein families database: towards a more sustainable future. Nucleic acids research 44: D279-285.

Fisher JF \& Mobashery S (2014) The sentinel role of peptidoglycan recycling in the betalactam resistance of the Gram-negative Enterobacteriaceae and Pseudomonas aeruginosa. Bioorganic chemistry 56: 41-48.

Fisher JF, Meroueh SO \& Mobashery S (2005) Bacterial resistance to beta-lactam antibiotics: compelling opportunism, compelling opportunity. Chemical reviews 105: 395424.

Fito-Boncompte L, Chapalain A, Bouffartigues E, et al. (2011) Full virulence of Pseudomonas aeruginosa requires OprF. Infection and immunity 79: 1176-1186.

Fleeman R, LaVoi TM, Santos RG, et al. (2015) Combinatorial libraries as a tool for the discovery of novel, broad-spectrum antibacterial agents targeting the ESKAPE pathogens. Journal of medicinal chemistry 58: 3340-3355.

Flemming HC \& Wingender J (2010) The biofilm matrix. Nature reviews. Microbiology 8 : 623-633.

Flume PA, Mogayzel PJ, Jr., Robinson KA, Goss CH, Rosenblatt RL, Kuhn RJ \& Marshall BC (2009) Cystic fibrosis pulmonary guidelines: treatment of pulmonary exacerbations. American journal of respiratory and critical care medicine 180: 802-808.

Folkesson A, Eriksson S, Andersson M, Park JT \& Normark S (2005) Components of the peptidoglycan-recycling pathway modulate invasion and intracellular survival of Salmonella enterica serovar Typhimurium. Cellular microbiology 7: 147-155.

Fothergill JL, Winstanley C \& James CE (2012) Novel therapeutic strategies to counter Pseudomonas aeruginosa infections. Expert review of anti-infective therapy 10: 219-235.

Frank DW (1997) The exoenzyme S regulon of Pseudomonas aeruginosa. Molecular microbiology 26: 621-629.

Freire P, Moreira RN \& Arraiano CM (2009) BolA inhibits cell elongation and regulates MreB expression levels. Journal of molecular biology 385: 1345-1351. 
Friedman L \& Kolter R (2004) Genes involved in matrix formation in Pseudomonas aeruginosa PA14 biofilms. Molecular microbiology 51: 675-690.

Fujimoto Y \& Fukase K (2011) Structures, synthesis, and human Nod1 stimulation of immunostimulatory bacterial peptidoglycan fragments in the environment. Journal of natural products 74 : $518-525$.

Fumeaux C \& Bernhardt TG (2017) Identification of MupP as a new peptidoglycan recycling factor and antibiotic resistance determinant in Pseudomonas aeruginosa. mBio 8.

Furste JP, Pansegrau W, Frank R, Blocker H, Scholz P, Bagdasarian M \& Lanka E (1986) Molecular cloning of the plasmid RP4 primase region in a multi-host-range tac $P$ expression vector. Gene 48: 119-131.

Furukawa S, Kuchma SL \& O'Toole GA (2006) Keeping their options open: acute versus persistent infections. Journal of bacteriology 188: 1211-1217.

Galloway DR (1991) Pseudomonas aeruginosa elastase and elastolysis revisited: recent developments. Molecular microbiology 5: 2315-2321.

Gambello MJ \& Iglewski BH (1991) Cloning and characterization of the Pseudomonas aeruginosa las $R$ gene, a transcriptional activator of elastase expression. Journal of bacteriology 173: 3000-3009.

Gambello MJ, Kaye S \& Iglewski BH (1993) LasR of Pseudomonas aeruginosa is a transcriptional activator of the alkaline protease gene (apr) and an enhancer of exotoxin A expression. Infection and immunity 61: 1180-1184.

Garau J \& Gomez L (2003) Pseudomonas aeruginosa pneumonia. Current opinion in infectious diseases 16: 135-143.

Garcia DL \& Dillard JP (2008) Mutations in ampG or $a m p D$ affect peptidoglycan fragment release from Neisseria gonorrhoeae. Journal of bacteriology 190: 3799-3807.

Gaynes R \& Edwards JR (2005) Overview of nosocomial infections caused by Gramnegative bacilli. Clinical infectious diseases : an official publication of the Infectious Diseases Society of America 41: 848-854.

Gellatly SL \& Hancock RE (2013) Pseudomonas aeruginosa: new insights into pathogenesis and host defenses. Pathogens and disease 67: 159-173.

Gibson DG, Young L, Chuang RY, Venter JC, Hutchison CA, 3rd \& Smith HO (2009) Enzymatic assembly of DNA molecules up to several hundred kilobases. Nat Methods 6: 343-345.

Girardin SE, Travassos LH, Herve M, et al. (2003) Peptidoglycan molecular requirements allowing detection by Nod1 and Nod2. The Journal of biological chemistry 278: 41702-41708.

Girlich D, Naas T \& Nordmann P (2004) Biochemical characterization of the naturally occurring oxacillinase OXA-50 of Pseudomonas aeruginosa. Antimicrobial agents and chemotherapy 48: 2043-2048.

Gisin J, Schneider A, Nagele B, Borisova M \& Mayer C (2013) A cell wall recycling shortcut that bypasses peptidoglycan de novo biosynthesis. Nature chemical biology 9 : 491-493. 
Glauner B (1988) Separation and quantification of muropeptides with high-performance liquid chromatography. Analytical biochemistry 172: 451-464.

Glauner B, Holtje JV \& Schwarz U (1988) The composition of the murein of Escherichia coli. The Journal of biological chemistry 263: 10088-10095.

Godfrey AJ, Bryan LE \& Rabin HR (1981) beta-Lactam-resistant Pseudomonas aeruginosa with modified penicillin-binding proteins emerging during cystic fibrosis treatment. Antimicrobial agents and chemotherapy 19: 705-711.

Goffin C \& Ghuysen JM (1998) Multimodular penicillin-binding proteins: an enigmatic family of orthologs and paralogs. Microbiology and molecular biology reviews : MMBR 62: 1079-1093.

Goldman WE \& Herwaldt LA (1985) Bordetella pertussis tracheal cytotoxin. Developments in biological standardization 61: 103-111.

Goldman WE \& Cookson BT (1988) Structure and functions of the Bordetella tracheal cytotoxin. The Tokai journal of experimental and clinical medicine 13 Suppl: 187-191.

Goodell EW (1985) Recycling of murein by Escherichia coli. Journal of bacteriology 163: 305-310.

Goodell EW \& Schwarz U (1985) Release of cell wall peptides into culture medium by exponentially growing Escherichia coli. Journal of bacteriology 162: 391-397.

Goodell EW \& Higgins CF (1987) Uptake of cell wall peptides by Salmonella typhimurium and Escherichia coli. Journal of bacteriology 169: 3861-3865.

Goodman AL, Kulasekara B, Rietsch A, Boyd D, Smith RS \& Lory S (2004) A signaling network reciprocally regulates genes associated with acute infection and chronic persistence in Pseudomonas aeruginosa. Developmental cell 7: 745-754.

Goodman AL, Merighi M, Hyodo M, Ventre I, Filloux A \& Lory S (2009) Direct interaction between sensor kinase proteins mediates acute and chronic disease phenotypes in a bacterial pathogen. Genes \& development 23: 249-259.

Gotoh N, Wakebe H \& Nishino T (1989) Ultrastructural aspects of fragility of Pseudomonas aeruginosa outer membrane devoid of protein F. FEMS microbiology letters 50: 51-53.

Gotoh N, Tsujimoto H, Tsuda M, et al. (1998) Characterization of the MexC-MexD-OprJ multidrug efflux system in DeltamexA-mexB-oprM mutants of Pseudomonas aeruginosa. Antimicrobial agents and chemotherapy 42: 1938-1943.

Govan JR \& Harris GS (1986) Pseudomonas aeruginosa and cystic fibrosis: unusual bacterial adaptation and pathogenesis. Microbiological sciences 3: 302-308.

Guinote IB, Matos RG, Freire P \& Arraiano CM (2011) BolA affects cell growth, and binds to the promoters of penicillin-binding proteins 5 and 6 and regulates their expression. Journal of microbiology and biotechnology 21: 243-251.

Gupta SK, Berk RS, Masinick S \& Hazlett LD (1994) Pili and lipopolysaccharide of Pseudomonas aeruginosa bind to the glycolipid asialo GM1. Infection and immunity 62 : 4572-4579. 
Hancock RE (1998) Resistance mechanisms in Pseudomonas aeruginosa and other nonfermentative gram-negative bacteria. Clinical infectious diseases : an official publication of the Infectious Diseases Society of America 27 Suppl 1: S93-99.

Hancock RE, Decad GM \& Nikaido H (1979) Identification of the protein producing transmembrane diffusion pores in the outer membrane of Pseudomonas aeruginosa PA01. Biochimica et biophysica acta 554: 323-331.

Handfield J, Gagnon L, Dargis M \& Huletsky A (1997) Sequence of the ponA gene and characterization of the penicillin-binding protein 1A of Pseudomonas aeruginosa PAO1. Gene 199: 49-56.

Hashimoto W, Ochiai A, Momma K, Itoh T, Mikami B, Maruyama Y \& Murata K (2009) Crystal structure of the glycosidase family 73 peptidoglycan hydrolase FlgJ. Biochemical and biophysical research communications 381: 16-21.

Hassan MT, van der Lelie D, Springael D, Romling U, Ahmed N \& Mergeay M (1999) Identification of a gene cluster, czr, involved in cadmium and zinc resistance in Pseudomonas aeruginosa. Gene 238: 417-425.

Hassett DJ, Charniga L, Bean K, Ohman DE \& Cohen MS (1992) Response of Pseudomonas aeruginosa to pyocyanin: mechanisms of resistance, antioxidant defenses, and demonstration of a manganese-cofactored superoxide dismutase. Infection and immunity 60: 328-336.

Hauser AR (2009) The type III secretion system of Pseudomonas aeruginosa: infection by injection. Nature reviews. Microbiology 7: 654-665.

Heck LW, Alarcon PG, Kulhavy RM, Morihara K, Russell MW \& Mestecky JF (1990) Degradation of $\lg \mathrm{A}$ proteins by Pseudomonas aeruginosa elastase. Journal of immunology 144: 2253-2257.

Heidrich C, Ursinus A, Berger J, Schwarz H \& Holtje JV (2002) Effects of multiple deletions of murein hydrolases on viability, septum cleavage, and sensitivity to large toxic molecules in Escherichia coli. Journal of bacteriology 184: 6093-6099.

Heidrich C, Templin MF, Ursinus A, et al. (2001) Involvement of N-acetylmuramyl-Lalanine amidases in cell separation and antibiotic-induced autolysis of Escherichia coli. Molecular microbiology 41: 167-178.

Heilmann HD (1972) On the peptidoglycan of the cell walls of Pseudomonas aeruginosa. European journal of biochemistry 31: 456-463.

Heilmann HD (1974) On the peptidoglycan of the cell walls of Pseudomonas aeruginosa. Structure of the peptide side chains. European journal of biochemistry 43: 35-38.

Heimer SR, Evans DJ, Stern ME, Barbieri JT, Yahr T \& Fleiszig SM (2013) Pseudomonas aeruginosa utilizes the type III secreted toxin ExoS to avoid acidified compartments within epithelial cells. PloS one 8: e73111.

Henderson TA, Templin M \& Young KD (1995) Identification and cloning of the gene encoding penicillin-binding protein 7 of Escherichia coli. Journal of bacteriology 177: 2074-2079.

Henrichsen J (1972) Bacterial surface translocation: a survey and a classification. Bacteriological reviews 36: 478-503. 
Herlihey FA, Moynihan PJ \& Clarke AJ (2014) The essential protein for bacterial flagella formation FlgJ functions as a beta- $\mathrm{N}$-acetylglucosaminidase. The Journal of biological chemistry 289: 31029-31042.

Hernandez A, Sanchez MB \& Martinez JL (2011) Quinolone resistance: much more than predicted. Frontiers in microbiology 2: 22.

Herve M, Boniface A, Gobec S, Blanot D \& Mengin-Lecreulx D (2007) Biochemical characterization and physiological properties of Escherichia coli UDP-Nacetylmuramate:L-alanyl-gamma-D-glutamyl-meso-diaminopimelate ligase. Journal of bacteriology 189: 3987-3995.

Hesek D, Lee M, Zhang W, Noll BC \& Mobashery S (2009) Total synthesis of Nacetylglucosamine-1,6-anhydro-N-acetylmuramylpentapeptide and evaluation of its turnover by AmpD from Escherichia coli. Journal of the American Chemical Society 131: 5187-5193.

Hirano T, Minamino T \& Macnab RM (2001) The role in flagellar rod assembly of the Nterminal domain of Salmonella FlgJ, a flagellum-specific muramidase. J Mol Biol 312: 359-369.

Hoang TT, Karkhoff-Schweizer RR, Kutchma AJ \& Schweizer HP (1998) A broad-hostrange Flp-FRT recombination system for site-specific excision of chromosomally-located DNA sequences: application for isolation of unmarked Pseudomonas aeruginosa mutants. Gene 212: 77-86.

Hobden JA (2002) Pseudomonas aeruginosa proteases and corneal virulence. DNA and cell biology 21: 391-396.

Hoiby N (1974) Pseudomonas aeruginosa infection in cystic fibrosis. Relationship between mucoid strains of Pseudomonas aeruginosa and the humoral immune response. Acta pathologica et microbiologica Scandinavica. Section B: Microbiology and immunology 82: 551-558.

Holloway BW (1955) Genetic recombination in Pseudomonas aeruginosa. Journal of general microbiology 13: 572-581.

Holloway BW \& Morgan AF (1986) Genome organization in Pseudomonas. Annual review of microbiology 40: 79-105.

Holloway BW, Krishnapillai V \& Morgan AF (1979) Chromosomal genetics of Pseudomonas. Microbiological reviews 43: 73-102.

Holtje JV (1998) Growth of the stress-bearing and shape-maintaining murein sacculus of Escherichia coli. Microbiology and molecular biology reviews : MMBR 62: 181-203.

Holtje JV, Mirelman D, Sharon N \& Schwarz U (1975) Novel type of murein transglycosylase in Escherichia coli. Journal of bacteriology 124: 1067-1076.

Holtje JV, Kopp U, Ursinus A \& Wiedemann B (1994) The negative regulator of betalactamase induction $\mathrm{AmpD}$ is a $\mathrm{N}$-acetyl-anhydromuramyl-L-alanine amidase. FEMS microbiology letters 122: 159-164.

Honore N, Nicolas MH \& Cole ST (1989) Regulation of enterobacterial cephalosporinase production: the role of a membrane-bound sensory transducer. Molecular microbiology 3: $1121-1130$. 
Hooper DC \& Jacoby GA (2015) Mechanisms of drug resistance: quinolone resistance. Annals of the New York Academy of Sciences 1354: 12-31.

Horsch M, Hoesch L, Vasella A \& Rast DM (1991) N-acetylglucosaminono-1,5-lactone oxime and the corresponding (phenylcarbamoyl)oxime. Novel and potent inhibitors of beta-N-acetylglucosaminidase. European journal of biochemistry 197: 815-818.

Hou JP (1977) The chemical constituents of ginseng plants. Comparative medicine East and West 5: 123-145.

Hrebenda $J$ (1979) Mutants of Escherichia coli with altered level of beta-Nacetylglucosaminidase activities. Acta microbiologica Polonica 28: 53-62.

Huang YW, Lin CW, Hu RM, Lin YT, Chung TC \& Yang TC (2010) AmpN-AmpG operon is essential for expression of L1 and L2 beta-lactamases in Stenotrophomonas maltophilia. Antimicrobial agents and chemotherapy 54: 2583-2589.

Humphrey W, Dalke A \& Schulten K (1996) VMD: visual molecular dynamics. Journal of molecular graphics 14: 33-38, 27-38.

lino T (1974) Assembly of Salmonella flagellin in vitro and in vivo. Journal of supramolecular structure 2: 372-384.

Ishino F, Mitsui K, Tamaki S \& Matsuhashi M (1980) Dual enzyme activities of cell wall peptidoglycan synthesis, peptidoglycan transglycosylase and penicillin-sensitive transpeptidase, in purified preparations of Escherichia coli penicillin-binding protein $1 \mathrm{~A}$. Biochemical and biophysical research communications 97: 287-293.

Izaki K, Matsuhashi M \& Strominger JL (1966) Glycopeptide transpeptidase and Dalanine carboxypeptidase: penicillin-sensitive enzymatic reactions. Proceedings of the National Academy of Sciences of the United States of America 55: 656-663.

J. F. Fisher SM (2015) Bacterial Cell Wall: Morphology and Biochemistry

Vol. 3rd edition ed.^eds.), p.^pp. pp. 221. CRC Press, Boca Raton, FL.

Jacobs C, Frere JM \& Normark S (1997) Cytosolic intermediates for cell wall biosynthesis and degradation control inducible beta-lactam resistance in Gram-negative bacteria. Cell 88: 823-832.

Jacobs C, Huang LJ, Bartowsky E, Normark S \& Park JT (1994) Bacterial cell wall recycling provides cytosolic muropeptides as effectors for beta-lactamase induction. The EMBO journal 13: 4684-4694.

Jacobs $C$, Joris $B$, Jamin $M$, et al. (1995) AmpD, essential for both beta-lactamase regulation and cell wall recycling, is a novel cytosolic $\mathrm{N}$-acetylmuramyl-L-alanine amidase. Molecular microbiology 15: 553-559.

Jacoby GA (2009) AmpC beta-lactamases. Clinical microbiology reviews 22: 161-182, Table of Contents.

Jacoby GA, Blaser MJ, Santanam P, Hachler H, Kayser FH, Hare RS \& Miller GH (1990) Appearance of amikacin and tobramycin resistance due to 4'-aminoglycoside nucleotidyltransferase [ANT(4')-II] in Gram-negative pathogens. Antimicrobial agents and chemotherapy 34: 2381-2386.

Janeway CA, Jr. (1989) Approaching the asymptote? Evolution and revolution in immunology. Cold Spring Harbor symposia on quantitative biology 54 Pt 1: 1-13. 
Jansen G, Mahrt N, Tueffers L, et al. (2016) Association between clinical antibiotic resistance and susceptibility of Pseudomonas in the cystic fibrosis lung. Evolution, medicine, and public health 2016: 182-194.

Jennings LK, Storek KM, Ledvina HE, et al. (2015) Pel is a cationic exopolysaccharide that cross-links extracellular DNA in the Pseudomonas aeruginosa biofilm matrix. Proceedings of the National Academy of Sciences of the United States of America 112: 11353-11358.

Johnson JW, Fisher JF \& Mobashery S (2013) Bacterial cell-wall recycling. Annals of the New York Academy of Sciences 1277: 54-75.

Jones CJ, Homma M \& Macnab RM (1989) L-, P-, and M-ring proteins of the flagellar basal body of Salmonella typhimurium: gene sequences and deduced protein sequences. Journal of bacteriology 171: 3890-3900.

Jorgensen JH (1993) Antimicrobial susceptibility testing of bacteria that grow aerobically. Infect Dis Clin North Am 7: 393-409.

Jorgenson MA, Chen Y, Yahashiri A, Popham DL \& Weiss DS (2014) The bacterial septal ring protein RlpA is a lytic transglycosylase that contributes to rod shape and daughter cell separation in Pseudomonas aeruginosa. Molecular microbiology 93: 113128.

Juan C, Moya B, Perez JL \& Oliver A (2006) Stepwise upregulation of the Pseudomonas aeruginosa chromosomal cephalosporinase conferring high-level beta-lactam resistance involves three AmpD homologues. Antimicrobial agents and chemotherapy 50: 17801787.

Juan C, Macia MD, Gutierrez O, Vidal C, Perez JL \& Oliver A (2005) Molecular mechanisms of beta-lactam resistance mediated by AmpC hyperproduction in Pseudomonas aeruginosa clinical strains. Antimicrobial agents and chemotherapy 49: 4733-4738.

K. Yanagihara JK, S. Kohno (2001) Diffuse panbronchiolitis-pathophysiology and treatment mechanisms. International Journal of Antimicrobial Agents 18: S83-S87.

Kaczor AA, Polski A, Sobotka-Polska K, Pachuta-Stec A, Makarska-Bialokoz M \& Pitucha M (2017) Novel antibacterial compounds and their drug targets - successes and challenges. Current medicinal chemistry 24: 1948-1982.

Kalva S, Boeke JD \& Mita P (2018) Gibson Deletion: a novel application of isothermal in vitro recombination. Biological procedures online 20: 2.

Kamada Y, Nagaretani H, Tamura S, et al. (2001) Vascular endothelial dysfunction resulting from L-arginine deficiency in a patient with lysinuric protein intolerance. The Journal of clinical investigation 108: 717-724.

Kaneko K, Okamoto R, Nakano R, Kawakami S \& Inoue M (2005) Gene mutations responsible for overexpression of $\mathrm{AmpC}$ beta-lactamase in some clinical isolates of Enterobacter cloacae. Journal of clinical microbiology 43: 2955-2958.

Kang Cl, Kim SH, Kim HB, et al. (2003) Pseudomonas aeruginosa bacteremia: risk factors for mortality and influence of delayed receipt of effective antimicrobial therapy on clinical outcome. Clinical infectious diseases : an official publication of the Infectious Diseases Society of America 37: 745-751. 
Kanj SS \& Kanafani ZA (2011) Current concepts in antimicrobial therapy against resistant Gram-negative organisms: extended-spectrum beta-lactamase-producing Enterobacteriaceae, carbapenem-resistant Enterobacteriaceae, and multidrug-resistant Pseudomonas aeruginosa. Mayo Clinic proceedings 86: 250-259.

Kapatral V ZA, Kamath S, Chugani S (2000) Pseudomonas.

. ed.^eds.), p.^pp. Academic Press,

, New York, NY.

Karim A, Poirel L, Nagarajan S \& Nordmann P (2001) Plasmid-mediated extendedspectrum beta-lactamase (CTX-M-3 like) from India and gene association with insertion sequence ISEcp1. FEMS microbiology letters 201: 237-241.

Kaya S, Araki $Y$ \& Ito $E$ (1989) The structure of the O-specific chain of lipopolysaccharide from Pseudomonas aeruginosa IID 1012 (ATCC 27588). Journal of biochemistry 105: 29-34.

Kearns DB (2010) A field guide to bacterial swarming motility. Nature reviews. Microbiology 8: 634-644.

Keep NH, Ward JM, Cohen-Gonsaud M \& Henderson B (2006) Wake up! Peptidoglycan lysis and bacterial non-growth states. Trends in microbiology 14: 271-276.

Kerridge D, Horne RW \& Glauert AM (1962) Structural components of flagella from Salmonella typhimurium. Journal of molecular biology 4: 227-238.

Keseler IM, Bonavides-Martinez C, Collado-Vides J, et al. (2009) EcoCyc: a comprehensive view of Escherichia coli biology. Nucleic acids research 37: D464-470.

Kessler E (1995) beta-lytic endopeptidases. Methods in enzymology 248: 740-756.

Kessler E, Safrin M, Olson JC \& Ohman DE (1993) Secreted LasA of Pseudomonas aeruginosa is a staphylolytic protease. The Journal of biological chemistry 268: 75037508.

Kharazmi A (1989) Interactions of Pseudomonas aeruginosa proteases with the cells of the immune system. Antibiotics and chemotherapy 42: 42-49.

Kiehn TE (1989) Bacteremia and fungemia in the immunocompromised patient. European journal of clinical microbiology \& infectious diseases : official publication of the European Society of Clinical Microbiology 8: 832-837.

Kielhofner M, Atmar RL, Hamill RJ \& Musher DM (1992) Life-threatening Pseudomonas aeruginosa infections in patients with human immunodeficiency virus infection. Clinical infectious diseases : an official publication of the Infectious Diseases Society of America 14: 403-411.

King JD, Kocincova D, Westman EL \& Lam JS (2009) Review: Lipopolysaccharide biosynthesis in Pseudomonas aeruginosa. Innate immunity 15: 261-312.

Kipnis E, Sawa T \& Wiener-Kronish J (2006) Targeting mechanisms of Pseudomonas aeruginosa pathogenesis. Medecine et maladies infectieuses 36: 78-91.

Kitao T, Miyoshi-Akiyama T \& Kirikae T (2009) AAC(6')-laf, a novel aminoglycoside 6'-Nacetyltransferase from multidrug-resistant Pseudomonas aeruginosa clinical isolates. Antimicrobial agents and chemotherapy 53: 2327-2334. 
Klockgether J \& Tummler B (2017) Recent advances in understanding Pseudomonas aeruginosa as a pathogen. F1000Research 6: 1261.

Klockgether J, Cramer N, Wiehlmann L, Davenport CF \& Tummler B (2011) Pseudomonas aeruginosa genomic structure and diversity. Frontiers in microbiology 2: 150.

Koch AK, Kappeli O, Fiechter A \& Reiser J (1991) Hydrocarbon assimilation and biosurfactant production in Pseudomonas aeruginosa mutants. Journal of bacteriology 173: $4212-4219$.

Koch C \& Hoiby N (1993) Pathogenesis of cystic fibrosis. Lancet 341: 1065-1069.

Koh AY, Priebe GP \& Pier GB (2005) Virulence of Pseudomonas aeruginosa in a murine model of gastrointestinal colonization and dissemination in neutropenia. Infection and immunity 73: 2262-2272.

Kohanski MA, Dwyer DJ \& Collins JJ (2010) How antibiotics kill bacteria: from targets to networks. Nature reviews. Microbiology 8: 423-435.

Kohler T, Epp SF, Curty LK \& Pechere JC (1999) Characterization of MexT, the regulator of the MexE-MexF-OprN multidrug efflux system of Pseudomonas aeruginosa. Journal of bacteriology 181: 6300-6305.

Kohler T, Curty LK, Barja F, van Delden C \& Pechere JC (2000) Swarming of Pseudomonas aeruginosa is dependent on cell-to-cell signaling and requires flagella and pili. Journal of bacteriology 182: 5990-5996.

Kohler T, Michea-Hamzehpour M, Henze U, Gotoh N, Curty LK \& Pechere JC (1997) Characterization of MexE-MexF-OprN, a positively regulated multidrug efflux system of Pseudomonas aeruginosa. Molecular microbiology 23: 345-354.

Kohler T, Kok M, Michea-Hamzehpour M, et al. (1996) Multidrug efflux in intrinsic resistance to trimethoprim and sulfamethoxazole in Pseudomonas aeruginosa. Antimicrobial agents and chemotherapy 40: 2288-2290.

Kong F, Young L, Chen Y, et al. (2006) Pseudomonas aeruginosa pyocyanin inactivates lung epithelial vacuolar ATPase-dependent cystic fibrosis transmembrane conductance regulator expression and localization. Cellular microbiology 8: 1121-1133.

Kong KF, Schneper L \& Mathee K (2010) Beta-lactam antibiotics: from antibiosis to resistance and bacteriology. APMIS : acta pathologica, microbiologica, et immunologica Scandinavica 118: 1-36.

Kong KF, Aguila A, Schneper L \& Mathee K (2010) Pseudomonas aeruginosa betalactamase induction requires two permeases, AmpG and AmpP. BMC microbiology 10: 328.

Kong KF, Jayawardena SR, Del Puerto A, Wiehlmann L, Laabs U, Tummler B \& Mathee $\mathrm{K}$ (2005) Characterization of poxB, a chromosomal-encoded Pseudomonas aeruginosa oxacillinase. Gene 358: 82-92.

Kong KF, Jayawardena SR, Indulkar SD, Del Puerto A, Koh CL, Hoiby N \& Mathee K (2005) Pseudomonas aeruginosa AmpR is a global transcriptional factor that regulates expression of AmpC and PoxB beta-lactamases, proteases, quorum sensing, and other virulence factors. Antimicrobial agents and chemotherapy 49: 4567-4575. 
Koraimann G (2003) Lytic transglycosylases in macromolecular transport systems of Gram-negative bacteria. Cellular and molecular life sciences : CMLS 60: 2371-2388.

Korat B, Mottl H \& Keck W (1991) Penicillin-binding protein 4 of Escherichia coli: molecular cloning of the $d a c B$ gene, controlled overexpression, and alterations in murein composition. Molecular microbiology 5: 675-684.

Korfmann G \& Wiedemann B (1988) Genetic control of beta-lactamase production in Enterobacter cloacae. Reviews of infectious diseases 10: 793-799.

Korfmann G \& Sanders CC (1989) ampG is essential for high-level expression of AmpC beta-lactamase in Enterobacter cloacae. Antimicrobial agents and chemotherapy 33: 1946-1951.

Korgaonkar AK \& Whiteley M (2011) Pseudomonas aeruginosa enhances production of an antimicrobial in response to $\mathrm{N}$-acetylglucosamine and peptidoglycan. Journal of bacteriology 193: 909-917.

Korza HJ \& Bochtler M (2005) Pseudomonas aeruginosa LD-carboxypeptidase, a serine peptidase with a Ser-His-Glu triad and a nucleophilic elbow. The Journal of biological chemistry 280: 40802-40812.

Kotra LP, Haddad J \& Mobashery S (2000) Aminoglycosides: perspectives on mechanisms of action and resistance and strategies to counter resistance. Antimicrobial agents and chemotherapy 44: 3249-3256.

Kraft AR, Templin MF \& Holtje JV (1998) Membrane-bound lytic endotransglycosylase in Escherichia coli. Journal of bacteriology 180: 3441-3447.

Kraft AR, Prabhu J, Ursinus A \& Holtje JV (1999) Interference with murein turnover has no effect on growth but reduces beta-lactamase induction in Escherichia coli. Journal of bacteriology 181: 7192-7198.

Kumari H, Balasubramanian D, Zincke D \& Mathee K (2014) Role of Pseudomonas aeruginosa AmpR on beta-lactam and non-beta-lactam transient cross-resistance upon pre-exposure to subinhibitory concentrations of antibiotics. Journal of medical microbiology 63: 544-555.

Kumari H, Murugapiran SK, Balasubramanian D, et al. (2014) LTQ-XL mass spectrometry proteome analysis expands the Pseudomonas aeruginosa AmpR regulon to include cyclic di-GMP phosphodiesterases and phosphoproteins, and identifies novel open reading frames. Journal of proteomics 96: 328-342.

Kurachi M (1958) Studies on the Biosynthesis of Pyocyanine. (II) : Isolation and Determination of Pyocyanine. Vol. 36 ed.^eds.), p.^pp. 174-187. Bulletin of the Institute for Chemical Research, Kyoto.

Kusar D, Srimpf K, Isakovic $\mathrm{P}$, et al. (2016) Determination of $\mathrm{N}$-acylhomoserine lactones of Pseudomonas aeruginosa in clinical samples from dogs with otitis externa. BMC veterinary research 12: 233.

Kwon JH, Belanger JM, Sigouin M, Lanthier J, Willemot C \& Pare JJ (1990) Chemical constituents of Panax ginseng exposed to. gamma. irradiation. Journal of Agricultural and Food Chemistry 38: 830-834. 
Lamas Ferreiro JL, Alvarez Otero J, Gonzalez Gonzalez L, et al. (2017) Pseudomonas aeruginosa urinary tract infections in hospitalized patients: Mortality and prognostic factors. PloS one 12: e0178178.

Lambert PA (2002) Mechanisms of antibiotic resistance in Pseudomonas aeruginosa. Journal of the Royal Society of Medicine 95 Suppl 41: 22-26.

Lamers RP, Nguyen UT, Nguyen Y, Buensuceso RN \& Burrows LL (2015) Loss of membrane-bound lytic transglycosylases increases outer membrane permeability and beta-lactam sensitivity in Pseudomonas aeruginosa. MicrobiologyOpen 4: 879-895.

Lang AB, Horn MP, Imboden MA \& Zuercher AW (2004) Prophylaxis and therapy of Pseudomonas aeruginosa infection in cystic fibrosis and immunocompromised patients. Vaccine 22 Suppl 1: S44-48.

Langaee TY, Gagnon L \& Huletsky A (2000) Inactivation of the $a m p D$ gene in Pseudomonas aeruginosa leads to moderate-basal-level and hyperinducible AmpC beta-lactamase expression. Antimicrobial agents and chemotherapy 44: 583-589.

Langan KM, Kotsimbos T \& Peleg AY (2015) Managing Pseudomonas aeruginosa respiratory infections in cystic fibrosis. Current opinion in infectious diseases 28: 547556.

Larsen MW, Moser C, Hoiby N, Song Z \& Kharazmi A (2004) Ginseng modulates the immune response by induction of interleukin-12 production. APMIS : acta pathologica, microbiologica, et immunologica Scandinavica 112: 369-373.

Latifi A, Winson MK, Foglino M, Bycroft BW, Stewart GS, Lazdunski A \& Williams P (1995) Multiple homologues of LuxR and LuxI control expression of virulence determinants and secondary metabolites through quorum sensing in Pseudomonas aeruginosa PAO1. Molecular microbiology 17: 333-343.

Lauretti L, Riccio ML, Mazzariol A, Cornaglia G, Amicosante G, Fontana R \& Rossolini GM (1999) Cloning and characterization of blaviM, a new integron-borne metallo-betalactamase gene from a Pseudomonas aeruginosa clinical isolate. Antimicrobial agents and chemotherapy 43: 1584-1590.

Leclercq S, Derouaux A, Olatunji S, et al. (2017) Interplay between penicillin-binding proteins and SEDS proteins promotes bacterial cell wall synthesis. Scientific reports 7: 43306.

Lee DG, Urbach JM, Wu G, et al. (2006) Genomic analysis reveals that Pseudomonas aeruginosa virulence is combinatorial. Genome biology 7: R90.

Lee EJ, Evans DJ \& Fleiszig SM (2003) Role of Pseudomonas aeruginosa ExsA in penetration through corneal epithelium in a novel in vivo model. Investigative ophthalmology \& visual science 44: 5220-5227.

Lee J, Wu J, Deng Y, et al. (2013) A cell-cell communication signal integrates quorum sensing and stress response. Nature chemical biology 9: 339-343.

Lee M, Zhang W, Hesek D, Noll BC, Boggess B \& Mobashery S (2009) Bacterial AmpD at the crossroads of peptidoglycan recycling and manifestation of antibiotic resistance. Journal of the American Chemical Society 131: 8742-8743. 
Lee M, Hesek D, Shah IM, Oliver AG, Dworkin J \& Mobashery S (2010) Synthetic peptidoglycan motifs for germination of bacterial spores. Chembiochem : a European journal of chemical biology 11: 2525-2529.

Lee M, Hesek D, Llarrull LI, Lastochkin E, Pi H, Boggess B \& Mobashery S (2013) Reactions of all Escherichia coli lytic transglycosylases with bacterial cell wall. Journal of the American Chemical Society 135: 3311-3314.

Lee M, Hesek D, Blazquez B, Lastochkin E, Boggess B, Fisher JF \& Mobashery S (2015) Catalytic spectrum of the penicillin-binding protein 4 of Pseudomonas aeruginosa, a nexus for the induction of beta-lactam antibiotic resistance. Journal of the American Chemical Society 137: 190-200.

Lee M, Dominguez-Gil T, Hesek D, Mahasenan KV, Lastochkin E, Hermoso JA \& Mobashery S (2016) Turnover of bacterial cell wall by SItB3, a multidomain lytic transglycosylase of Pseudomonas aeruginosa. ACS chemical biology.

Lee M, Dhar S, De Benedetti S, et al. (2016) Muropeptides in Pseudomonas aeruginosa and their role as elicitors of beta-lactam-antibiotic resistance. Angewandte Chemie.

Lee M, Dhar S, De Benedetti S, et al. (2016) Corrigendum: Muropeptides in Pseudomonas aeruginosa and their role as elicitors of beta-lactam-antibiotic resistance. Angewandte Chemie 55: 12568.

Lee M, Hesek D, Dik DA, et al. (2017) From genome to proteome to elucidation of reactions for all eleven known lytic transglycosylases from Pseudomonas aeruginosa. Angewandte Chemie 56: 2735-2739.

Lee M, Artola-Recolons C, Carrasco-Lopez C, et al. (2013) Cell-wall remodeling by the zinc-protease AmpDh3 from Pseudomonas aeruginosa. Journal of the American Chemical Society 135: 12604-12607.

Legaree BA, Daniels K, Weadge JT, Cockburn D \& Clarke AJ (2007) Function of penicillin-binding protein 2 in viability and morphology of Pseudomonas aeruginosa. The Journal of antimicrobial chemotherapy 59: 411-424.

Legnani P, Leoni E, Rapuano S, Turin D \& Valenti C (1999) Survival and growth of Pseudomonas aeruginosa in natural mineral water: a 5-year study. International journal of food microbiology 53: 153-158.

Leung KW \& Wong AS (2010) Pharmacology of ginsenosides: a literature review. Chinese medicine 5: 20.

Li H, Luo YF, Williams BJ, Blackwell TS \& Xie CM (2012) Structure and function of OprD protein in Pseudomonas aeruginosa: from antibiotic resistance to novel therapies. International journal of medical microbiology : IJMM 302: 63-68.

Li P, Ying J, Yang G, et al. (2016) Structure-function analysis of the transmembrane protein AmpG from Pseudomonas aeruginosa. PloS one 11: e0168060.

Li XZ, Nikaido H \& Poole K (1995) Role of mexA-mexB-oprM in antibiotic efflux in Pseudomonas aeruginosa. Antimicrobial agents and chemotherapy 39: 1948-1953.

Li Y, Mima T, Komori Y, Morita Y, Kuroda T, Mizushima T \& Tsuchiya T (2003) A new member of the tripartite multidrug efflux pumps, MexVW-OprM, in Pseudomonas aeruginosa. The Journal of antimicrobial chemotherapy 52: 572-575. 
Li Z, Clarke AJ \& Beveridge TJ (1996) A major autolysin of Pseudomonas aeruginosa: subcellular distribution, potential role in cell growth and division and secretion in surface membrane vesicles. Journal of bacteriology 178: 2479-2488.

Liao X \& Hancock RE (1997) Identification of a penicillin-binding protein 3 homolog, PBP3x, in Pseudomonas aeruginosa: gene cloning and growth phase-dependent expression. Journal of bacteriology 179: 1490-1496.

Lindberg F \& Normark S (1987) Common mechanism of $a m p C$ beta-lactamase induction in enterobacteria: regulation of the cloned Enterobacter cloacae P99 beta-lactamase gene. Journal of bacteriology 169: 758-763.

Lindquist S, Galleni M, Lindberg F \& Normark S (1989) Signalling proteins in enterobacterial AmpC beta-lactamase regulation. Molecular microbiology 3: 1091-1102.

Lindquist S, Weston-Hafer K, Schmidt H, et al. (1993) AmpG, a signal transducer in chromosomal beta-lactamase induction. Molecular microbiology 9: 703-715.

Lister PD, Wolter DJ \& Hanson ND (2009) Antibacterial-resistant Pseudomonas aeruginosa: clinical impact and complex regulation of chromosomally encoded resistance mechanisms. Clinical microbiology reviews 22: 582-610.

Livermore DM (1995) beta-Lactamases in laboratory and clinical resistance. Clinical microbiology reviews 8: 557-584.

Livermore DM (2002) Multiple mechanisms of antimicrobial resistance in Pseudomonas aeruginosa: our worst nightmare? Clinical infectious diseases : an official publication of the Infectious Diseases Society of America 34: 634-640.

Lodge J, Busby S \& Piddock L (1993) Investigation of the Pseudomonas aeruginosa ampR gene and its role at the chromosomal ampC beta-lactamase promoter. FEMS microbiology letters 111: 315-320.

Lodge JM, Minchin SD, Piddock LJ \& Busby SJ (1990) Cloning, sequencing and analysis of the structural gene and regulatory region of the Pseudomonas aeruginosa chromosomal ampC beta-lactamase. The Biochemical journal 272: 627-631.

Lommatzsch J, Templin MF, Kraft AR, Vollmer W \& Holtje JV (1997) Outer membrane localization of murein hydrolases: MltA, a third lipoprotein lytic transglycosylase in Escherichia coli. Journal of bacteriology 179: 5465-5470.

Lopez-Vallejo F, Giulianotti MA, Houghten RA \& Medina-Franco JL (2012) Expanding the medicinally relevant chemical space with compound libraries. Drug discovery today 17: 718-726.

Lovering AM, White LO \& Reeves DS (1987) AAC(1): a new aminoglycoside-acetylating enzyme modifying the $\mathrm{Cl}$ aminogroup of apramycin. The Journal of antimicrobial chemotherapy 20: 803-813.

Luker KE, Collier JL, Kolodziej EW, Marshall GR \& Goldman WE (1993) Bordetella pertussis tracheal cytotoxin and other muramyl peptides: distinct structure-activity relationships for respiratory epithelial cytopathology. Proceedings of the National Academy of Sciences of the United States of America 90: 2365-2369.

Lyczak JB, Cannon CL \& Pier GB (2000) Establishment of Pseudomonas aeruginosa infection: lessons from a versatile opportunist. Microbes and infection / Institut Pasteur 2: 1051-1060. 
Lyczak JB, Cannon CL \& Pier GB (2000) Establishment of Pseudomonas aeruginosa infection: lessons from a versatile opportunist. Microbes and infection 2: 1051-1060.

Lyczak JB, Cannon CL \& Pier GB (2002) Lung infections associated with cystic fibrosis. Clinical microbiology reviews 15: 194-222.

Lynch SV \& Wiener-Kronish JP (2008) Novel strategies to combat bacterial virulence. Current opinion in critical care 14: 593-599.

Lysenko O (1961) Pseudomonas--an attempt at a general classification. Journal of general microbiology 25: 379-408.

Ma L, Wang S, Wang D, Parsek MR \& Wozniak DJ (2012) The roles of biofilm matrix polysaccharide $\mathrm{Psl}$ in mucoid Pseudomonas aeruginosa biofilms. FEMS immunology and medical microbiology 65: 377-380.

Makemson J, Eberhard A \& Mathee K (2006) Simple electrospray mass spectrometry detection of acylhomoserine lactones. Luminescence : the journal of biological and chemical luminescence 21: 1-6.

Marchler-Bauer A, Bo Y, Han L, et al. (2017) CDD/SPARCLE: functional classification of proteins via subfamily domain architectures. Nucleic acids research 45: D200-D203.

Marks MI (1981) The pathogenesis and treatment of pulmonary infections in patients with cystic fibrosis. The Journal of pediatrics 98: 173-179.

Marks Ml (1990) Clinical significance of Staphylococcus aureus in cystic fibrosis. Infection 18: 53-56.

Marshall B, Faro A, Elbert A, et al. (2016) Cystic Fibrosis Foundation Patient Registry. ed. ${ }^{\wedge}$ eds.), p. ${ }^{\wedge}$ pp.

Martinez-Caballero S, Lee M, Artola-Recolons C, et al. (2013) Reaction products and the X-ray structure of AmpDh2, a virulence determinant of Pseudomonas aeruginosa. Journal of the American Chemical Society 135: 10318-10321.

Martinez-Solano L, Macia MD, Fajardo A, Oliver A \& Martinez JL (2008) Chronic Pseudomonas aeruginosa infection in chronic obstructive pulmonary disease. Clinical infectious diseases : an official publication of the Infectious Diseases Society of America 47: 1526-1533.

Marvig RL, Sommer LM, Molin S \& Johansen HK (2015) Convergent evolution and adaptation of Pseudomonas aeruginosa within patients with cystic fibrosis. Nature genetics 47: 57-64.

Mathee K, Ciofu O, Sternberg C, et al. (1999) Mucoid conversion of Pseudomonas aeruginosa by hydrogen peroxide: a mechanism for virulence activation in the cystic fibrosis lung. Microbiology 145 ( Pt 6): 1349-1357.

Mathee K, Narasimhan G, Valdes C, et al. (2008) Dynamics of Pseudomonas aeruginosa genome evolution. Proceedings of the National Academy of Sciences of the United States of America 105: 3100-3105.

Matias VR \& Beveridge TJ (2007) Cryo-electron microscopy of cell division in Staphylococcus aureus reveals a mid-zone between nascent cross walls. Molecular microbiology 64: 195-206. 
Matias VR, Al-Amoudi A, Dubochet J \& Beveridge TJ (2003) Cryo-transmission electron microscopy of frozen-hydrated sections of Escherichia coli and Pseudomonas aeruginosa. Journal of bacteriology 185: 6112-6118.

Matsuhashi M, Tamaki S, Curtis SJ \& Strominger JL (1979) Mutational evidence for identity of penicillin-binding protein 5 in Escherichia coli with the major D-alanine carboxypeptidase IA activity. Journal of bacteriology 137: 644-647.

Matthew M (1979) Plasmid-mediated beta-lactamases of Gram-negative bacteria: properties and distribution. The Journal of antimicrobial chemotherapy 5: 349-358.

Matthew M, Hedges RW \& Smith JT (1979) Types of beta-lactamase determined by plasmids in Gram-negative bacteria. Journal of bacteriology 138: 657-662.

Mauck J, Chan L \& Glaser L (1971) Turnover of the cell wall of Gram-positive bacteria. The Journal of biological chemistry 246: 1820-1827.

Mavrodi DV, Bonsall RF, Delaney SM, Soule MJ, Phillips G \& Thomashow LS (2001) Functional analysis of genes for biosynthesis of pyocyanin and phenazine-1carboxamide from Pseudomonas aeruginosa PAO1. Journal of bacteriology 183: 64546465.

McDonald C, Inohara N \& Nunez G (2005) Peptidoglycan signaling in innate immunity and inflammatory disease. The Journal of biological chemistry 280: 20177-20180.

McManus AT, Mason AD, Jr., McManus WF \& Pruitt BA, Jr. (1985) Twenty-five year review of Pseudomonas aeruginosa bacteremia in a burn center. European journal of clinical microbiology 4: 219-223.

McMorran B, Town L, Costelloe E, Palmer J, Engel J, Hume D \& Wainwright B (2003) Effector ExoU from the type III secretion system is an important modulator of gene expression in lung epithelial cells in response to Pseudomonas aeruginosa infection. Infection and immunity 71: 6035-6044.

Meberg BM, Paulson AL, Priyadarshini R \& Young KD (2004) Endopeptidase penicillinbinding proteins 4 and 7 play auxiliary roles in determining uniform morphology of Escherichia coli. Journal of bacteriology 186: 8326-8336.

Medeiros AA (1997) Evolution and dissemination of beta-lactamases accelerated by generations of beta-lactam antibiotics. Clinical infectious diseases : an official publication of the Infectious Diseases Society of America 24 Suppl 1: S19-45.

Meeske AJ, Sham LT, Kimsey H, Koo BM, Gross CA, Bernhardt TG \& Rudner DZ (2015) MurJ and a novel lipid II flippase are required for cell wall biogenesis in Bacillus subtilis. Proceedings of the National Academy of Sciences of the United States of America 112: 6437-6442.

Melvin P. Weinstein M (2018) Performance standards for antimicrobial susceptibility testing. ed.^eds.), p.^pp. 296.

Mena KD \& Gerba CP (2009) Risk assessment of Pseudomonas aeruginosa in water. Reviews of environmental contamination and toxicology 201: 71-115.

Mengin-Lecreulx D \& van Heijenoort J (1994) Copurification of glucosamine-1phosphate acetyltransferase and $\mathrm{N}$-acetylglucosamine-1-phosphate uridyltransferase activities of Escherichia coli: characterization of the $g / m U$ gene product as a bifunctional 
enzyme catalyzing two subsequent steps in the pathway for UDP-N-acetylglucosamine synthesis. Journal of bacteriology 176: $5788-5795$.

Mengin-Lecreulx D \& van Heijenoort J (1996) Characterization of the essential gene glmM encoding phosphoglucosamine mutase in Escherichia coli. The Journal of biological chemistry 271: 32-39.

Mengin-Lecreulx D, van Heijenoort J \& Park JT (1996) Identification of the $\mathrm{mpl}$ gene encoding UDP-N-acetylmuramate: L-alanyl-gamma-D-glutamyl-meso-diaminopimelate ligase in Escherichia coli and its role in recycling of cell wall peptidoglycan. Journal of bacteriology 178: 5347-5352.

Mengin-Lecreulx D, Texier L, Rousseau M \& van Heijenoort J (1991) The murG gene of Escherichia coli codes for the UDP-N-acetylglucosamine: $\mathrm{N}$-acetylmuramyl(pentapeptide) pyrophosphoryl-undecaprenol $\mathrm{N}$-acetylglucosamine transferase involved in the membrane steps of peptidoglycan synthesis. Journal of bacteriology 173: 46254636.

Mercier F, Zervosen A, Teller N, et al. (2010) 1,6-AnhMurNAc derivatives for assay development of amidase AmiD. Bioorganic \& medicinal chemistry 18: 7422-7431.

Miall LS, McGinley NT, Brownlee KG \& Conway SP (2001) Methicillin resistant Staphylococcus aureus (MRSA) infection in cystic fibrosis. Archives of disease in childhood 84: 160-162.

Michel L, Bachelard A \& Reimmann C (2007) Ferripyochelin uptake genes are involved in pyochelin-mediated signalling in Pseudomonas aeruginosa. Microbiology 153: 15081518.

Mielcarek N, Debrie AS, Raze D, et al. (2006) Live attenuated B. pertussis as a singledose nasal vaccine against whooping cough. PLoS pathogens 2: e65.

Migiyama Y, Yanagihara K, Kaku N, et al. (2016) Pseudomonas aeruginosa bacteremia among immunocompetent and immunocompromised patients: relation to initial antibiotic therapy and survival. Japanese journal of infectious diseases 69: 91-96.

Miller MB \& Bassler BL (2001) Quorum sensing in bacteria. Annual Reviews in Microbiology 55: 165-199.

Mima T, Joshi S, Gomez-Escalada M \& Schweizer HP (2007) Identification and characterization of TriABC-OpmH, a triclosan efflux pump of Pseudomonas aeruginosa requiring two membrane fusion proteins. Journal of bacteriology 189: 7600-7609.

Mima T, Sekiya H, Mizushima T, Kuroda T \& Tsuchiya T (2005) Gene cloning and properties of the RND-type multidrug efflux pumps MexPQ-OpmE and MexMN-OprM from Pseudomonas aeruginosa. Microbiology and immunology 49: 999-1002.

Mima T, Kohira N, Li Y, Sekiya H, Ogawa W, Kuroda T \& Tsuchiya T (2009) Gene cloning and characteristics of the RND-type multidrug efflux pump MuxABC-OpmB possessing two RND components in Pseudomonas aeruginosa. Microbiology 155: 35093517.

Mine T, Morita Y, Kataoka A, Mizushima T \& Tsuchiya T (1999) Expression in Escherichia coli of a new multidrug efflux pump, MexXY, from Pseudomonas aeruginosa. Antimicrobial agents and chemotherapy 43: 415-417. 
Mitsuhashi S IM (1981) Mechanisms of resistance to beta-Lactam antibiotics. In S. Mitsuhashi (ed.), Beta-lactam antibiotics p. 41-56.

Mohammadi T, van Dam V, Sijbrandi R, et al. (2011) Identification of FtsW as a transporter of lipid-linked cell wall precursors across the membrane. The EMBO journal 30: $1425-1432$.

Mondon M, Hur S, Vadlamani G, et al. (2013) Selective trihydroxyazepane NagZ inhibitors increase sensitivity of Pseudomonas aeruginosa to beta-lactams. Chemical communications 49: 10983-10985.

Moore RA, DeShazer D, Reckseidler S, Weissman A \& Woods DE (1999) Effluxmediated aminoglycoside and macrolide resistance in Burkholderia pseudomallei. Antimicrobial agents and chemotherapy 43: 465-470.

Morita Y, Tomida J \& Kawamura Y (2012) MexXY multidrug efflux system of Pseudomonas aeruginosa. Frontiers in microbiology 3: 408.

Moya B, Juan C, Alberti S, Perez JL \& Oliver A (2008) Benefit of having multiple ampD genes for acquiring beta-lactam resistance without losing fitness and virulence in Pseudomonas aeruginosa. Antimicrobial agents and chemotherapy 52: 3694-3700.

Moya B, Dotsch A, Juan C, Blazquez J, Zamorano L, Haussler S \& Oliver A (2009) Betalactam resistance response triggered by inactivation of a nonessential penicillin-binding protein. PLoS pathogens 5: e1000353.

Moya B, Beceiro A, Cabot G, Juan C, Zamorano L, Alberti S \& Oliver A (2012) Pan-betalactam resistance development in Pseudomonas aeruginosa clinical strains: molecular mechanisms, penicillin-binding protein profiles, and binding affinities. Antimicrobial agents and chemotherapy 56: 4771-4778.

Mugnier P, Casin I, Bouthors AT \& Collatz E (1998) Novel OXA-10-derived extendedspectrum beta-lactamases selected in vivo or in vitro. Antimicrobial agents and chemotherapy 42: 3113-3116.

Mukamolova GV, Murzin AG, Salina EG, Demina GR, Kell DB, Kaprelyants AS \& Young M (2006) Muralytic activity of Micrococcus luteus Rpf and its relationship to physiological activity in promoting bacterial growth and resuscitation. Molecular microbiology 59: 8498.

Muramatsu H, Horii T, Morita M, Hashimoto H, Kanno T \& Maekawa M (2003) Effect of basic amino acids on susceptibility to carbapenems in clinical Pseudomonas aeruginosa isolates. International journal of medical microbiology : IJMM 293: 191-197.

Nakagawa J, Tamaki S, Tomioka S \& Matsuhashi M (1984) Functional biosynthesis of cell wall peptidoglycan by polymorphic bifunctional polypeptides. Penicillin-binding protein 1Bs of Escherichia coli with activities of transglycosylase and transpeptidase. The Journal of biological chemistry 259: 13937-13946.

Nakamura M, Maruyama IN, Soma M, Kato J, Suzuki H \& Horota Y (1983) On the process of cellular division in Escherichia coli: nucleotide sequence of the gene for penicillin-binding protein 3. Molecular \& general genetics : MGG 191: 1-9.

Nakamura S, Nakamura M, Kojima T \& Yoshida H (1989) gyrA and gyrB mutations in quinolone-resistant strains of Escherichia coli. Antimicrobial agents and chemotherapy 33: $254-255$. 
Nambu T, Minamino T, Macnab RM \& Kutsukake K (1999) Peptidoglycan-hydrolyzing activity of the FlgJ protein, essential for flagellar rod formation in Salmonella typhimurium. J Bacteriol 181: 1555-1561.

Nealson KH \& Hastings JW (1979) Bacterial bioluminescence: its control and ecological significance. Microbiological reviews 43: 496-518.

Nefzi A, Ostresh JM, Yu Y \& Houghten RA (2004) Combinatorial chemistry: libraries from libraries, the art of the diversity-oriented transformation of resin-bound peptides and chiral polyamides to low molecular weight acyclic and heterocyclic compounds. The Journal of organic chemistry 69: 3603-3609.

Nelson DE \& Young KD (2000) Penicillin binding protein 5 affects cell diameter, contour, and morphology of Escherichia coli. Journal of bacteriology 182: 1714-1721.

Nelson DE \& Young KD (2001) Contributions of PBP 5 and DD-carboxypeptidase penicillin binding proteins to maintenance of cell shape in Escherichia coli. Journal of bacteriology 183: 3055-3064.

Nguyen-Disteche M, Fraipont C, Buddelmeijer N \& Nanninga N (1998) The structure and function of Escherichia coli penicillin-binding protein 3. Cellular and molecular life sciences : CMLS 54: 309-316.

Nicas TI \& Hancock RE (1983) Pseudomonas aeruginosa outer membrane permeability: isolation of a porin protein F-deficient mutant. Journal of bacteriology 153: 281-285.

Nikaido H, Nikaido K \& Harayama S (1991) Identification and characterization of porins in Pseudomonas aeruginosa. The Journal of biological chemistry 266: 770-779.

Nishino N \& Powers JC (1980) Pseudomonas aeruginosa elastase. Development of a new substrate, inhibitors, and an affinity ligand. The Journal of biological chemistry 255: 3482-3486.

Noguchi H, Matsuhashi M \& Mitsuhashi S (1979) Comparative studies of penicillinbinding proteins in Pseudomonas aeruginosa and Escherichia coli. European journal of biochemistry / FEBS 100: 41-49.

Noguchi H, Fukasawa M, Komatsu T, Mitsuhashi S \& Matsuhashi M (1985) Mutation in Pseudomonas aeruginosa causing simultaneous defects in penicillin-binding protein 5 and in enzyme activities of penicillin release and D-alanine carboxypeptidase. Journal of bacteriology 162: 849-851.

Nordmann P, Ronco E, Naas T, Duport C, Michel-Briand Y \& Labia R (1993) Characterization of a novel extended-spectrum beta-lactamase from Pseudomonas aeruginosa. Antimicrobial agents and chemotherapy 37: 962-969.

Normark S (1995) beta-Lactamase induction in Gram-negative bacteria is intimately linked to peptidoglycan recycling. Microbial drug resistance 1: 111-114.

Normark S \& Lindberg F (1985) Evolution of resistance to penicillin and cephalosporin antibiotics. BioEssays : news and reviews in molecular, cellular and developmental biology 3: 22-26.

Nossal NG \& Heppel LA (1966) The release of enzymes by osmotic shock from Escherichia coli in exponential phase. The Journal of biological chemistry 241: 30553062. 
O'Callaghan $\mathrm{CH}$, Morris A, Kirby SM \& Shingler AH (1972) Novel method for detection of beta-lactamases by using a chromogenic cephalosporin substrate. Antimicrobial agents and chemotherapy 1: 283-288.

O'Loughlin CT, Miller LC, Siryaporn A, Drescher K, Semmelhack MF \& Bassler BL (2013) A quorum-sensing inhibitor blocks Pseudomonas aeruginosa virulence and biofilm formation. Proceedings of the National Academy of Sciences of the United States of America 110: 17981-17986.

O'Toole GA (2011) Microtiter dish biofilm formation assay. Journal of visualized experiments : JoVE.

O'Toole GA \& Kolter R (1998) Flagellar and twitching motility are necessary for Pseudomonas aeruginosa biofilm development. Molecular microbiology 30: 295-304.

Ochs MM, Lu CD, Hancock RE \& Abdelal AT (1999) Amino acid-mediated induction of the basic amino acid-specific outer membrane porin OprD from Pseudomonas aeruginosa. Journal of bacteriology 181: 5426-5432.

Ochsner UA, Fiechter A \& Reiser J (1994) Isolation, characterization, and expression in Escherichia coli of the Pseudomonas aeruginosa rhIAB genes encoding a rhamnosyltransferase involved in rhamnolipid biosurfactant synthesis. The Journal of biological chemistry 269: 19787-19795.

Ochsner UA, Wilderman PJ, Vasil AI \& Vasil ML (2002) GeneChip expression analysis of the iron starvation response in Pseudomonas aeruginosa: identification of novel pyoverdine biosynthesis genes. Molecular microbiology 45: 1277-1287.

Oh H, Stenhoff J, Jalal S \& Wretlind B (2003) Role of efflux pumps and mutations in genes for topoisomerases II and IV in fluoroquinolone-resistant Pseudomonas aeruginosa strains. Microbial drug resistance 9: 323-328.

Ohman DE, Cryz SJ \& Iglewski BH (1980) Isolation and characterization of Pseudomonas aeruginosa PAO mutant that produces altered elastase. Journal of bacteriology 142: 836-842.

Okazaki A \& Avison MB (2008) Induction of L1 and L2 beta-lactamase production in Stenotrophomonas maltophilia is dependent on an AmpR-type regulator. Antimicrobial agents and chemotherapy 52: 1525-1528.

Oldenburg KR, Vo KT, Michaelis S \& Paddon C (1997) Recombination-mediated PCRdirected plasmid construction in vivo in yeast. Nucleic Acids Res 25: 451-452.

Olsen RH, DeBusscher G \& McCombie WR (1982) Development of broad-host-range vectors and gene banks: self-cloning of the Pseudomonas aeruginosa PAO chromosome. Journal of bacteriology 150: 60-69.

Osmon S, Ward S, Fraser VJ \& Kollef MH (2004) Hospital mortality for patients with bacteremia due to Staphylococcus aureus or Pseudomonas aeruginosa. Chest 125: 607-616.

Page B, Page M \& Noel C (1993) A new fluorometric assay for cytotoxicity measurements in-vitro. International journal of oncology 3: 473-476.

Palleroni N (2001) Pseudomonadaceae. ed.^eds.), p.^pp. Baltimore, MD. 
Palleroni NJ (2010) The Pseudomonas story. Environmental microbiology 12: 13771383.

Palleroni NJaM, R.B.E. (2004) Pseudomonas:Genomics, lifestyle and molecular architecture. (Ramos J-L, ed.^eds.), p.^pp. Springer US.

Palzkill T (2013) Metallo-beta-lactamase structure and function. Annals of the New York Academy of Sciences 1277: 91-104.

Panda SK, Mohanta YK, Padhi L, Park YH, Mohanta TK \& Bae H (2016) Large scale screening of ethnomedicinal plants for identification of potential antibacterial compounds. Molecules 21: 293.

Papanicolaou GA, Medeiros AA \& Jacoby GA (1990) Novel plasmid-mediated betalactamase (MIR-1) conferring resistance to oxyimino- and alpha-methoxy beta-lactams in clinical isolates of Klebsiella pneumoniae. Antimicrobial agents and chemotherapy 34: 2200-2209.

Papenfort K \& Bassler BL (2016) Quorum sensing signal-response systems in Gramnegative bacteria. Nature reviews. Microbiology 14: 576-588.

Park H, Cho B-G \& Lee M-K (1990) Nitrogen compounds of Korea ginseng and their physiological significance. Journal of Ginseng Research 14: 317-331.

Park JT (1993) Turnover and recycling of the murein sacculus in oligopeptide permeasenegative strains of Escherichia coli: indirect evidence for an alternative permease system and for a monolayered sacculus. Journal of bacteriology 175: 7-11.

Park JT (2001) Identification of a dedicated recycling pathway for anhydro-Nacetylmuramic acid and $\mathrm{N}$-acetylglucosamine derived from Escherichia coli cell wall murein. Journal of bacteriology 183: 3842-3847.

Park JT \& Burman LG (1985) Elongation of the murein sacculus of Escherichia coli. Annales de l'Institut Pasteur. Microbiologie 136A: 51-58.

Park JT \& Uehara T (2008) How bacteria consume their own exoskeletons (turnover and recycling of cell wall peptidoglycan). Microbiology and molecular biology reviews : MMBR 72: 211-227, table of contents.

Parsek MR, Val DL, Hanzelka BL, Cronan JE, Jr. \& Greenberg EP (1999) Acyl homoserine-lactone quorum-sensing signal generation. Proceedings of the National Academy of Sciences of the United States of America 96: 4360-4365.

Paterson DL \& Bonomo RA (2005) Extended-spectrum beta-lactamases: a clinical update. Clinical microbiology reviews 18: 657-686.

Patriquin GM, Banin E, Gilmour C, Tuchman R, Greenberg EP \& Poole K (2008) Influence of quorum sensing and iron on twitching motility and biofilm formation in Pseudomonas aeruginosa. Journal of bacteriology 190: 662-671.

Payne DJ, Cramp R, Winstanley DJ \& Knowles DJ (1994) Comparative activities of clavulanic acid, sulbactam, and tazobactam against clinically important beta-lactamases. Antimicrobial agents and chemotherapy 38: 767-772.

Pearson JP, Pesci EC \& Iglewski BH (1997) Roles of Pseudomonas aeruginosa las and rhl quorum-sensing systems in control of elastase and rhamnolipid biosynthesis genes. Journal of bacteriology 179: 5756-5767. 
Pearson JP, Passador L, Iglewski BH \& Greenberg EP (1995) A second Nacylhomoserine lactone signal produced by Pseudomonas aeruginosa. Proceedings of the National Academy of Sciences of the United States of America 92: 1490-1494.

Pedersen SS (1992) Lung infection with alginate-producing, mucoid Pseudomonas aeruginosa in cystic fibrosis. APMIS. Supplementum 28: 1-79.

Pedersen SS, Kharazmi A, Espersen F \& Hoiby N (1990) Pseudomonas aeruginosa alginate in cystic fibrosis sputum and the inflammatory response. Infection and immunity 58: 3363-3368.

Pepper JW (2008) Defeating pathogen drug resistance: guidance from evolutionary theory. Evolution; international journal of organic evolution 62: 3185-3191.

Pereira SG, Rosa AC, Ferreira AS, Moreira LM, Proenca DN, Morais PV \& Cardoso O (2014) Virulence factors and infection ability of Pseudomonas aeruginosa isolates from a hydropathic facility and respiratory infections. Journal of applied microbiology 116: 13591368.

Perkins HR (1963) Chemical structure and biosynthesis of bacterial cell walls. Bacteriological reviews 27: 18-55.

Perley-Robertson GE, Yadav AK, Winogrodzki JL, Stubbs KA, Mark BL \& Vocadlo DJ (2016) A fluorescent transport assay enables studying AmpG permeases involved in peptidoglycan recycling and antibiotic resistance. ACS chemical biology.

Perron K, Caille O, Rossier C, Van Delden C, Dumas JL \& Kohler T (2004) CzcR-CzcS, a two-component system involved in heavy metal and carbapenem resistance in Pseudomonas aeruginosa. The Journal of biological chemistry 279: 8761-8768.

Pesci EC, Pearson JP, Seed PC \& Iglewski BH (1997) Regulation of las and $\mathrm{rh} /$ quorum sensing in Pseudomonas aeruginosa. Journal of bacteriology 179: 3127-3132.

Pesci EC, Milbank JB, Pearson JP, McKnight S, Kende AS, Greenberg EP \& Iglewski $\mathrm{BH}$ (1999) Quinolone signaling in the cell-to-cell communication system of Pseudomonas aeruginosa. Proceedings of the National Academy of Sciences of the United States of America 96: 11229-11234.

Pier GB (2007) Pseudomonas aeruginosa lipopolysaccharide: a major virulence factor, initiator of inflammation and target for effective immunity. International journal of medical microbiology : IJMM 297: 277-295.

Pittet D, Harbarth S, Ruef C, et al. (1999) Prevalence and risk factors for nosocomial infections in four university hospitals in Switzerland. Infection control and hospital epidemiology 20: 37-42.

Plumbridge $J$ (1995) Co-ordinated regulation of amino sugar biosynthesis and degradation: the NagC repressor acts as both an activator and a repressor for the transcription of the gImUS operon and requires two separated NagC binding sites. The EMBO journal 14: 3958-3965.

Plumbridge $J$ (2009) An alternative route for recycling of $\mathrm{N}$-acetylglucosamine from peptidoglycan involves the $\mathrm{N}$-acetylglucosamine phosphotransferase system in Escherichia coli. Journal of bacteriology 191: 5641-5647.

Poirel L, Naas T \& Nordmann P (2010) Diversity, epidemiology, and genetics of class D beta-lactamases. Antimicrobial agents and chemotherapy 54:24-38. 
Poirel L, Le Thomas I, Naas T, Karim A \& Nordmann P (2000) Biochemical sequence analyses of GES-1, a novel class A extended-spectrum beta-lactamase, and the class 1 integron In52 from Klebsiella pneumoniae. Antimicrobial agents and chemotherapy 44: 622-632.

Poirel L, Naas T, Nicolas D, Collet L, Bellais S, Cavallo JD \& Nordmann P (2000) Characterization of VIM-2, a carbapenem-hydrolyzing metallo-beta-lactamase and its plasmid- and integron-borne gene from a Pseudomonas aeruginosa clinical isolate in France. Antimicrobial agents and chemotherapy 44: 891-897.

Pomorski T \& Menon AK (2006) Lipid flippases and their biological functions. Cellular and molecular life sciences : CMLS 63: 2908-2921.

Poole K (2000) Efflux-mediated resistance to fluoroquinolones in Gram-negative bacteria. Antimicrobial agents and chemotherapy 44: 2233-2241.

Poole K (2011) Pseudomonas aeruginosa: resistance to the max. Frontiers in microbiology 2: 65.

Poole K, Krebes K, McNally C \& Neshat S (1993) Multiple antibiotic resistance in Pseudomonas aeruginosa: evidence for involvement of an efflux operon. Journal of bacteriology 175: 7363-7372.

Poole K, Tetro K, Zhao Q, Neshat S, Heinrichs DE \& Bianco N (1996) Expression of the multidrug resistance operon mexA-mexB-oprM in Pseudomonas aeruginosa: mex $R$ encodes a regulator of operon expression. Antimicrobial agents and chemotherapy 40: 2021-2028.

Poole K, Gotoh N, Tsujimoto H, et al. (1996) Overexpression of the mexC-mexD-oprJ efflux operon in $n f x B$-type multidrug-resistant strains of Pseudomonas aeruginosa. Molecular microbiology 21: 713-724.

Potvin E, Lehoux DE, Kukavica-Ibrulj I, Richard KL, Sanschagrin F, Lau GW \& Levesque RC (2003) In vivo functional genomics of Pseudomonas aeruginosa for high-throughput screening of new virulence factors and antibacterial targets. Environmental microbiology 5: 1294-1308.

Preston MJ, Seed PC, Toder DS, et al. (1997) Contribution of proteases and LasR to the virulence of Pseudomonas aeruginosa during corneal infections. Infection and immunity 65: 3086-3090.

Priyadarshini R, de Pedro MA \& Young KD (2007) Role of peptidoglycan amidases in the development and morphology of the division septum in Escherichia coli. Journal of bacteriology 189: 5334-5347.

Proenca R, Niu WW, Cacalano G \& Prince A (1993) The Pseudomonas cepacia 249 chromosomal penicillinase is a member of the AmpC family of chromosomal betalactamases. Antimicrobial agents and chemotherapy 37: 667-674.

Qiao Y, Lebar MD, Schirner K, Schaefer K, Tsukamoto H, Kahne D \& Walker S (2014) Detection of lipid-linked peptidoglycan precursors by exploiting an unexpected transpeptidase reaction. Journal of the American Chemical Society 136: 14678-14681.

Quintela JC, Caparros M \& de Pedro MA (1995) Variability of peptidoglycan structural parameters in Gram-negative bacteria. FEMS microbiology letters 125: 95-100. 
Ramirez MS \& Tolmasky ME (2010) Aminoglycoside modifying enzymes. Drug resistance updates : reviews and commentaries in antimicrobial and anticancer chemotherapy 13: 151-171.

Ramos-Aires J, Plesiat P, Kocjancic-Curty L \& Kohler T (2004) Selection of an antibiotichypersusceptible mutant of Pseudomonas aeruginosa: identification of the GlmR transcriptional regulator. Antimicrobial agents and chemotherapy 48: 843-851.

Ramphal R, McNiece MT \& Polack FM (1981) Adherence of Pseudomonas aeruginosa to the injured cornea: a step in the pathogenesis of corneal infections. Annals of ophthalmology 13: 421-425.

Ramsey DM \& Wozniak DJ (2005) Understanding the control of Pseudomonas aeruginosa alginate synthesis and the prospects for management of chronic infections in cystic fibrosis. Molecular microbiology 56: 309-322.

Rangel SM, Diaz MH, Knoten CA, Zhang A \& Hauser AR (2015) The role of ExoS in dissemination of Pseudomonas aeruginosa during pneumonia. PLOS pathogens 11: e1004945.

Rashid MH \& Kornberg A (2000) Inorganic polyphosphate is needed for swimming, swarming, and twitching motilities of Pseudomonas aeruginosa. Proceedings of the National Academy of Sciences of the United States of America 97: 4885-4890.

Rayner RJ, Hiller EJ, Ispahani P \& Baker M (1990) Haemophilus infection in cystic fibrosis. Archives of disease in childhood 65: 255-258.

Read RC, Roberts P, Munro N, et al. (1992) Effect of Pseudomonas aeruginosa rhamnolipids on mucociliary transport and ciliary beating. Journal of applied physiology 72: 2271-2277.

Reimmann C, Beyeler M, Latifi A, Winteler H, Foglino M, Lazdunski A \& Haas D (1997) The global activator GacA of Pseudomonas aeruginosa PAO positively controls the production of the autoinducer N-butyryl-homoserine lactone and the formation of the virulence factors pyocyanin, cyanide, and lipase. Molecular microbiology 24: 309-319.

Reith J \& Mayer C (2011) Peptidoglycan turnover and recycling in Gram-positive bacteria. Applied microbiology and biotechnology 92: 1-11.

Remminghorst U \& Rehm BH (2006) Bacterial alginates: from biosynthesis to applications. Biotechnology letters 28: 1701-1712.

Reyes EA, Bale MJ, Cannon WH \& Matsen JM (1981) Identification of Pseudomonas aeruginosa by pyocyanin production on Tech agar. Journal of clinical microbiology 13: 456-458.

Rice LB (2008) Federal funding for the study of antimicrobial resistance in nosocomial pathogens: no ESKAPE. The Journal of infectious diseases 197: 1079-1081.

Richmond MH \& Sykes RB (1973) The beta-lactamases of Gram-negative bacteria and their possible physiological role. Advances in microbial physiology 9: 31-88.

Rietsch A, Vallet-Gely I, Dove SL \& Mekalanos JJ (2005) ExsE, a secreted regulator of type III secretion genes in Pseudomonas aeruginosa. Proceedings of the National Academy of Sciences of the United States of America 102: 8006-8011. 
Rivera I, Molina R, Lee M, Mobashery S \& Hermoso JA (2016) Orthologous and paralogous AmpD peptidoglycan amidases from Gram-negative bacteria. Microbial drug resistance.

Robicsek A, Jacoby GA \& Hooper DC (2006) The worldwide emergence of plasmidmediated quinolone resistance. The Lancet. Infectious diseases 6: 629-640.

Romeis T \& Holtje JV (1994) Penicillin-binding protein 7/8 of Escherichia coli is a DDendopeptidase. European journal of biochemistry / FEBS 224: 597-604.

Romeis T, Vollmer W \& Holtje JV (1993) Characterization of three different lytic transglycosylases in Escherichia coli. FEMS microbiology letters 111: 141-146.

Ropy A, Cabot G, Sanchez-Diener I, Aguilera C, Moya B, Ayala JA \& Oliver A (2015) Role of Pseudomonas aeruginosa low-molecular-mass penicillin-binding proteins in AmpC expression, beta-lactam resistance, and peptidoglycan structure. Antimicrobial agents and chemotherapy 59: 3925-3934.

Rose HD (1968) Chronic Pseudomonas aeruginosa pneumonia in a normal adult. Report of a case. Diseases of the chest 53: 643-648.

Ruiz N (2008) Bioinformatics identification of MurJ (MviN) as the peptidoglycan lipid II flippase in Escherichia coli. Proceedings of the National Academy of Sciences of the United States of America 105: 15553-15557.

Ruiz N (2015) Lipid flippases for bacterial peptidoglycan biosynthesis. Lipid insights 8: 21-31.

Ryder C, Byrd M \& Wozniak DJ (2007) Role of polysaccharides in Pseudomonas aeruginosa biofilm development. Current opinion in microbiology 10: 644-648.

Sacha P, Wieczorek P, Hauschild T, Zorawski M, Olszanska D \& Tryniszewska E (2008) Metallo-beta-lactamases of Pseudomonas aeruginosa--a novel mechanism resistance to beta-lactam antibiotics. Folia histochemica et cytobiologica 46: 137-142.

Sadikot RT, Blackwell TS, Christman JW \& Prince AS (2005) Pathogen-host interactions in Pseudomonas aeruginosa pneumonia. American journal of respiratory and critical care medicine 171: 1209-1223.

Saliba AM, Nascimento DO, Silva MC, et al. (2005) Eicosanoid-mediated proinflammatory activity of Pseudomonas aeruginosa ExoU. Cellular microbiology 7: 1811-1822.

Salton MR (1953) Studies of the bacterial cell wall. IV. The composition of the cell walls of some Gram-positive and Gram-negative bacteria. Biochimica et biophysica acta 10: 512-523.

Samonis G, Maraki S, Karageorgopoulos DE, Vouloumanou EK \& Falagas ME (2012) Synergy of fosfomycin with carbapenems, colistin, netilmicin, and tigecycline against multidrug-resistant Klebsiella pneumoniae, Escherichia coli, and Pseudomonas aeruginosa clinical isolates. European journal of clinical microbiology \& infectious diseases : official publication of the European Society of Clinical Microbiology 31: 695701.

Santos JM, Lobo M, Matos AP, De Pedro MA \& Arraiano CM (2002) The gene bolA regulates dacA (PBP5), dacC (PBP6) and ampC (AmpC), promoting normal morphology in Escherichia coli. Molecular microbiology 45: 1729-1740. 
Sarkar SK, Chowdhury C \& Ghosh AS (2010) Deletion of penicillin-binding protein 5 (PBP5) sensitises Escherichia coli cells to beta-lactam agents. International journal of antimicrobial agents 35: 244-249.

Sarkar SK, Dutta M, Chowdhury C, Kumar A \& Ghosh AS (2011) PBP5, PBP6 and DacD play different roles in intrinsic beta-lactam resistance of Escherichia coli. Microbiology 157: 2702-2707.

Sauvage E, Kerff F, Terrak M, Ayala JA \& Charlier P (2008) The penicillin-binding proteins: structure and role in peptidoglycan biosynthesis. FEMS microbiology reviews 32: 234-258.

Sawa T, Ohara M, Kurahashi K, et al. (1998) In vitro cellular toxicity predicts Pseudomonas aeruginosa virulence in lung infections. Infection and immunity 66: 32423249.

Schad PA \& Iglewski BH (1988) Nucleotide sequence and expression in Escherichia coli of the Pseudomonas aeruginosa lasA gene. Journal of bacteriology 170: 2784-2789.

Scheurwater E, Reid CW \& Clarke AJ (2008) Lytic transglycosylases: bacterial spacemaking autolysins. The international journal of biochemistry \& cell biology 40: 586-591.

Scheurwater EM, Pfeffer JM \& Clarke AJ (2007) Production and purification of the bacterial autolysin $\mathrm{N}$-acetylmuramoyl-L-alanine amidase $\mathrm{B}$ from Pseudomonas aeruginosa. Protein expression and purification 56: 128-137.

Schiffer G \& Holtje JV (1999) Cloning and characterization of PBP 1C, a third member of the multimodular class A penicillin-binding proteins of Escherichia coli. The Journal of biological chemistry 274: 32031-32039.

Schlaman HR, Spaink HP, Okker RJ \& Lugtenberg BJ (1989) Subcellular localization of the nodD gene product in Rhizobium leguminosarum. Journal of bacteriology 171: 46864693.

Schleifer KH \& Kandler O (1972) Peptidoglycan types of bacterial cell walls and their taxonomic implications. Bacteriological reviews 36: 407-477.

Schmidt DM, Hubbard BK \& Gerlt JA (2001) Evolution of enzymatic activities in the enolase superfamily: functional assignment of unknown proteins in Bacillus subtilis and Escherichia coli as L-Ala-D/L-Glu epimerases. Biochemistry 40: 15707-15715.

Schmidtke AJ \& Hanson ND (2008) Role of $a m p D$ homologs in overproduction of AmpC in clinical isolates of Pseudomonas aeruginosa. Antimicrobial agents and chemotherapy 52: 3922-3927.

Schneper L. MN, Mathee K. (2012) Anti-quorum sensing, anti-bacterial and immunomodulatory properties of Panax ginseng. ed.^eds.), p.^pp. Global Science Books.

Schroeder U, Henrich B, Fink J \& Plapp R (1994) Peptidase D of Escherichia coli K-12, a metallopeptidase of low substrate specificity. FEMS microbiology letters 123: 153-159.

Schweizer HP (2003) Efflux as a mechanism of resistance to antimicrobials in Pseudomonas aeruginosa and related bacteria: unanswered questions. Genetics and molecular research : GMR 2: 48-62. 
Secher I, Hermes I, Pre S, Carreau F \& Bahuet F (2005) [Surgical wound infections due to Pseudomonas aeruginosa in orthopedic surgery]. Medecine et maladies infectieuses 35: 149-154.

Seoane A, Francia MV \& Garcia Lobo JM (1992) Nucleotide sequence of the ampCampR region from the chromosome of Yersinia enterocolitica. Antimicrobial agents and chemotherapy 36: 1049-1052.

Seyfried PL \& Fraser DJ (1978) Pseudomonas aeruginosa in swimming pools related to the incidence of otitis externa infection. Health laboratory science 15: 50-57.

Shah IM, Laaberki MH, Popham DL \& Dworkin J (2008) A eukaryotic-like Ser/Thr kinase signals bacteria to exit dormancy in response to peptidoglycan fragments. Cell 135: 486496.

Sham LT, Butler EK, Lebar MD, Kahne D, Bernhardt TG \& Ruiz N (2014) Bacterial cell wall. MurJ is the flippase of lipid-linked precursors for peptidoglycan biogenesis. Science 345: 220-222.

Shanks RM, Caiazza NC, Hinsa SM, Toutain CM \& O'Toole GA (2006) Saccharomyces cerevisiae-based molecular tool kit for manipulation of genes from Gram-negative bacteria. Appl Environ Microbiol 72: 5027-5036.

Shaw KJ, Rather PN, Hare RS \& Miller GH (1993) Molecular genetics of aminoglycoside resistance genes and familial relationships of the aminoglycoside-modifying enzymes. Microbiological reviews 57: 138-163.

Shigemura K, Arakawa S, Sakai Y, Kinoshita S, Tanaka K \& Fujisawa M (2006) Complicated urinary tract infection caused by Pseudomonas aeruginosa in a single institution (1999-2003). International journal of urology : official journal of the Japanese Urological Association 13: 538-542.

Shimkets LJ \& Kaiser D (1982) Murein components rescue developmental sporulation of Myxococcus xanthus. Journal of bacteriology 152: 462-470.

Silby MW, Winstanley C, Godfrey SA, Levy SB \& Jackson RW (2011) Pseudomonas genomes: diverse and adaptable. FEMS microbiology reviews 35: 652-680.

Silver LL (2011) Challenges of antibacterial discovery. Clinical microbiology reviews 24: 71-109.

Smith JD, Kumarasiri M, Zhang W, et al. (2013) Structural analysis of the role of Pseudomonas aeruginosa penicillin-binding protein 5 in beta-lactam resistance. Antimicrobial agents and chemotherapy 57: 3137-3146.

Smith RS \& Iglewski BH (2003) P. aeruginosa quorum-sensing systems and virulence. Current opinion in microbiology 6: 56-60.

Solano C, Echeverz M \& Lasa I (2014) Biofilm dispersion and quorum sensing. Current opinion in microbiology 18: 96-104.

Solomons CC, Cotton EK, Dubois R \& Pinney M (1971) The use of buffered L-arginine in the treatment of cystic fibrosis. Pediatrics 47: 384-390.

Song J, Xie G, Elf PK, Young KD \& Jensen RA (1998) Comparative analysis of Pseudomonas aeruginosa penicillin-binding protein 7 in the context of its membership in the family of low-molecular-mass PBPs. Microbiology 144 ( Pt 4): 975-983. 
Song Z, Kong KF, Wu H, et al. (2010) Panax ginseng has anti-infective activity against opportunistic pathogen Pseudomonas aeruginosa by inhibiting quorum sensing, a bacterial communication process critical for establishing infection. Phytomedicine : international journal of phytotherapy and phytopharmacology 17: 1040-1046.

Spratt BG (1977) Properties of the penicillin-binding proteins of Escherichia coli K12. European journal of biochemistry 72: 341-352.

Spratt BG \& Strominger JL (1976) Identification of the major penicillin-binding proteins of Escherichia coli as D-alanine carboxypeptidase IA. Journal of bacteriology 127: 660-663.

Srikumar R, Paul CJ \& Poole K (2000) Influence of mutations in the mexR repressor gene on expression of the MexA-MexB-oprM multidrug efflux system of Pseudomonas aeruginosa. Journal of bacteriology 182: 1410-1414.

Srikumar R, Kon T, Gotoh N \& Poole K (1998) Expression of Pseudomonas aeruginosa multidrug efflux pumps MexA-MexB-OprM and MexC-MexD-OprJ in a multidrugsensitive Escherichia coli strain. Antimicrobial agents and chemotherapy 42: 65-71.

Stapper AP, Narasimhan G, Ohman DE, et al. (2004) Alginate production affects Pseudomonas aeruginosa biofilm development and architecture, but is not essential for biofilm formation. Journal of medical microbiology 53: 679-690.

Stevens TC, Ochoa CD, Morrow KA, et al. (2014) The Pseudomonas aeruginosa exoenzyme $\mathrm{Y}$ impairs endothelial cell proliferation and vascular repair following lung injury. American journal of physiology. Lung cellular and molecular physiology 306: L915-924.

Stewart PS (2002) Mechanisms of antibiotic resistance in bacterial biofilms. International journal of medical microbiology : IJMM 292: 107-113.

Stintzi A, Evans K, Meyer JM \& Poole K (1998) Quorum-sensing and siderophore biosynthesis in Pseudomonas aeruginosa: lasR/las/ mutants exhibit reduced pyoverdine biosynthesis. FEMS microbiology letters 166: 341-345.

Stover CK, Pham XQ, Erwin AL, et al. (2000) Complete genome sequence of Pseudomonas aeruginosa PAO1, an opportunistic pathogen. Nature 406: 959-964.

Strominger JL, Izaki K, Matsuhashi M \& Tipper DJ (1967) Peptidoglycan transpeptidase and D-alanine carboxypeptidase: penicillin-sensitive enzymatic reactions. Federation proceedings 26: 9-22.

Stubbs KA, Balcewich M, Mark BL \& Vocadlo DJ (2007) Small molecule inhibitors of a glycoside hydrolase attenuate inducible AmpC-mediated beta-lactam resistance. The Journal of biological chemistry 282: 21382-21391.

Stubbs KA, Scaffidi A, Debowski AW, Mark BL, Stick RV \& Vocadlo DJ (2008) Synthesis and use of mechanism-based protein-profiling probes for retaining beta-Dglucosaminidases facilitate identification of Pseudomonas aeruginosa NagZ. Journal of the American Chemical Society 130: 327-335.

Sugawara E, Nestorovich EM, Bezrukov SM \& Nikaido H (2006) Pseudomonas aeruginosa porin OprF exists in two different conformations. The Journal of biological chemistry 281: 16220-16229. 
Suzuki H, van Heijenoort Y, Tamura T, Mizoguchi J, Hirota Y \& van Heijenoort J (1980) In vitro peptidoglycan polymerization catalysed by penicillin binding protein $1 \mathrm{~b}$ of Escherichia coli K-12. FEBS letters 110: 245-249.

Sykes RB \& Matthew M (1976) The beta-lactamases of Gram-negative bacteria and their role in resistance to beta-lactam antibiotics. The Journal of antimicrobial chemotherapy 2: 115-157.

Tacconelli E, Smith G, Hieke K, Lafuma A \& Bastide P (2009) Epidemiology, medical outcomes and costs of catheter-related bloodstream infections in intensive care units of four European countries: literature- and registry-based estimates. The Journal of hospital infection 72: 97-103.

Takeuchi O \& Akira S (2010) Pattern recognition receptors and inflammation. Cell 140: 805-820.

Talsma SS (2007) Biofilms on medical devices. Home healthcare nurse 25: 589-594.

Tamura T, Imae Y \& Strominger JL (1976) Purification to homogeneity and properties of two D-alanine carboxypeptidases I from Escherichia coli. The Journal of biological chemistry 251: 414-423.

Tamura T, Suzuki H, Nishimura Y, Mizoguchi J \& Hirota Y (1980) On the process of cellular division in Escherichia coli: isolation and characterization of penicillin-binding proteins 1a, 1b, and 3. Proceedings of the National Academy of Sciences of the United States of America 77: 4499-4503.

Tan MW, Mahajan-Miklos S \& Ausubel FM (1999) Killing of Caenorhabditis elegans by Pseudomonas aeruginosa used to model mammalian bacterial pathogenesis. Proceedings of the National Academy of Sciences of the United States of America 96: 715-720.

Tangden T (2014) Combination antibiotic therapy for multidrug-resistant Gram-negative bacteria. Upsala journal of medical sciences 119: 149-153.

Tavares IM, Jolly L, Pompeo F, Leitao JH, Fialho AM, Sa-Correia I \& Mengin-Lecreulx D (2000) Identification of the Pseudomonas aeruginosa g/mM gene, encoding phosphoglucosamine mutase. Journal of bacteriology 182: 4453-4457.

Teixeira B, Rodulfo H, Carreno N, Guzman M, Salazar E \& De Donato M (2016) Aminoglycoside resistance genes in Pseudomonas aeruginosa isolates from Cumana, Venezuela. Revista do Instituto de Medicina Tropical de Sao Paulo 58: 13.

Templin MF, Ursinus A \& Holtje JV (1999) A defect in cell wall recycling triggers autolysis during the stationary growth phase of Escherichia coli. The EMBO journal 18: 4108-4117.

Terrak M, Ghosh TK, van Heijenoort J, et al. (1999) The catalytic, glycosyl transferase and acyl transferase modules of the cell wall peptidoglycan-polymerizing penicillinbinding protein 1b of Escherichia coli. Molecular microbiology 34: 350-364.

Tipper DJ \& Strominger JL (1965) Mechanism of action of penicillins: a proposal based on their structural similarity to acyl-D-alanyl-D-alanine. Proceedings of the National Academy of Sciences of the United States of America 54: 1133-1141.

Toleman MA, Simm AM, Murphy TA, Gales AC, Biedenbach DJ, Jones RN \& Walsh TR (2002) Molecular characterization of SPM-1, a novel metallo-beta-lactamase isolated in 
Latin America: report from the SENTRY antimicrobial surveillance programme. The Journal of antimicrobial chemotherapy 50: 673-679.

Tolg M, Schmidt H, Schierl R, Datz M \& Martin HH (1993) Dependence of induction of enterobacterial AmpC beta-lactamase on cell-wall peptidoglycan, as demonstrated in Proteus mirabilis and its wall-less protoplast L-form. Journal of general microbiology 139: 2715-2722.

Tomioka S, Nikaido T, Miyakawa T \& Matsuhashi M (1983) Mutation of the Nacetylmuramyl-L-alanine amidase gene of Escherichia coli K-12. Journal of bacteriology 156: 463-465.

Tong BC \& Barbul A (2004) Cellular and physiological effects of arginine. Mini reviews in medicinal chemistry 4: 823-832.

Torrens G, Perez-Gallego M, Moya B, et al. (2017) Targeting the permeability barrier and peptidoglycan recycling pathways to disarm Pseudomonas aeruginosa against the innate immune system. PloS one 12: e0181932.

Toutain CM, Zegans ME \& O'Toole GA (2005) Evidence for two flagellar stators and their role in the motility of Pseudomonas aeruginosa. Journal of bacteriology 187: 771777.

Tran JH \& Jacoby GA (2002) Mechanism of plasmid-mediated quinolone resistance. Proceedings of the National Academy of Sciences of the United States of America 99: 5638-5642.

Trias J \& Nikaido H (1990) Protein D2 channel of the Pseudomonas aeruginosa outer membrane has a binding site for basic amino acids and peptides. The Journal of biological chemistry 265: 15680-15684.

Tsui HC, Zhao G, Feng G, Leung HC \& Winkler ME (1994) The mutL repair gene of Escherichia coli K-12 forms a superoperon with a gene encoding a new cell-wall amidase. Molecular microbiology 11: 189-202.

Tummler B, Bosshammer J, Breitenstein S, et al. (1997) Infections with Pseudomonas aeruginosa in patients with cystic fibrosis. Behring Institute Mitteilungen 249-255.

Tuomanen E, Lindquist S, Sande S, Galleni M, Light K, Gage D \& Normark S (1991) Coordinate regulation of beta-lactamase induction and peptidoglycan composition by the amp operon. Science 251: 201-204.

Typas A, Banzhaf M, Gross CA \& Vollmer W (2011) From the regulation of peptidoglycan synthesis to bacterial growth and morphology. Nature reviews. Microbiology 10: 123-136.

Tzouvelekis LS \& Bonomo RA (1999) SHV-type beta-lactamases. Current pharmaceutical design 5: 847-864.

Uehara T \& Park JT (2003) Identification of MpaA, an amidase in Escherichia coli that hydrolyzes the gamma-D-glutamyl-meso-diaminopimelate bond in murein peptides. Journal of bacteriology 185: 679-682.

Uehara T \& Park JT (2004) The N-acetyl-D-glucosamine kinase of Escherichia coli and its role in murein recycling. Journal of bacteriology 186: 7273-7279. 
Uehara T \& Park JT (2007) An anhydro-N-acetylmuramyl-L-alanine amidase with broad specificity tethered to the outer membrane of Escherichia coli. Journal of bacteriology 189: $5634-5641$.

Uehara T, Dinh T \& Bernhardt TG (2009) LytM-domain factors are required for daughter cell separation and rapid ampicillin-induced lysis in Escherichia coli. Journal of bacteriology 191: 5094-5107.

Uehara T, Parzych KR, Dinh T \& Bernhardt TG (2010) Daughter cell separation is controlled by cytokinetic ring-activated cell wall hydrolysis. The EMBO journal 29: 14121422.

Uehara T, Suefuji K, Jaeger T, Mayer C \& Park JT (2006) MurQ etherase is required by Escherichia coli in order to metabolize anhydro-N-acetylmuramic acid obtained either from the environment or from its own cell wall. Journal of bacteriology 188: 1660-1662.

Uehara T, Suefuji K, Valbuena N, Meehan B, Donegan M \& Park JT (2005) Recycling of the anhydro-N-acetylmuramic acid derived from cell wall murein involves a two-step conversion to N-acetylglucosamine-phosphate. Journal of bacteriology 187: 3643-3649.

Ueno T, Oosawa K \& Aizawa S (1992) M ring, S ring and proximal rod of the flagellar basal body of Salmonella typhimurium are composed of subunits of a single protein, FliF. Journal of molecular biology 227: 672-677.

Uwate M, Ichise YK, Shirai A, Omasa T, Nakae T \& Maseda H (2013) Two routes of MexS-MexT-mediated regulation of MexEF-OprN and MexAB-OprM efflux pump expression in Pseudomonas aeruginosa. Microbiology and immunology 57: 263-272.

Vadlamani G, Thomas MD, Patel TR, et al. (2015) The beta-lactamase gene regulator AmpR is a tetramer that recognizes and binds the D-Ala-D-Ala motif of its repressor UDP-N-acetylmuramic acid (MurNAc)-pentapeptide. The Journal of biological chemistry 290: 2630-2643.

Valderrey AD, Pozuelo MJ, Jimenez PA, Macia MD, Oliver A \& Rotger R (2010) Chronic colonization by Pseudomonas aeruginosa of patients with obstructive lung diseases: cystic fibrosis, bronchiectasis, and chronic obstructive pulmonary disease. Diagnostic microbiology and infectious disease 68: 20-27.

Vallis AJ, Yahr TL, Barbieri JT \& Frank DW (1999) Regulation of ExoS production and secretion by Pseudomonas aeruginosa in response to tissue culture conditions. Infection and immunity 67: 914-920.

van der Linden MP, de Haan L, Hoyer MA \& Keck W (1992) Possible role of Escherichia coli penicillin-binding protein 6 in stabilization of stationary-phase peptidoglycan. Journal of bacteriology 174: 7572-7578.

van Heijenoort J (2007) Lipid intermediates in the biosynthesis of bacterial peptidoglycan. Microbiology and molecular biology reviews : MMBR 71: 620-635.

van Heijenoort J (2011) Peptidoglycan hydrolases of Escherichia coli. Microbiology and molecular biology reviews : MMBR 75: 636-663.

van Heijenoort J, Parquet C, Flouret B \& van Heijenoort Y (1975) Envelope-bound Nacetylmuramyl-L-alanine amidase of Escherichia coli K 12. Purification and properties of the enzyme. European journal of biochemistry 58: 611-619. 
Van Heijenoort Y \& Van Heijenoort J (1971) Study of the N-acetylmuramyl-L-alanine amidase activity in Escherichia coli. FEBS letters 15: 137-141.

Vasil ML (1986) Pseudomonas aeruginosa: biology, mechanisms of virulence, epidemiology. The Journal of pediatrics 108: 800-805.

Venter H, Mowla R, Ohene-Agyei T \& Ma S (2015) RND-type drug efflux pumps from Gram-negative bacteria: molecular mechanism and inhibition. Frontiers in microbiology 6: 377.

Vento S, Cainelli F \& Temesgen Z (2008) Lung infections after cancer chemotherapy. The Lancet. Oncology 9: 982-992.

Visca P, Imperi F \& Lamont IL (2007) Pyoverdine siderophores: from biogenesis to biosignificance. Trends in microbiology 15: 22-30.

Vogler AP, Homma M, Irikura VM \& Macnab RM (1991) Salmonella typhimurium mutants defective in flagellar filament regrowth and sequence similarity of Flil to F0F1, vacuolar, and archaebacterial ATPase subunits. Journal of bacteriology 173: 3564-3572.

Vollmer W \& Holtje JV (2004) The architecture of the murein (peptidoglycan) in Gramnegative bacteria: vertical scaffold or horizontal layer(s)? Journal of bacteriology 186: 5978-5987.

Vollmer W, Blanot D \& de Pedro MA (2008) Peptidoglycan structure and architecture. FEMS microbiology reviews 32: 149-167.

Vollmer W, Joris B, Charlier P \& Foster S (2008) Bacterial peptidoglycan (murein) hydrolases. FEMS microbiology reviews 32: 259-286.

Votsch W \& Templin MF (2000) Characterization of a beta-N-acetylglucosaminidase of Escherichia coli and elucidation of its role in muropeptide recycling and beta-lactamase induction. The Journal of biological chemistry 275: 39032-39038.

Walker JM (1994) The bicinchoninic acid (BCA) assay for protein quantitation. Methods in molecular biology 32: 5-8.

Walther-Rasmussen J \& Hoiby N (2002) Plasmid-borne AmpC beta-lactamases. Canadian journal of microbiology 48: 479-493.

Watanabe M, lyobe S, Inoue M \& Mitsuhashi S (1991) Transferable imipenem resistance in Pseudomonas aeruginosa. Antimicrobial agents and chemotherapy 35: 147-151.

Waters CM \& Bassler BL (2005) Quorum sensing: cell-to-cell communication in bacteria. Annu. Rev. Cell Dev. Biol. 21: 319-346.

Waters V \& Smyth A (2015) Cystic fibrosis microbiology: Advances in antimicrobial therapy. Journal of cystic fibrosis : official journal of the European Cystic Fibrosis Society 14: 551-560.

Watt SR \& Clarke AJ (1994) Initial characterization of two extracellular autolysins from Pseudomonas aeruginosa PAO1. Journal of bacteriology 176: 4784-4789.

Weidel W \& Pelzer H (1964) Bagshaped macromolecules--a new outlook on bacterial cell walls. Advances in enzymology and related subjects of biochemistry 26: 193-232. 
Weldhagen GF, Poirel L \& Nordmann P (2003) Ambler class A extended-spectrum betalactamases in Pseudomonas aeruginosa: novel developments and clinical impact. Antimicrobial agents and chemotherapy 47: 2385-2392.

Werner V, Sanders CC, Sanders WE, Jr. \& Goering RV (1985) Role of beta-lactamases and outer membrane proteins in multiple beta-lactam resistance of Enterobacter cloacae. Antimicrobial agents and chemotherapy 27: 455-459.

West SE, Zeng L, Lee BL, et al. (2002) Respiratory infections with Pseudomonas aeruginosa in children with cystic fibrosis: early detection by serology and assessment of risk factors. JAMA 287: 2958-2967.

Westfall LW, Carty NL, Layland N, Kuan P, Colmer-Hamood JA \& Hamood AN (2006) mvaT mutation modifies the expression of the Pseudomonas aeruginosa multidrug efflux operon mexEF-oprN. FEMS microbiology letters 255: 247-254.

Whatmore AM \& Reed RH (1990) Determination of turgor pressure in Bacillus subtilis: a possible role for $\mathrm{K}+$ in turgor regulation. Journal of general microbiology 136: 2521-2526.

White RJ \& Pasternak CA (1967) The purification and properties of N-acetylglucosamine 6-phosphate deacetylase from Escherichia coli. The Biochemical journal 105: 121-125.

Whiteley M, Lee KM \& Greenberg EP (1999) Identification of genes controlled by quorum sensing in Pseudomonas aeruginosa. Proceedings of the National Academy of Sciences of the United States of America 96: 13904-13909.

Wiedemann B, Pfeifle D, Wiegand I \& Janas E (1998) Beta-lactamase induction and cell wall recycling in Gram-negative bacteria. Drug resistance updates : reviews and commentaries in antimicrobial and anticancer chemotherapy 1: 223-226.

Winsor GL, Griffiths EJ, Lo R, Dhillon BK, Shay JA \& Brinkman FS (2016) Enhanced annotations and features for comparing thousands of Pseudomonas genomes in the Pseudomonas genome database. Nucleic acids research 44: D646-653.

Winstanley C, O'Brien S \& Brockhurst MA (2016) Pseudomonas aeruginosa evolutionary adaptation and diversification in cystic fibrosis chronic lung infections. Trends in microbiology 24: 327-337.

Wise EM, Jr. \& Park JT (1965) Penicillin: its basic site of action as an inhibitor of a peptide cross-linking reaction in cell wall mucopeptide synthesis. Proceedings of the National Academy of Sciences of the United States of America 54: 75-81.

Wolf AJ \& Underhill DM (2018) Peptidoglycan recognition by the innate immune system. Nature reviews. Immunology 18: 243-254.

Wolfert MA, Roychowdhury A \& Boons GJ (2007) Modification of the structure of peptidoglycan is a strategy to avoid detection by nucleotide-binding oligomerization domain protein 1. Infection and immunity 75: 706-713.

Wolfert MA, Murray TF, Boons GJ \& Moore JN (2002) The origin of the synergistic effect of muramyl dipeptide with endotoxin and peptidoglycan. The Journal of biological chemistry 277: 39179-39186.

Woodhams KL, Chan JM, Lenz JD, Hackett KT \& Dillard JP (2013) Peptidoglycan fragment release from Neisseria meningitidis. Infection and immunity 81: 3490-3498. 
World Health Organization (2017) WHO publishes list of bacteria for which new antibiotics are urgently needed.

Wu BY, Peng CT, Tsai CH \& Chiu HH (1999) Community-acquired Pseudomonas aeruginosa bacteremia and sepsis in previously healthy infants. Acta paediatrica Taiwanica = Taiwan er ke yi xue hui za zhi 40: 233-236.

Wu H, Lee B, Yang L, et al. (2011) Effects of ginseng on Pseudomonas aeruginosa motility and biofilm formation. FEMS immunology and medical microbiology 62: 49-56.

Wu H, Lee B, Yang L, et al. (2011) Effects of ginseng on Pseudomonas aeruginosa motility and biofilm formation. FEMS Immunology \& Medical Microbiology 62: 49-56.

Yahr TL \& Frank DW (1994) Transcriptional organization of the trans-regulatory locus which controls exoenzyme $S$ synthesis in Pseudomonas aeruginosa. Journal of bacteriology 176: 3832-3838.

Yahr TL \& Greenberg EP (2004) The genetic basis for the commitment to chronic versus acute infection in Pseudomonas aeruginosa. Molecular cell 16: 497-498.

Yakhnina AA, McManus HR \& Bernhardt TG (2015) The cell wall amidase AmiB is essential for Pseudomonas aeruginosa cell division, drug resistance and viability. Molecular microbiology 97: 957-973.

Yamagishi J, Yoshida H, Yamayoshi M \& Nakamura S (1986) Nalidixic acid-resistant mutations of the gyrB gene of Escherichia coli. Molecular \& general genetics : MGG 204: 367-373.

Yamagishi J, Furutani Y, Inoue S, Ohue T, Nakamura S \& Shimizu M (1981) New nalidixic acid resistance mutations related to deoxyribonucleic acid gyrase activity. Journal of bacteriology 148: 450-458.

Yamaguchi T, Blazquez B, Hesek D, et al. (2012) Inhibitors for bacterial cell-wall recycling. ACS medicinal chemistry letters 3: 238-242.

Yang TC, Chen TF, Tsai JJ \& Hu RM (2013) AmpG is required for BlaXc beta-lactamase expression in Xanthomonas campestris pv. campestris str. 17. FEMS microbiology letters 340: 101-108.

Yang Y \& Bush K (1995) Oxacillin hydrolysis by the LCR-1 beta-lactamase. Antimicrobial agents and chemotherapy 39: 1209.

Yem DW \& Wu HC (1976) Purification and properties of beta-N-acetylglucosaminidase from Escherichia coli. Journal of bacteriology 125: 324-331.

Yem DW \& Wu HC (1976) Isolation of Escherichia coli K-12 mutants with altered level of beta-N-acetylglucosaminidase. Journal of bacteriology 125: 372-373.

Yeo SG, Park DC, Hong SM, Cha Cl \& Kim MG (2007) Bacteriology of chronic suppurative otitis media--a multicenter study. Acta oto-laryngologica 127: 1062-1067.

Yildirim S, Nursal TZ, Tarim A, et al. (2005) Bacteriological profile and antibiotic resistance: comparison of findings in a burn intensive care unit, other intensive care units, and the hospital services unit of a single center. The Journal of burn care \& rehabilitation 26: 488-492.

Yoshimura F \& Nikaido H (1982) Permeability of Pseudomonas aeruginosa outer membrane to hydrophilic solutes. Journal of bacteriology 152: 636-642. 
Yoshimura F, Zalman LS \& Nikaido H (1983) Purification and properties of Pseudomonas aeruginosa porin. The Journal of biological chemistry 258: 2308-2314.

Yum HK, Park IN, Shin BM \& Choi SJ (2014) Recurrent Pseudomonas aeruginosa infection in chronic lung diseases: relapse or reinfection? Tuberculosis and respiratory diseases 77: 172-177.

Yunck R, Cho H \& Bernhardt TG (2016) Identification of MltG as a potential terminase for peptidoglycan polymerization in bacteria. Molecular microbiology 99: 700-718.

Zamorano L, Moya B, Juan C \& Oliver A (2010) Differential beta-lactam resistance response driven by $a m p D$ or $d a c B$ (PBP4) inactivation in genetically diverse Pseudomonas aeruginosa strains. The Journal of antimicrobial chemotherapy 65: 15401542.

Zamorano L, Moya B, Juan C, Mulet X, Blazquez J \& Oliver A (2014) The Pseudomonas aeruginosa CreBC two-component system plays a major role in the response to betalactams, fitness, biofilm growth, and global regulation. Antimicrobial agents and chemotherapy 58: 5084-5095.

Zamorano L, Reeve TM, Juan C, et al. (2011) AmpG inactivation restores susceptibility of pan-beta-lactam-resistant Pseudomonas aeruginosa clinical strains. Antimicrobial agents and chemotherapy 55: 1990-1996.

Zamorano L, Reeve TM, Deng L, et al. (2010) NagZ inactivation prevents and reverts beta-lactam resistance, driven by AmpD and PBP 4 mutations, in Pseudomonas aeruginosa. Antimicrobial agents and chemotherapy 54: 3557-3563.

Zawadzke LE, Bugg TD \& Walsh CT (1991) Existence of two D-alanine:D-alanine ligases in Escherichia coli: cloning and sequencing of the ddlA gene and purification and characterization of the DdIA and DdIB enzymes. Biochemistry 30: 1673-1682.

Zemelman R, Norambuena R, Vergara L \& Gacitua R (1987) Beta-lactam antibiotics: grouping according to their chemical structure and bacteriological properties. Revista medica de Chile 115: 983-991.

Zeng X \& Lin J (2013) Beta-lactamase induction and cell wall metabolism in Gramnegative bacteria. Frontiers in microbiology 4: 128.

Zhang JH, Chung TD \& Oldenburg KR (1999) A simple statistical parameter for use in evaluation and validation of high throughput screening assays. Journal of biomolecular screening 4: 67-73.

Zhang W, Lee M, Hesek D, Lastochkin E, Boggess B \& Mobashery S (2013) Reactions of the three AmpD enzymes of Pseudomonas aeruginosa. Journal of the American Chemical Society 135: 4950-4953.

Zhang W, Lee M, Hesek D, Lastochkin E, Boggess B \& Mobashery S (2013) Reactions of the three AmpD enzymes of Pseudomonas aeruginosa. Journal of the American Chemical Society.

Zhang Y, Bao Q, Gagnon LA, Huletsky A, Oliver A, Jin S \& Langaee T (2010) ampG gene of Pseudomonas aeruginosa and its role in beta-lactamase expression. Antimicrobial agents and chemotherapy 54: 4772-4779.

Zhao WH \& Hu ZQ (2010) Beta-lactamases identified in clinical isolates of Pseudomonas aeruginosa. Critical reviews in microbiology 36: 245-258. 
Zincke D, Balasubramanian D, Silver LL \& Mathee K (2015) Characterization of a carbapenem-hydrolyzing enzyme, PoxB, in Pseudomonas aeruginosa PAO1. Antimicrobial agents and chemotherapy 60: 936-945.

Zincke D, Balasubramanian D, Silver LL \& Mathee K (2016) Characterization of a carbapenem-hydrolyzing Enzyme, PoxB, in Pseudomonas aeruginosa PAO1. Antimicrobial agents and chemotherapy 60: 936-945. 
VITA

SUPURNA DHAR

$\begin{array}{ll}2004-2007 & \begin{array}{l}\text { Bachelor of Sciences (BSc.) } \\ \text { Osmania University } \\ \text { Hyderabad, India }\end{array} \\ 2007-2010 \quad \begin{array}{l}\text { Master of Sciences (MSc) } \\ \text { Manipal University } \\ \text { Karnataka, India }\end{array} \\ \text { PhD Candidate, } \\ \text { HWCOM, Florida International University } \\ \text { Miami, Florida }\end{array}$

\section{PUBLICATIONS AND PRESENTATIONS}

1. Lee $\mathrm{M}^{*}$, Dhar $\mathrm{S}^{*}$, De Benedetti S, Hesek D, Boggess B, Blazquez B, Mathee $\mathrm{K}$, Mobashery S. "Muropeptides in Pseudomonas aeruginosa and their Role as Elicitors of $\beta$-Lactam-Antibiotic Resistance." Angewandte Chemie International Edition 55.24 (2016): 6882-6886. Original Article ( ${ }^{*}$ Equal Contribution).

2. Pawitwar S, Dhar S, Tiwari S, Ojha CR, Lapierre J, Martins K, Rodzinski A, Parira T, Paudel I, Li JJ, Dutta RK, Silva MR, Kaushik A, El-Hage N. "Overview on the Current Status of Zika Virus Pathogenesis and Animal Related Research". J Neuroimmune Pharmacol (2017). Doi:10.1007/s11481-017-97438. Original Article.

3. Dhar S, Kumari H, Balasubramanian D, Mathee K. "Cell-wall recycling and synthesis in Escherichia coli and Pseudomonas aeruginosa- their role in development of antibiotic resistance." Journal of Medical Microbiology. Nov 29, 2017.

4. Dhar S, Lee M, Hesek D, Boggess W, Kumari H, Scheneper L, Mathee K, Mobashery S (2015). Identification of AmpR Effectors in Pseudomonas aeruginosa Regulating $\beta$-Lactam Resistance. 10th Cold Spring Harbor meeting on Microbial Pathogenesis and Host Response, Cold Spring Harbor, NY.

5. Dhar S, Kumari H, Mathee K (2018). Elucidating the role of FlgJ in Pseudomonas aeruginosa. ASM MICROBE 2018, Atlanta, GA.

6. Dhar S, Lee M, Beneditti De S, Hesek D, Boggess W, Kumari H, Schneper L, Mobashery S, Mathee K (2017). Characterization of muramyl peptides 
regulating $P$. aeruginosa AmpR. American Society of Microbiology ASM. 103rd Southeastern Branch Annual Meeting, St. Petersburg, FL.

7. Dhar S, Kumari $\mathrm{H}$, Mathee $\mathrm{K}$ (2018). Elucidating the role of PA1085 in Pseudomonas aeruginosa. FIU 20th Biomedical and Comparative Immunology Symposium (Awarded 1st place). 